\title{
Video-derived Observations of Longshore Currents
}

Anna Cohen 


\section{Preface}

The present M.Sc. thesis forms the completion of my studies at the Delft University of Technology, Faculty of Civil Engineering and Geosciences, Department of Civil Engineering, Divisions of Hydraulic Engineering and Fluid Mechanics. The study has been carried out at WL I Delft Hydraulics and was sponsored by the Dutch Ministry of Public Works Rijkswaterstaat (in the framework of the 'Voortschrijdend Onderzoeks Programma' VOP, contract RKZ1329) and the EU-funded CoastView Project under contract number EVK3-CT-2001-00054.

I would like to thank my supervisors, prof. dr. ir. M.J.F. Stive (Delft University of Technology), prof. dr. ir. J.A. Battjes (Delft University of Technology), dr. ir. P.J. Visser (Delft University of Technology) and ir. S.G.J. Aarninkhof (WL I Delft Hydraulics and Delft University of Technology) for their help and support during the realization of this thesis.

Furthermore I would like to thank Aart Kroon (Utrecht University) for his help and knowledge during the field experiments and for providing the needed equipment, Robin Morelissen (WL I Delft Hydraulics) for helping me to really 'get started with Matlab', Ab van Dongeren (WL I Delft Hydraulics) for his very useful lectures on spectral analysis and Chris Chickadel (Oregon State University) for taking the time to help me get familiar with time stack analysis.

Special thanks go out to my experimental helpers, Martijn du Prie, Nelleke Roeterink, Merijn Hillen and Linda Kusters, for their physical and mental support during rough and sunny weather. And of course I would like to thank the other graduate students at WL I Delft Hydraulics for creating an enjoyable atmosphere at our working space.

Anna Cohen 


\section{Contents}

\section{List of Figures}

\section{List of Tables}

\section{List of Symbols}

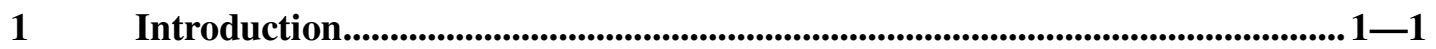

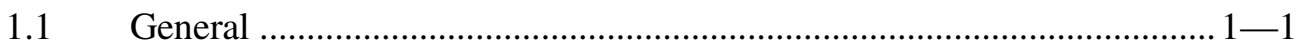

1.1.1 Introduction .......................................................................... 1 -

1.1.2 Longshore currents ......................................................... 1 - 1

1.1.3 Measuring longshore currents ................................................ $1-2$

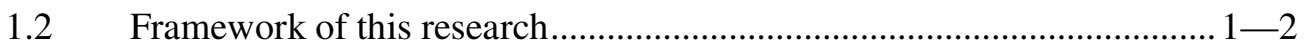

1.2.1 The CoastView project ............................................................. 1 -2

1.2.2 The Argus research program.............................................. 1-3

1.3 Research objectives and outline of this thesis ..................................... 1-8

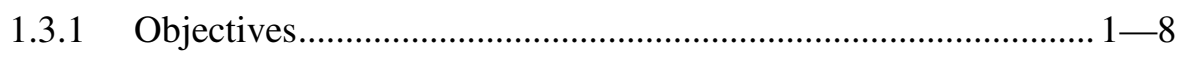

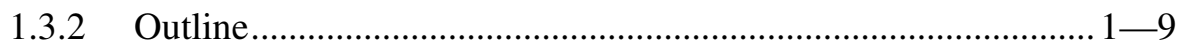

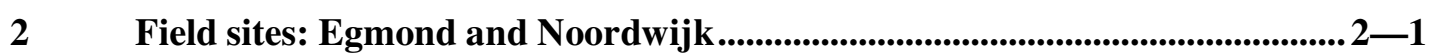

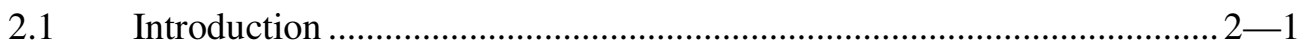

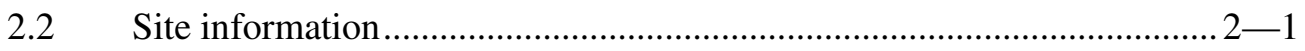

2.2.1 The Holland coast ............................................................... 2-1

2.2.2 Geomorphology …………............................................... 2-2

2.2.3 Hydrodynamic conditions............................................... 2-2

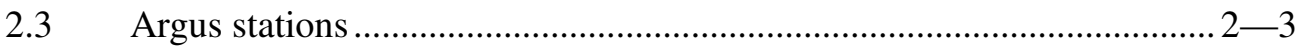

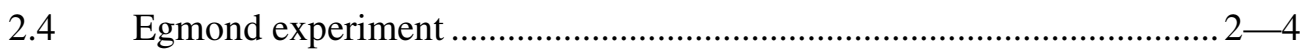

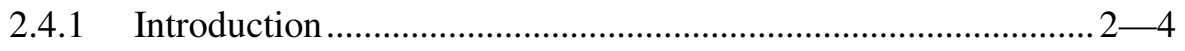




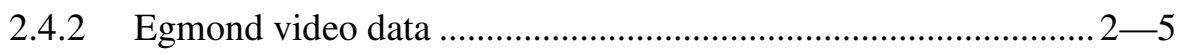

2.4.3 Egmond ground truth data .................................................... 2-7

2.4.4 Egmond data selection ......................................................... 2-7

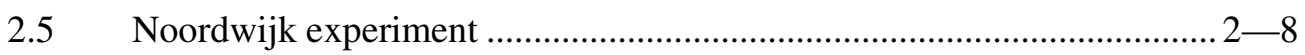

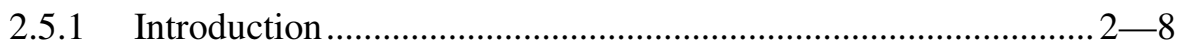

2.5.2 Noordwijk video data ...................................................... 2

2.5.3 Noordwijk ground truth data .................................................... 2-9

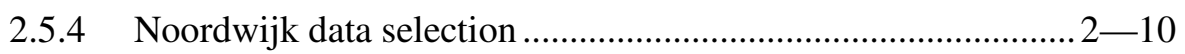

$3 \quad$ Model description ................................................................................................................... 3-1

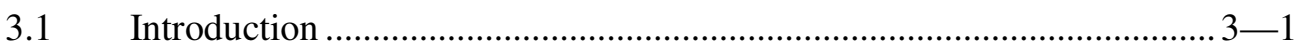

3.2 The Optical Current Meter (OCM) ..................................................... 3-1

3.3 Model parameters ............................................................... 3 - 6

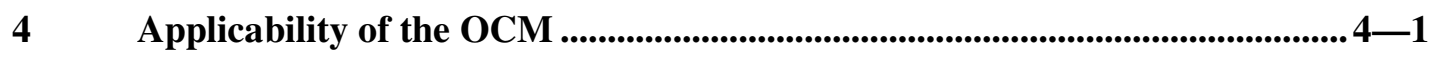

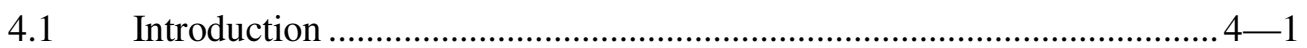

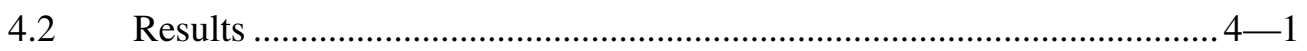

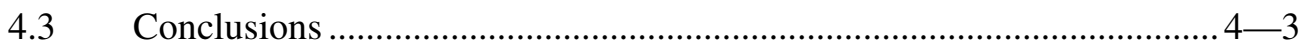

4.4 Discussion on the sampling spacing ................................................. $4-4$

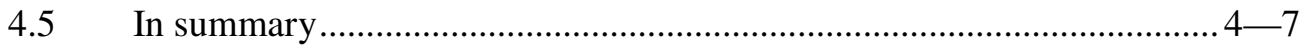

$5 \quad$ Accuracy of the OCM ............................................................................................5

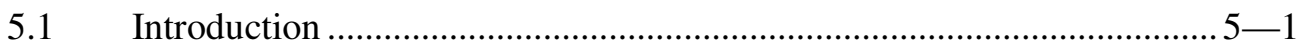

5.2 Calibration and validation of the model parameters .............................. 5-1

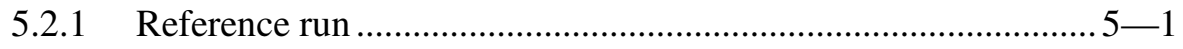

5.2.2 Time window and time shift ................................................... 5-2

5.2.3 Minimum wavenumber............................................................ 5 -4

5.2.4 Probability of the model fit.................................................. $5-8$

5.2.5 Confidence interval of the mean velocity ................................ 5-10 


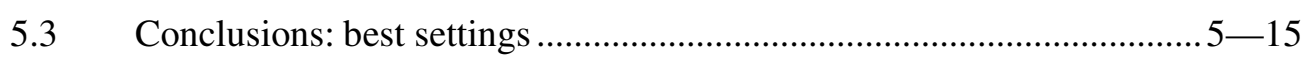

6 Discussion on the utility of the OCM ........................................................6-1

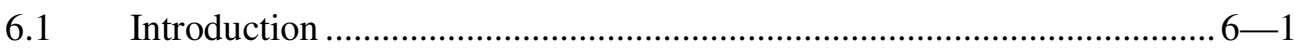

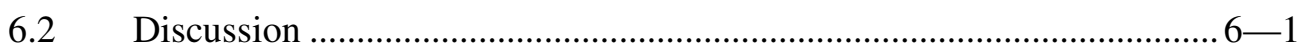

6.2.1 Utility regarding accuracy ………………............................ 6 -1

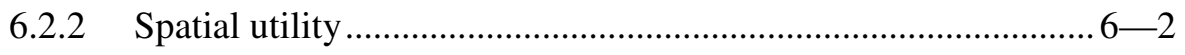

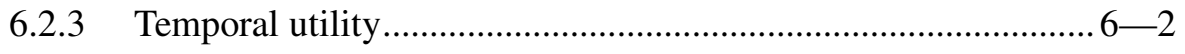

6.2.4 Meteorological utility ....................................................... 6-3

$7 \quad$ Conclusions and recommendations ................................................................................. 7-1

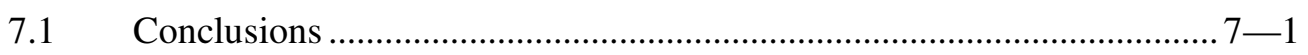

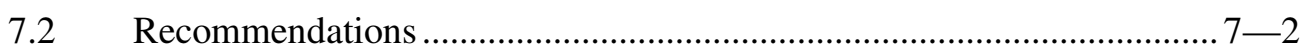

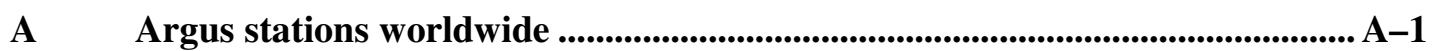

B Geo-referencing of oblique video data ............................................................... B-1

C Field measurement equipment .................................................................................. C-1

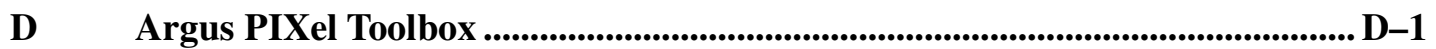

E Data selection Egmond and Noordwijk experiment........................................... E-1 


\section{List of Figures}

Figure 1-1 Longshore intensity time-stack, collected during SandyDuck (1997) ....................i

Figure 1-2 OCM model validation against EMF-measured velocities at Noordwijk ..............ii

Figure 1-3 Video-derived cross-shore distribution of alongshore flow velocities at Noordwijk. .ii

Figure 1-1 An example of a tripod stand alone measuring frame ................................... 1-2

Figure 1-2 Data transmission from the Argus camera to the internet............................... 1-4

Figure 1-3 Example of the Argus co-ordinate system (Noordwijk aan Zee) ..................... 1-5

Figure 1-4 Relation between image $(\mathrm{u}, \mathrm{v})$ and real world $(\mathrm{x}, \mathrm{y}, \mathrm{z})$ co-ordinates................... $1-6$

Figure 1-5 A snapshot image at Egmond aan Zee.......................................................... 1-6

Figure 1-6 A time exposure image at Egmond aan Zee .................................................... 1-6

Figure 1-7 A variance image at Egmond aan Zee ........................................................... 1-7

Figure 1-8 Relation between a pixel intensity time series and a wave height signal at Duck,

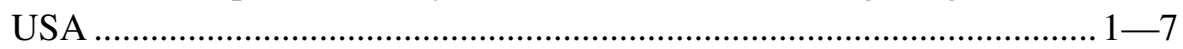

Figure 1-9 A time stack image collected along a cross-shore array at Duck, USA ........... 1-8

Figure 1-10 Snapshot of the surfzone at Duck NC. The line indicates the location of the alongshore pixel array where the video record was taken. ...................... $1-8$

Figure 1-11 A time stack, collected at Duck NC. The time stack reveals the bright horizontal bands of passing breaking waves and the oblique traces of foam patches drifting with the prevailing longshore current........................................ $1-8$

Figure 2-1 Map of Egmond field site ……………......................................................... 2-1

Figure 2-2 Map of Noordwijk field site ..................................................................... 2-1

Figure 2-3 The bar system in the nearshore zone along the Holland coast.......................2-2

Figure 2-4 Coast3D tower with Argus station in the top at the Egmond beach................2-4

Figure 2-5 Hotel "Huis Ter Duin" in Noordwijk aan Zee .............................................. 2-4

Figure 2-6 Argus station at the roof of hotel "Huis Ter Duin" .......................................... 2-4

Figure 2-7 Wave height during the Egmond measurements.......................................... 2-5

Figure 2-8 Wave period during the Egmond measurements.......................................... 2-5

Figure 2-9 Wave angle during the Egmond measurements .......................................... 2-5

Figure 2-10 Water level during the Egmond measurements........................................... 2-5

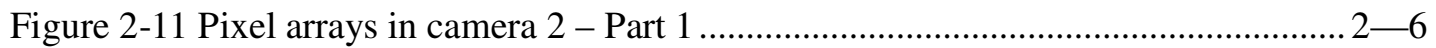

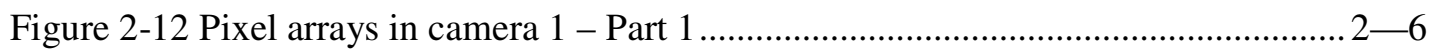

Figure 2-13 Pixel arrays in camera 2 - Part 2 2........................................................ 2-6

Figure 2-14 Pixel arrays in camera 1 - Part 2 2...................................................... 2-6

Figure 2-15 Pixel arrays in camera 2 - Part 3 ......................................................... 2-7 


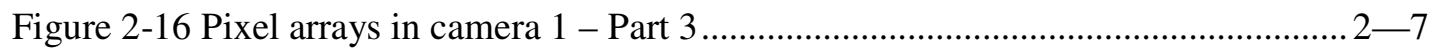

Figure 2-17 Wave height during the Noordwijk measurements .....................................2-8

Figure 2-18 Wave period during the Noordwijk measurements.......................................2 2-8

Figure 2-19 Wave angle during the Noordwijk measurements ...................................... 2-8

Figure 2-20 Water level during the Noordwijk measurements......................................... 2-8

Figure 2-21 Pixel arrays in view of camera 4, Noordwijk aan Zee..................................2-9

Figure 2-22 Pixel arrays in view of camera 5, Noordwijk aan Zee...................................2 2-9

Figure 3-2 A frequency-wavenumber spectrum..................................................... $3-2$

Figure 3-3 A velocity-wavenumber spectrum ...........................................................

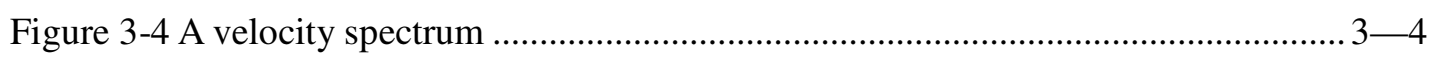

Figure 3-5 An observed velocity spectrum, together with the model of the velocity spectrum

Figure 4-1 OCM velocity estimates together with their standard deviation plotted against time for the Egmond data set.

Figure 4-2 OCM velocity estimates together with their standard deviation plotted against time for the Noordwijk data set

Figure 4-3 Mean $\mathrm{V}_{\text {video }}$ plotted against mean $\mathrm{V}_{\mathrm{gt}}$ for the Egmond data set ....................... 4-3

Figure 4-4 Mean $V_{\text {video }}$ plotted against mean $V_{\mathrm{gt}}$ for the Noordwijk data set.................... 4-3

Figure 4-5 A time stack image at Egmond showing the intensity bands.......................... 4-4

Figure 4-6 A time stack image at Noordwijk showing a smooth longshore intensity course

Figure 4-7 Mean $\mathrm{V}_{\text {video }}$ plotted against mean $\mathrm{V}_{\mathrm{gt}}$ using a sampling spacing $\Delta \mathrm{y}=0.10 \mathrm{~m}$ at Noordwijk $4-5$

Figure 4-8 Mean $\mathrm{V}_{\text {video }}$ plotted against mean $\mathrm{V}_{\mathrm{gt}}$ using a sampling spacing $\Delta \mathrm{y}=0.25 \mathrm{~m}$ at Noordwijk.

Figure 4-9 Mean $\mathrm{V}_{\text {video }}$ plotted against mean $\mathrm{V}_{\mathrm{gt}}$ using a sampling spacing $\Delta \mathrm{y}=0.50 \mathrm{~m}$ at Noordwijk. $4-5$

Figure 4-10 Mean $\mathrm{V}_{\text {video }}$ plotted against mean $\mathrm{V}_{\mathrm{gt}}$ using a sampling spacing $\Delta \mathrm{y}=0.75 \mathrm{~m}$ at Noordwijk. $4-5$

Figure 4-11 Mean $\mathrm{V}_{\text {video }}$ plotted against mean $\mathrm{V}_{\mathrm{gt}}$ using a sampling spacing $\Delta \mathrm{y}=1.00 \mathrm{~m}$ at Noordwijk.

Figure 4-12 Mean $\mathrm{V}_{\text {video }}$ plotted against mean $\mathrm{V}_{\mathrm{gt}}$ using a sampling spacing $\Delta \mathrm{y}=0.25 \mathrm{~m}$ at Egmond.

Figure 4-13 Mean $V_{\text {video }}$ plotted against mean $V_{\text {gt }}$ using a sampling spacing $\Delta y=2.00 \mathrm{~m}$ at Egmond. 4-6

Figure 5-1 Mean $\mathrm{V}_{\text {video }}$ plotted against mean $\mathrm{V}_{\mathrm{gt}}$ for the reference run ............................5-2

Figure 5-2 Mean $\mathrm{V}_{\text {video }}$ plotted against mean $\mathrm{V}_{\mathrm{gt}}$ with $\mathrm{T}_{\mathrm{w}}=8 \mathrm{~s}$ and $\mathrm{Ts}=4 \mathrm{~s} \ldots \ldots \ldots \ldots \ldots \ldots \ldots . .5-3$ 
Figure 5-3 Mean $\mathrm{V}_{\text {video }}$ plotted against mean $\mathrm{V}_{\mathrm{gt}}$ with $\mathrm{T}_{\mathrm{w}}=16 \mathrm{~s}$ and $\mathrm{Ts}=8 \mathrm{~s} \ldots \ldots \ldots \ldots \ldots \ldots . .5-3$

Figure 5-4 Mean $\mathrm{V}_{\text {video }}$ plotted against mean $\mathrm{V}_{\mathrm{gt}}$ with $\mathrm{T}_{\mathrm{w}}=32 \mathrm{~s}$ and $\mathrm{Ts}=16 \mathrm{~s}$................5-3

Figure 5-5 Mean $\mathrm{V}_{\text {video }}$ plotted against mean $\mathrm{V}_{\mathrm{gt}}$ with $\mathrm{T}_{\mathrm{w}}=49 \mathrm{~s}$ and $\mathrm{Ts}=24.5 \mathrm{~s}$.............5-3

Figure 5-6 Mean $\mathrm{V}_{\text {video }}$ plotted against mean $\mathrm{V}_{\mathrm{gt}}$ with $\mathrm{T}_{\mathrm{w}}=64 \mathrm{~s}$ and $\mathrm{Ts}=32 \mathrm{~s}$...............5-4

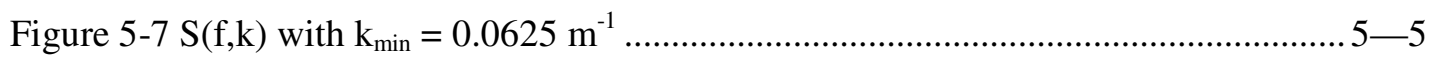

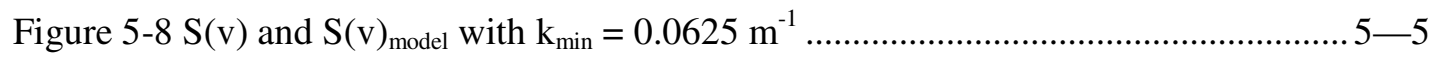

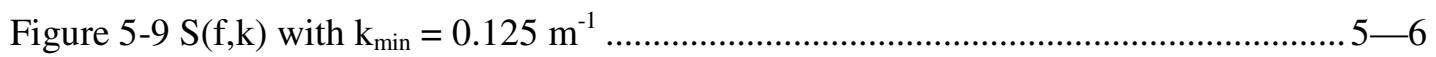

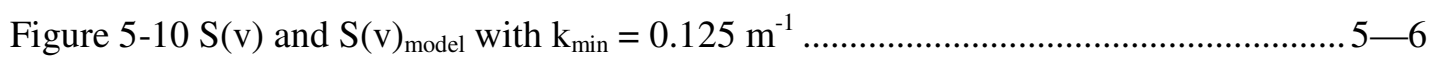

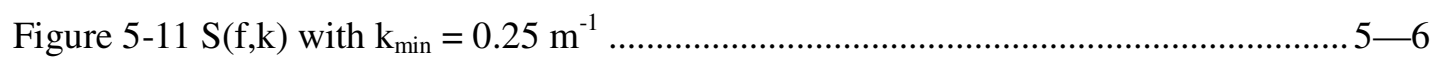

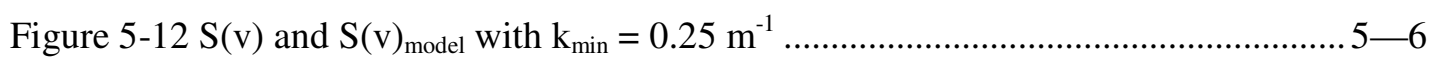

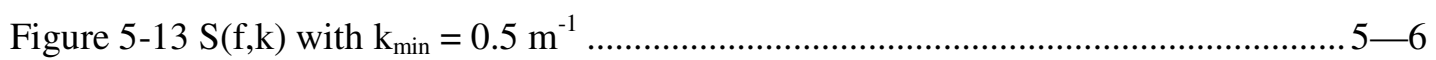

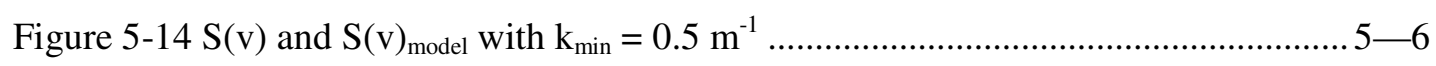

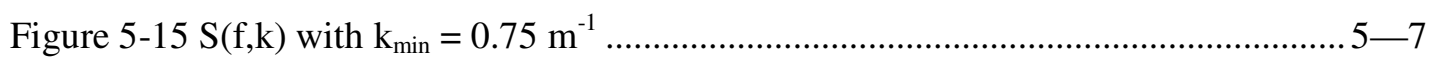

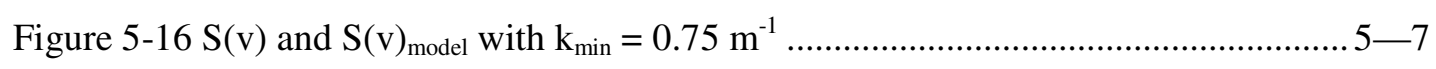

Figure 5-17 Mean $\mathrm{V}_{\text {video }}$ plotted against mean $\mathrm{V}_{\mathrm{gt}}$ with $\mathrm{k}_{\min }=0.0625 \mathrm{~m}^{-1}$......................5-7

Figure 5-18 Mean $\mathrm{V}_{\text {video }}$ plotted against mean $\mathrm{V}_{\mathrm{gt}}$ with $\mathrm{k}_{\min }=0.1250 \mathrm{~m}^{-1}$...................... 5-7

Figure 5-19 Mean $\mathrm{V}_{\text {video }}$ plotted against mean $\mathrm{V}_{\mathrm{gt}}$ with $\mathrm{k}_{\min }=0.2500 \mathrm{~m}^{-1}$.....................5-8

Figure 5-20 Mean $\mathrm{V}_{\text {video }}$ plotted against mean $\mathrm{V}_{\mathrm{gt}}$ with $\mathrm{k}_{\min }=0.5000 \mathrm{~m}^{-1} \ldots \ldots \ldots \ldots \ldots \ldots \ldots . . . . . . . . . .1-8$

Figure 5-21 Probability of the model fit for each individual velocity estimate $v_{i}$ plotted

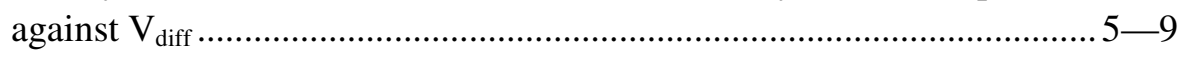

Figure 5-22 Percentage of data points with a certain probability ....................................5-9

Figure 5-23 Stack averaged probablity plotted against time ………...............................5-9

Figure 5-24 Mean $\mathrm{V}_{\text {video }}$ plotted against mean $\mathrm{V}_{\mathrm{gt}}$ with Probability $=0.1 \ldots \ldots \ldots \ldots \ldots \ldots \ldots . . . . .5-10$

Figure 5-25 Mean $\mathrm{V}_{\text {video }}$ plotted against mean $\mathrm{V}_{\mathrm{gt}}$ with Probability $=0.9 \ldots \ldots \ldots \ldots \ldots \ldots \ldots \ldots . . . . .5-10$

Figure 5-26 $\mathrm{CI}_{\mathrm{span}}$ for each timestep of each OCM timeseries plotted against $\mathrm{V}_{\text {diff } \ldots \ldots . . . .5-11}$

Figure 5-27 Mean $\mathrm{CI}_{\text {span }}$ per timeseries plotted against time.......................................... 5-11

Figure 5-28 Mean $\mathrm{V}_{\text {video }}$ plotted against mean $\mathrm{V}_{\mathrm{gt}}$ with C.I.span $=0.5 \mathrm{~m} / \mathrm{s} \ldots \ldots \ldots \ldots \ldots \ldots \ldots . . . . . . .12$

Figure 5-29 Mean $\mathrm{V}_{\text {video }}$ plotted against mean $\mathrm{V}_{\mathrm{gt}}$ with C.I.span $=0.2 \mathrm{~m} / \mathrm{s} \ldots \ldots \ldots \ldots \ldots \ldots . . . . . . . .12$

Figure 5-30 Mean $\mathrm{V}_{\text {video }}$ plotted against mean $\mathrm{V}_{\mathrm{gt}}$ with C.I.span $=0.1 \mathrm{~m} / \mathrm{s} \ldots \ldots \ldots \ldots \ldots \ldots \ldots . . . . . . . .12$

Figure 5-31 Mean $\mathrm{V}_{\text {video }}$ plotted against mean $\mathrm{V}_{\mathrm{gt}}$ with C.I.span $=0.05 \mathrm{~m} / \mathrm{s}$.................5-12

Figure 5-32 $\mathrm{I}_{\text {range }}$ for each timestep of each OCM timeseries plotted against $\mathrm{V}_{\text {diff }} \ldots \ldots . . . .5-13$

Figure 5-33 Mean $\mathrm{V}_{\text {video }}$ plotted against mean $\mathrm{V}_{\mathrm{gt}}$ with $\mathrm{I}_{\text {range }}=10 \ldots \ldots \ldots \ldots \ldots \ldots \ldots \ldots \ldots \ldots \ldots . . . .5-14$

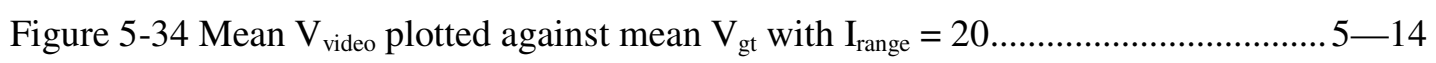

Figure 5-35 Mean $\mathrm{V}_{\text {video }}$ plotted against mean $\mathrm{V}_{\mathrm{gt}}$ with $\mathrm{I}_{\text {range }}=30 \ldots \ldots \ldots \ldots \ldots \ldots \ldots \ldots \ldots \ldots \ldots . . . . . . . . . . . . . . . .14$ 


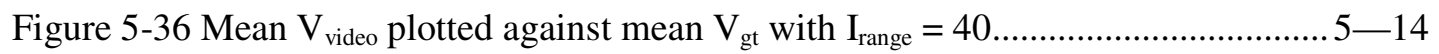

Figure 5-37 Mean $\mathrm{V}_{\text {video }}$ plotted against mean $\mathrm{V}_{\mathrm{gt}}$ with $\mathrm{I}_{\text {range }}=50 \ldots \ldots \ldots \ldots \ldots \ldots \ldots \ldots \ldots \ldots \ldots . . . .5-15$

Figure 5-38 Mean $\mathrm{V}_{\text {video }}$ plotted against mean $\mathrm{V}_{\mathrm{gt}}$ for the reference run .........................5-16

Figure 5-39 Mean $\mathrm{V}_{\text {video }}$ plotted against mean $\mathrm{V}_{\mathrm{gt}}$ for the final run...............................5-16

Figure 6-1 Pixel arrays at Noordwijk used for the analysis of the cross-shore distribution of the longshore current. The blue dots show the points in which the estimates

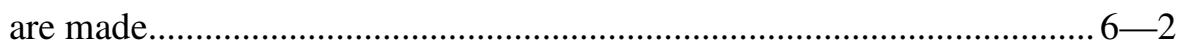

Figure 6-2 Cross-shore distribution of the longshore current velocity ............................6-2

Figure 7-1 Electronics container on a measuring frame ............................................... C-2

Figure 7-2 EMF current meter attached to the frame …………................................... C-2

Figure 7-3 Leaden plate attached to the foot of a frameleg ............................................ C-2

Figure $7-4$ Anchor to secure the frame position............................................................ 


\section{List of Tables}

Table 2-1 Measuring period Egmond field experiment divided in accordance with three different data collection schemes........................................................ 2-6

Table 3-1 Values of the sampling and model parameters for the Duck site ....................... 3-7

Table 4-1 Quality values for the OCM model runs with the Egmond and the Noordwijk data set, using a Duck-based default setting for the model parameters........... 4-3

Table 4-2 Quality values for different values of $\Delta y$ at Noordwijk .................................... $4-4$

Table 4-3 Quality values for different values of $\Delta \mathrm{y}$ at Egmond ........................................ 4-6

Table 5-1 Default setting of the model parameters ............................................................ 5-1

Table 5-2 Quality values of the reference run of model parameter analysis ...................... 5-2

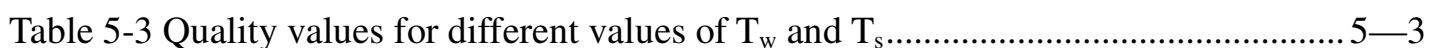

Table 5-4 Quality values for different values of the minimum wavenumber ....................5-7

Table 5-5 Quality values for different values of a threshold for the probability of the model fit

Table 5-6 Quality values for different values of the maximum confidence interval of the mean velocity of the model fit $5-11$

Table 5-7 Quality values for different values of the minimum intensity range ..............5-13

Table 5-8 Values of the model parameters used in the final run......................................5-15

Table 5-9 Quality values for the default and the final run ..............................................5-16 


\section{List of Symbols}

\begin{tabular}{ll} 
Roman symbols & \\
\hline $\mathrm{f}$ & focal length \\
$\mathrm{f}$ & frequency \\
$\mathrm{i}$ & imaginary unit $=\sqrt{-1}$ \\
$\mathrm{k}$ & wavenumber \\
$\mathrm{t}$ & time \\
$\mathrm{u}$ & horizontal image coordinate \\
$\mathrm{v}$ & vertical image coordinate \\
$\mathrm{v}$ & velocity \\
$\bar{v}$ & mean velocity \\
$\mathrm{x}$ & horizontal cross-shore field coordinate \\
$\mathrm{y}$ & horizontal longshore field coordinate \\
$\mathrm{Z}$ & vertical field coordinate \\
$\mathrm{A}$ & peak value \\
$\mathrm{B}$ & Bartlett multiplicative filter \\
$\mathrm{C}$ & covariance \\
$\mathrm{C} . \mathrm{I}$. & $95 \%$ confidence interval of the mean velocity \\
$\mathrm{G}$ & 'grassiness' parameter \\
$\mathrm{I}$ & pixel intensity \\
$\mathrm{I}_{\mathrm{range}}$ & difference between $\mathrm{I}_{50}$ and $\mathrm{I}_{95}$ \\
$\mathrm{I}_{50}, \mathrm{I}_{95}$ & $50^{\text {th }}$ and $95^{\text {th }}$ percentile intensity values \\
$\hat{I}$ & Fourier transformed intensity \\
$\mathrm{L}$ & linear function of camera orientation, position and focal length \\
$\mathrm{M}$ & merit function \\
$\mathrm{P}$ & probability of the model fit \\
$\mathrm{S}$ & time shift \\
$\mathrm{T}_{\mathrm{s}}$ & time window \\
$\mathrm{T}_{\mathrm{w}}$ &
\end{tabular}

\section{Greek symbols}

$\begin{array}{ll}\beta & \text { parameter space of the model } \\ \hat{\beta} & \text { best-fit solution of } \beta \\ \gamma & \text { correlation coefficient } \\ \delta & \text { horizontal field of view } \\ \mu & \text { mean } \\ \pi & \text { pi }=3,1416 \\ \sigma & \text { roll }\end{array}$




$\begin{array}{ll}\sigma & \text { standard deviation } \\ \tau & \text { tilt } \\ \chi^{2} & \text { statistical chi-square test on model fit } \\ \Delta & \text { difference } \\ \Delta y & \text { sampling spacing } \\ \phi & \text { azimuth }\end{array}$

\section{Superscripts}

complex conjugate

\section{Subscripts}

c

foam

gt

$\mathrm{m}$

mean

$\min$

noise

nyq

rms

video

$\mathrm{X}$

y at camera location

of foam patches

ground truth

model

mean

minimum

background noise

Nyquist

root-mean-square

from a video image

cross-shore

longshore 


\section{Summary}

Longshore currents drive changes of coastal morphology and also embody a potential threat for swimmer safety. This explains the interest of coastal managers and scientists in costefficient techniques to quantify alongshore flow patterns. Traditional in-situ methods like Electro Magnetic (EMF) flow meters are expensive, time-consuming, dangerous to deploy during rough wave conditions and lack synoptic coverage. Recently a new technique was developed which quantifies flow velocities from intra-wave video observations of the nearshore (Chickadel et al., 2003). This Optical Current Meter (OCM) was successfully tested against extensive field experiments at a swell-dominated, intermediate to reflective beach at Duck, NC (USA). In this thesis, we investigate the applicability of the OCM at a dissipative beach at Egmond and Noordwijk (the Netherlands), characterized by shorter waves and a mild beach slope.

\section{The Optical Current Meter (OCM)}

The OCM was developed at Oregon State University as part of the Argus video program (Holman et al., 1993). It is based on the analysis of short time series of image intensities, sampled from an alongshore array of pixels (Figure 1-3). This yields alongshore time stack images (Figure 1-1), which typically reveal the bright horizontal bands of passing breaking waves and the oblique traces of foam patches drifting with the prevailing alongshore current. The velocity of the foam patches gives a measure of the alongshore surface current velocity. To quantify the surface foam drift, intensity time stacks are first Fourier transformed to a frequency-wavenumber spectrum, and finally to a velocity distribution. A model of the velocity distribution is fit to the observed distribution to estimate the foam drift velocity. Field test comparisons against an in-situ bidirectional EM flow meter, involving one month of video data sampled during the 1997 SandyDuck experiment, showed an $r m s$ error of $0.10 \mathrm{~m} / \mathrm{s}$.

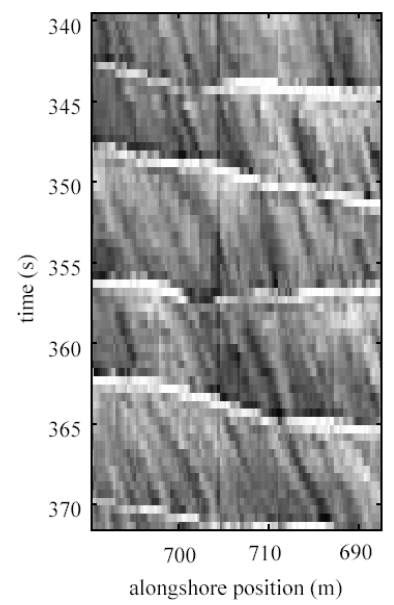

Figure 1-1 Longshore intensity time-stack, collected during SandyDuck (1997)

OCM application in Egmond and Noordwijk (The Netherlands)

To verify the generic applicability of the OCM, the model was applied to quantify alongshore flow velocities at two field sites along the Dutch coast. The first test, performed 
at Egmond, involved a comparison of video-derived flow velocities and ground-truth flow meter measurements, both collected at $500 \mathrm{~m}$ north of the video station. At this distance, alongshore pixel resolutions are in the order of $2 \mathrm{~m}$. This resolution turned out to be insufficient to resolve foam patches, which explains poor OCM performance at Egmond.

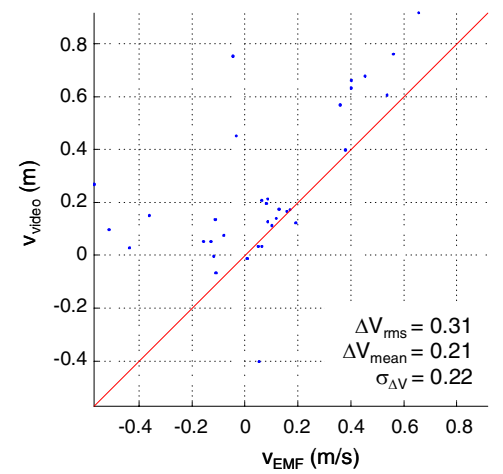

Figure 1-2 OCM model validation against EMF-measured velocities at Noordwijk

The second test involved the validation of OCM against a two-week dataset of hourlymeasured alongshore flow velocities, sampled directly in front of the Noordwijk video station. With an rms off-set of about $30 \mathrm{~cm}$, the technique shows improved performance (Figure 1-2). For positive (i.e. southward-directed) velocities, video-derived velocities consistently overestimate the corresponding EMF-measured values. This can be explained from the observation that the OCM estimates surface velocities, whereas the EMF measures a velocity in the water column, at about $25 \mathrm{~cm}$ above the sea bed.

To illustrate the potential of the OCM, the model was used to quantify flow-velocities along five cross-shore arrays spacing $10 \mathrm{~m}$ cross-shore. The result (Figure 1-3) shows a realistic distribution of flow velocities across the surf zone, with maximum velocities up to $0.65 \mathrm{~m} / \mathrm{s}$ at about $40 \mathrm{~m}$ off the shoreline.
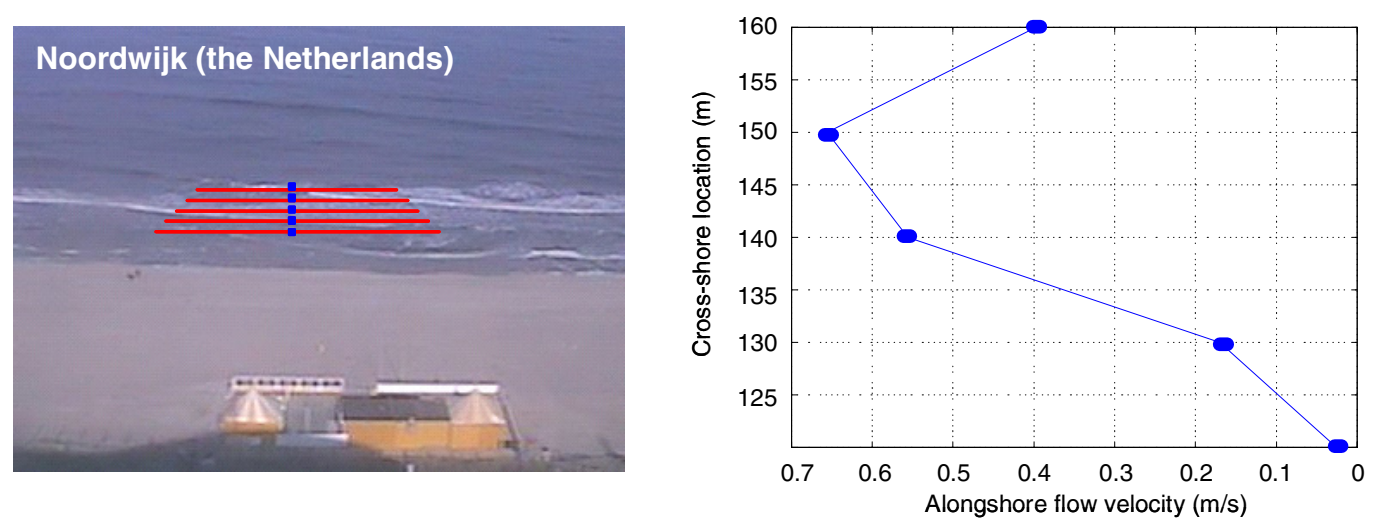

Figure 1-3 Video-derived cross-shore distribution of alongshore flow velocities at Noordwijk

The OCM has proved to provide usable results at the Holland coast within a range of $200 \mathrm{~m}$ at both sides of a camera station. Improvements in camera resolution will also improve the usability of the OCM. 


\section{Introduction}

\section{I.I General}

\section{I.I.I Introduction}

Why study longshore currents and why study video techniques? The present research is desired from two different directions. The first is the research of longshore currents. These play a role in beach recreation, because large flow velocities in longshore direction can cause danger to swimmers. It is thus important to get a feeling of the current velocities in the swimming area to assure safety for swimmers. Furthermore, longshore currents are the major cause of longshore sediment transport in the surf zone. It is important to quantify the longshore sediment transport rate to be able to understand and predict morphological changes. Currently, longshore current velocities are mostly measured using Electro Magnetic Flow meters (EMF), which have to be attached to frames and placed in the water. This method is very labour intensive, time-consuming, dangerous during rough wave conditions and only yields a single point measurement in space. This brings us to the second motivation for this research. Recently, a technique has been developed that uses pixel intensity time series to obtain longshore current velocities. This technique is one of the applications of a broader used video remote sensing programme, named Argus (Holman et al., 1993). The pixel intensity time series technique is still in a very early stage of research and has not yet been applied to other locations than the first test location, Duck USA.

\section{I.I.2 Longshore currents}

Longshore currents are common features on most coasts. The nearshore current velocity field in longshore direction is strongly influenced by tide, wind and wave driven processes.

\section{Tide}

The tide is the alternating rise and fall of sea level with respect to land, as influenced by the gravitational attraction of the moon and sun. Other factors influence tides, such as coastline configuration, local water depth, bathymetry, winds, and weather conditions. These factors alter the arrival times of tides and water level interval between high and low water. The tide can generate a longshore current when the tidal wave is directed parallel to the coast. The flood current is directed in the propagation direction of the tidal wave, the ebb current is directed in the opposite direction.

\section{Wind}

When the wind blows along the sea surface, it causes both surface currents and waves. Several semi empirical observations are useful. One is that the velocity of the surface current induced by a wind is approximately $3 \%$ of the wind speed. One might expect a 0.3 
$\mathrm{m} / \mathrm{s}$ velocity of the surface current with a $10.3 \mathrm{~m} / \mathrm{s}$ wind speed. The second is that the stress applied to the sea surface increases as the square of the wind speed (Knaus, 1978).

\section{Waves}

Waves propagating onshore induce a radiation stress, which is the wave's contribution to the horizontal transfer of horizontal momentum per unit width, through a vertical plane from bottom to water surface and averaged over the wave period. When waves approach the coast at an angle, radiation shear stresses are generated. Cross-shore gradients in this radiation shear stress occur when the waves lose energy, due to breaking. These gradients produce the driving force which acts parallel to the coast and drives the water mass along the coast, thus generating the wave driven longshore current. Outside the breaker zone the radiation shear stress is constant in cross-shore direction. This is why longshore currents typically occur in the breaker zone.

\section{I.I.3 Measuring longshore currents}

Up to now, current velocities are usually measured using current meters. These devices are usually mounted on a frame, which has or has not got an electronics container attached to it. When the frame does not have an electronics container, thus when it is not a stand-alone frame, cables have to provide energy for the meters and have to transfer the measured data from the measuring device to a computer situated onshore. When stand alone frames are used (see Figure 1-1), the frames have to be removed from the water almost every day to download the measured data and to check the energy supply (batteries). Both methods are very time and labour intensive.

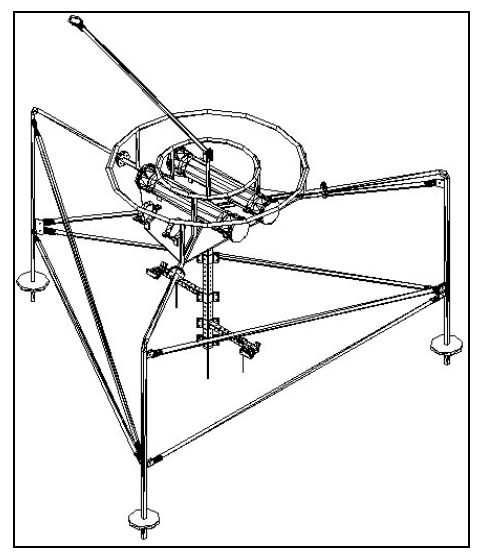

Figure 1-1 An example of a tripod stand alone measuring frame

\section{I.2 Framework of this research}

\section{I.2.I The CoastView project}

The present study has been carried out in the framework of the CoastView project. This project focuses on the physical problems associated with sedimentary coasts and aims to support the coastal manager who needs to know when a valuable component of the coast is 
at risk, which processes are responsible and what appropriate form of intervention is required in order to sustain or improve that component. The CoastView project has two primary objectives:

- To develop resource-related Coastal State Indicators (CSIs) for describing the dynamic state of the coast, in support of coastal zone management.

- To develop and verify video-based monitoring methods and associated analysis techniques for estimating and interpreting these CSIs.

CSIs can be defined as a minimum set of parameters that can quantitatively describe the dynamic state of a coastal system.

The CoastView project looks at four morphologically dissimilar field sites that collectively typify the sort of management issues that are common to European coastlines. These sites include a continuous-undefended coastline (Egmond, Netherlands), a continuous-defended coastline (Lido di Dante, Italy), a coastal inlet with a single bar or spit (El Puntal, Spain) and a coastal inlet with multiple complex bars (Teignmouth, UK). Both generic CSIs will be developed as well as CSIs that apply specifically to certain environmental groupings. The coastal management is divided into four issues for which CSIs are specified:

- Coastal Safety \& Maintenance

- Recreation \& Tourism

- Shipping \& Navigation

- Natural values

The longshore current velocities play a role as CSI in one of these issues, i.e. Recreation \& Tourism. The longshore current velocities indicate if it is safe to swim and thus if the beach in question is suitable for tourism. The fact that longshore current velocities play a major role in longshore sediment transport processes is not mentioned in the CSI list of the CoastView project, but knowing the value of longshore current velocities to calculate the longshore sediment transport is of great importance in the prediction of morphological changes.

The second objective of the CoastView project focuses on the development and verification of video-based monitoring methods and associated analysis techniques for estimating and interpreting these CSIs. The present study concentrates on pixel intensity time series analysis to estimate longshore current velocities.

\subsubsection{The Argus research program}

\section{Argus video imaging}

The coastal zone is probably the most dynamic part of any oceanic domain. Given the dense populations living in this type of area, it is of great importance to inspect and protect the coastal system intensively. Inspection can be very costly and infrequent, while a storm can cause significant coastal changes in only a few hours. The Argus video program, developed at the Coastal Imaging Lab, Oregon State University, offers a way to inspect the coastal 
zone almost continuously. This program involves the installation of unmanned, automated video stations at sites of research or management interest. Each Argus station consists of five video cameras, offering a 180 degrees panoramic view of the coastal area and allowing full coverage of about 3 to 5 kilometres of beach.

Video data are collected during daylight and transmitted to a local archive by phone line. The process of data collection and return is fully automated. From the local archive, the data are transmitted by phone line or through the internet to an Argus archive server elsewhere, were all the collected data are stored. The most used data from the Argus server are put on an internet site, so that the data are accessible from anywhere around the world. Stations are currently located in the places named in Appendix A.

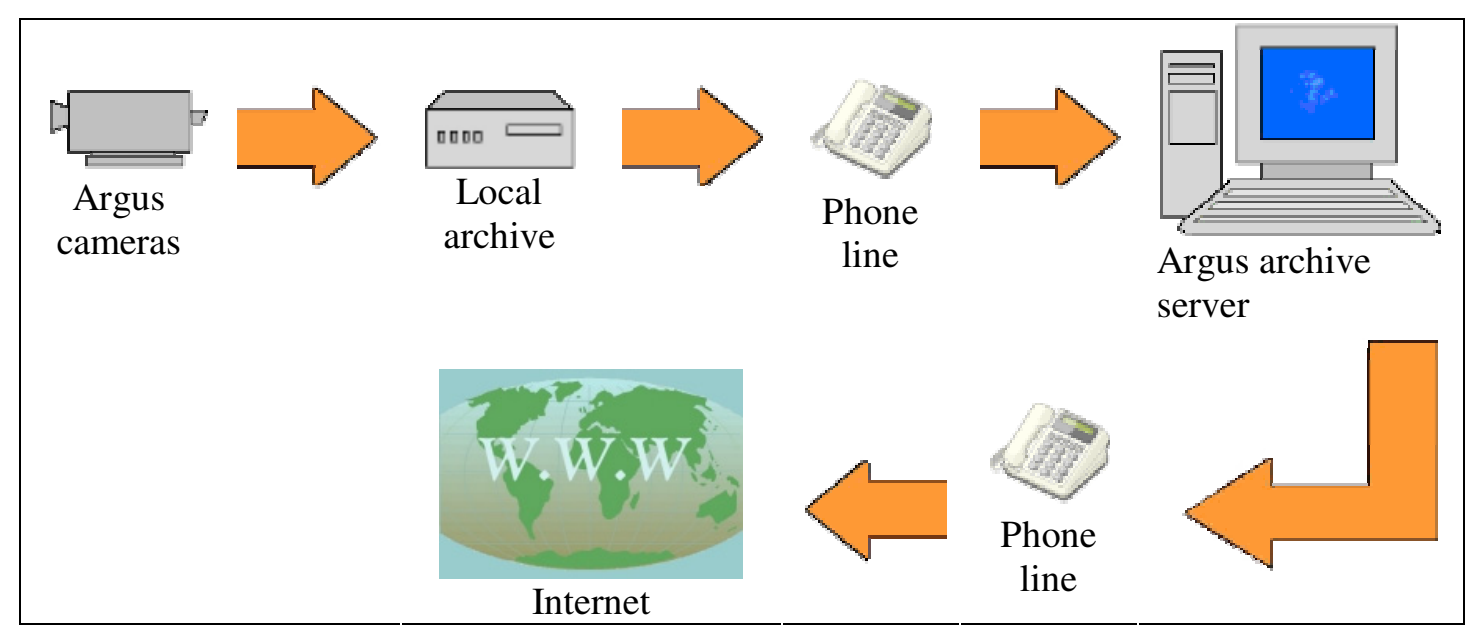

Figure 1-2 Data transmission from the Argus camera to the internet

At every Argus site, the orientation of the $\mathrm{x}$-axis is shore normal, with the positive $\mathrm{x}$-axis pointing in seaward direction. The $y$-axis is directed perpendicular to the $x$-axis, such that the co-ordinate system thus obtained is positive in a mathematical sense (see Figure 1-3). The latter means that the rotation from the $\mathrm{x}$-axis towards the $\mathrm{y}$-axis indicates the counterclockwise or positive direction. The horizontal reference level $(\mathrm{z}=0)$ is generally set to match the mean tidal level or a commonly used ordinance level (like NAP in The Netherlands). Presently three types of images are collected from the Argus cameras, i.e. snapshots, time exposure images and variance images. Furthermore pixel intensity time series can be generated, which show the fluctuations in time of the intensity in a pixel. 


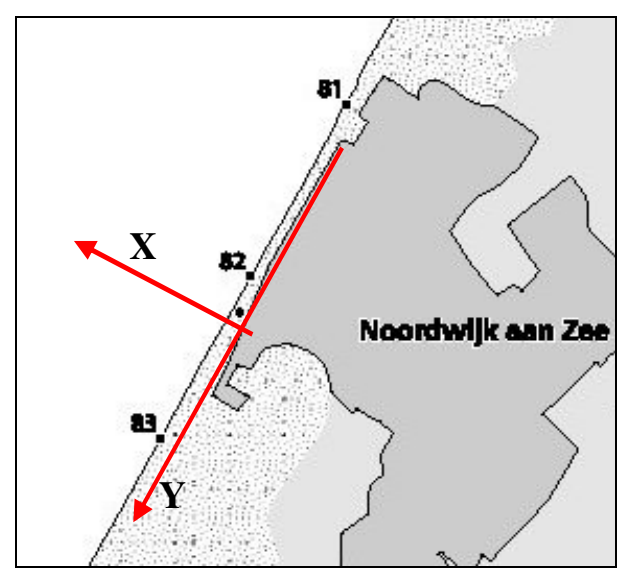

Figure 1-3 Example of the Argus co-ordinate system (Noordwijk aan Zee)

Quantification of image features like sand bars, shorelines and foam patterns demands the conversion of image coordinates $(u, v)$ to field coordinates $(x, y, z)$. This conversion depends on the camera location $\left(x_{c}, y_{c}, z_{c}\right)$, the camera orientation defined through three camera angles, namely the tilt $\tau$, the azimuth $\phi$ and the roll $\sigma$ (see Figure 1-4), and the effective focal length $f$, which directly relates to the camera horizontal field of view $\delta$. The angles $\tau, \phi$ and $\sigma$ represent the rotation with regard to the vertical $z$-axis, the orientation in the horizontal $x y$-plane and the rotation of the focal plane with respect to the horizon, respectively. The transformation of field to image coordinates is thus a rotation about three successive angles $\tau, \phi$ and $\sigma$ and can be expressed by the linearised version of the collinearity equations, following standard photogrammetric procedures, as follows (Holland et al., 1997):

$$
\mathrm{u}=\frac{\mathrm{L}_{1} x+L_{2} y+L_{3} z+L_{4}}{\mathrm{~L}_{9} x+L_{10} y+L_{11} z+1}
$$

and

$$
\mathrm{v}=\frac{\mathrm{L}_{5} x+L_{6} y+L_{7} z+L_{8}}{\mathrm{~L}_{9} x+L_{10} y+L_{11} z+1},
$$

The coefficients $\mathrm{L}_{1}$ to $\mathrm{L}_{11}$ are linear functions of the camera orientation $(\tau, \phi, \sigma)$, the camera position $\left(x_{c}, y_{c}, z_{c}\right)$ and the effective focal length $(f)$ (see Appendix B). The inverse transformation from image to field coordinates results in a system of two equations with three unknowns. The z-coordinates are assumed in this transformation to match a certain horizontal reference level or the tidal water level. 


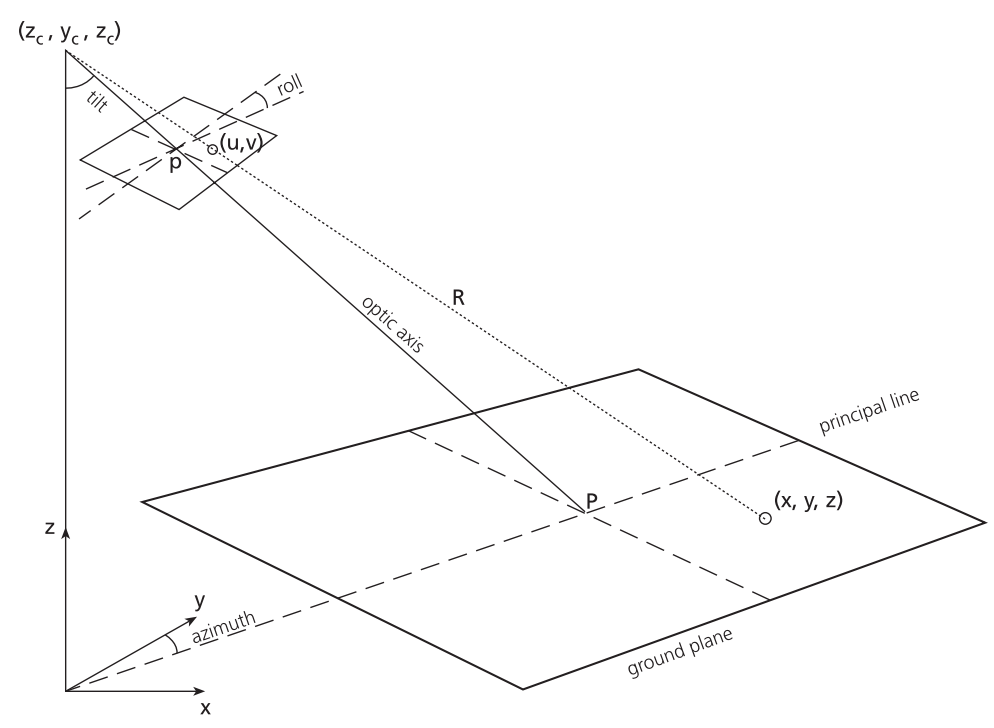

Figure 1-4 Relation between image $(\mathrm{u}, \mathrm{v})$ and real world $(\mathrm{x}, \mathrm{y}, \mathrm{z})$ co-ordinates

\section{Video data types}

Three types of images are gathered from the Argus cameras (see Figure 1-5, Figure 1-6 and Figure 1-7). Every hour, a snapshot, a time exposure and a variance image are collected for each of the stations cameras. A snapshot serves as simple documentation of conditions, but offers little quantitative information. The time exposure images provide us with much more information. The ten minute time exposures of the nearshore wave field average out natural modulations in wave breaking to reveal a smooth band of white which has been shown to be an excellent proxy for the underlying, submerged sand bar topography (Lippman and Holman, 1989 and Van Enckevort and Ruessink, 2001). The third type, the variance image, displays the variance of the light intensity signal during the same ten minutes of time exposure. Variance images help identify regions which are changing in time, from those, which may be bright in the time exposure, but are unchanging in time.

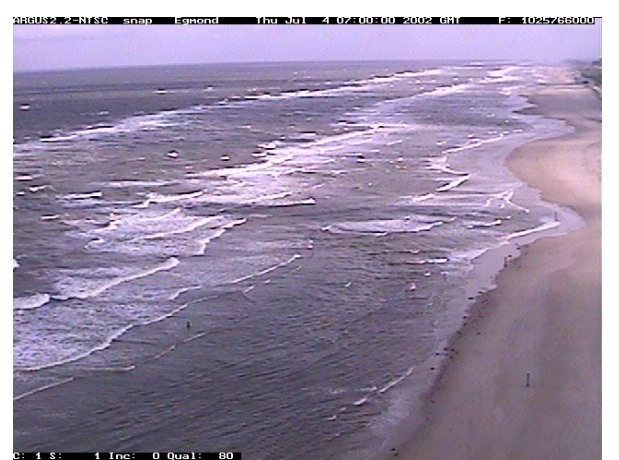

Figure 1-5 A snapshot image at Egmond aan Zee

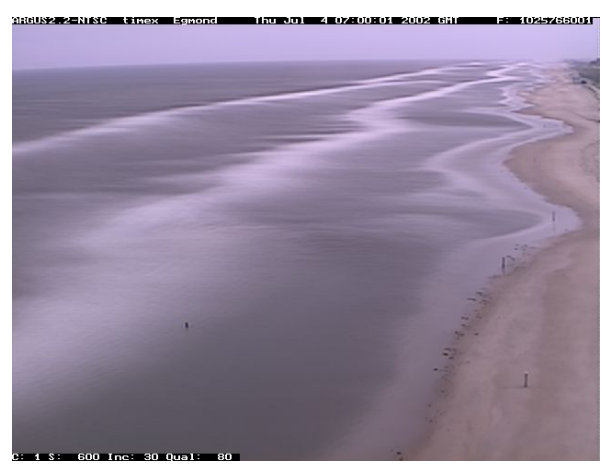

Figure 1-6 A time exposure image at Egmond aan Zee 


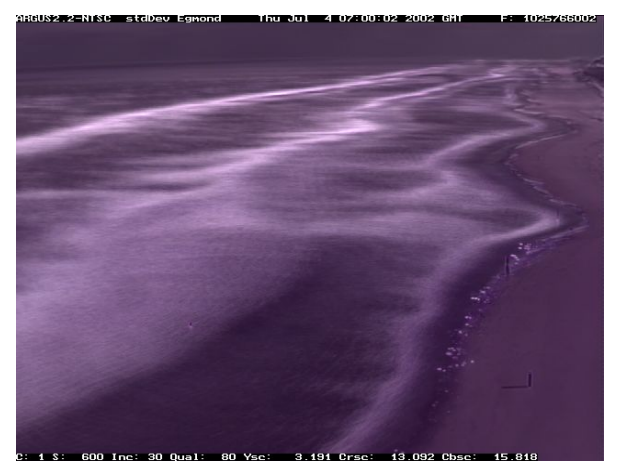

Figure 1-7 A variance image at Egmond aan Zee

The collection and analysis of pixel intensity time series presently attract considerable attention. Pixel intensity time series show the fluctuations in time of the intensity in a pixel. A time stack image is an image in which the intensity of an array of pixels is plotted against time. This information is already being used to calculate wave phase speed from which the water depth can be estimated. The present research is carried out to extend the analysis of pixel intensity time series to obtain longshore current velocities.

\section{The time stack image}

Besides snap shots and time-averaged video data, data sampling schemes can be designed such to collect time series of image intensities, typically at $2 \mathrm{~Hz}$. To illustrate, Figure 1-8 shows the strong correlation which is commonly observed between a time series of pixel intensities and the wave height signal obtained from a wave gauge at the same location (Lippmann and Holman, 1991). The small phase lag between the two indicates that maximum intensities correspond to the white, foam-covered face of the breaking wave, which precedes the passage of the actual wave top. Comparisons for non-breaking waves also show strong coherence, but often a larger, still fixed, phase difference.
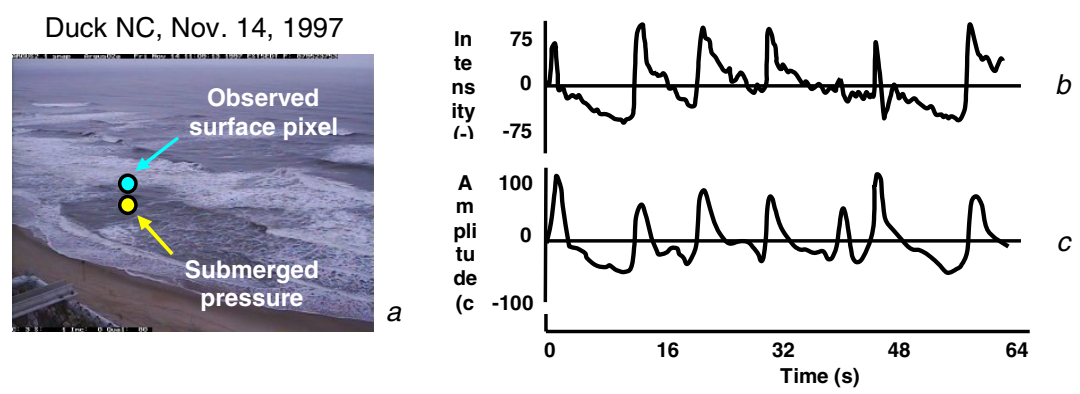

Figure 1-8 Relation between a pixel intensity time series and a wave height signal at Duck, USA

In extension of this approach, time series of pixel intensities can be sampled along a crossshore or alongshore array. The resulting data collection, I $(\mathrm{t}, \mathrm{y} ; \mathrm{x})$, yields a time stack image (see Figure 1-9). Due to the digitalisation process, the intensity data are normalised over the lens's intensity range and thus dimensionless. The dark, slightly curved patterns represent individual waves propagating onshore. The slope of the wave traces can be used to determine the approximate speed of the shoreward progressive waves, before being dissipated through wave breaking at the shoreline (around $\mathrm{x}=170 \mathrm{~m}$ ). 


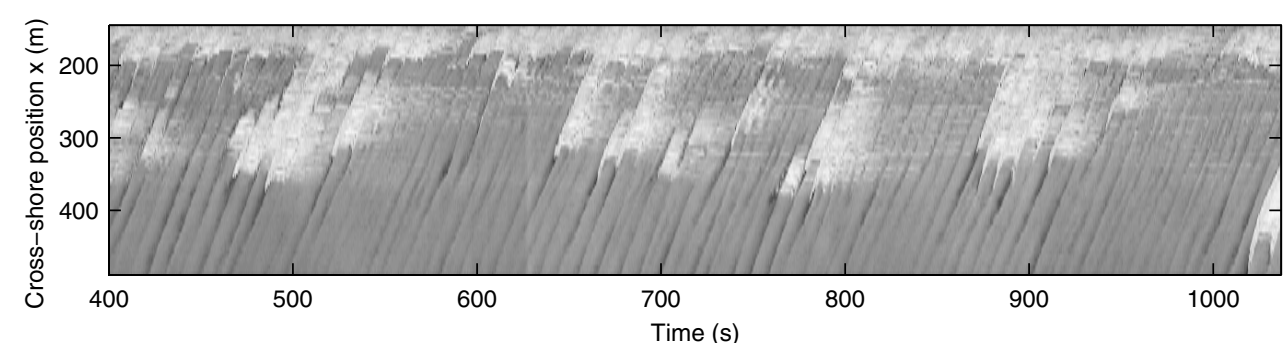

Figure 1-9 A time stack image collected along a cross-shore array at Duck, USA

The work presented in this thesis is based on the use of alongshore sampled time stack images (see Figure 1-10 and Figure 1-11) from which the longshore current velocity is estimated. The targets for the longshore current analysis are the foam patches that appear on the water surface after the waves have broken on the nearshore bar. These foam patches move slowly alongshore carried by the longshore current. The velocity of the foam patches therefore gives a measure of the longshore surface current velocity. The model to estimate the velocity of the foam patches will be explained in Chapter 3. The longshore current velocity time stack analysis has until now only been used with data from a field experiment in Duck, North Carolina, USA. The analysis has been carried out at the Oregon State University by C.C. Chickadel. His research is focussed at the Duck site and contains several site specific aspects.

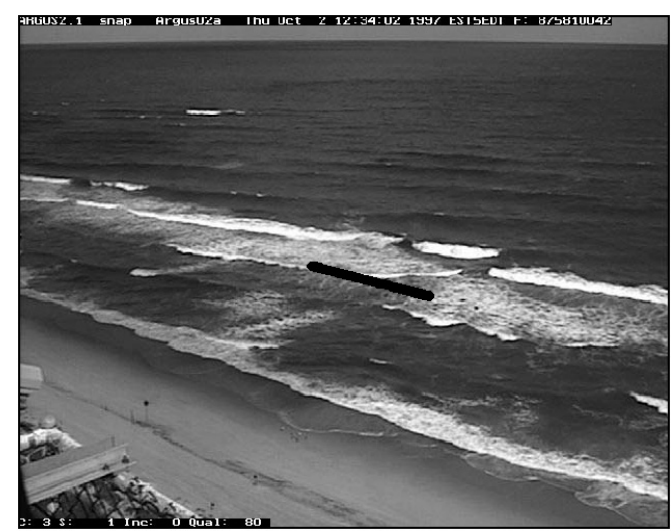

Figure 1-10 Snapshot of the surf zone at Duck NC. The line indicates the location of the alongshore pixel array where the video record was taken.

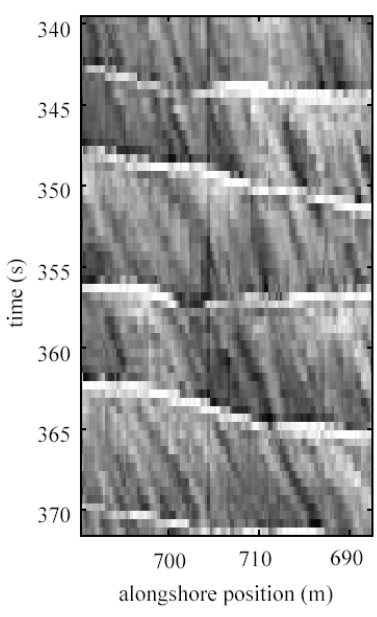

Figure 1-11 A time stack, collected at Duck NC. The time stack reveals the bright horizontal bands of passing breaking waves and the oblique traces of foam patches drifting with the prevailing longshore current.

\section{I.3 Research objectives and outline of this thesis}

\section{I.3.I Objectives}

This research is motivated by two issues, i.e. (i) the demand for a less labour intensive and cheaper method to measure longshore current velocities, to improve our understanding of 
longshore current dynamics at the coast, and (ii) the ongoing development of methods to quantify physical variables from video imagery, particularly time stack applications. The central aim of this work thus is to quantify longshore current velocities from time stack images collected along the Holland coast. Three main objectives are pursued to achieve this central aim.

- To study the applicability of the longshore current velocity time stack analysis, which has until now only been applied to the Duck site in the USA. To achieve this, the analysis method will be applied to two sites in The Netherlands, i.e. Egmond aan Zee and Noordwijk aan Zee.

- To determine the accuracy of this analysis method through calibration and validation against a set of ground truth data, obtained during two field experiments in Egmond aan Zee (2002) and Noordwijk aan Zee (2003).

- To assess the utility of the longshore current velocity time stack analysis for application in support of coastal management and science.

The three key terms of the research thus are the applicability, accuracy and utility of the longshore current velocity time stack analysis. These terms will come back in the analysis part and the concluding part of this report.

\section{I.3.2 Outline}

In this introductory Chapter 1, background information is given that motivates the present research and the resulting objectives of the research are explained. Chapter 2 focuses on the two field experiments that have been carried out in the framework of this research. Information is given about the sites and the measuring techniques used to obtain data. Finally, the data sets for each site are discussed. The model that will be used in the longshore current velocity time stack analysis is explained in Chapter 3. Chapter 4, 5 and 6 go into the three key terms of this research, i.e. applicability, accuracy and utility. In Chapter 4, the same model is used for the analysis of the Egmond and Noordwijk data as during the analysis of the Duck data. With this, the applicability of the model to sites other than the Duck site is studied. The accuracy of the model output is discussed in Chapter 5, where a calibration and validation against ground truth data is carried out. This results in a best setting of the model parameters for the tested sites. Finally, a discussion on the utility of the model is presented in Chapter 6, where we will go into four different aspects of utility. The last chapter of this thesis reports the conclusions that can be drawn with respect to the three key terms mentioned earlier. Also, recommendations are given, so that further research concerning longshore current velocity time stack analysis will be fruitful. 


\section{Field sites: Egmond and Noordwijk}

\section{I Introduction}

Two field experiments have been carried out in the framework of this study to provide data for the longshore current velocity time stack analysis. The first experiment has taken place in Egmond aan Zee (see Figure 2-1) from October 14 until November 3, 2002. This site is chosen for the reason that it was already part of the CoastView project (see section 1.2.1) and that an Argus station is present at the beach. The second experiment has been carried out in Noordwijk aan Zee (see Figure 2-2) from April 4 until April 16, 2003. Noordwijk has hosted an Argus station since 1995 on the top of hotel "Huis Ter Duin".

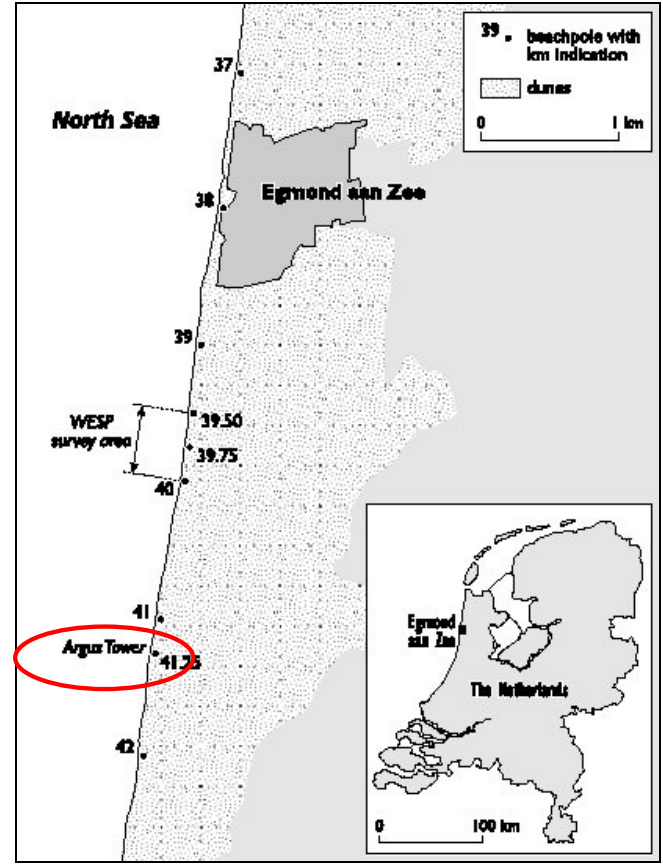

Figure 2-1 Map of Egmond field site

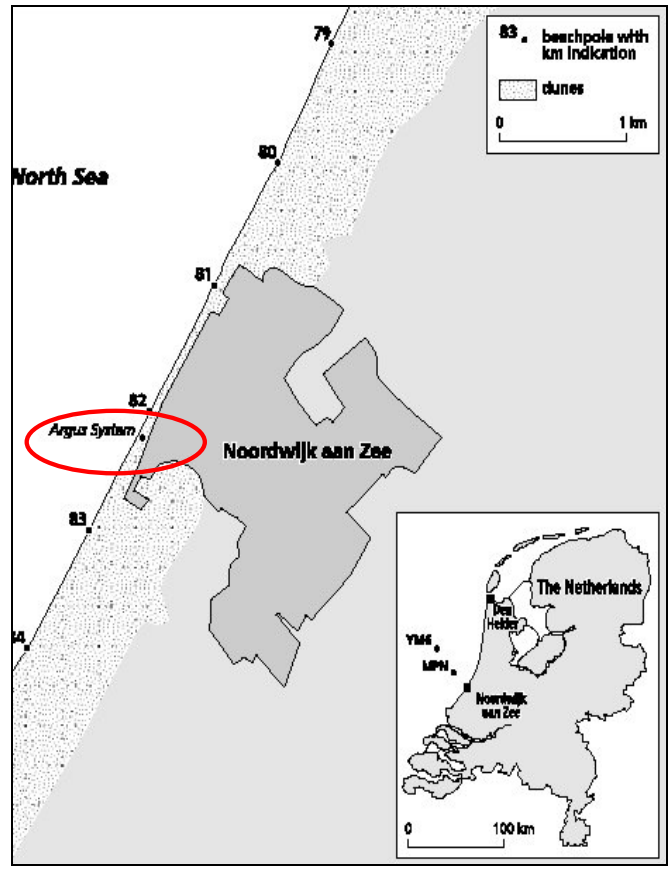

Figure 2-2 Map of Noordwijk field site

\subsection{Site information}

\subsection{The Holland coast}

The coastal zone of the Netherlands can be divided into three major regions, the Delta area, the Wadden area and the Holland coast. These regions differ both in morphological appearance and in the dominance of related physical processes. The first experiment, carried out between October $14^{\text {th }}$ and November $3^{\text {rd }}, 2002$, took place near Egmond aan Zee, the Netherlands, which is located on the Holland coast, between the harbour moles of IJmuiden and the Hondsbossche Seawall. The second experiment took place in Noordwijk aan Zee, 
from April $4^{\text {th }}$ until April $16^{\text {th }}$ in the year 2003. Noordwijk aan Zee is located approximately 60 kilometers south-west of Egmond aan Zee, just north-east of Katwijk aan Zee.

\subsubsection{Geomorphology}

The Holland coast mainly consists of straight sandy beaches and multi-barred nearshore zones. The shore face has a gentle slope of about 1:400 with a transition to the North Sea bottom at NAP- $20 \mathrm{~m}$. The Egmond area has two nearshore bars, an inner bar and an outer bar. The crest of the outer bar is almost straight and lies at a level of NAP-3.5 to NAP-4.0 m. The crest of the inner bar has an irregular alongshore planview and lies at a level of NAP1.5 to NAP-2.5 m.. Frequently, rip channels appear in the inner nearshore bar with an

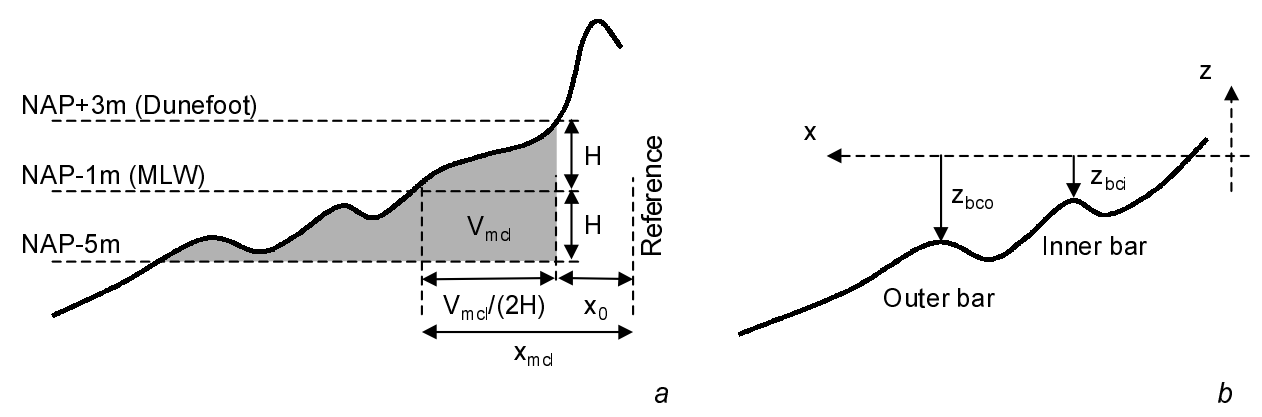

Figure 2-3 The bar system in the nearshore zone along the Holland coast

\subsubsection{Hydrodynamic conditions}

The coast near Egmond aan Zee and Noordwijk aan Zee is located along the southern part of the semi-enclosed North Sea and is hydrodynamically distinguished as a mixed-energy coast. This implies that both wind waves and tides determine the morphological characteristics in this area.

\section{Wind and waves}

The majority of the winds along the Holland coast come from the North Sea. The prevailing wind direction is south-west (23\%), followed by west (16\%), east (13\%) and north-west (12\%). The largest wind set-up along the coast is caused by storm winds coming from northwest. The seasonal variation in the strength of the wind climate is clearly reflected in the wave climate. During the stormy winter months, November until January, the monthly mean wave height is approximately $1.7 \mathrm{~m}$. During the more quiet summer months, April until August, the monthly mean wave height is about $1 \mathrm{~m}$ (Van Rijn et al., 2002). 


\section{Tide}

The tidal currents along the Holland coast are directed northward during the flood period and southward during the ebb period. The semi-diurnal tidal curve is asymmetrical with a flood period of 4 hours and an ebb period of 8 hours near Egmond aan Zee. This tidal asymmetry is mainly caused by an interaction between the M2 and M4 tidal components. The mean tidal range at Egmond has a value of $1.65 \mathrm{~m}$. The spring tidal range is $2.0 \mathrm{~m}$ and the neap tidal range is $1.4 \mathrm{~m}$ (Van Rijn et al., 2002). The tide in Noordwijk lies in the same range, with a neap tidal range of $1.4 \mathrm{~m}$ and a spring tidal range of $1.8 \mathrm{~m}$. Strong winds (not counting storms) may increase the offshore water level by up to $1 \mathrm{~m}$. The majority of the wind setup values, however, remains between $-0.5 \mathrm{~m}$ and $0.5 \mathrm{~m}$. The predicted tidal curves and tables for the Dutch coast can be found on the website: http://www.getij.nl. Because the tide in Egmond aan Zee is not presented on the site, the average between the water level in IJmuiden and in Petten Zuid is used as the tide in Egmond aan Zee.

\section{Longshore currents}

The maximum tidal longshore current velocities near the bed in the surfzone at Egmond aan Zee are in the range of 0.3 to $0.5 \mathrm{~m} / \mathrm{s}$. These conditions occur during calm weather conditions with significant offshore wave heights up to approximately $1 \mathrm{~m}$. The wind- and wave-induced longshore currents at the inner bar crest generally lie between 0.5 and $1.3 \mathrm{~m} / \mathrm{s}$ during storm conditions. Storms from the south-west strongly enhance the flood currents to the north, but reduce the ebb currents to the south. Storms from the north-west have the opposite effect. Roughly, it can be stated that the peak tidal currents are increased by a factor 3 to 4 due to wind- and wave-driven processes during major storm events. The largest velocities are found near the crests of the bars (Van Rijn et al., 2002). The same conditions can be expected along the Noordwijk coast.

\subsection{Argus stations}

In Chapter 1.2.2, the composition of an Argus station is discussed. At both field sites, an Argus camera station is present. The Egmond Argus station is located on a 40 meter high unmanned tower three kilometers south of the main Egmond beach entry. The station was installed in December, 1997. Because it is difficult and dangerous to access the tower, the cameras are mounted on a trolley and raised into their monitoring position. The site features five cameras, spanning 180 degrees of view. The Noordwijk station was installed in March, 1995, and also consists of five cameras, covering the same span as the cameras in Egmond. The station is located on the curved roof of hotel "Huis Ter Duin", the largest hotel in Noordwijk aan Zee. Images from both stations are collected on a computer at the site and transferred through a phone line to Argus servers at Utrecht University and WLIDelft Hydraulics, where the data are stored. Data can be copied from this server for analysis at any location. 


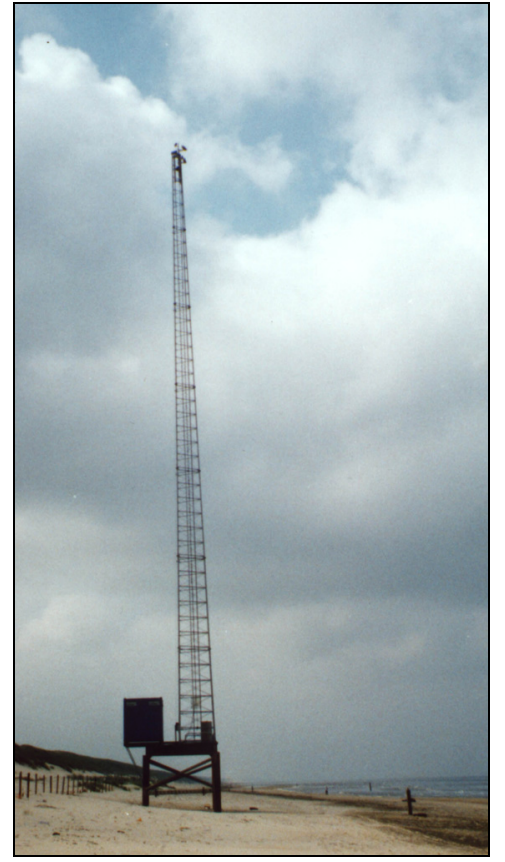

Figure 2-4 Coast3D tower with Argus station in the top at the Egmond beach

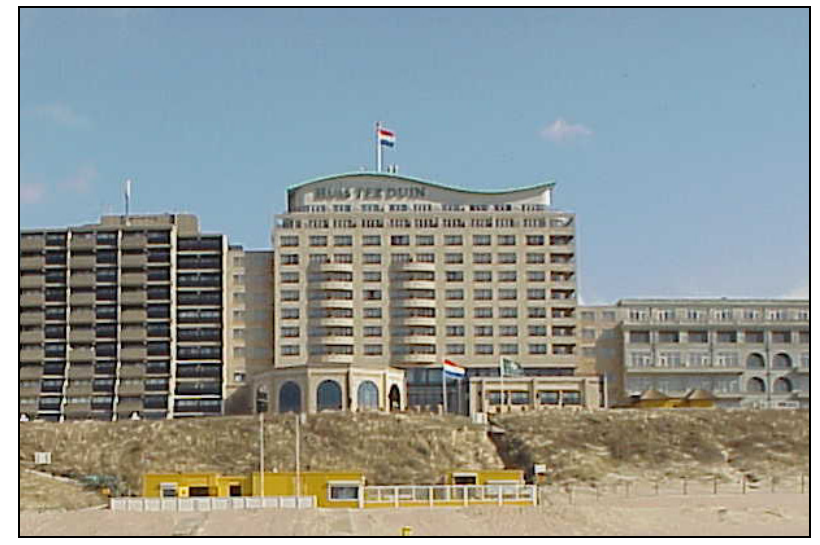

Figure 2-5 Hotel "Huis Ter Duin" in Noordwijk aan Zee

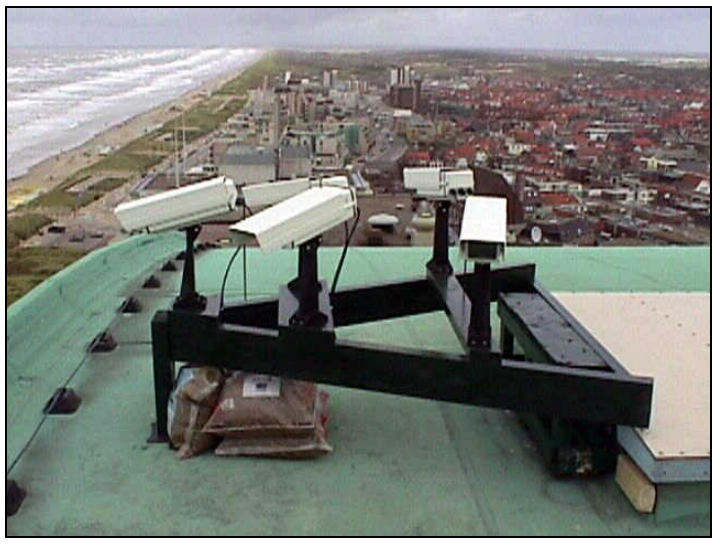

Figure 2-6 Argus station at the roof of hotel "Huis Ter Duin"

\subsection{Egmond experiment}

\subsection{Introduction}

The Egmond experiment was carried out in Egmond aan Zee in the period between October $14^{\text {th }}$ and November $3^{\text {rd }}, 2002$, as part of the EU-funded CoastView project. Measurements were done between October $23^{\text {rd }}$ and October $31^{\text {st }}$. Two different types of data were collected. First, a video collection of time stack data was designed and prepared for this period, using the existing methods developed in Oregon, USA. The video data set can be divided in three parts, because three different data collection schemes were used during the experiment. Besides video data, ground truth data were collected. Cross-shore and longshore current velocities were measured as well as pressure data and sediment concentration. The concentration and cross-shore velocity time series will not be used in this study. The wave conditions and the water levels during the measurement period are shown in Figure 2-7 to Figure 2-10. Wave angles are given in degrees from north with a clockwise positive direction. A large storm event took place on October $27^{\text {th }}$. This can be seen from the extreme wave heights at that date. Winds came mostly from the north-west. 


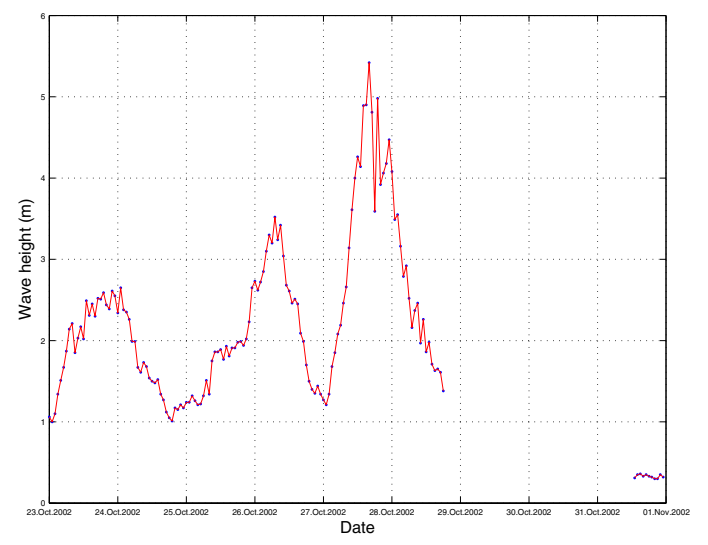

Figure 2-7 Wave height during the Egmond measurements

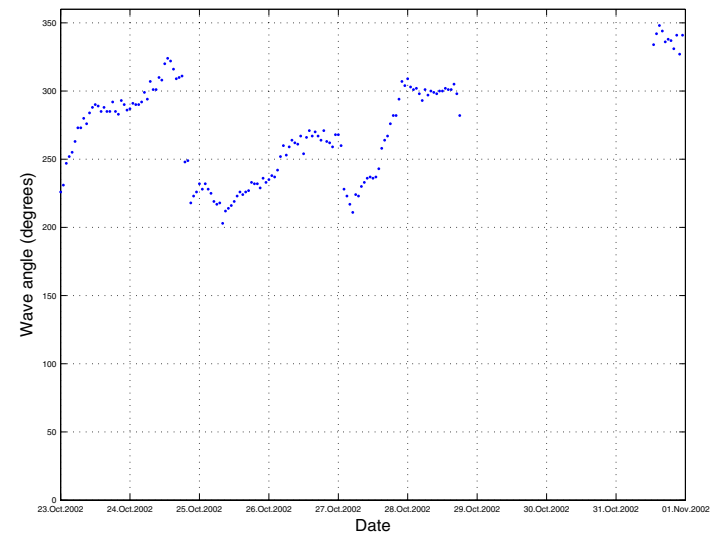

Figure 2-9 Wave angle during the Egmond measurements

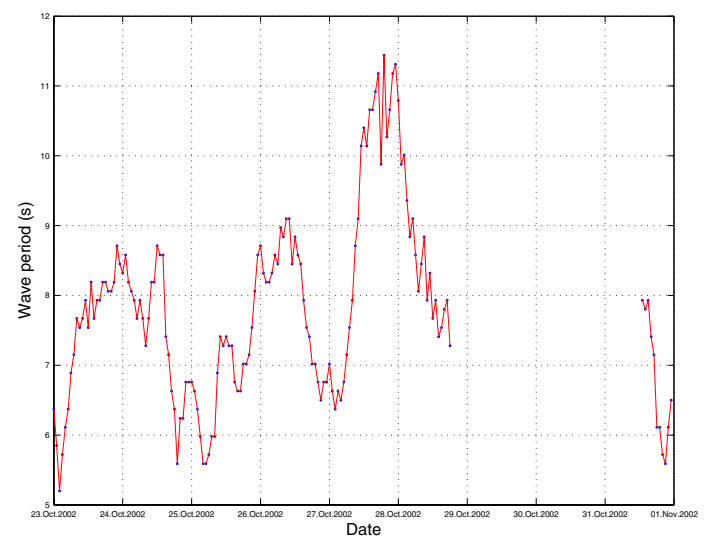

Figure 2-8 Wave period during the Egmond measurements

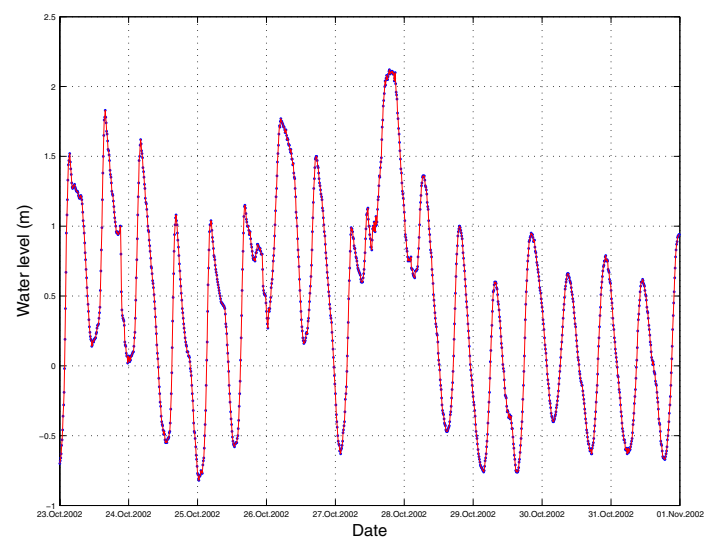

Figure 2-10 Water level during the Egmond measurements

\subsubsection{Egmond video data}

Video data collection files were designed using the Argus Pixel Toolbox from the Coastal Imaging Lab, Oregon State University (see Appendix D). In the design file three different array types have been defined, alpha arrays, cross-shore bathy arrays and longshore vbar arrays, which can be use for the quantification of wave characteristics, sub-tidal bathymetry and longshore currents respectively. This study is based on the analysis of vbar arrays. The collection was scheduled using the two north-looking cameras (1 and 2), for 2060 samples at 2 Hertz. A collection started 32 minutes past every hour and had a duration of 17.17 minutes. During night time no data collection was carried out, because of the lack of light necessary to obtain useful video data.

Three different data collection schemes have been used for the video data collection. The total measuring period can be divided in accordance with the use of these different schemes. 


\begin{tabular}{|l|l|}
\hline Part & Period \\
\hline 1 & $13 / 10 / 200217.32-17 / 10 / 200208.32$ \\
\hline 2 & $17 / 10 / 200209.32-22 / 10 / 200216.32$ \\
\hline 3 & $23 / 10 / 200206.32-03 / 11 / 200215.32$ \\
\hline
\end{tabular}

Table 2-1 Measuring period Egmond field experiment divided in accordance with three different data collection schemes

The different data collection schemes were used to adapt the designed data collection to the changing bathymetry. Furthermore a heuristic search was followed to optimise the amount of data that could be transferred to the Argus station. The layout of the pixel array design for these different parts of the video data set is shown in Figure 2-11 to Figure 2-16. The longshore vbar arrays are used in the longshore current velocity time stack analysis. The cross-shore bathy arrays can be used for analysis of the bathymetry via the wave celerity. The blue groups of pixels in the figures (alpha arrays) represent wave period and direction arrays. Only the longshore arrays will be used in the present study.

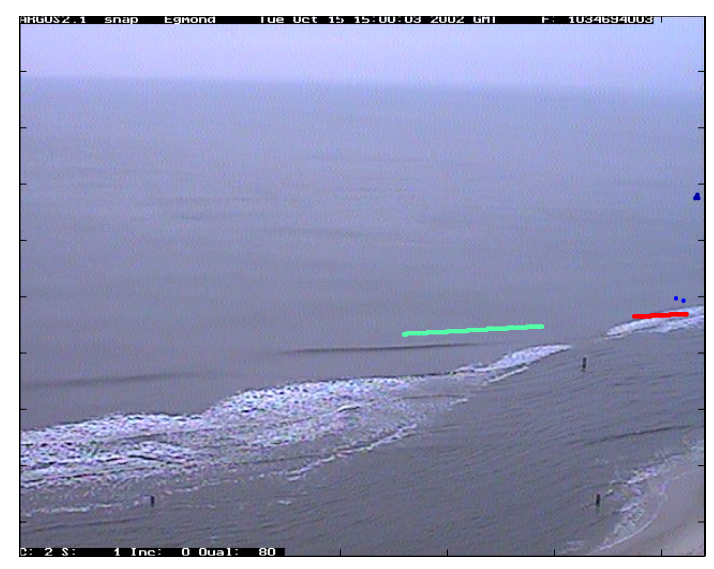

Figure 2-11 Pixel arrays in camera 2 - Part 1

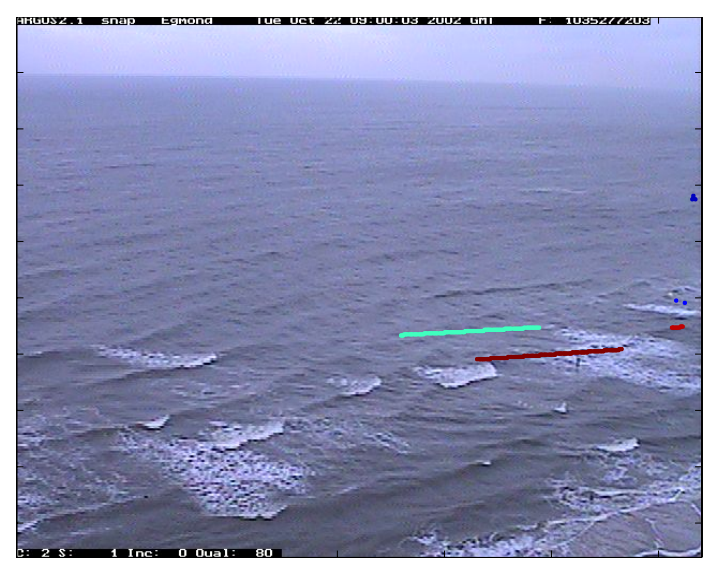

Figure 2-13 Pixel arrays in camera 2 - Part 2

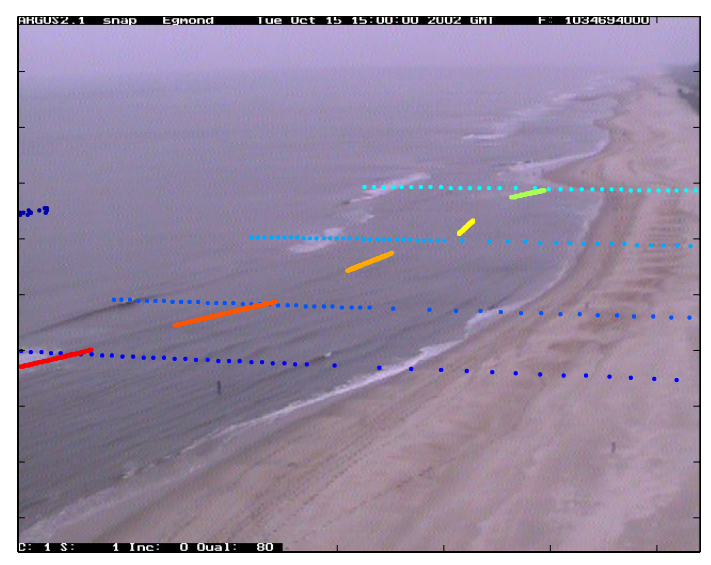

Figure 2-12 Pixel arrays in camera 1 - Part 1

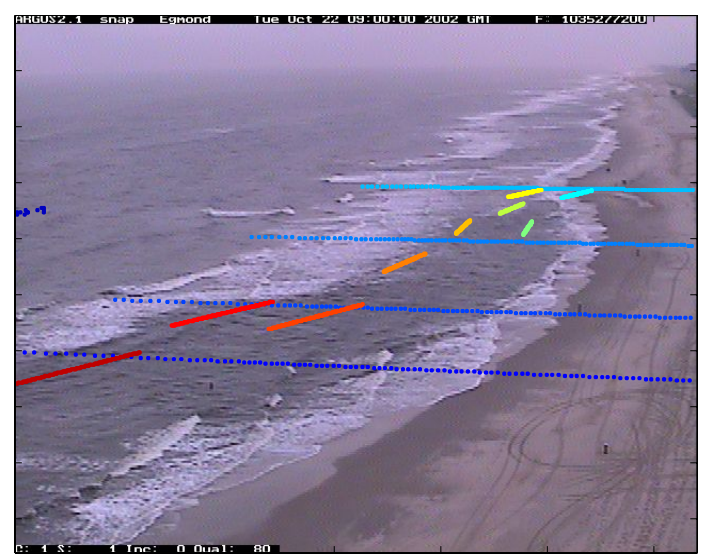

Figure 2-14 Pixel arrays in camera 1 - Part 2 


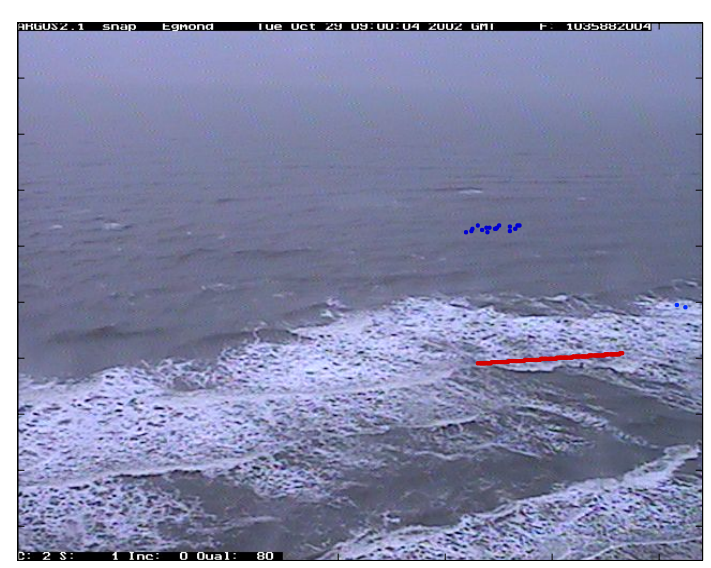

Figure 2-15 Pixel arrays in camera 2 - Part 3

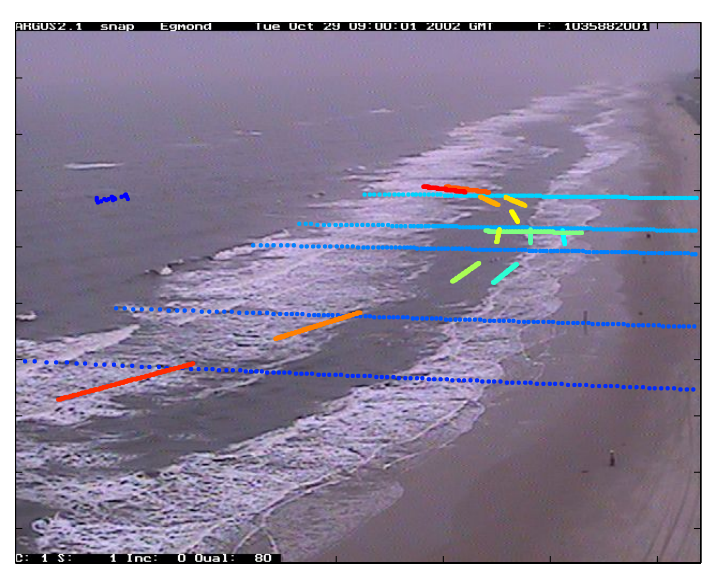

Figure 2-16 Pixel arrays in camera 1 - Part 3

\subsubsection{Egmond ground truth data}

During the field experiment, ground truth data were collected from October $19^{\text {th }}$ until October $31^{\text {st }}$. The measurements were carried out using four tripod stand alone frames. To each frame an electronics container was attached, which was connected to the measuring equipment. The measuring equipment consisted of an EMF (Electro Magnetic Flow) current velocity device, a pressure device and an OBS (Optical Back Scattering) device to measure sediment concentrations. Measurements were carried out in different configurations, depending on the bathymetry and the weather circumstances. For each frame the obtained data were split into the four different signals and calibrated using Hardhat software, a package developed by B. Grasmeijer at the University of Utrecht, which can split and calibrate data.

\subsubsection{Egmond data selection}

For further analysis a selection of the video and ground truth data is made. The first criterion for the selection is the simultaneity of video and ground truth data. All video data files which coincide with ground truth data files are selected and vice versa. The second criterion is the quality of the ground truth data files. A visual selection is made which filters out all the low quality time series from the ground truth data, meaning time series that were measured when the equipment was not entirely, or not at all, below the water surface any more. The last criterion is the sufficiency of light. Again, a visual selection is made, this time on the video data, filtering out all the video files that contain entirely black stacks, which indicates that the stack was collected at night. No collection was scheduled during nighttime, but the change in the duration of the night during the experiment caused the collection of some black stacks. No useful information can be drawn from this type of stack. The data selection which will be used for the analysis can be found in Appendix E. 


\subsection{Noordwijk experiment}

\subsection{Introduction}

Using the experience from the experiment that has been carried out in Egmond aan Zee in 2002, a second field experiment has been set up in Noordwijk aan Zee. This site was chosen because the measuring frames, used to obtain ground truth data, could be placed just in front of the Argus cameras, where the resolution of the video images is to 0.25 to 0.4 meters. The experiment at Noordwijk has been carried out from April $4^{\text {th }}$ until April $16^{\text {th }}$ in the year 2003. During this period, hourly time stack images have been collected at the Argus camera station as well as velocity time series from EMF measuring devices attached to tripod frames which were placed in the breaker zone. Measurements were carried out between April $7^{\text {th }}$ and April $16^{\text {th }}$. The wave conditions and the water levels during the measurement period are shown in Figure 2-17 to Figure 2-20. The period started with relatively large wave heights, which decreased in the course of the experiment, when the weather became calmer. Winds were mostly directed from north-west to north-east.

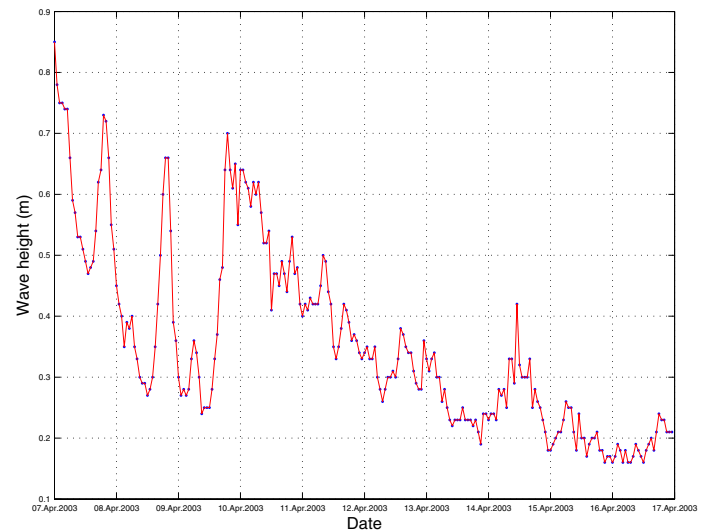

Figure 2-17 Wave height during the Noordwijk measurements

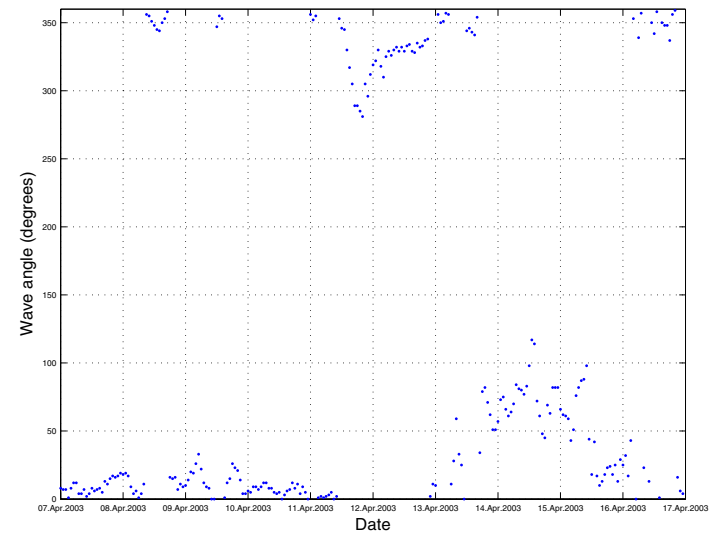

Figure 2-19 Wave angle during the Noordwijk measurements

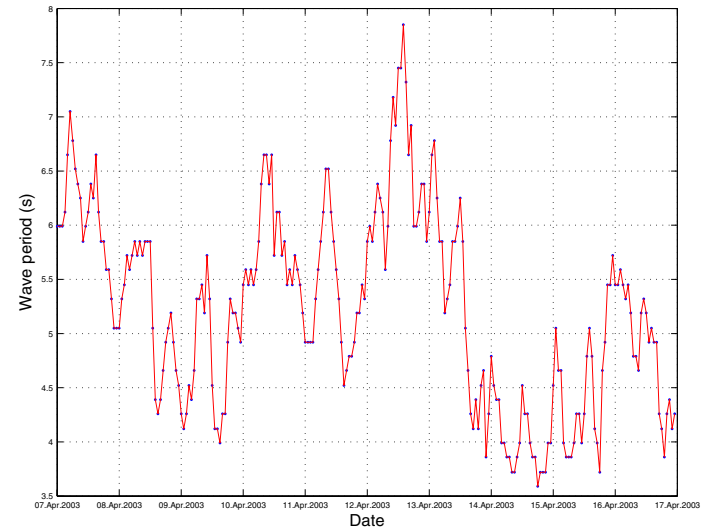

Figure 2-18 Wave period during the Noordwijk measurements

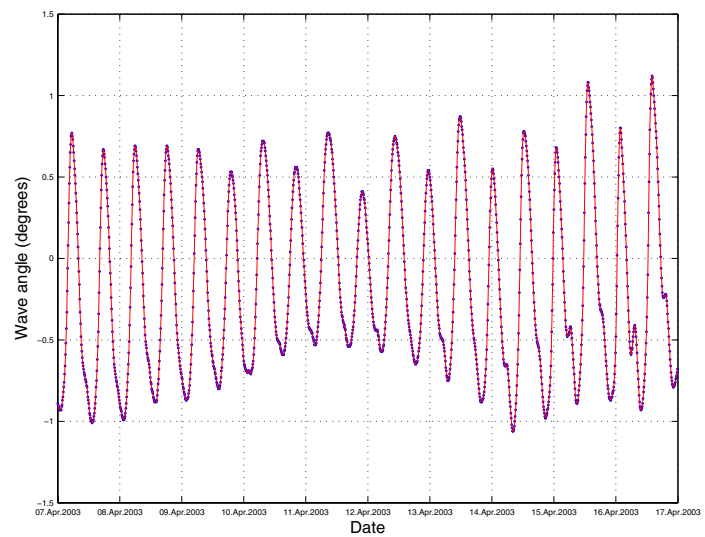

Figure 2-20 Water level during the Noordwijk measurements 


\subsubsection{Noordwijk video data}

Video data collection files were designed using the Argus Pixel Toolbox from the Coastal Imaging Lab, Oregon State University. A cross-shore series of nine parallel longshore pixel arrays was placed in the view of the middle camera, camera 4 . Five extra arrays were placed in the view of camera 5, in case problems would arise with camera 4 during the field experiment. Time stack data were collected hourly with a duration of 515 seconds with a frequency of 2 Hertz. The arrays in camera 4 have a length of 60 meters and a spacing between the collected pixels of 0.25 meters. This relatively large length and small pixel spacing are chosen in order to enable subsampling of smaller lengths or larger pixel spacings in the analysis phase. A length of 60 meters will be large enough to capture all existing foam patterns in an array. The spacing of 0.25 meters can be derived from the longshore pixel resolution at the location of the arrays, which amounts to 0.25 to 0.4 meters. The layout of the pixel array design is shown in Figure 2-21 and Figure 2-22. From April $4^{\text {th }}$ until April $7^{\text {th }}$ there was a problem with disk space at the Noordwijk Argus station. Due to this problem, the video data during this period have been lost. The disk space has been restored at April $7^{\text {th }} 12.00$ P.M.. From this time on the video data collection has functioned as it should.

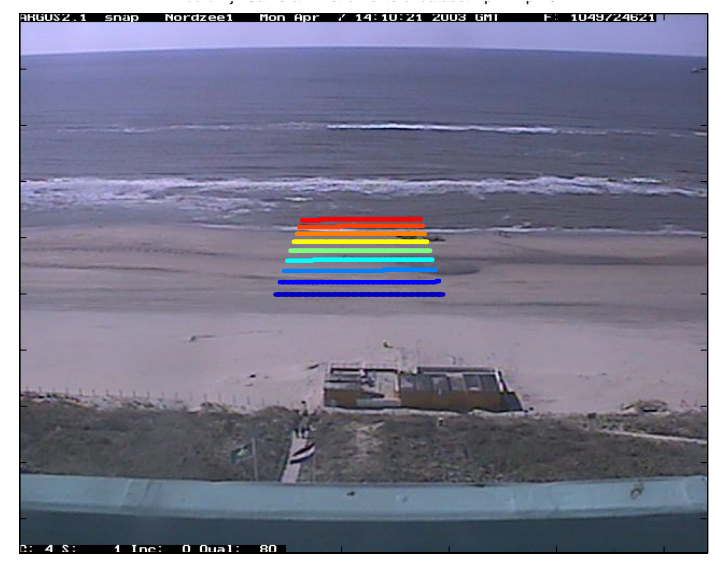

Figure 2-21 Pixel arrays in view of camera 4, Noordwijk aan Zee

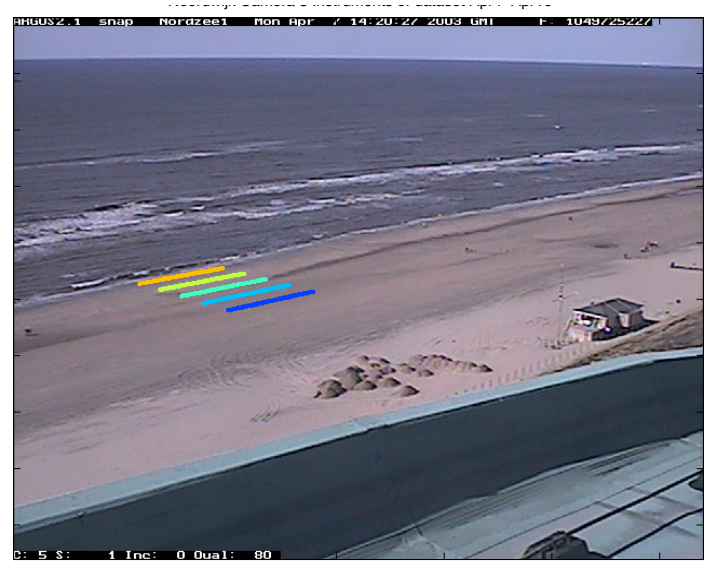

Figure 2-22 Pixel arrays in view of camera 5, Noordwijk aan Zee

\subsubsection{Noordwijk ground truth data}

During the field experiment, ground truth data were collected from April $4^{\text {th }}$ until April $16^{\text {th }}$. The measurements were carried out using two tripod stand alone frames. To each frame an electronics container was attached, which was connected to the measuring equipment. The equipment consisted of an EMF (Electro Magnetic Flow) current velocity measurement device and a pressure sensor. Measurements were carried out at different cross-shore positions, depending on the bathymetry, the tide and the weather circumstances. Due to a vandalistic incident on the beach on April $6^{\text {th }}$ the frames were slightly damaged. The main damage could be repaired on site. After a visual check of the measured data, it was decided to continue the measurements with the damaged measuring instruments. For each frame the obtained data were split into the four different signals (i.e. a longshore velocity, a crossshore velocity, a pressure and a concentration) and calibrated using Hardhat software. 


\subsubsection{Noordwijk data selection}

For further analysis a selection of the video and ground truth data is made, in the same way as is done with the Egmond data. The first criterion for the selection is the coincidence of video and ground truth data. The second criterion is the quality of the ground truth data files. The last criterion is the sufficiency of light. The data selection which will be used for the analysis can be found in Appendix E. 


\section{Model description}

\section{I Introduction}

In this research an alternative technique to 'measure' longshore current velocities is being investigated. This technique is called the Optical Current Meter (OCM) and has been developed by C.C. Chickadel at Oregon State University. The Optical Current Meter will be called the OCM in the remainder of this thesis. The input of the OCM consists of pixel intensity data $\mathrm{I}(\mathrm{t}, \mathrm{y} ; \mathrm{x})$ as described in section 1.2.2. The targets of the OCM are the foam patches that appear on the water surface after the waves have broken on the nearshore bar. These foam patches move slowly alongshore carried by the longshore current. The velocity of the foam patches therefore gives a measure of the longshore surface current velocity. In section 3.2, the OCM model is explained.

\subsection{The Optical Current Meter (OCM)}

The OCM transforms the video measurements of surface foam drift into an estimate of surface longshore current velocities through four signal processing steps: (1) computation of a two dimensional frequency-wavenumber spectrum, (2) transformation of the spectrum into a velocity-wavenumber spectrum, (3) integration through wavenumber to produce a velocity spectrum and finally (4) estimation of a single most representative velocity for that segment (Chickadel, 2002).

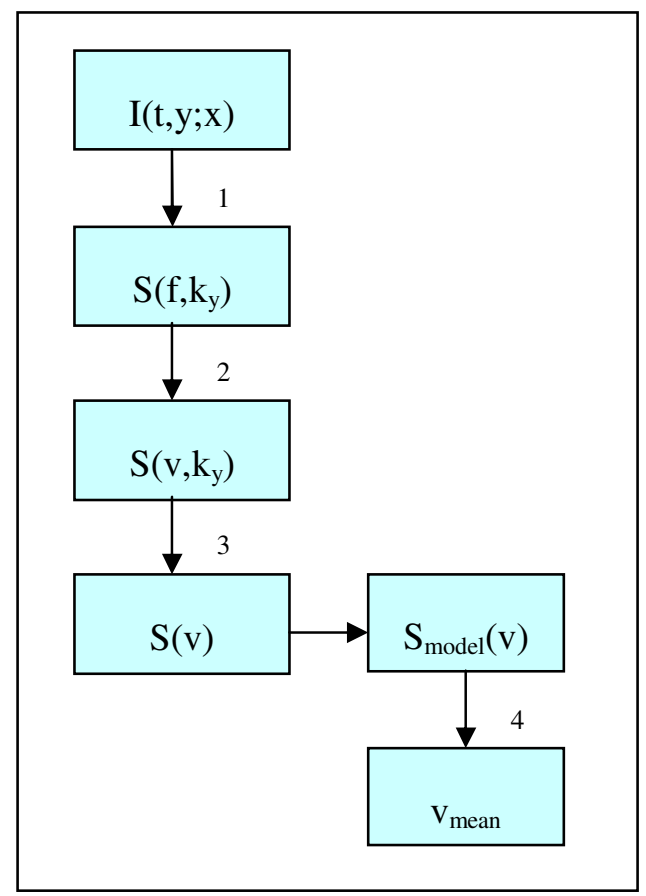

Figure 3-1 The OCM algoritm 


\section{$\mathbf{S}\left(\mathbf{f}, \mathbf{k}_{\mathbf{y}}\right)$ computation}

First, the pixel intensity data, $\mathrm{I}(\mathrm{t}, \mathrm{y} ; \mathrm{x})$, are transformed from a space $(\mathrm{y})$ and time (t) domain to a frequency-wavenumber domain using a two dimensional Fourier transform, resulting in $\hat{\mathrm{I}}\left(f, k_{y} ; x\right)$. The analytical form of this transformation is

$$
\hat{\mathrm{I}}\left(f, k_{y} ; x\right)=\iint B(t, y) I(t, y ; x) e^{-i 2 \pi f t} e^{-i 2 \pi k_{y} y} d t d y
$$

where $\mathrm{f}$ is the frequency and $\mathrm{k}_{\mathrm{y}}$ is the alongshore wavenumber (the reciprocal of the wavelength of the foam patches). The data are simultaneously windowed with a two dimensional Bartlett multiplicative filter, $\mathrm{B}(\mathrm{t}, \mathrm{y})$ to reduce smearing and leakage in the spectrum. The two-dimensional intensity variance spectrum, $\mathrm{S}\left(\mathrm{f}, \mathrm{k}_{\mathrm{y}}\right)$, is computed as

$$
S\left(f, k_{y}\right)=\hat{\mathrm{I}}\left(f, k_{y}\right) \hat{\mathrm{I}}\left(f, k_{y}\right) *
$$

where the asterisk $(*)$ denotes the complex conjugate.

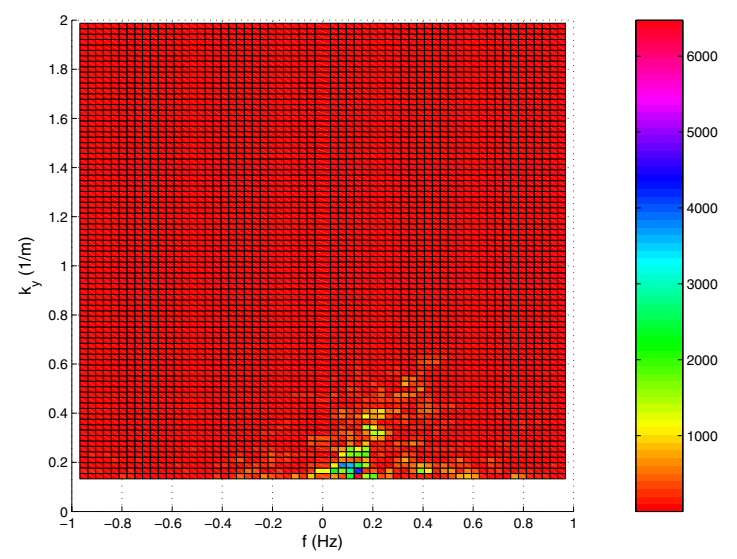

Figure 3-2 A frequency-wavenumber spectrum

\section{$\mathbf{S}\left(\mathbf{v}, \mathbf{k}_{\mathbf{y}}\right)$ transformation}

The spectrum is then transformed from frequency-wavenumber space to velocitywavenumber space. The relation

$$
v=\frac{f}{k_{y}}
$$

is used to transform the spectrum to the velocity-wavenumber space. To conserve variance in the transformation,

$$
\operatorname{var}\left\{S\left(f, k_{y}\right)\right\}=\iint S\left(f, k_{y}\right) d f d k_{y}=\iint S\left(v, k_{y}\right)\left|k_{y}\right| d v d k_{y}
$$


where $\left|\mathrm{k}_{\mathrm{y}}\right|$ is the Jacobian determinant and $\mathrm{S}\left(\mathrm{v}, \mathrm{k}_{\mathrm{y}}\right)$ is the velocity-wavenumber spectrum, so that

$$
S\left(v, k_{y}\right)=\frac{1}{\left|k_{y}\right|} S\left(f, k_{y}\right)
$$

To eliminate possible contamination resulting from obliquely incident waves which imitate rapidly moving sea foam, the velocity range is confined to $\pm 3 \mathrm{~m} / \mathrm{s}$. The alongshore projection of wave speed of an obliquely incident wave usually exceeds this range by far while longshore current velocities are typically within this range.

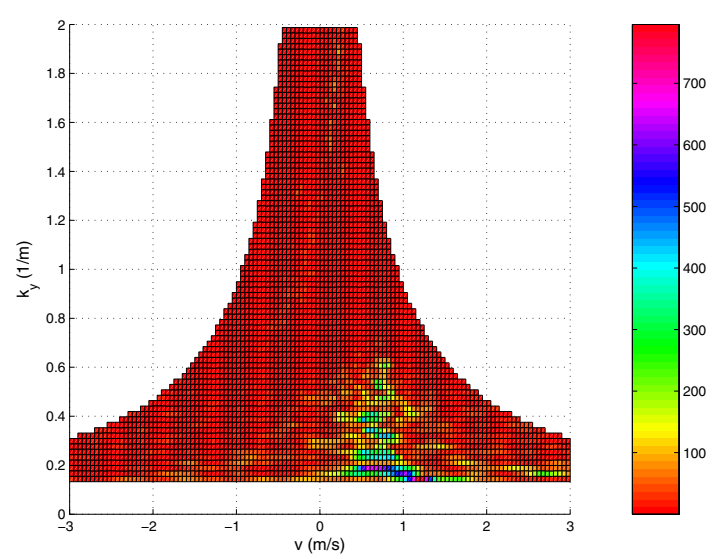

Figure 3-3 A velocity-wavenumber spectrum

\section{$S(v)$ transformation}

By integrating the $S\left(v, k_{y}\right)$ spectrum with respect to the wavenumber, the spectrum ${ }^{1}$ reduces to

$$
S(v)=\int_{k_{\min }}^{k_{n y q}} S\left(v, k_{y}\right) d k_{y}
$$

The leakage of inappropriate wave energy into $S(v)$ is minimised by excluding energy at wavenumbers below an arbitrary minimum wavenumber, $\mathrm{k}_{\min }$, chosen as $0.125 \mathrm{~m}^{-1}$. The upper limit of the integral is

$$
k_{n y q}=\frac{1}{2 \Delta y}
$$

\footnotetext{
${ }^{1}$ The expression 'spectrum' is questionable in this context, because $S(v)$ does not represent a range of velocities in relation with a frequency, but only a distribution of present velocities in a time window. An alternative expression, such as 'distribution', might be more appropriate. (pers. comm. Prof. dr. ir. Battjes)
} 
where $\mathrm{k}_{\mathrm{nyq}}$ is the Nyquist wavenumber and $\Delta \mathrm{y}$ is the sampling spacing. The resulting spectrum typically has a relatively large peak of energy representing the foam streaks and a background energy pattern due to video noise and low frequency intensity patterns.

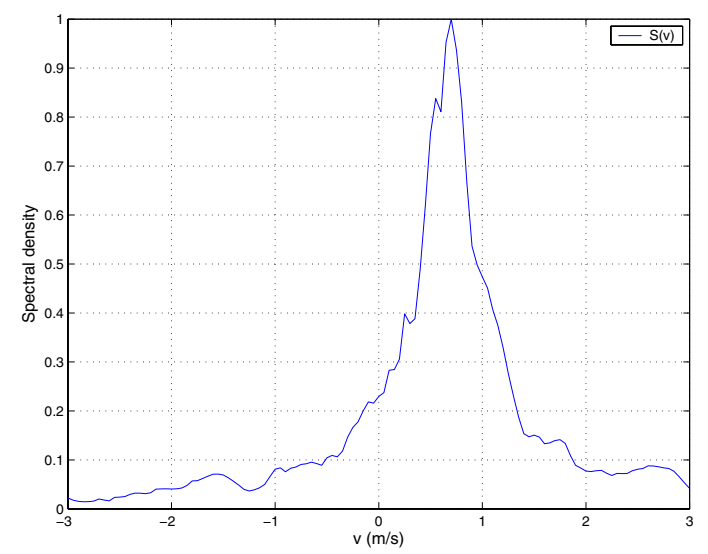

Figure 3-4 A velocity spectrum

\section{Velocity estimation}

Because the $S(v)$ spectrum is complicated with energy from the foam patches we would like to identify and background video noise, it is not trivial to determine a single surface velocity estimate. Chickadel(2002) uses a non-linear least-squares routine to fit a model of the velocity spectrum, $S_{\mathrm{m}}(\mathrm{v})$, to the observed spectrum, $\mathrm{S}(\mathrm{v})$. The model includes two components, the influence of the foam patches and that of the background noise.

$$
S_{m}(v)=S_{\text {foam }}(v)+S_{\text {noise }}(v)
$$

The spectral foam patch variance density is modelled as a Gaussian curve

$$
S_{\text {foam }}(v)=A_{\text {foam }} \exp \left[-\frac{(v-\bar{v})^{2}}{2 \sigma_{\text {foam }}^{2}}\right]
$$

where $\mathrm{A}_{\text {foam }}$ is the peak value, $\bar{v}$ is the mean longshore current velocity and $\sigma_{\text {foam }}$ is the standard deviation of the longshore current velocity in the time window. The background noise is derived by assuming an input white noise time series, with energy distributed uniformly over $\mathrm{S}\left(\mathrm{f}, \mathrm{k}_{\mathrm{y}}\right)$. Transforming this white noise to the velocity spectrum yields

$$
S_{n o i s e}(v)=\left\{\begin{array}{l}
A_{n o i s e} \frac{k_{n y q}^{2}}{2} \text { for }|v| \leq \frac{f_{n y q}}{k_{n y q}} \\
A_{n o i s e} \frac{f_{n y q}^{2}}{2 v^{2}} \text { for }|v|>\frac{f_{n y q}}{k_{n y q}}
\end{array}\right.
$$

where $A_{\text {noise }}$ is the noise peak factor, $\mathrm{f}_{\text {nyq }}$ is the sampling dependent Nyquist frequency and $\mathrm{k}_{\mathrm{nyq}}$ is the sampling dependent Nyquist wavenumber. The model then depends on the four 
parameters $\mathrm{A}_{\text {foam }}, \bar{v}, \sigma_{\text {foam }}$ and $\mathrm{A}_{\text {noise. }}$. The fitting routine uses the Gauss-Newton method to iterate to a best-fit solution in the least squares sense. A merit function, $M(\beta)$, is established which measures the sum of the squared differences between the model and the data

$$
M(\beta)=\sum_{i=1}^{N}\left[S(v(i))-S_{m}(v(i) ; \beta)\right]^{2}
$$

where $\beta$ represents the parameter space for the model. A search procedure is used such that a minimum of the merit function is found, using a non linear routine that iterates along the steepest descent of the merit function towards a solution. A best-fit solution, $\hat{\beta}$, is determined when the merit function is near the minimum and stops changing, or changing less than a pre-set tolerance level.

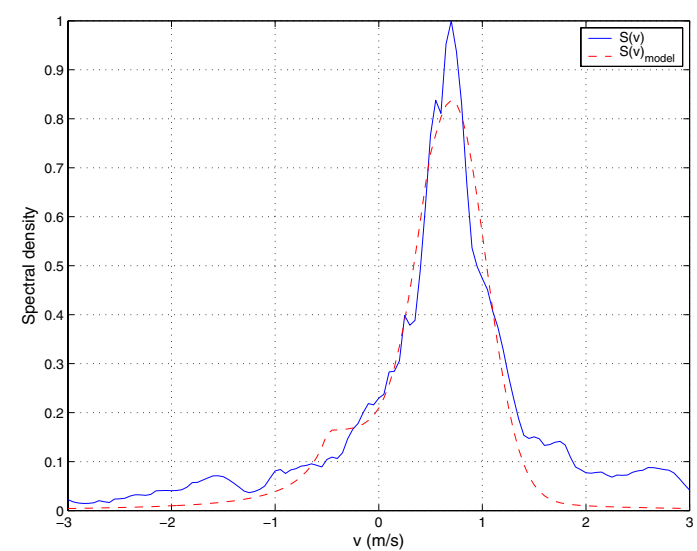

Figure 3-5 An observed velocity spectrum, together with the model of the velocity spectrum

\section{Velocity estimate quality}

The quality of the velocity estimate derived from the OCM algorithm is assessed by computing statistical measures of the 'goodness-of-fit' of the model and the best-fit parameters to the data. Additionally, because this method is based on the presence of foam due to wave breaking, a method was devised to provide an objective filter to reject estimates based on non breaking images or images with insufficient foam.

The condition of the model fit to the data is described using a $\chi^{2}$ statistic. This value is related to the merit function formulated in equation (3.11).

$$
\chi^{2}=\sum_{i=1}^{N}\left[\frac{S(v(i))-S_{m}(v(i) ; \hat{\beta})}{\sigma(i)}\right]^{2}
$$

where $\sigma(i)$ is the standard deviation of the measurement error at each point i. In this case, the measurement error is not known a priori. It is chosen to assign $\sigma(i)$ as a constant for all i, i.e. $10 \%$ of the maximum value of $\mathrm{S}(\mathrm{v})$, which allows for an objective measure of the 
statistical significance of the model fit regardless of total energy. From the $\chi^{2}$ statistic a significance level of overall fit can be found, the probability of the model fit P (Jenkins \& Watts, 1968).

Confidence intervals on the parameters, $\hat{\beta}$, in the model are estimated using a gradient of the parameter space as determined from the nonlinear fitting routine. A quadratic form is fitted to the gradient of the parameter space in the vicinity of the "best-fit" parameters to the data, which ideally lie at the global minimum of $M(\beta)$. The confidence intervals are estimated as the range of the parameters around $\hat{\beta}$, based on the quadratic form of the parameter space gradient, which give the best-fit parameters a 95\% probability (likelihood) given the data. A small 95\% confidence interval thus means a good quality of the "best-fit" parameter.

A proxy for the degree of breaking and residual foam in the video record is calculated, i.e. $I_{\text {range }}$, based on the intensity histogram of a time window of the time stack

$$
I_{\text {range }}=I_{95}-I_{50}
$$

where $I_{95}$ and $I_{50}$ are the $95^{\text {th }}$ and $50^{\text {th }}$ percentile intensity values, respectively. As the value of $I_{\text {range }}$ increases, so does the contrast and the degree of wave breaking and residual foam seen in the time stack.

\section{Time series generation}

A time series of longshore current velocity, $\mathrm{v}$, is estimated by applying the OCM in a stepwise fashion through the video, so that the window of analysis overlaps the previous window by some amount of time. The duration of the analysis window, $\mathrm{T}_{\mathrm{w}}$, and the time shift, $\mathrm{T}_{\mathrm{s}}$, are both unrestricted constants which have to be specified by the user. Previous research (Chickadel, 2002) has shown that, for the Duck site, a $\mathrm{T}_{\mathrm{w}}$ of 32 seconds has small errors while maintaining good resolution of low frequency motion.

\subsection{Model parameters}

So far, the pixel tools and the OCM have only been applied to data from the Duck site. Some parameters in the design tools and the sampling methods are chosen specifically for the Duck site and therefore do not necessarily need to have the same value when applied to a different location. The following parameters have values that have to be specified by the user:

- sampling spacing between points in an array, $\Delta y$

- duration of the analysis window, $\mathrm{T}_{\mathrm{w}}$

- time shift, $\mathrm{T}_{\mathrm{s}}$

- minimum wavenumber, $\mathrm{k}_{\min }$

- threshold for probability of the model fit, $\mathrm{P}$

- threshold for the confidence interval of the mean velocity, C.I. 
- threshold for the intensity range, $\mathrm{I}_{\text {range }}$

The Duck-settings of these parameters are shown in Table 3-1. The optimal values of $P$, $\mathrm{CI}_{\text {span }}$ and $\mathrm{I}_{\text {range }}$ at Duck can not be found in the literature (Chickadel, 2002). For these parameters values are assumed that have little to none influence on the model results.

\begin{tabular}{|l|l|l|}
\hline Parameter & Description & Duck value \\
\hline$\Delta \mathrm{y}$ & Sampling spacing & $0.25 \mathrm{~m}$ \\
\hline $\mathrm{T}_{\mathrm{w}}$ & Time window & $32 \mathrm{~s}$ \\
\hline $\mathrm{T}_{\mathrm{s}}$ & Time shift & $16 \mathrm{~s}$ \\
\hline $\mathrm{k}_{\min }$ & Minimum wavenumber & $0.125 \mathrm{~m}^{-1}$ \\
\hline $\mathrm{P}$ & Probability of the model fit & 0 \\
\hline $\mathrm{CI}_{\text {span }}$ & Confidence interval of $\mathrm{V}_{\text {mean }}$ & $4 \mathrm{~m} / \mathrm{s}$ \\
\hline $\mathrm{I}_{\text {range }}$ & Intensity range & 0 \\
\hline
\end{tabular}

Table 3-1 Values of the sampling and model parameters for the Duck site

In Chapter 5 the OCM will be calibrated and validated against the ground truth data described earlier for application at the Holland coast. 


\section{Applicability of the OCM}

\section{I Introduction}

To investigate the applicability of the OCM model, the model is run with the Egmond and the Noordwijk data set with the same parameter settings the way it has been used with the Duck data set in the USA. On the basis of that, we can judge on the applicability of the OCM in its present state to sites other than the Duck site, in this case the two sites along the Holland coast, Egmond and Noordwijk.

\subsection{Results}

The Egmond stacks have a length of $512 \mathrm{~s}$. With a time shift of $16 \mathrm{~s}$ this results in 32 individual velocity estimates $v_{i}$ per time stack. The Noordwijk stacks have a length of $256 \mathrm{~s}$ with the same time shift, resulting in 16 individual velocity estimates $v_{i}$ per time stack. The results of the Egmond and Noordwijk runs are shown in Figure 4-1 to Figure 4-4. In Figure 4-1 and Figure 4-2, the resulting stack averaged velocity $V_{\text {video }}$ is plotted against time, together with the standard deviation of the individual velocity estimates with respect to the stack averaged velocity $V_{\text {video }}$. When looking at the Egmond run, it can be seen that small velocities are calculated which have large standard deviations. The velocities become larger in time. The standard deviations become larger with larger velocities. The velocities from the Noordwijk run fluctuate more than the Egmond velocities. The standard deviations fluctuate also and a number of velocity estimates have a small standard deviation, which might indicate a reliable velocity estimate.

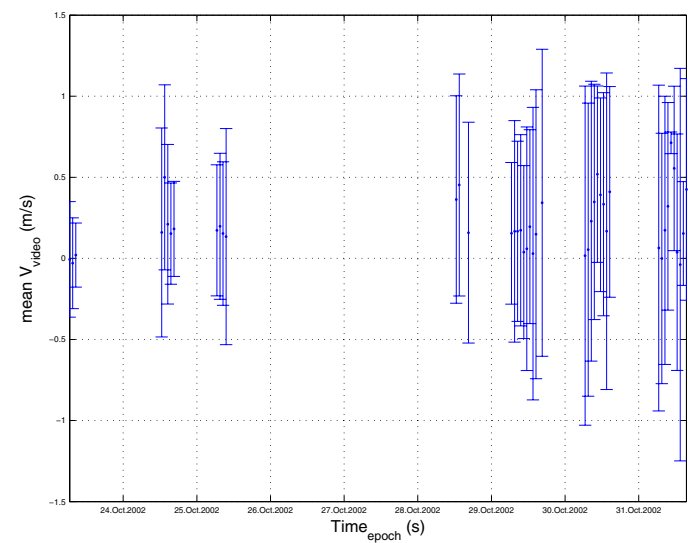

Figure 4-1 OCM velocity estimates together with their standard deviation plotted against time for the Egmond data set

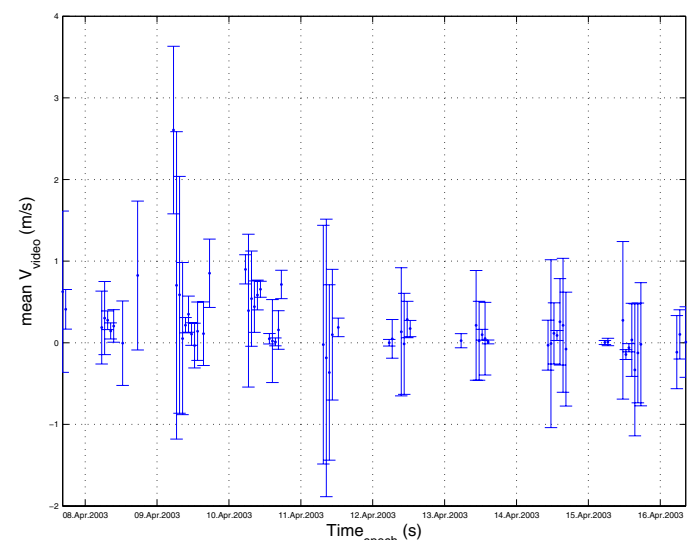

Figure 4-2 OCM velocity estimates together with their standard deviation plotted against time for the Noordwijk data set

Figure 4-3 and Figure 4-4 show the stack averaged velocity $V_{\text {video }}$ plotted against the mean of the simultaneously measured ground truth velocity time series. To quantify the quality of a set of stack averaged velocity estimates, four values are calculated, i.e. the root-meansquare value of the difference between $\mathrm{V}_{\text {video }}$ and the mean $\mathrm{V}_{\mathrm{gt}}\left(\Delta \mathrm{V}_{\mathrm{rms}}\right)$, the mean of that 
difference $\left(\Delta \mathrm{V}_{\text {mean }}\right)$, the standard deviation of that difference $\left(\sigma_{\Delta \mathrm{V}}\right)$ and the correlation coefficient between $\mathrm{V}_{\text {video }}$ and $\mathrm{V}_{\mathrm{gt}}(\gamma)$. The $\mathrm{i}$ in the following equations indicates the time stack number.

$$
\begin{gathered}
\Delta V=V_{\text {video }}-V_{g t} \\
\Delta V_{r m s}=\sqrt{\overline{(\Delta V)^{2}}} \\
\Delta V_{\text {mean }}=\frac{1}{n} \sum_{i=1}^{n} \Delta V_{i} \\
\sigma_{\Delta V}=\left(\frac{1}{n} \sum_{i=1}^{n}\left(\Delta V_{i}-\Delta V_{\text {mean }}\right)^{2}\right)^{\frac{1}{2}} \\
\gamma_{V_{\text {video }}, V_{g t}}=\frac{C\left(V_{\text {video }}, V_{g t}\right)}{\sqrt{C\left(V_{\text {video }}, V_{\text {video }}\right) C\left(V_{g t}, V_{g t}\right)}} \\
C=E\left(\left(V_{\text {video }}-\mu_{v_{\text {video }}}\right)\left(V_{g t}-\mu_{v_{g t}}\right)\right)
\end{gathered}
$$

The Egmond video data show no correlation with the ground truth data. A correlation coefficient of 0.03 is calculated for this set of data, which indicates no correlation at all. The Noordwijk data show a cloud of data points around zero and a small number of points along the 1:1 line, which is plotted in the same figure. Note that the scales of Figure 4-3 and Figure 4-4 are not equal. This is caused by an outlier in the Noordwijk dataset, for which a stack averaged velocity of $2.6 \mathrm{~m} / \mathrm{s}$ is estimated from the video data, while the ground truth velocity amounts to $0.05 \mathrm{~m} / \mathrm{s}$. In chapter 5 we will take a closer look at the accuracy of the velocity estimates from the video data. A correlation of 0.33 is found between the Noordwijk video and ground truth data (see Table 4-1). This is still a very weak correlation, but compared to the Egmond data it is quite good. The fact that the error values (the $\Delta$ values in Figure 4-3 and Figure 4-4) calculated for the Noordwijk data are larger than the error values from the Egmond data is caused by the outlier in the Noordwijk data. When leaving this point out of the analysis, the error values decrease and the correlation coefficient increases considerably, as is shown in Table 4-1. In the remainder of this study, the outlier will be included in the analysis, because the point satisfies the data selection criteria mentioned in section 2.4.4. 


\begin{tabular}{|l|l|l|l|l|}
\hline Site & $\Delta \mathrm{V}_{\text {rms }}(\mathrm{m} / \mathrm{s})$ & $\Delta \mathrm{V}_{\text {mean }}(\mathrm{m} / \mathrm{s})$ & $\sigma_{\Delta \mathrm{V}}(\mathrm{m} / \mathrm{s})$ & $\gamma(-)$ \\
\hline Egmond & 0.37 & 0.31 & 0.20 & 0.03 \\
\hline Noordwijk & 0.43 & 0.25 & 0.36 & 0.33 \\
\hline $\begin{array}{l}\text { Noordwijk } \\
\text { (without outlier) }\end{array}$ & 0.29 & 0.21 & 0.20 & 0.53 \\
\hline
\end{tabular}

Table 4-1 Quality values for the OCM model runs with the Egmond and the Noordwijk data set, using a Duckbased default setting for the model parameters

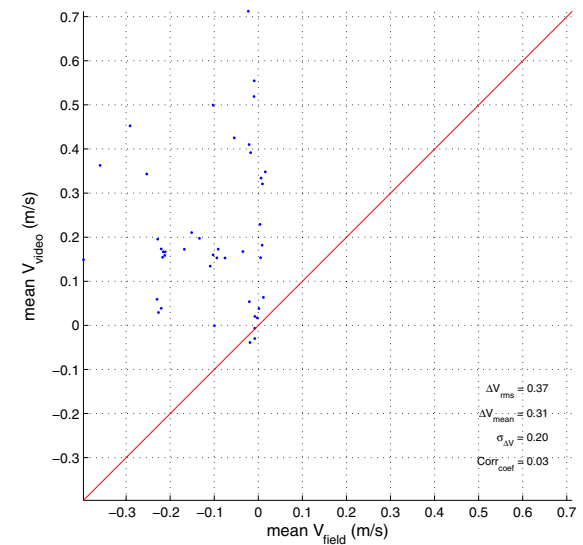

Figure 4-3 Mean $V_{\text {video }}$ plotted against mean $V_{g t}$ for the Egmond data set

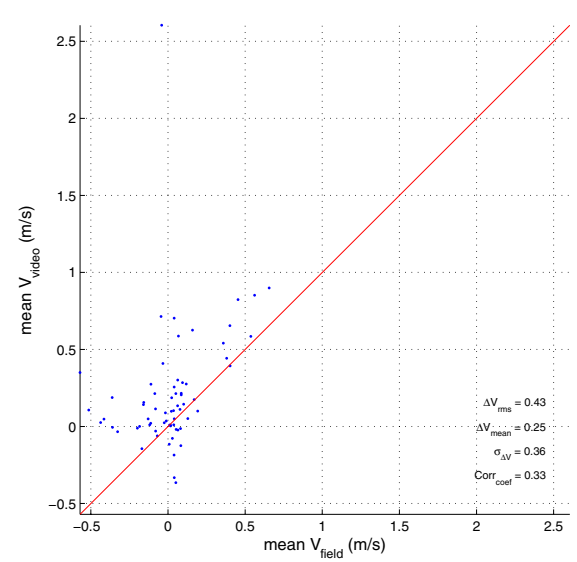

Figure 4-4 Mean $\mathrm{V}_{\text {video }}$ plotted against mean $\mathrm{V}_{\mathrm{gt}}$ for the Noordwijk data set

\subsection{Conclusions}

The results from the Egmond and the Noordwijk run show that the Duck-based OCM model is not directly applicable to the two sites at the Holland coast. The Egmond and Noordwijk sites have a wave climate that differs much from the wave climate in Duck, where the waves are long-crested and have a longer period than the waves along the Holland coast. The wave climate along the Holland coast is quite irregular and the waves are much shorter than in Duck. This may yield a more irregular flow pattern, hence a less-pronounced signal. This weaker signal to noise ratio may hamper appreciable model performance for some conditions. Still, there is a large difference between the Egmond and the Noordwijk results, even though the wave climate at both sites is approximately similar. The main difference between the two data sets is that the Egmond video data are collected from pixel arrays at a large distance from the Argus cameras. The longshore pixel dimension of the video images at that location is approximately $2 \mathrm{~m}$. The pixel arrays used to collect the Noordwijk video data were located just in front of the Argus cameras, where the longshore pixel dimension amounts to approximately 0.25 to $0.4 \mathrm{~m}$. The time stack images at Egmond show sharp bands in which the longshore intensity remains constant in longshore direction, while several points of the pixel array are defined in this band. This is caused by a pixel dimension of the video image that is larger than the pixel spacing of the pixel array $\Delta y$. A group of pixels in such a band thus records an equal intensity, which results in a spiky estimated velocity time series. The time stack images at Noordwijk do not show this problem and the estimated velocity time series show a smoother line. Because this is the only difference between the Egmond and the Noordwijk data set, it can be concluded that the difference in the results from the model runs with the two data sets is caused by the difference in the pixel 
dimension at the location where the data are collected from. The relation between the longshore pixel dimension and the sampling spacing in the pixel array $\Delta \mathrm{y}$ will be discussed in section 4.4.

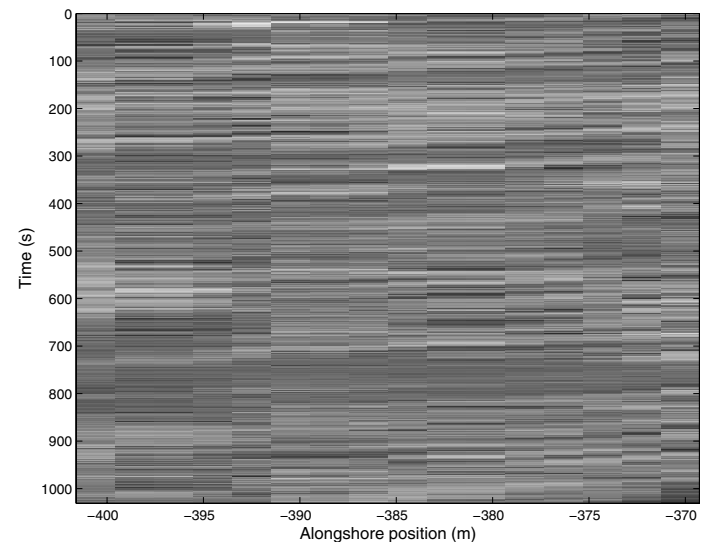

Figure 4-5 A time stack image at Egmond showing the intensity bands

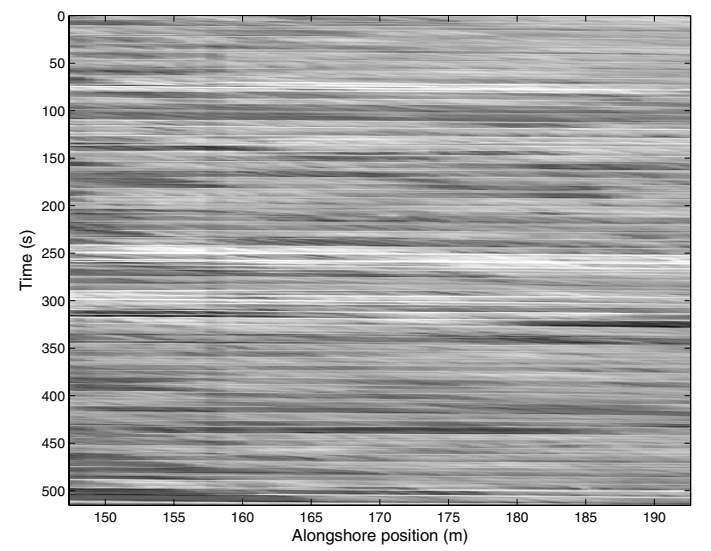

Figure 4-6 A time stack image at Noordwijk showing a smooth longshore intensity course

\subsection{Discussion on the sampling spacing}

As a result of the findings in section 4.3 it is necessary to analyse the value of the pixel spacing $\Delta y$ with regard to the longshore pixel dimension of the video image. First an analysis is carried out in which the OCM is run for five different values of the sampling spacing at one fixed location, i.e. an array in Noordwijk where the longshore pixel dimension is approximately $0.25 \mathrm{~m}$. The quality values per $\Delta \mathrm{y}$ value are shown in Table 4-2. Figure 4-7 to Figure 4-11 show the relation between the mean $V_{\text {video }}$ and the mean $V_{\text {ground }}$ truth for the selected data set for each tested value of the pixel spacing.

\begin{tabular}{|l|l|l|l|l|}
\hline Value $\Delta \mathrm{y}(\mathrm{m})$ & $\Delta \mathrm{V}_{\text {rms }}(\mathrm{m} / \mathrm{s})$ & $\Delta \mathrm{V}_{\text {mean }}(\mathrm{m} / \mathrm{s})$ & $\sigma_{\Delta \mathrm{V}}(\mathrm{m} / \mathrm{s})$ & $\gamma(-)$ \\
\hline 0.10 & 0.44 & 0.23 & 0.38 & 0.26 \\
\hline 0.25 & 0.43 & 0.25 & 0.36 & 0.33 \\
\hline 0.50 & 0.48 & 0.26 & 0.41 & 0.33 \\
\hline 0.75 & 0.50 & 0.26 & 0.43 & 0.29 \\
\hline 1.00 & 0.52 & 0.27 & 0.45 & 0.28 \\
\hline
\end{tabular}

Table 4-2 Quality values for different values of $\Delta y$ at Noordwijk

It can be seen from Table 4-2 that for pixel spacings larger than the longshore pixel dimension ( $0.25 \mathrm{~m}$ to $0.4 \mathrm{~m}$ in longshore direction), the error values become larger as well. The correlation coefficient becomes smaller in this case. The root-mean-square error has a minimum for a pixel spacing of $0.25 \mathrm{~m}$, which is close to the longshore resolution. The mean error continues to become smaller for smaller pixel spacings, but the variability of that error has a minimum for a pixel spacing of $0.25 \mathrm{~m}$, which is expressed in the standard deviation of the error. The correlation coefficient has a maximum at a pixel spacing of 0.25 and $0.50 \mathrm{~m}$. With a pixel spacing smaller than $0.25 \mathrm{~m}$, the correlation coefficient decreases considerably. The correlation coefficient decreases more gradually with pixel spacings larger than $0.50 \mathrm{~m}$. 


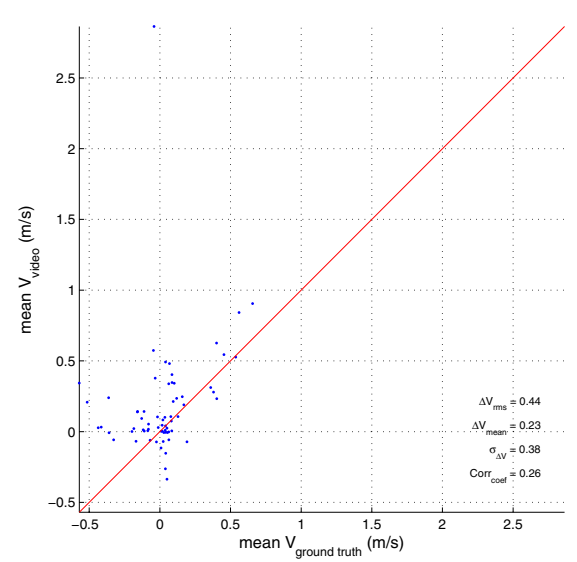

Figure 4-7 Mean $V_{\text {video }}$ plotted against mean $V_{g t}$ using a sampling spacing $\Delta \mathrm{y}=0.10 \mathrm{~m}$ at Noordwijk

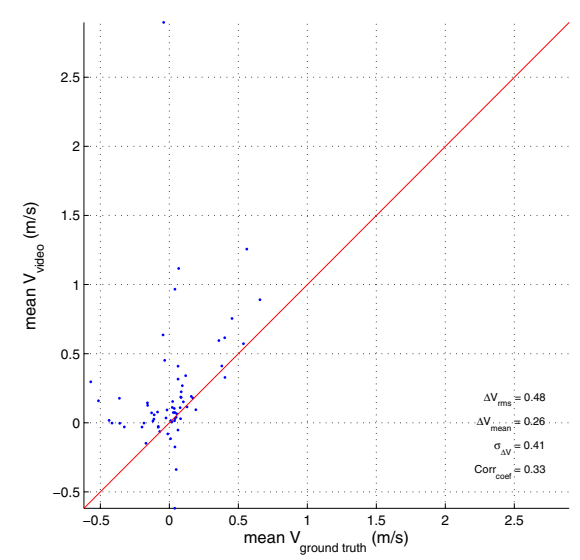

Figure 4-9 Mean $\mathrm{V}_{\text {video }}$ plotted against mean $\mathrm{V}_{\mathrm{gt}}$ using a sampling spacing $\Delta y=0.50 \mathrm{~m}$ at Noordwijk

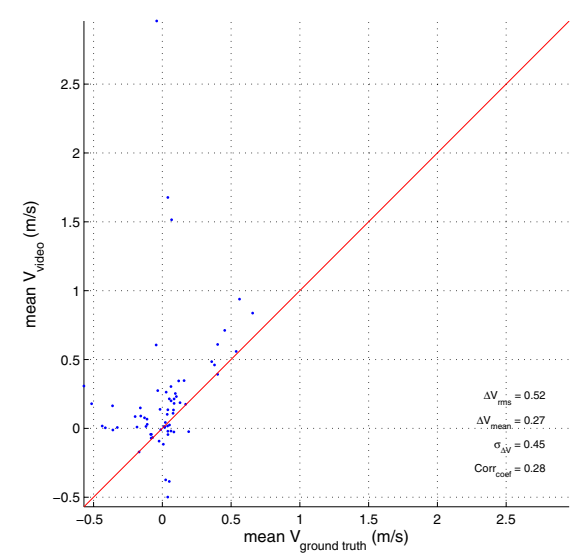

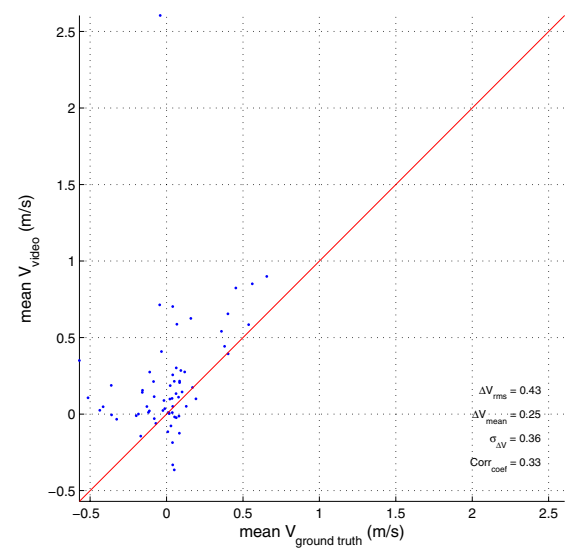

Figure 4-8 Mean $\mathrm{V}_{\text {video }}$ plotted against mean $\mathrm{V}_{\mathrm{gt}}$ using a sampling spacing $\Delta \mathrm{y}=0.25 \mathrm{~m}$ at Noordwijk

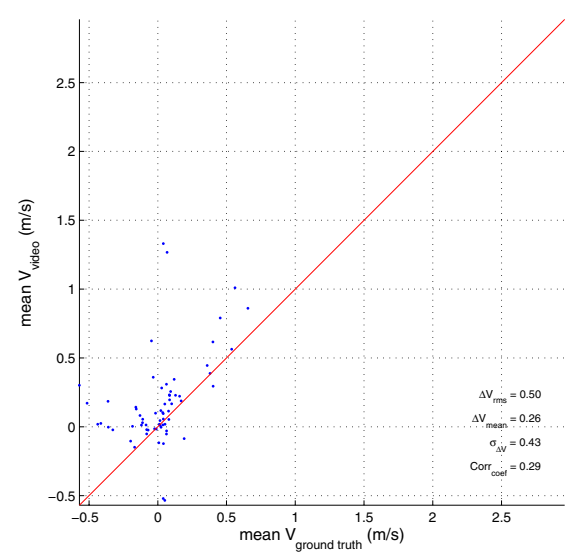

Figure 4-10 Mean $\mathrm{V}_{\text {video }}$ plotted against mean $\mathrm{V}_{\mathrm{gt}}$ using a sampling spacing $\Delta y=0.75 \mathrm{~m}$ at Noordwijk

Figure 4-11 Mean $\mathrm{V}_{\text {video }}$ plotted against mean $\mathrm{V}_{\mathrm{gt}}$ using a sampling spacing $\Delta \mathrm{y}=1.00 \mathrm{~m}$ at Noordwijk

It can thus be seen that arrays designed with a pixel spacing that has a value close to the longshore pixel dimension give the best results with the OCM. Arrays with a pixel spacing smaller than this pixel dimension do not gain additional information, because it is not possible to collect more pixels than the pixel dimension allows. Arrays with a pixel spacing larger than the longshore pixel dimension do not use the available data optimally, because 
less pixels are collected than available with respect to the longshore pixel resolution. For this reason it is recommended to use a pixel spacing $\Delta y$ in the order of the local dimension of a pixel footprint. In Noordwijk this pixel dimension is 0.25 to $0.4 \mathrm{~m}$, thus in the remainder of this study a pixel spacing of $0.25 \mathrm{~m}$ will be used with the Noordwijk data.

To verify the results of the pixel spacing analysis with regard to the longshore pixel dimension, an additional check is carried out using the Egmond data. The first analysis in section 4.2 has been carried out using a pixel spacing of $0.25 \mathrm{~m}$. This value is much smaller than the longshore pixel dimension at the location of the used pixel arrays, which amounts to approximately $2 \mathrm{~m}$. A run is carried out using a pixel spacing of $2 \mathrm{~m}$ with the Egmond data set. The results are shown in Table 4-3, Figure 4-12 and Figure 4-13.

\begin{tabular}{|l|l|l|l|l|}
\hline Value $\Delta \mathrm{y}(\mathrm{m})$ & $\Delta \mathrm{V}_{\text {rms }}(\mathrm{m} / \mathrm{s})$ & $\Delta \mathrm{V}_{\text {mean }}(\mathrm{m} / \mathrm{s})$ & $\sigma_{\Delta \mathrm{V}}(\mathrm{m} / \mathrm{s})$ & $\gamma(-)$ \\
\hline 0.25 & 0.44 & 0.23 & 0.38 & 0.26 \\
\hline 2.00 & 0.89 & 0.77 & 0.45 & 0.17 \\
\hline
\end{tabular}

Table 4-3 Quality values for different values of $\Delta y$ at Egmond

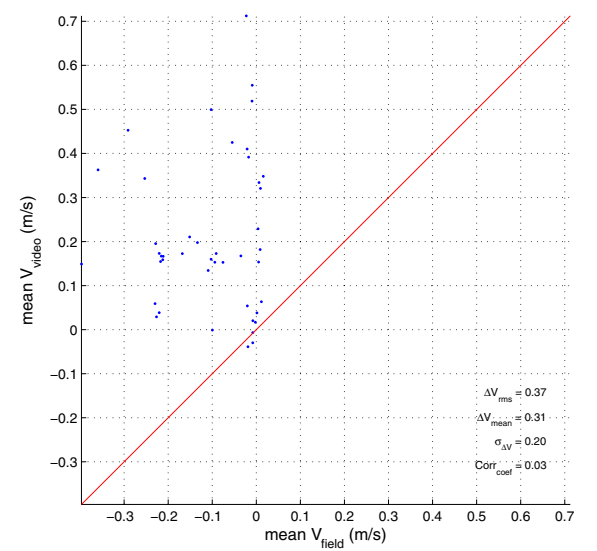

Figure 4-12 Mean $\mathrm{V}_{\text {video }}$ plotted against mean $\mathrm{V}_{\mathrm{gt}}$ using a sampling spacing $\Delta \mathrm{y}=0.25 \mathrm{~m}$ at Egmond

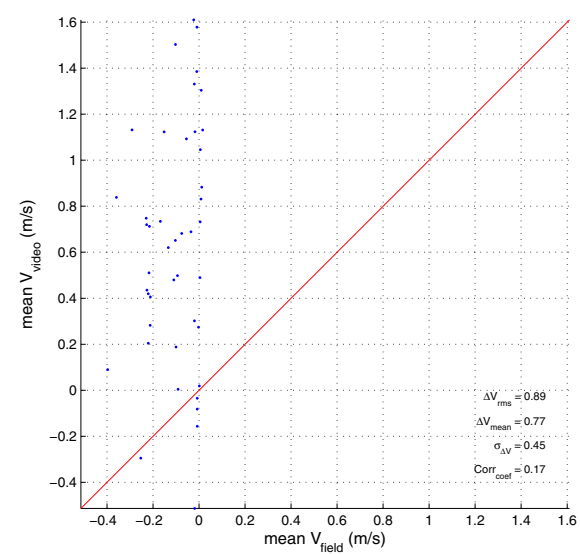

Figure 4-13 Mean $\mathrm{V}_{\text {video }}$ plotted against mean $\mathrm{V}_{\mathrm{gt}}$ using a sampling spacing $\Delta \mathrm{y}=2.00 \mathrm{~m}$ at Egmond

It is obvious from the error values that a sampling spacing of $2 \mathrm{~m}$ is not favourable for the quality of the velocity estimates by the OCM. The correlation coefficient is smaller with a sampling spacing of $2 \mathrm{~m}$ than with a sampling spacing of $0.25 \mathrm{~m}$. In this case a sampling spacing closer to the longshore pixel dimension does not improve the quality of the velocity estimate. The reason for this can be found in the fact that the pixel arrays in Egmond were located very far from the cameras, where the longshore pixel dimension is approximately 2 m.. Evidently this longshore pixel dimension is too large for the OCM to function in a proper way. Further research in this area is needed to get better insight in the range of the OCM and the corresponding longshore pixel dimensions. We have seen in section 4.2 that a longshore pixel dimension of $2 \mathrm{~m}$ is too large for the OCM to work in a proper manner. The un-calibrated OCM has shown to produce velocity estimates that have some correlation with ground truth data when arrays are used that are located at a place where the pixel dimension is $0.25 \mathrm{~m}$. Still, the correlation is very weak and it must be questioned if the OCM is suitable for application at the Holland coast. To answer this question, it is necessary to investigate the OCM parameter settings in better detail. In the remainder of this study we will only use 
the Noordwijk data set, because this set showed to provide at least some correlation with ground truth data when used in the OCM, as opposed to the Egmond data set.

\subsection{In summary}

The results from section 4.2 show that the Duck-based OCM model is not directly applicable to the two sites at the Holland coast. The difference in the results from the model runs with the two data sets is caused by the difference in the pixel dimension at the location of data collection. Arrays designed with a pixel spacing close to the longshore pixel dimension give the best results in the OCM. Arrays with a pixel spacing smaller than this pixel dimension do not gain additional information, because it is not possible to collect more pixels than the pixel dimension allows. Arrays with a pixel spacing larger than the longshore pixel dimension do not use the available data optimally, because less pixels are collected than available with respect to the longshore pixel resolution. It is thus recommended to use a pixel spacing $\Delta y$ in the order of the local dimension of a longshore pixel footprint. Furthermore, we are trying to recognize sine signals in pixel intensities due to foam patterns. Approximately 8 data points are needed to be able to pick up a sine signal. The high intensity signal of a foam patch embodies the positive half of the sine, for which 4 data points are needed. With a foam patch length of about 1 to $4 \mathrm{~m}$ along the Holland coast, we thus need a pixel spacing and thus a longshore pixel dimension with a maximum of 0.25 to 1 $\mathrm{m}$. The pixel arrays in Egmond were located very far from the cameras, where the longshore pixel dimension is approximately $2 \mathrm{~m}$. This longshore pixel dimension is too large for the OCM to function in a proper way. Further research in this area is needed to get better insight in the range of the OCM and the corresponding longshore pixel dimensions. 


\section{Accuracy of the OCM}

\section{I Introduction}

A calibration and validation of the OCM model at the Noordwijk site is carried out, to find the best setting of the model parameters mentioned in Chapter 3.3. Six different model parameters are examined to provide an accurate estimate of the longshore current velocity obtained from the OCM. These parameters include the duration of the time window, $\mathrm{T}_{\mathrm{w}}$, the time shift, $\mathrm{T}_{\mathrm{s}}$, the minimum wavenumber $\mathrm{k}_{\min }$, a probability threshold of the model fit, $\mathrm{P}, \mathrm{a}$ threshold of the $95 \%$ confidence interval of the model parameter $\mathrm{V}_{\text {mean }}, \mathrm{CI}_{\text {span }}$, and a contrast threshold value, $\mathrm{I}_{\text {range }}$. A sampling spacing of $0.25 \mathrm{~m}$ is used in the analysis, resulting from chapter 4.4 .

\subsection{Calibration and validation of the model parameters}

\subsection{Reference run}

Different values of each parameter will be tested, starting from a default setting for each parameter (see Table 5-1). The default values for the time window and the time shift are adopted from the Duck analysis. The default minimum wavenumber in the OCM has a value of $\mathrm{k}_{\min }=0.250 \mathrm{~m}^{-1}$ instead of the value used in the analysis of the Duck data set, $\mathrm{k}_{\min }=0.125$ $\mathrm{m}^{-1}$ because the presumed smaller foam patch size at the Dutch coast results in a smaller intensity wavelength in the time stack, thus a larger $\mathrm{k}_{\min }$ is expected to give better results. The optimal value of $\mathrm{k}_{\min }$ will be examined in the present chapter. The default value of the intensity range is chosen to be zero, so that no contrast threshold is applied during the analysis of the other parameters. The same applies to the probability of the model fit, for which a default value of zero is chosen too. The threshold of the $95 \%$ confidence interval of the model parameter $V_{\text {mean }}$ is set to a default value of $2 \mathrm{~m} / \mathrm{s}$ to each side of the mean, so that no influence of this threshold is present during analysis of the other parameters. Results from a run using the default values from Table 5-1, the reference run, are presented in Table 5-2 and Figure 5-1. The results from the different parameter tests will be compared to this reference.

\begin{tabular}{|l|l|l|}
\hline Parameter & Description & Default value \\
\hline$T_{w}$ & Time window & $32 \mathrm{~s}$ \\
\hline$T_{s}$ & Time shift & $16 \mathrm{~s}$ \\
\hline$k_{\min }$ & Minimum wavenumber & $0.25 \mathrm{~m}^{-1}$ \\
\hline$P$ & Probability of the model fit & 0 \\
\hline $\mathrm{CI}_{\text {span }}$ & Confidence interval of $\mathrm{V}_{\text {mean }}$ & $4 \mathrm{~m} / \mathrm{s}$ \\
\hline $\mathrm{I}_{\text {range }}$ & Intensity range & 0 \\
\hline
\end{tabular}

Table 5-1 Default setting of the model parameters 


\begin{tabular}{|l|l|l|l|l|}
\hline $\begin{array}{l}\text { Value quality control } \\
\text { parameters }\end{array}$ & $\Delta \mathrm{V}_{\mathrm{rms}}(\mathrm{m} / \mathrm{s})$ & $\Delta \mathrm{V}_{\text {mean }}(\mathrm{m} / \mathrm{s})$ & $\sigma_{\Delta \mathrm{V}}(\mathrm{m} / \mathrm{s})$ & Corr. Coeff. (-) \\
\hline Default & 0.25 & 0.18 & 0.18 & 0.52 \\
\hline
\end{tabular}

Table 5-2 Quality values of the reference run of model parameter analysis

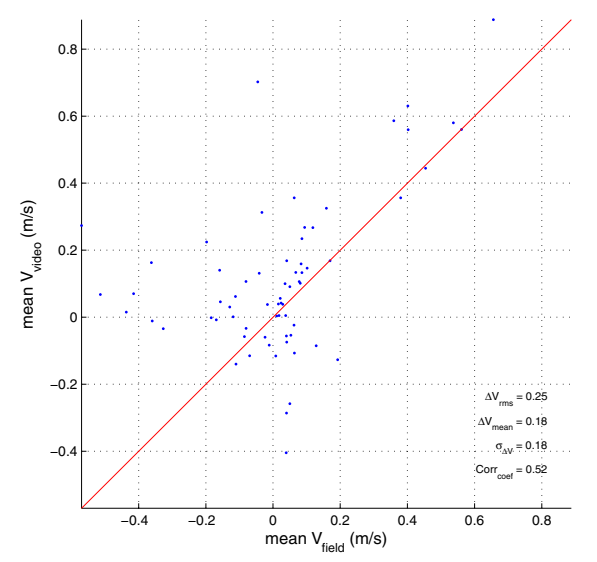

Figure 5-1 Mean $\mathrm{V}_{\text {video }}$ plotted against mean $\mathrm{V}_{\mathrm{gt}}$ for the reference run

\subsubsection{Time window and time shift}

The OCM steps through the time stack in time with a time shift $\mathrm{T}_{\mathrm{s}}$ and estimates a velocity for each time window $\mathrm{T}_{\mathrm{w}}$. In earlier research (Chickadel, 2002), the values of the optimal duration of the time window and time shift have been examined and values of $T_{w}=32 \mathrm{~s}$ and $T_{s}=16 \mathrm{~s}$ provided the best results for the Duck data set. Because the situation at the Dutch coast is different from that at Duck, a closer look is taken at the time window and time shift with which the data are handled by the OCM. The time window depends on the wave period of the breaking waves and on the duration of the existence of the foam patches on the water surface. Because the wave period along the Holland coast is much shorter than in Duck, it is expected that a shorter time window, and thus time shift, can be used while obtaining the same results from the OCM. We do not know anything about the persistency of the foam patches in Duck as compared to Noordwijk, so no predictions can be made on that part.

Five different values are tested both for $T_{w}$ and $T_{s}$. The two parameters are examined together, with a time shift half the duration of the time window, to obtain a good coverage of the data. The tested values are shown in Table 5-3, together with their error values and correlation coefficient. Figure 5-2 to Figure 5-6 show the relation between the mean $\mathrm{V}_{\text {video }}$ and the mean $\mathrm{V}_{\mathrm{gt}}$ for the selected data set for each tested value of $\mathrm{T}_{\mathrm{w}}$ and $\mathrm{T}_{\mathrm{s}}$. 


\begin{tabular}{|l|l|l|l|l|l|}
\hline Value $\mathrm{T}_{\mathrm{w}}(\mathrm{s})$ & Value $\mathrm{T}_{\mathrm{s}}(\mathrm{s})$ & $\Delta \mathrm{V}_{\text {rms }}(\mathrm{m} / \mathrm{s})$ & $\Delta \mathrm{V}_{\text {mean }}(\mathrm{m} / \mathrm{s})$ & $\sigma_{\Delta \mathrm{V}}(\mathrm{m} / \mathrm{s})$ & $\begin{array}{l}\text { Corr. Coeff. } \\
(-)\end{array}$ \\
\hline 8 & 4 & 0.27 & 0.19 & 0.19 & 0.47 \\
\hline 16 & 8 & 0.25 & 0.18 & 0.17 & 0.51 \\
\hline 32 (default) & 16 (default) & 0.25 & 0.18 & 0.18 & 0.52 \\
\hline 49 & 24.5 & 0.28 & 0.20 & 0.19 & 0.49 \\
\hline 64 & 32 & 0.30 & 0.23 & 0.19 & 0.44 \\
\hline
\end{tabular}

Table 5-3 Quality values for different values of $T_{w}$ and $T_{S}$

The differences in the error values and correlation coefficient between the five different time window and time step combinations are very small, as can be seen from Table 5-3. There appears to be a minimum of the error values around the time window-shift combinations of 16-8 and 32-16, but the difference with the other combinations is not convincing. The correlation coefficient has a maximum for a time window of $32 \mathrm{~s}$ and a time shift of $16 \mathrm{~s}$, but again the difference with the other time window-shift combinations is small.

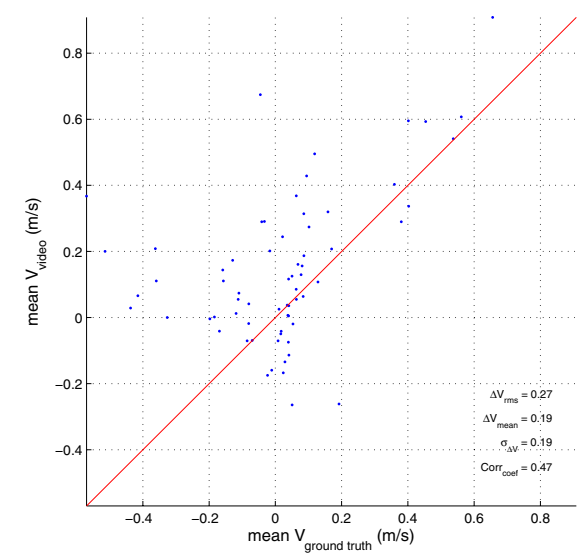

Figure 5-2 Mean $\mathrm{V}_{\text {video }}$ plotted against mean $\mathrm{V}_{\mathrm{gt}}$ with $\mathrm{T}_{\mathrm{W}}=8 \mathrm{~s}$ and $\mathrm{Ts}=4 \mathrm{~s}$

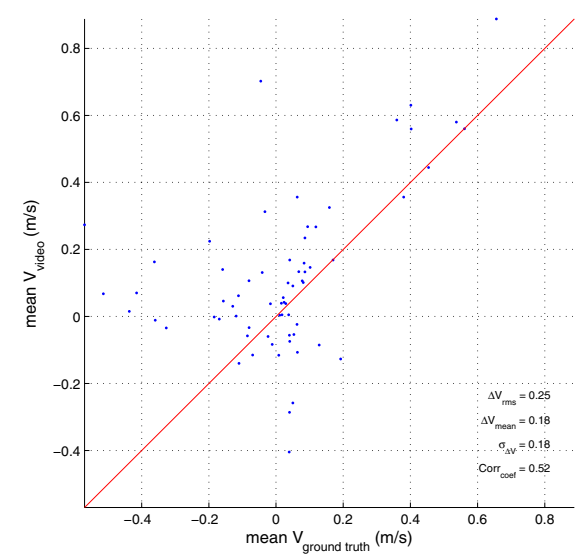

Figure 5-4 Mean $\mathrm{V}_{\text {video }}$ plotted against mean $\mathrm{V}_{\mathrm{gt}}$ with $\mathrm{T}_{\mathrm{W}}=32 \mathrm{~s}$ and $\mathrm{Ts}=16 \mathrm{~s}$

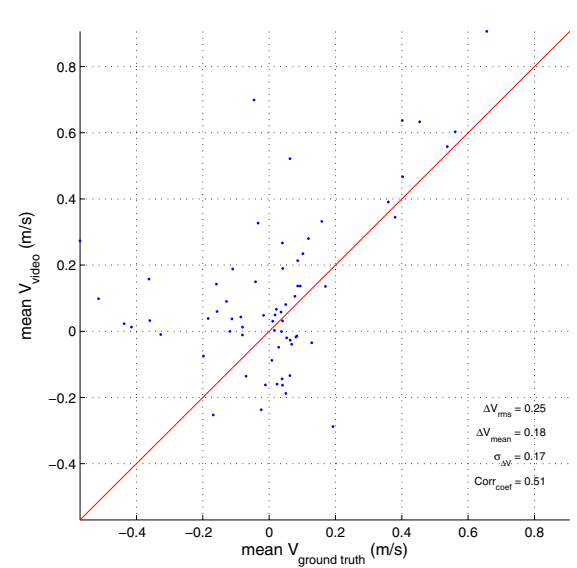

Figure 5-3 Mean $\mathrm{V}_{\text {video }}$ plotted against mean $\mathrm{V}_{\mathrm{gt}}$ with $\mathrm{T}_{\mathrm{W}}=16 \mathrm{~s}$ and $\mathrm{Ts}=8 \mathrm{~s}$

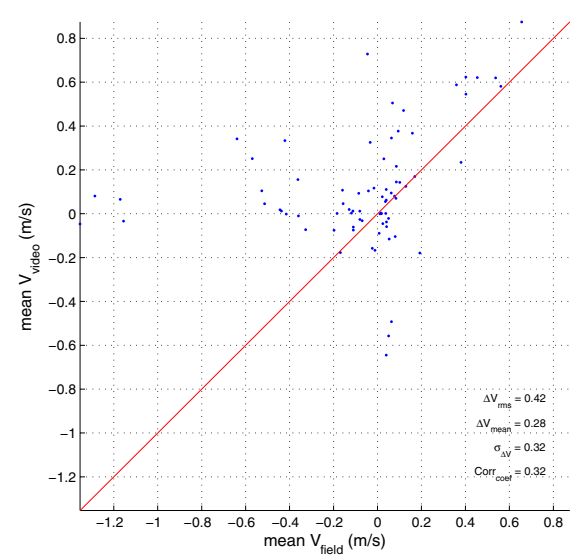

Figure 5-5 Mean $\mathrm{V}_{\text {video }}$ plotted against mean $\mathrm{V}_{\mathrm{gt}}$ with $\mathrm{T}_{\mathrm{W}}=49 \mathrm{~s}$ and $\mathrm{Ts}=24.5 \mathrm{~s}$ 


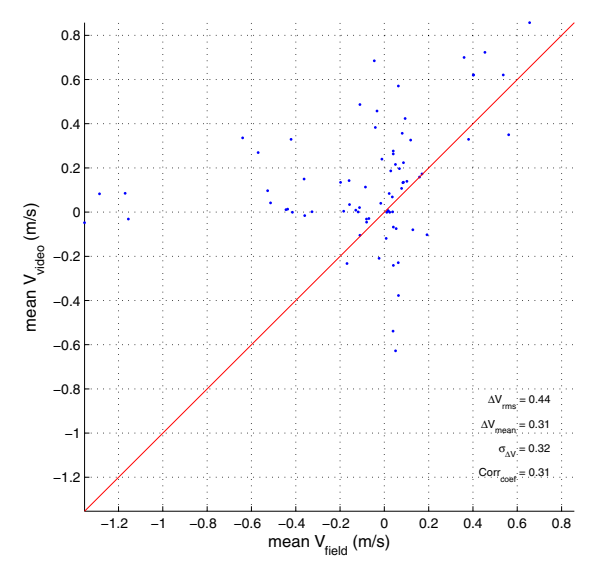

Figure 5-6 Mean $\mathrm{V}_{\text {video }}$ plotted against mean $\mathrm{V}_{\mathrm{gt}}$ with $\mathrm{T}_{\mathrm{W}}=64 \mathrm{~s}$ and $\mathrm{Ts}=32 \mathrm{~s}$

The OCM is virtually insensitive to changes in the time window and time shift with which it steps through the time stack in time. This may be caused by the fact that the weather conditions during the Noordwijk field experiment were calm and no long waves have occurred. It is advised to carry out another experiment when longer waves do occur, to be able to come to conclusions about the behaviour of the OCM with respect to changes of $T_{w}$ and $\mathrm{T}_{\mathrm{s}}$. With a smaller $\mathrm{T}_{\mathrm{w}}$ and $\mathrm{T}_{\mathrm{s}}$, the time stacks are shorter in time dimension while producing the same number of individual velocity estimates $v_{i}$ as a longer time stack with larger $T_{w}$ and $T_{s}$. The computer time per $v_{i}$ is smaller with a smaller $T_{w}$, so the total computer time per time stack is smaller too, while providing the same number of individual velocity estimates. When we have already collected time stacks with a constant length, we obtain more individual velocity estimates from a time stack when using a smaller $T_{w}$ and $T_{s}$. This might result in a more reliable estimate of the stack averaged velocity $\mathrm{V}_{\text {video. }}$. There is a limit to this reduction of $T_{w}$, because when we will choose $T_{w}$ too small, no useful signal can be picked up from the time stack. With the resources available at WLIDelft Hydraulics, the computer time is not of overriding importance. The accuracy of the velocity estimate and the file size of the stack (with respect to transport via phoneline) are. $\mathrm{A} \mathrm{T}_{\mathrm{w}}$ of $16 \mathrm{~s}$ and a $\mathrm{T}_{\mathrm{s}}$ of $8 \mathrm{~s}$ give the best results while being the most economical values, even if the difference with other values is very small. For future analysis, a $\mathrm{T}_{\mathrm{w}}$ of $16 \mathrm{~s}$ and a $\mathrm{T}_{\mathrm{s}}$ of $8 \mathrm{~s}$ are recommended for use with the OCM at the Argus sites along the Holland coast.

\subsubsection{Minimum wavenumber}

The OCM integrates the $S\left(v, k_{y}\right)$ spectrum with respect to the wavenumber $k_{y}$, to arrive at the $\mathrm{S}(\mathrm{v})$ spectrum (see chapter 3.2). The leakage of inappropriate wave energy into $\mathrm{S}(\mathrm{v})$ is minimised by excluding energy at wavenumbers below a minimum wavenumber, $\mathrm{k}_{\min }$. The value of this $\mathrm{k}_{\min }$ has in previous research been chosen arbitrarily (Chickadel, 2002). In the present paragraph an analysis is presented to investigate the behaviour of the OCM with respect to changes in the minimum wavenumber value.

A large minimum wavenumber means a small maximum wavelength of the foam patterns in the time stack. With $\mathrm{k}_{\min }=0.50 \mathrm{~m}^{-1}$, the maximum wavelength has a value of $2 \mathrm{~m}\left(\mathrm{k}_{\min }=1 / \mathrm{L}\right)$. A foam patch has a high intensity. The wavelength of a foam pattern has a value twice as large as the size of the foam patch, which is only the crest of the intensity wave. An intensity 
wavelength of $2 \mathrm{~m}$ thus means a maximum foam patch size of only $1 \mathrm{~m}$. At the Noordwijk site, an average longshore foam patch size of about 1 to $4 \mathrm{~m}$ can be observed from the time stack images. A $\mathrm{k}_{\min }$ of $0.50 \mathrm{~m}^{-1}$ will thus neglect a large amount of useful data. This can also be seen when we look at plots of $S(f, k)$ for different values of $k_{\min }$ and the resulting $S(v)$ and $\mathrm{S}(\mathrm{v})_{\text {model }}$ (see Figure 5-7 to Figure 5-14). The spectra are normalised with respect to the maximum spectral density of the observed spectra. The minimum wavenumber is the only model parameter in the calibration that affects the spectrum. When the frequencywavenumber spectrum is cut off at a low wavenumber, for instance $k_{\min }=0.0625 \mathrm{~m}^{-1}$, the intensities that play a role in the spectrum are generated by breaking wave crests that pass the pixel array and that have a large intensity wavelength (small wavenumber). The horizontal intensity streak in Figure 5-7 represents these breaking wave crests. Compared to these long crested and fast moving intensity waves, the longshore moving foam patterns do not play a role in the spectrum. As mentioned before, the length of the longshore foam patterns along the Holland coast are in a range of 1 to 4 meters, thus the wavelength varies between 2 and 8 meters. This corresponds to a wavenumber in the range of 0.125 to $0.5 \mathrm{~m}^{-1}$. This can be seen from Figure 5-9, where a high spectral density in the normalized spectrum exists in that range of $\mathrm{k}_{\min }$. The resulting $\mathrm{S}(\mathrm{v})$ spectrum shows a well pronounced peak at $\mathrm{v}_{\text {mean }}=0.57 \mathrm{~m} / \mathrm{s}$. When we look at the spectra with a $\mathrm{k}_{\min }$ of $0.25 \mathrm{~m}^{-1}$, the peak in $\mathrm{S}(\mathrm{v})$ is still as well pronounced as in the $\mathrm{k}_{\min }=0.125 \mathrm{~m}^{-1}$ spectrum. When increasing the minimum wavenumber further to $0.5 \mathrm{~m}^{-1}$, the $\mathrm{S}(\mathrm{v})$ spectrum starts to show additional peaks, besides the pronounced peak of the longshore moving foam patches. These peaks are caused by background noise, that can be seen in Figure 5-13 as the high spectral density spots in the wavenumber range of 1 to $2 \mathrm{~m}^{-1}$. A further increase of the minimum wavenumber to $0.75 \mathrm{~m}^{-1}$ cuts off all the influence of the longshore moving foam patterns that are useful for the analysis and only shows the influence of the background noise. The peak in the S(v) spectrum is moved to a value of $\mathrm{v}_{\text {mean }}=-0.016 \mathrm{~m} / \mathrm{s}$, representing the mean velocity of the background noise.

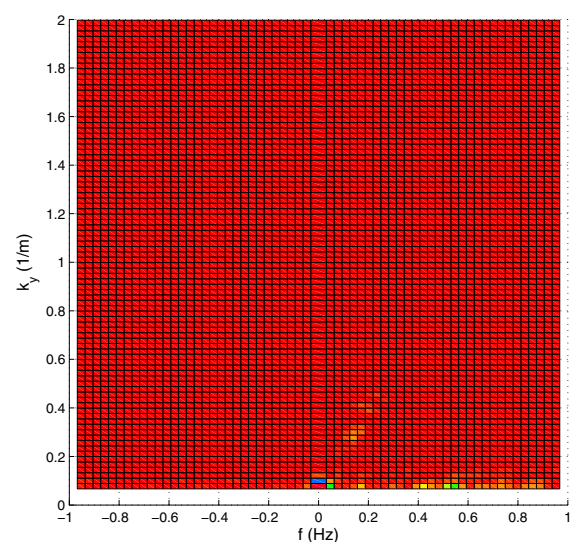

Figure 5-7 $\mathrm{S}(\mathrm{f}, \mathrm{k})$ with $\mathrm{k}_{\min }=0.0625 \mathrm{~m}^{-1}$

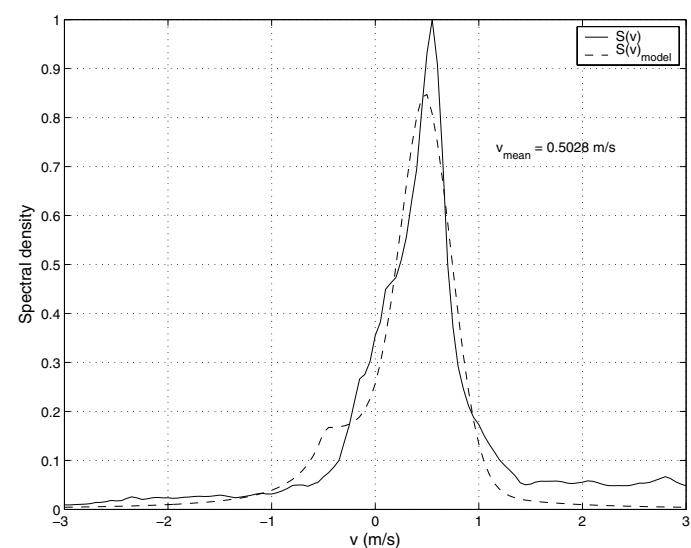

Figure 5-8 $\mathrm{S}(\mathrm{v})$ and $\mathrm{S}(\mathrm{v})_{\text {model }}$ with $\mathrm{k}_{\min }=0.0625 \mathrm{~m}^{-1}$ 


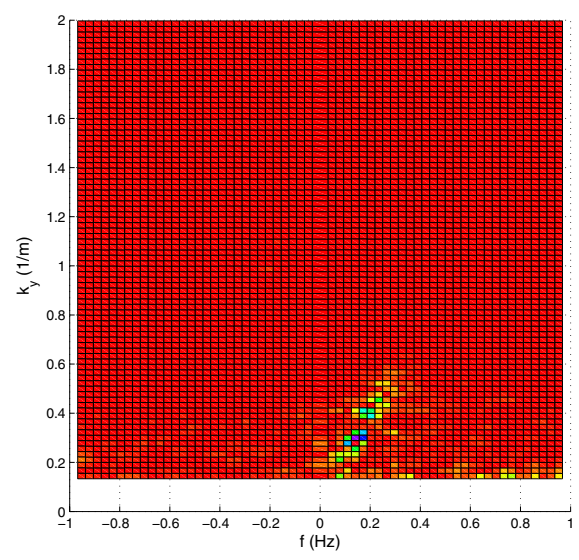

Figure 5-9 $\mathrm{S}(\mathrm{f}, \mathrm{k})$ with $\mathrm{k}_{\min }=0.125 \mathrm{~m}^{-1}$

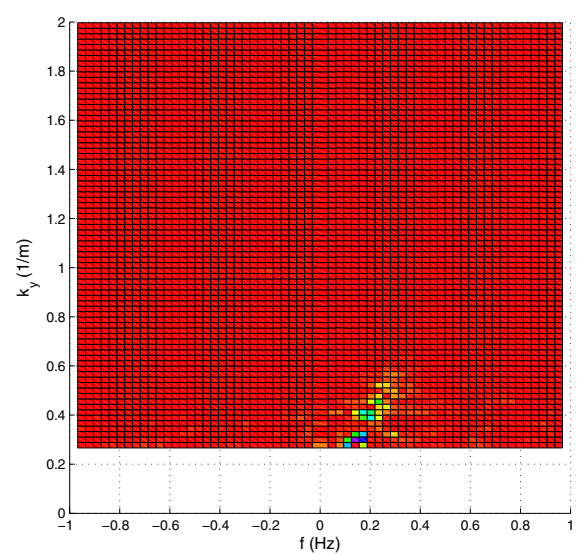

Figure 5-11 $\mathrm{S}(\mathrm{f}, \mathrm{k})$ with $\mathrm{k}_{\min }=0.25 \mathrm{~m}^{-1}$

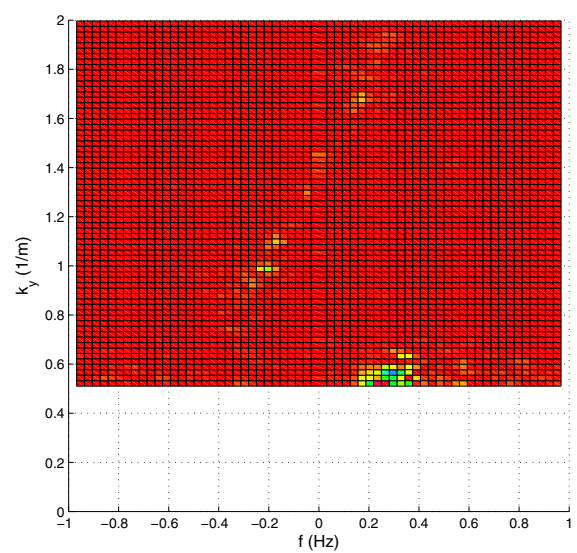

Figure 5-13 $\mathrm{S}(\mathrm{f}, \mathrm{k})$ with $\mathrm{k}_{\min }=0.5 \mathrm{~m}^{-1}$

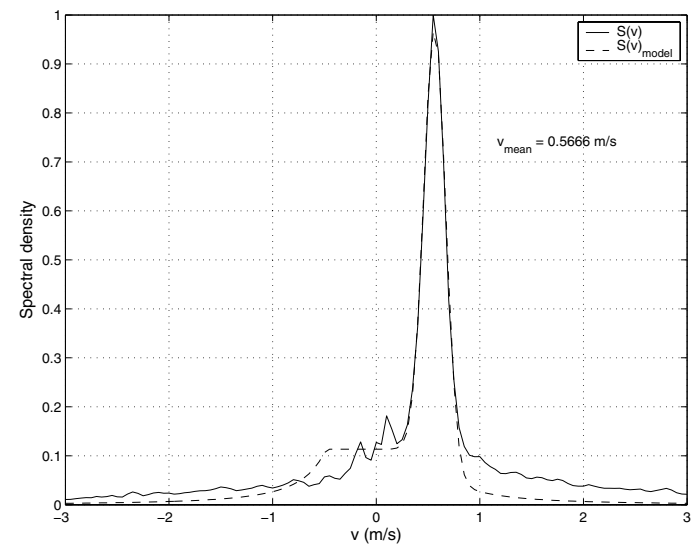

Figure 5-10 $S(v)$ and $S(v)_{\text {model }}$ with $\mathrm{k}_{\min }=0.125 \mathrm{~m}^{-1}$

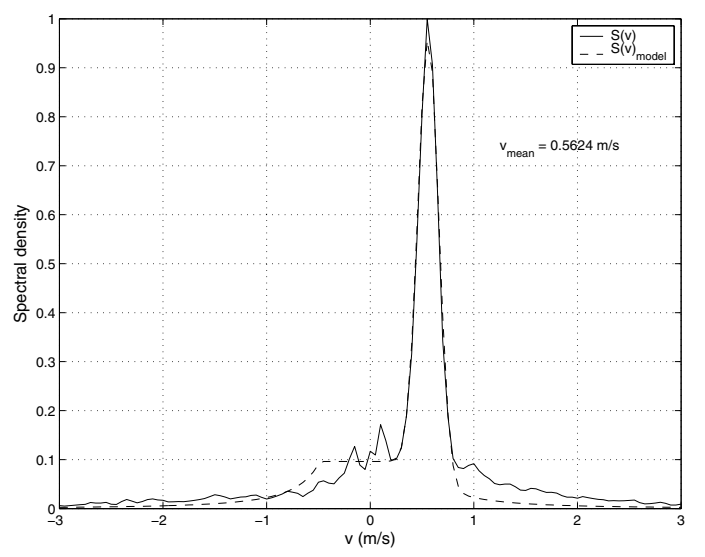

Figure 5-12 $\mathrm{S}(\mathrm{v})$ and $\mathrm{S}(\mathrm{v})_{\text {model }}$ with $\mathrm{k}_{\min }=0.25 \mathrm{~m}^{-1}$

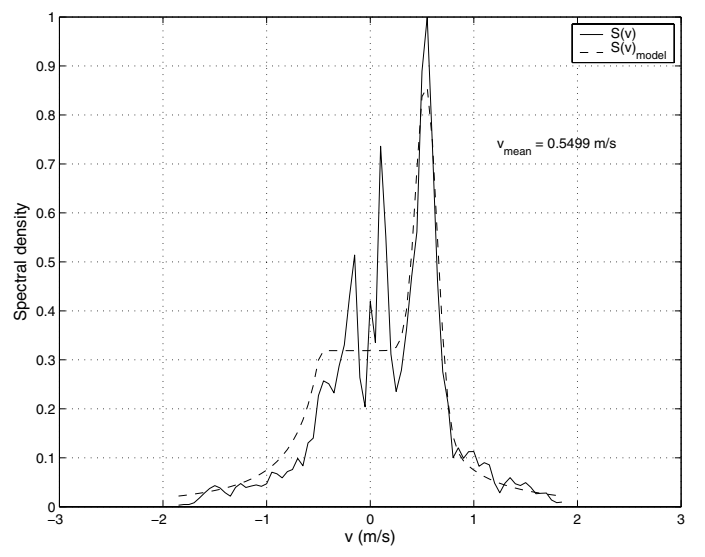

Figure 5-14 $\mathrm{S}(\mathrm{v})$ and $\mathrm{S}(\mathrm{v})_{\text {model }}$ with $\mathrm{k}_{\min }=0.5 \mathrm{~m}^{-1}$ 


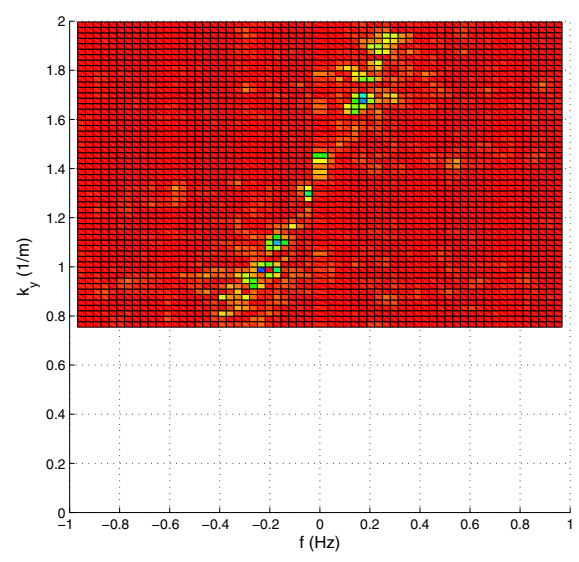

Figure 5-15 $\mathrm{S}(\mathrm{f}, \mathrm{k})$ with $\mathrm{k}_{\min }=0.75 \mathrm{~m}^{-1}$

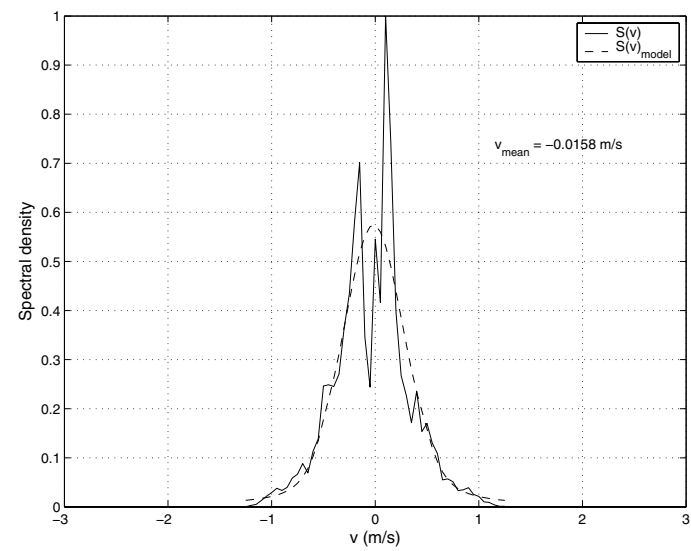

Figure $5-16 \mathrm{~S}(\mathrm{v})$ and $\mathrm{S}(\mathrm{v})_{\text {model }}$ with $\mathrm{k}_{\min }=0.75 \mathrm{~m}^{-1}$

We have to be careful when cutting off low wavenumbers, because by doing this, we increase the influence of the background noise. A safe value of the minimum wavenumber has to be chosen, that cuts off the high spectral density from the passing breaking waves and still neglects the influence of the background noise. The smallest possible minimum wavenumber thus has to be chosen, above a minimum cut-off level for breaking waves, so that the influence of the longshore moving foam patterns is the highest in the spectrum.

The results of the different runs are given in Table 5-4 and Figure 5-17 to Figure 5-20.

\begin{tabular}{|l|l|l|l|l|}
\hline Value $\mathrm{k}_{\min }\left(\mathrm{m}^{-1}\right)$ & $\Delta \mathrm{V}_{\mathrm{rms}}(\mathrm{m} / \mathrm{s})$ & $\Delta \mathrm{V}_{\text {mean }}(\mathrm{m} / \mathrm{s})$ & $\sigma_{\Delta \mathrm{V}}(\mathrm{m} / \mathrm{s})$ & Corr. Coeff. (-) \\
\hline 0.0625 & 0.60 & 0.37 & 0.48 & 0.29 \\
\hline 0.125 & 0.44 & 0.26 & 0.36 & 0.35 \\
\hline 0.25 (default) & 0.25 & 0.18 & 0.18 & 0.52 \\
\hline 0.5 & 0.20 & 0.15 & 0.14 & 0.52 \\
\hline
\end{tabular}

Table 5-4 Quality values for different values of the minimum wavenumber

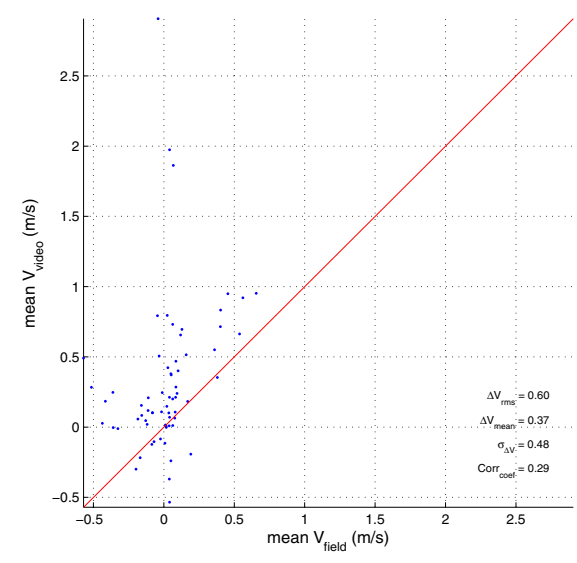

Figure 5-17 Mean $\mathrm{V}_{\text {video }}$ plotted against mean $\mathrm{V}_{\mathrm{gt}}$ with $\mathrm{k}_{\min }=0.0625 \mathrm{~m}^{-1}$

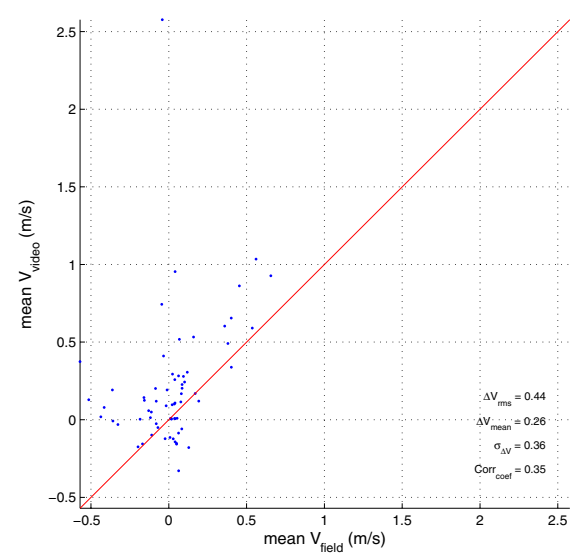

Figure 5-18 Mean $\mathrm{V}_{\text {video }}$ plotted against mean $\mathrm{V}_{\mathrm{gt}}$ with $\mathrm{k}_{\min }=0.1250 \mathrm{~m}^{-1}$ 


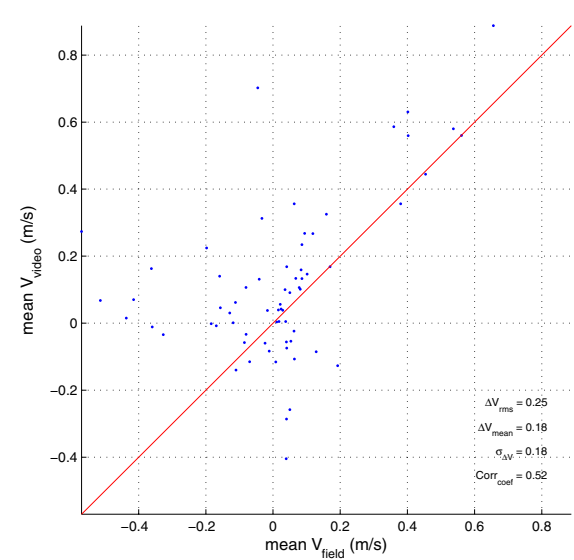

Figure 5-19 Mean $\mathrm{V}_{\text {video }}$ plotted against mean $\mathrm{V}_{\mathrm{gt}}$ with $\mathrm{k}_{\min }=0.2500 \mathrm{~m}^{-1}$

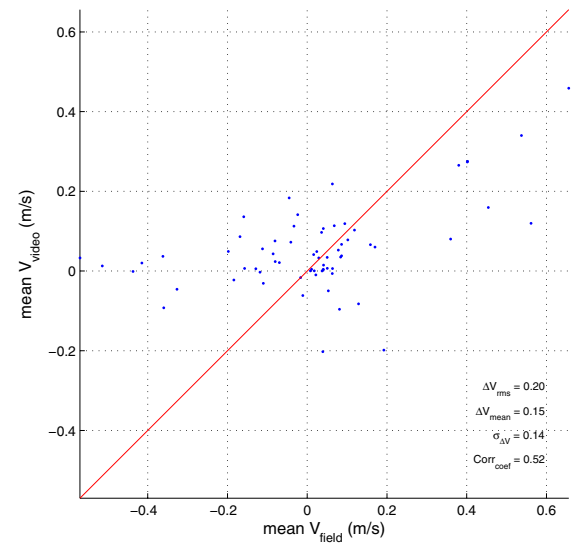

Figure 5-20 Mean $\mathrm{V}_{\text {video }}$ plotted against mean $\mathrm{V}_{\mathrm{gt}}$ with $\mathrm{k}_{\min }=0.5000 \mathrm{~m}^{-1}$

As a higher value of the minimum wavenumber threshold is chosen, the error values decrease considerably. The correlation coefficients increase for higher values of $\mathrm{k}_{\min }$. Between $\mathrm{k}_{\min }=0.25 \mathrm{~m}^{-1}$ and $\mathrm{k}_{\min }=0.50 \mathrm{~m}^{-1}$, there is no difference in correlation coefficient. A $\mathrm{k}_{\min }$ of $0.50 \mathrm{~m}^{-1}$ seems to be giving the best results, but when interpreting the meaning of the minimum wavenumber, it can be seen that a very large minimum wavenumber threshold will result in the recognition of the wrong pattern, i.e. the background noise instead of the longshore moving foam patches. A minimum wavenumber value of $0.25 \mathrm{~m}^{-1}$ is advised for further use of the OCM along the Holland coast, because this value gives the best combination of error values and correlation coefficient, together with the preservation of useful data.

\subsubsection{Probability of the model fit}

In section 3.2, a model spectrum is fitted to the velocity spectrum, to obtain an estimate of the mean velocity. The quality of the model fit can be expressed in $\chi^{2}$, which is the sum of the squares of the normalized differences between the data spectrum and the model spectrum. From this, the probability P of the model fit can be determined.

Only two values different from the default value of the probability are tested as threshold values. This parameter is not expected to play a major role in the reliability of the OCM, but it is stressed to analyse a threshold not equal to zero, with which the data with the worst model fit will be filtered out. Data points below the threshold are excluded from the analysis. Looking at Figure 5-21, it can be seen that most data points have a probability of the model fit of zero or one. The data points located in between have a relatively small $\mathrm{V}_{\text {diff. }}$. From Figure 5-22, we can see that approximately 55\% of the data points has a probability of the model fit of zero. By plotting the mean probability per time series against time we can see if a relation exists between the probability of the model fit and the wave conditions at that time (see Figure 2-17 to Figure 2-20). It can be seen that the spreading of the probability values on April $9^{\text {th }}$ and $10^{\text {th }}$ is quite large, compared to the spreading on April $14^{\text {th }}$, where the mean probability is quite low. Relatively large wave heights are observed on April $10^{\text {th }}$, together with fairly large wave periods. On April $9^{\text {th }}$ and $14^{\text {th }}$, wave heights and wave periods were small. The effect of the wave conditions on the probability of the model fit is not convincing from these data. 


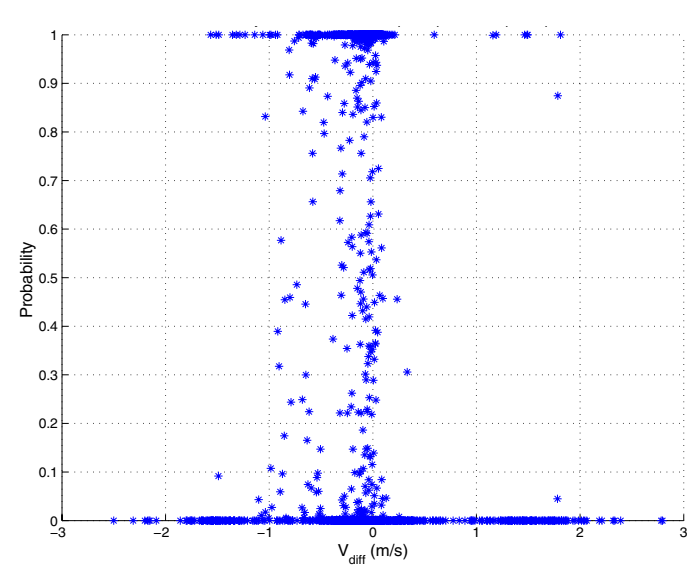

Figure 5-21 Probability of the model fit for each individual velocity estimate $\mathrm{v}_{\mathrm{i}}$ plotted against $\mathrm{V}_{\text {diff }}$

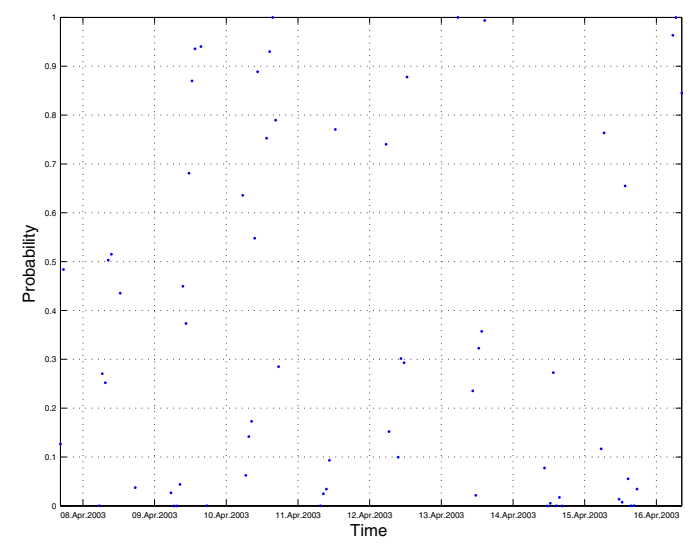

Figure 5-23 Stack averaged probablity plotted against time

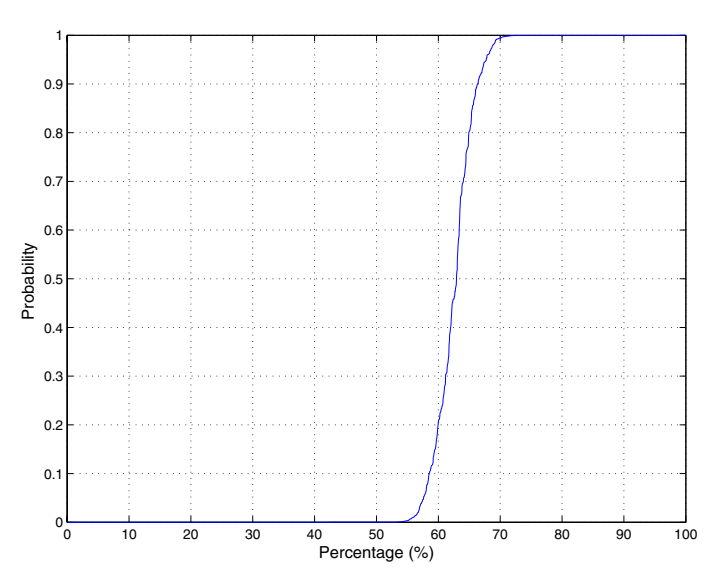

Figure 5-22 Percentage of data points with a certain probability

Threshold values of 0.1 and 0.9 are tested. A testvalue of 0.1 is chosen to cut off data with a large $\left|\mathrm{V}_{\text {diff }}\right|$ and a bad quality of the model fit, as can be seen from Figure 5-21. A value of 0.9 will be tested to only include data in the analysis with a large probability of the model fit to the observed spectrum and because a threshold of 0.9 has been shown to give good results with the OCM in the analysis of the Duck data set (pers. comm. Chris Chickadel).

\begin{tabular}{|l|l|l|l|l|}
\hline $\begin{array}{l}\text { Value } \\
\text { Probability }\end{array}$ & $\Delta \mathrm{V}_{\text {rms }}(\mathrm{m} / \mathrm{s})$ & $\Delta \mathrm{V}_{\text {mean }}(\mathrm{m} / \mathrm{s})$ & $\sigma_{\Delta \mathrm{V}}(\mathrm{m} / \mathrm{s})$ & Corr. Coeff. (-) \\
\hline 0 (default) & 0.25 & 0.18 & 0.18 & 0.52 \\
\hline 0.1 & 0.27 & 0.19 & 0.20 & 0.56 \\
\hline 0.9 & 0.28 & 0.20 & 0.20 & 0.61 \\
\hline
\end{tabular}

Table 5-5 Quality values for different values of a threshold for the probability of the model fit

Table 5-5 shows the error values and the correlation coefficient for different values of a threshold for the probability of the model fit to the observed spectrum. The difference in the error values is small, but a small increase in the errors can be seen when increasing the threshold value, which is counter-intuitive. The correlation coefficient increases also with increasing threshold values, which expresses a better linear relation between the measured and the video data for increasing values of the threshold for the probability of the model fit. 
It can be questioned if the probability of the model fit is an appropriate threshold to improve the accuracy of the velocity estimate by the OCM.

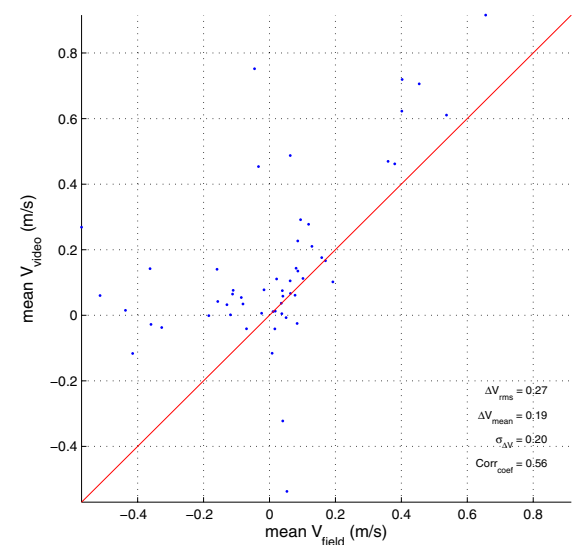

Figure 5-24 Mean $V_{\text {video }}$ plotted against mean $V_{g t}$ with Probability $=0.1$

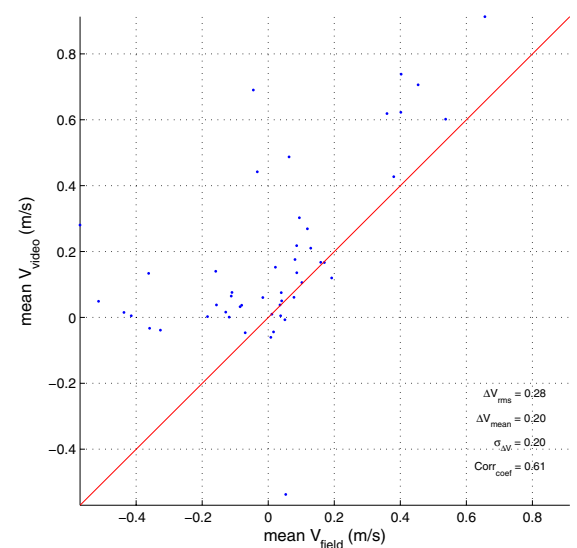

Figure 5-25 Mean $\mathrm{V}_{\text {video }}$ plotted against mean $\mathrm{V}_{\mathrm{gt}}$ with Probability $=0.9$

\subsubsection{Confidence interval of the mean velocity}

In section 3.2, an explanation is given of the fit of a model of the velocity spectrum, $S_{\mathrm{m}}(\mathrm{v})$, to the observed spectrum, $\mathrm{S}(\mathrm{v})$, using a non-linear least-squares routine. The model depends on the four parameters $\mathrm{A}_{\text {foam }}, \bar{v}, \sigma_{\text {foam }}$ and $\mathrm{A}_{\text {noise. }}$ The fitting routine uses the GaussNewton method to iterate to a best-fit solution in the least squares sense. A merit function, $\mathrm{M}(\beta)$, is established which measures the sum of the squared differences between the model and the data, where $\beta$ represents the parameter space for the model. A best-fit solution, $\hat{\beta}$, contains the best-fit settings of the four model parameters. At the same time, a 95\% confidence interval for each parameter is calculated. This confidence interval, $\mathrm{CI}_{\text {span }}$, expresses the values the $\hat{\beta}$ parameters can take while still providing a 95\% reliable fit of the model to the observed spectrum. When the observed spectrum is of very poor quality, the best-fit solution of the model fit to the observed spectrum will be poor as well. An entirely different set of $\hat{\beta}$ parameters will provide a fit that is almost as poor as the best-fit solution and that will be almost as reliable as well. The $95 \%$ confidence interval of the $\hat{\beta}$ parameters of a poor best-fit solution thus has a large width. A best-fit solution of excellent quality will have a very small $95 \%$ confidence interval of the $\hat{\beta}$ parameters. A threshold value of the $95 \%$ confidence interval of one of these parameters thus will tell us something about the quality of the observed spectrum. In this paragraph a threshold value of the $95 \%$ confidence interval of the $\hat{\beta}$ parameter of direct interest, $\mathrm{V}_{\text {mean }}$, is examined. 


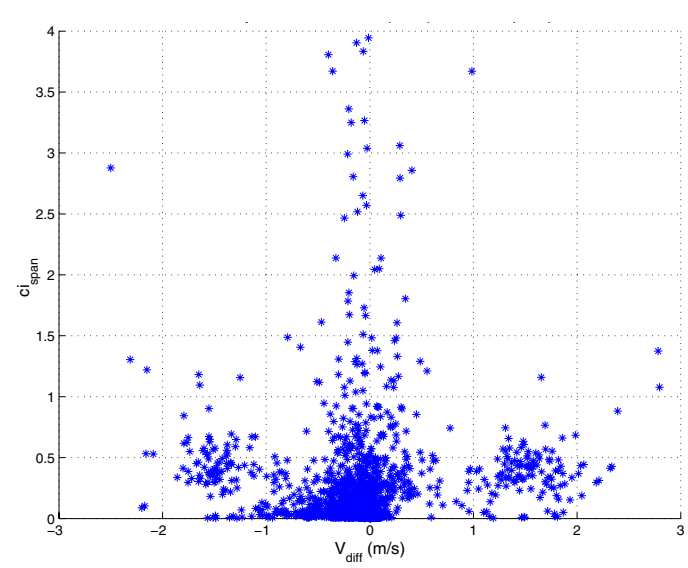

Figure 5-26 $\mathrm{CI}_{\text {span }}$ for each time step of each OCM time series plotted against $\mathrm{V}_{\text {diff }}$

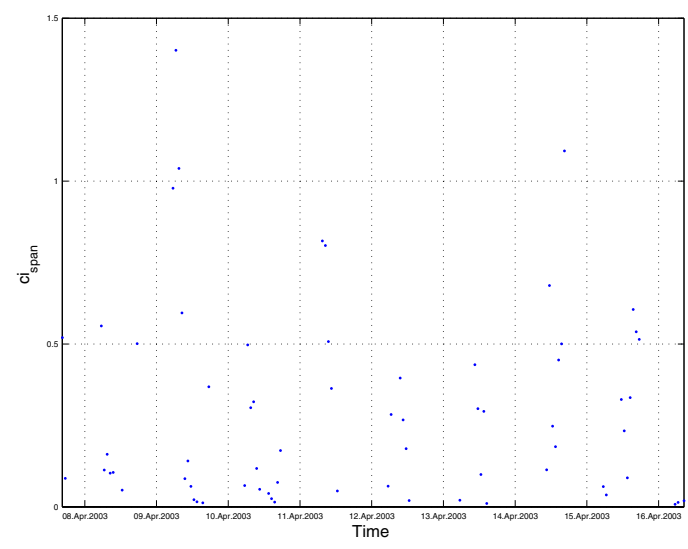

Figure 5-27 Mean $\mathrm{CI}_{\text {span }}$ per time series plotted against time

In Figure 5-26, the $\mathrm{CI}_{\text {span }}$ values are shown for each time step of each time series estimated by the OCM. The majority of data points has a $\mathrm{CI}_{\text {span }}$ lower than $0.75 \mathrm{~m} / \mathrm{s}$. Three groups of estimates can be distinguished in this area, two groups with large values of $\left|V_{\text {diff }}\right|$, and one very large group with small values of $\left|\mathrm{V}_{\text {diff }}\right|$. When a threshold is employed, data with a value higher than the threshold value are excluded. When the threshold is chosen at a very low value of the $95 \%$ confidence interval, for example below $0.1 \mathrm{~m} / \mathrm{s}$, much good data (data with a small $\left|\mathrm{V}_{\text {diff }}\right|$ ) will be lost. Small values are tested, to see how exclusion of these data affects the error values and the correlation coefficient. Judging from Figure 5-26, it is expected that a $\mathrm{CI}_{\text {span }}$ of $0.5 \mathrm{~m} / \mathrm{s}$ will result in a bad quality of the velocity estimation by the OCM, because the two groups of data points with large values of $\left|\mathrm{V}_{\text {diff }}\right|$ are included, which will cause the large error values and the small correlation coefficient.

Four different values of $\mathrm{CI}_{\text {span }}$ are tested. Values below the threshold value are accepted. The results of the tests are shown in Table 5-6 and in Figure 5-28 to Figure 5-31.

\begin{tabular}{|l|l|l|l|l|}
\hline Value $\mathrm{CI}_{\text {span }}(\mathrm{m} / \mathrm{s})$ & $\Delta \mathrm{V}_{\text {rms }}(\mathrm{m} / \mathrm{s})$ & $\Delta \mathrm{V}_{\text {mean }}(\mathrm{m} / \mathrm{s})$ & $\sigma_{\Delta \mathrm{V}}(\mathrm{m} / \mathrm{s})$ & Corr. Coeff. (-) \\
\hline 4 (default) & 0.25 & 0.18 & 0.18 & 0.52 \\
\hline 0.5 & 0.27 & 0.19 & 0.20 & 0.44 \\
\hline 0.2 & 0.25 & 0.17 & 0.18 & 0.58 \\
\hline 0.1 & 0.27 & 0.18 & 0.19 & 0.62 \\
\hline 0.05 & 0.26 & 0.18 & 0.19 & 0.62 \\
\hline
\end{tabular}

Table 5-6 Quality values for different values of the maximum confidence interval of the mean velocity of the model fit

The error values of the different $\mathrm{CI}_{\text {span }}$ threshold values do not change much when different values of the parameter below $0.2 \mathrm{~m} / \mathrm{s}$ are chosen. The only value that shows a noticeable variation is the correlation coefficient. As the $\mathrm{CI}_{\text {span }}$ threshold value is chosen smaller, the correlation coefficient increases. It can thus be seen that a tighter confidence interval band of the accepted data results in a more nearly linear relation between the measured and the video data. $\mathrm{A} \mathrm{CI}_{\mathrm{span}}$ value of $0.5 \mathrm{~m} / \mathrm{s}$ gives higher error values and a much lower correlation than both the default setting and the other tested $\mathrm{CI}_{\text {span }}$ values, which was already expected. 


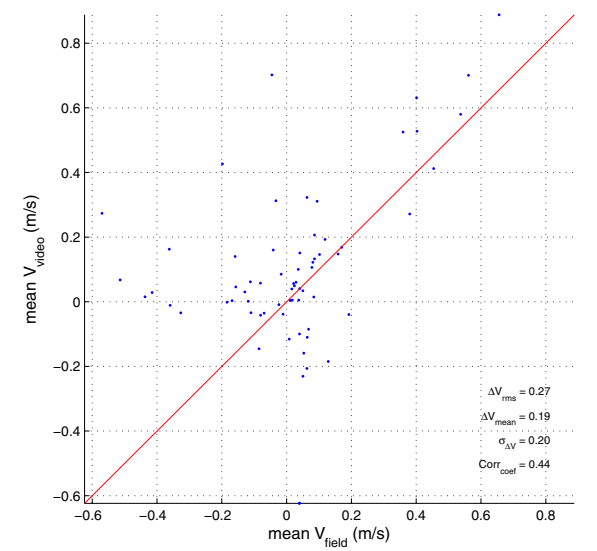

Figure 5-28 Mean $\mathrm{V}_{\text {video }}$ plotted against mean $\mathrm{V}_{\mathrm{gt}}$ with C.I.span $=0.5 \mathrm{~m} / \mathrm{s}$

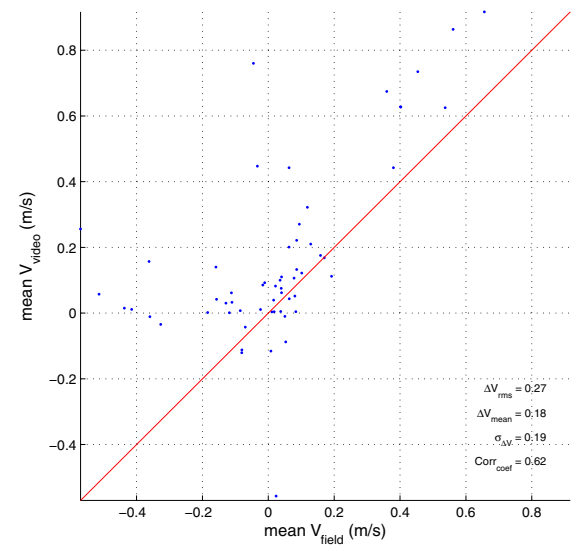

Figure 5-30 Mean $\mathrm{V}_{\text {video }}$ plotted against mean $\mathrm{V}_{\mathrm{gt}}$ with C.I.span $=0.1 \mathrm{~m} / \mathrm{s}$

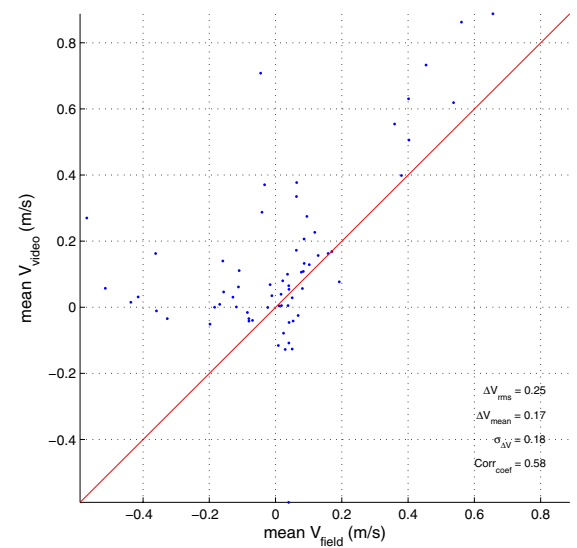

Figure 5-29 Mean $\mathrm{V}_{\text {video }}$ plotted against mean $\mathrm{V}_{\mathrm{gt}}$ with C.I.span $=0.2 \mathrm{~m} / \mathrm{s}$

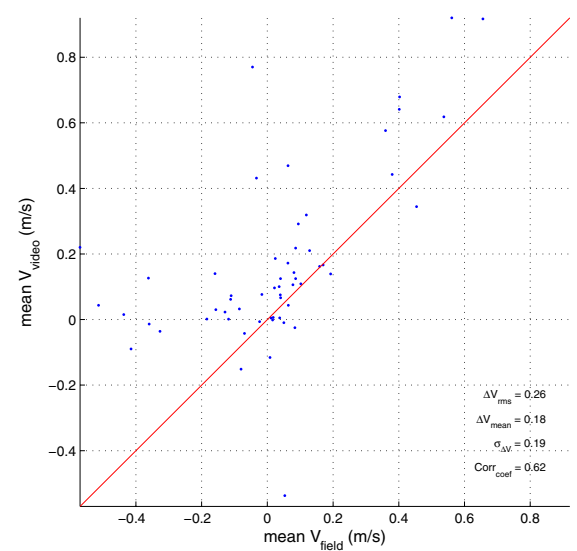

Figure 5-31 Mean $\mathrm{V}_{\text {video }}$ plotted against mean $\mathrm{V}_{\mathrm{gt}}$ with C.I.span $=0.05 \mathrm{~m} / \mathrm{s}$

\subsubsection{Intensity range}

When a histogram of the intensities in a time stack would be made, the range between the $95 \%$ and the $50 \%$ intensity value can be called $I_{\text {range. }}$ This parameter is a measure for the contrast and the amount of breaking in the time stack. When defining a threshold value for this $\mathrm{I}_{\text {range }}$, a selection of useful data can be made by the model, based on the contrast and the amount of breaking in the time stack. Previous research (Chickadel, 2002) showed that the best results were obtained with a value of $I_{\text {range }}=40$. This value resulted from tests with the Duck data set. Here, the influence of a threshold of $I_{\text {range }}$ is examined with the Noordwijk data set to find a useful value for this location. 


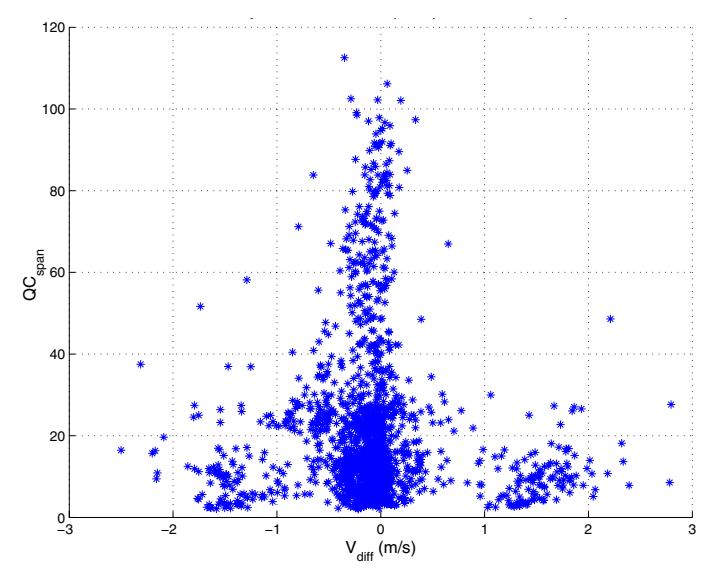

Figure 5-32 $\mathrm{I}_{\text {range }}$ for each time step of each OCM time series plotted against $\mathrm{V}_{\text {diff }}$

Figure 5-32 shows the $\mathrm{I}_{\text {range }}$ for each time step of each time series estimated by the OCM. It can be seen that for small values of $\mathrm{I}_{\text {range }}$, i.e. when there is only little or no breaking and contrast, the range of values of $V_{\text {diff }}$ is large. As the $I_{\text {range }}$ increases, values of $V_{\text {diff }}$ are consequently closer to zero, thus the velocity estimates by the OCM are more stable for larger values of $\mathrm{I}_{\text {range }}$. We expect smaller error values and a larger correlation coefficient for larger values of $\mathrm{I}_{\text {range. }}$. When a high threshold of the $\mathrm{I}_{\text {range }}$ is employed, also a large amount of data points with a small value of $\mathrm{V}_{\text {diff }}$ are excluded, besides the bad data with large $\mathrm{V}_{\text {diff. }}$. This will have a negative effect on the error values and the correlation coefficient for larger values of $I_{\text {range. }}$.

Five different threshold values will be tested to investigate the influence of the threshold value of $I_{\text {range }}$ on the quality of the velocity estimation by the OCM. The results of the tests are shown in Table 5-7 and Figure 5-33 to Figure 5-37.

\begin{tabular}{|l|l|l|l|l|}
\hline $\begin{array}{l}\text { Value Intensity } \\
\text { range }\end{array}$ & $\Delta \mathrm{V}_{\text {rms }}(\mathrm{m} / \mathrm{s})$ & $\Delta \mathrm{V}_{\text {mean }}(\mathrm{m} / \mathrm{s})$ & $\sigma_{\Delta \mathrm{V}}(\mathrm{m} / \mathrm{s})$ & Corr. Coeff. (-) \\
\hline 0 (default) & 0.25 & 0.18 & 0.18 & 0.52 \\
\hline 10 & 0.27 & 0.18 & 0.20 & 0.55 \\
\hline 20 & 0.29 & 0.19 & 0.22 & 0.63 \\
\hline 30 & 0.30 & 0.20 & 0.23 & 0.57 \\
\hline 40 & 0.28 & 0.18 & 0.22 & 0.56 \\
\hline 50 & 0.34 & 0.23 & 0.25 & 0.62 \\
\hline
\end{tabular}

Table 5-7 Quality values for different values of the minimum intensity range

As opposed to what was expected from Figure 5-32, the error values increase for increasing values of the $I_{\text {range }}$ threshold. This is caused by the fact that besides bad quality data, also a large amount of data points with a very small $\mathrm{V}_{\text {diff }}$ are excluded from the analysis. An $\mathrm{I}_{\text {range }}$ threshold of 50 yields the largest error values, with a root-mean-square error of $0.34 \mathrm{~m} / \mathrm{s}$, which is large compared to mean observed longshore current velocities in the range of 0.3 to $0.5 \mathrm{~m} / \mathrm{s}$. The correlation coefficient has a non linear course, with no obvious increase or decrease with increasing threshold values of $I_{\text {range. }}$ The largest correlation coefficient $(0.63)$ is found for an $I_{\text {range }}$ threshold of 20. Figure 5-33 to Figure 5-37 show that for increasing 
values of the $\mathrm{I}_{\text {range }}$ threshold, the set of velocity estimates by the OCM decreases considerably. A value 10 still provides a considerable set of velocity estimates, which have quite a wide spreading across the plot. With a value of 30 , the set is almost halved, but the velocity estimates appear to line up more closely to the line of equality. Again, it can be questioned if the intensity range is the appropriate threshold to improve the accuracy of the velocity estimate by the OCM.

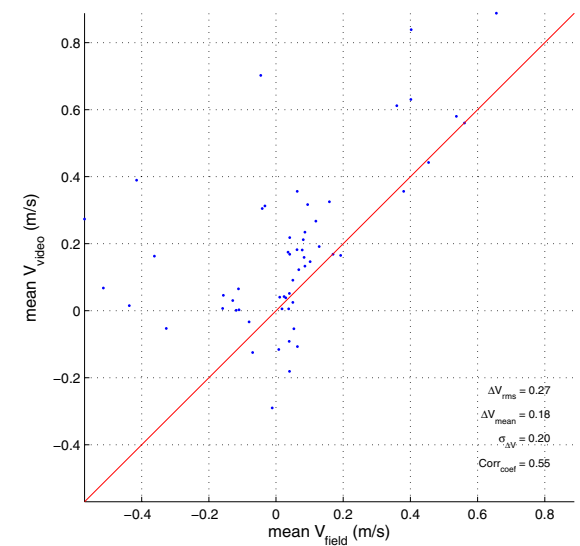

Figure 5-33 Mean $\mathrm{V}_{\text {video }}$ plotted against mean $\mathrm{V}_{\mathrm{gt}}$ with $\mathrm{I}_{\text {range }}=10$

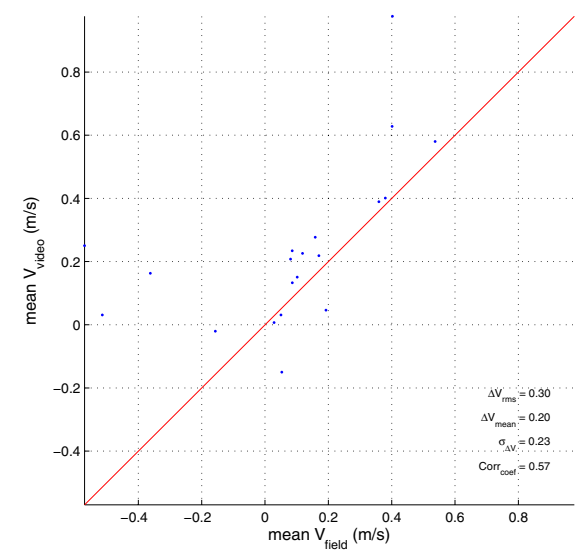

Figure 5-35 Mean $\mathrm{V}_{\text {video }}$ plotted against mean $\mathrm{V}_{\mathrm{gt}}$ with $\mathrm{I}_{\text {range }}=30$

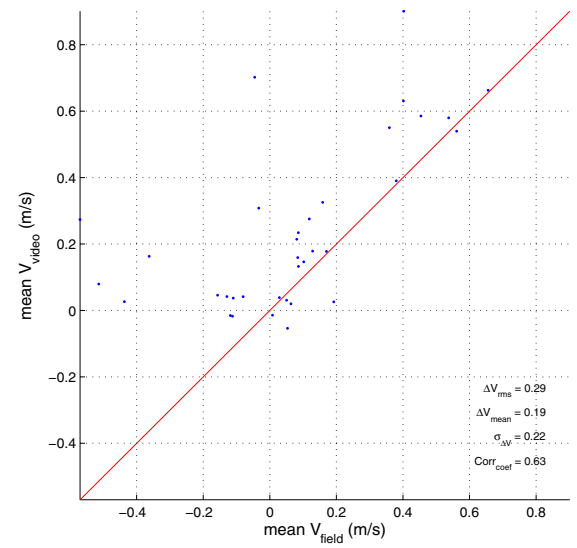

Figure 5-34 Mean $\mathrm{V}_{\text {video }}$ plotted against mean $\mathrm{V}_{\mathrm{gt}}$ with $\mathrm{I}_{\text {range }}=20$

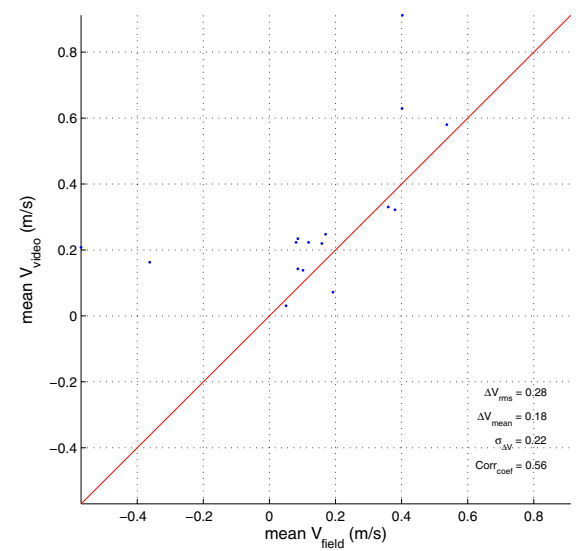

Figure 5-36 Mean $\mathrm{V}_{\text {video }}$ plotted against mean $\mathrm{V}_{\mathrm{gt}}$ with $\mathrm{I}_{\text {range }}=40$ 


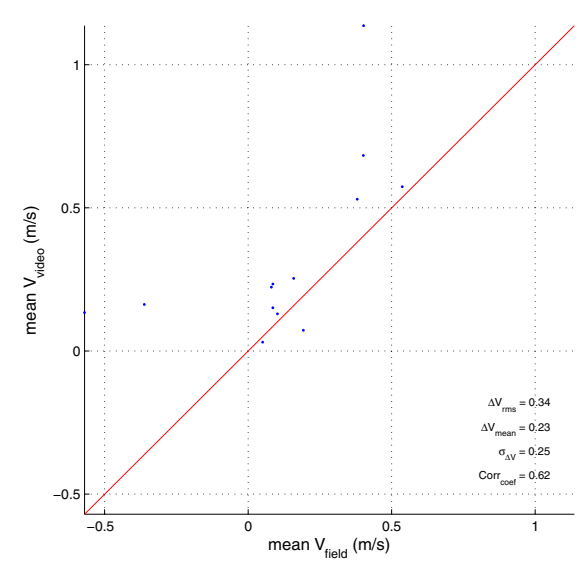

Figure 5-37 Mean $\mathrm{V}_{\text {video }}$ plotted against mean $\mathrm{V}_{\mathrm{gt}}$ with $\mathrm{I}_{\text {range }}=50$

\subsection{Conclusions: best settings}

Based on the calibration and validation carried out in the previous paragraphs, values of the model parameters are chosen, with which a final run will be made, to evaluate the influence of the calibration procedure on the quality of the velocity estimates by the OCM. The calibration and validation have resulted in the values shown in Table 5-8.

\begin{tabular}{|l|l|l|}
\hline Model parameter & Default value & Final run value \\
\hline Time window & $32 \mathrm{~s}$ & $16 \mathrm{~s}$ \\
\hline Time shift & $16 \mathrm{~s}$ & $8 \mathrm{~s}$ \\
\hline Minimum wavenumber & $0.25 \mathrm{~m}^{-1}$ & $0.25 \mathrm{~m}^{-1}$ \\
\hline Probability of model fit & 0 & 0.1 \\
\hline 95\% confidence interval & $4 \mathrm{~m} / \mathrm{s}$ & $0.1 \mathrm{~m} / \mathrm{s}$ \\
\hline Intensity range & 0 & 20 \\
\hline
\end{tabular}

Table 5-8 Values of the model parameters used in the final run

The final run uses a conservative approach of choosing the model parameter values, which means that the parameters are chosen in a way, that the data that are included have the smallest error values and the largest correlation coefficient as possible, so that an optimal accuracy of the velocity estimate is reached. A $\mathrm{T}_{\mathrm{w}}$ of $16 \mathrm{~s}$ and a $\mathrm{T}_{\mathrm{s}}$ of $8 \mathrm{~s}$ are used because these values give the best results while being the most economical, even though the difference with other values is very small. The minimum wavenumber value is chosen to retain its default value, because this value gives the best combination of error values and correlation coefficient, together with the maintenance of useful data. With a minimum wavenumber of $0.50 \mathrm{~m}^{-1}$, the error values are better, but this value does not recognise the longshore moving foam patches, but only the background noise, which has a smaller velocity than the longshore moving foam patches. The threshold for the probability of the model fit is chosen at a value of 0.1 , which includes a large amount of data points with a good quality of the velocity estimate, while the quality of the model fit to the observed spectrum is poor. A $95 \%$ confidence interval of the mean velocity is chosen to be $0.1 \mathrm{~m} / \mathrm{s}$, which has shown to give the best combination of error values and correlation coefficient. An $\mathrm{I}_{\text {range }}$ of 20 has also shown to yield the best results. 


\begin{tabular}{|l|l|l|l|l|}
\hline Value model parameters & $\Delta \mathrm{V}_{\text {rms }}(\mathrm{m} / \mathrm{s})$ & $\Delta \mathrm{V}_{\text {mean }}(\mathrm{m} / \mathrm{s})$ & $\sigma_{\Delta \mathrm{V}}(\mathrm{m} / \mathrm{s})$ & Corr. Coeff. (-) \\
\hline Reference & 0.25 & 0.18 & 0.18 & 0.52 \\
\hline Final run & 0.31 & 0.21 & 0.22 & 0.62 \\
\hline
\end{tabular}

Table 5-9 Quality values for the default and the final run

As we can see from Table 5-9, the correlation coefficient in the final run has increased considerably, compared to the reference run. This means that the linear correlation between the velocity estimate from the OCM and the ground truth velocity has improved. The error values show some increase too, but the difference is much smaller.

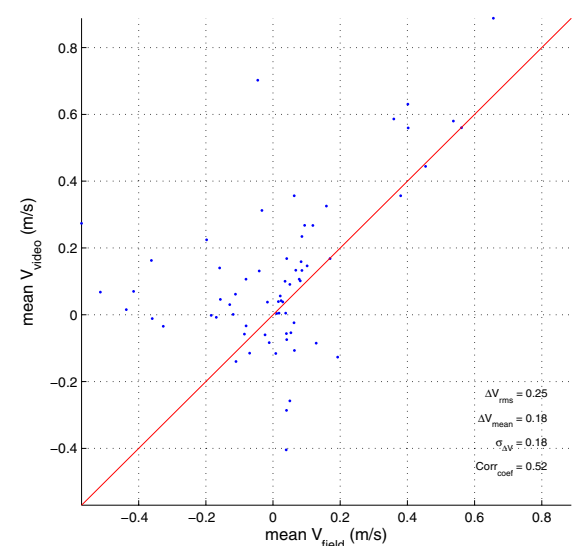

Figure 5-38 Mean $\mathrm{V}_{\text {video }}$ plotted against mean $\mathrm{V}_{\mathrm{gt}}$ for the reference run

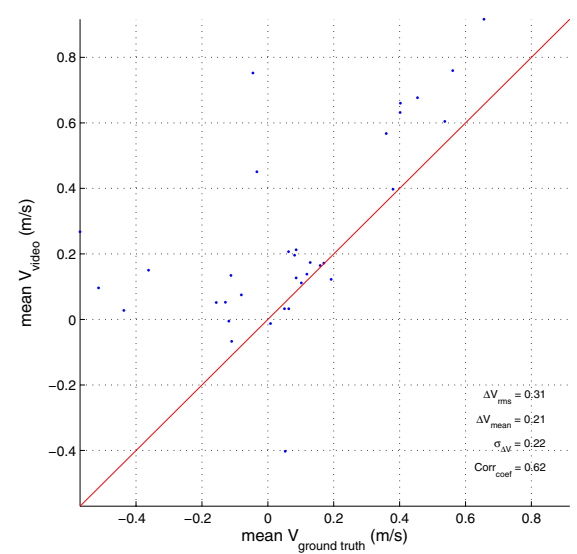

Figure 5-39 Mean $\mathrm{V}_{\text {video }}$ plotted against mean $\mathrm{V}_{\mathrm{gt}}$ for the final run

Figure 5-38 and Figure 5-39 show the resulting velocity estimates from the OCM together with the ground truth velocities for the reference and the final run. It is evident that a smaller number of data points is left from the final run. Most of the outliers are still present, but the main part of the remaining data points lie along the 1:1 line, with a small offset. For positive (i.e. southward-directed) velocities, video-derived velocities consistently overestimate the corresponding ground truth measurements. This can possibly be explained from the observation that the OCM estimates surface velocities, whereas the EMF measures a velocity in the water column, at about $25 \mathrm{~cm}$ above the sea bed. A mean longshore current, flowing over the bottom, will develop a shear profile described by a logarithmic boundary layer. The comparison data set, containing a surface measurement and one in the interior, cannot be used to test for a log-layer. However, vertical shear in the upper portion of the water column due to bottom boundary layer effects has been observed to be small, on the order of a few $\mathrm{cm} / \mathrm{s}$ (Chickadel et al., 2003). The results from the final run show a rootmean-square value of $0.31 \mathrm{~m} / \mathrm{s}$, which cannot solely be caused by the influence of the bottom. The alongshore component of the wind stress can cause a surface boundary layer. It is reasonable to expect $\Delta \mathrm{V}$ to be in the same direction as, and have a magnitude that varies with, the alongshore component of the wind stress (Chickadel et al., 2003).

The accuracy of the velocity estimates from the OCM is still rather weak, but a larger correlation between the velocity estimates from the OCM and the ground truth velocities is reached. The error values may be decreased by correcting for the difference between interior current and surface current velocities (see section 7.2). Another reason for the weak 
accuracy can be that the occurring longshore velocities are small compared to the background noise. The noise/signal ratio is thus unfavourable.

We have seen that some of the model parameters tested in this chapter did not yield the expected results. It can be questioned if these parameters are appropriate for improving the accuracy. Research should be done to find model parameters, other than the parameters tested in this research, to improve the accuracy of the OCM. For instance, a parameter can be developed, that gives information about the 'grassiness' of the S(v) spectrum. We have seen that when more than one peak occurs, the velocity estimate of the spectrum becomes less accurate, due to the wrong signal that is picked up. The 'grassiness' can be expressed as $\mathrm{G}=\mathrm{S}_{\text {peak }} / \mathrm{S}_{\text {peak-1 }}$, which means the normalised spectral density of the highest peak divided by the normalised spectral density of the second highest peak. When $G$ is large, the spectrum has one well pronounced peak and the estimate is accurate. When $G$ is small, the spectrum is 'grassy' and the estimate is of poor accuracy. Another option is to apply a parameter that looks at the consistency of the individual velocity estimates $v_{i}$. When for instance $90 \%$ of the individual velocity estimates does not pass the quality criteria with respect to P, C.I. and

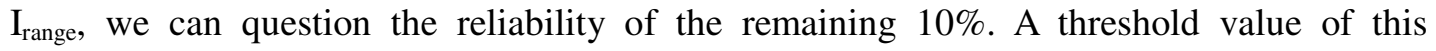
percentage might improve the accuracy of the resulting stack averaged velocity estimates by the OCM. Furthermore, the standard deviation of $v_{i}$ might give information on the reliability of the individual velocity estimates. More research is needed to investigate other possibilities of quality parameters to improve the accuracy of the OCM. 


\section{Discussion on the utility of the OCM}

\section{I Introduction}

Now that we have investigated the applicability and the accuracy of the OCM, it is important to take a further look at the utility of the OCM, which means the way the OCM can be applied by the coastal manager or by scientists. The present study has been carried out in the framework of the CoastView project. The longshore current velocities play a role as Coastal State Indicator for swimmer safety hence tourism. Besides that, longshore current velocities play a major role in longshore sediment transport processes and it is of great importance to know the value of these velocities to calculate the longshore sediment transport in order to be able to predict morphological changes. The question is, can we use the OCM for these purposes at the Holland coast and are there any other possible applications of the OCM?

\subsection{Discussion}

\subsection{Utility regarding accuracy}

The present accuracy of the OCM is not good enough to use it as an actual measuring device to measure current velocities for quantitative use, for instance to indicate if it is safe to swim in a certain part of the sea by setting a threshold for the longshore current velocity and using the OCM estimate as an absolute measuring device. It is however possible to estimate the longshore current velocity every 15 minutes with the OCM and to sound the alarm when the velocities start to grow with a certain speed. This way, we do not use the absolute OCM estimates, but their relative values in time. It should be mentioned that the major cause of drowning along the coast has to be found in the cross-shore rip currents rather than in strong longshore currents. The worst thing longshore currents can do is move people, and probably mostly children, along the coast without them noticing. This way children can lose their parents on a crowded beach. The OCM is not able to estimate cross-shore velocities, because no cross-shore signal, indicating the cross-shore velocity, can be recognized from the water surface at locations where rip currents occur.

An alternative utility can be to collect pixel intensity data along a set of longshore arrays. A current velocity can be estimated in each array using the OCM, resulting in a spatial distribution of the longshore current velocity. The absolute values of these velocities may not be very accurate, but the relative values can be. These are important when investigating a spatial distribution. For instance, a cross-shore set of longshore arrays can be designed to obtain the cross-shore distribution of the longshore current velocity (see Figure 6-1 and Figure 6-2). The points are plotted for which an estimate of the longshore current velocity could be made. The arrays that were located at the dry beach or outside the breaker zone do not yield an estimate by the OCM, because no foam patterns are present from which a velocity estimate can be made. The distribution shows a very realistic profile. 


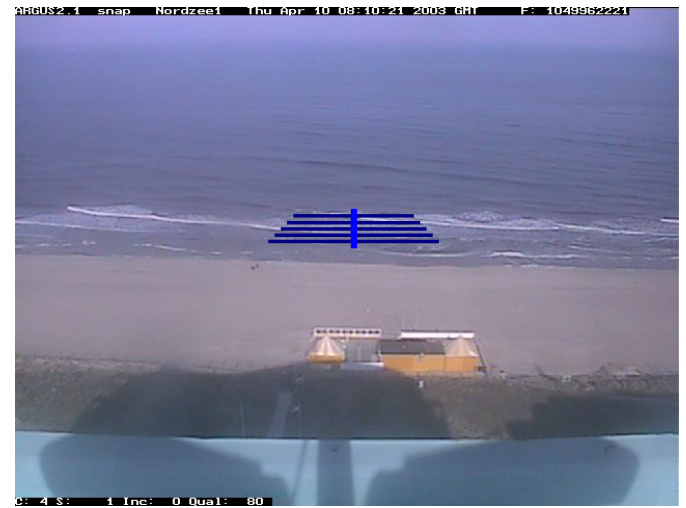

Figure 6-1 Pixel arrays at Noordwijk used for the analysis of the cross-shore distribution of the longshore current. The blue dots show the points in which the estimates are made.

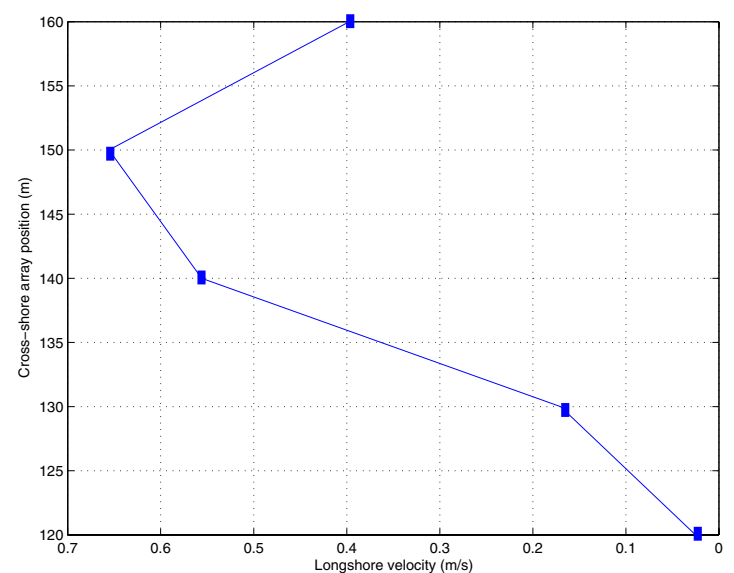

Figure 6-2 Cross-shore distribution of the longshore current velocity

\subsubsection{Spatial utility}

From Chapter 4 we have discovered that the longshore pixel dimension in an image plays a significant role in the applicability of the OCM. This has shown to be a problem with the Egmond data, because the pixel arrays were located too far from the cameras. Only a small part of the range (up to $200 \mathrm{~m}$ at each side) of the Argus station can be used for collection of useful data for the OCM. To improve the spatial utility of the OCM, cameras with a better resolution have to be used to collect the data used in the OCM. Presently, a new generation of Argus cameras is being installed at new Argus stations worldwide, called the Argus III fully-digital system. These cameras will improve the spatial utility of the OCM considerately, meaning that pixel arrays located further from the cameras can be used to collect data for the OCM.

\subsubsection{Temporal utility}

Even if the accuracy of the OCM would be sufficient, the usefulness in using the velocity estimates to alert swimmers for large longshore velocities has to be questioned. The data have to be collected by daylight, transferred to an Argus server and analysed using the OCM. By the time a velocity estimate is made, the day is over and the swimmers have gone home already, hopefully without drowning. However, recently improved telecommunication capabilities like ADSL will allow for real-time access to the data. Also, the OCM can be applied in automated mode, so that a velocity estimate can be made at the site, without having to transfer stack data to a manned computer.

Ideally, a data base has to be set up, containing a history of wind, wave and tidal data and the corresponding velocity estimates along the coast. With forecasts of the wind and the tide, an estimate can be made of the occurring current velocity in de future. The OCM velocity estimates can be used as input data for a longshore current model, such as described in Reiniers, 1999. Together with wave and wind data, a forecast of the longshore current velocity can be made. This forecast can be used to predict longshore current velocities along the beach and to warn swimmers when large longshore current velocities occur. 


\subsubsection{Meteorological utility}

The weather circumstances in The Netherlands are not ideal for everyday use of the OCM. Ideal would be a clear sky, a constant onshore wind, breaking waves producing large and well pronounced foam patches and no rain. In The Netherlands, none of these conditions occur simultaneously. When the sky is clear, usually there is no wind or just a weak wind from the east. When waves are large enough to produce well pronounced foam patches, it often rains, blurring the camera lens. To improve the utility of the OCM, we would like to stimulate the simultaneousness of the conditions mentioned earlier. Because we cannot force the sky, the wind or the rain, the only condition that is left to stimulate is the production of foam patches on the water surface. A progressive idea would be to install a pole in the breaker zone, with a small foam machine attached to it that releases a very white foam patch of for instance $1 \mathrm{~m}$ every $8 \mathrm{~s}$. The pole's location will coincide with an alongshore located pixel array, that will be used to collect data and pick up the foam patterns. This technique may provide a more accurate estimate of the longshore current velocity, to be used for swimming safety, and could also be used at places where no breaking occurs at all, for instance in river and harbour entrances, where flow velocities are of great importance for shipping safety and water depth management. More research has to be done in this field. 


\section{Conclusions and recommendations}

\section{I Conclusions}

\section{Applicability}

Conclusions from Chapter 4:

- The OCM parameter settings developed for Duck are not directly applicable to the two sites at the Holland coast.

- Pixel arrays designed with a pixel spacing that has a value close to the longshore pixel dimension give the best results with the OCM.

- The longshore pixel dimension in an image plays a significant role in the applicability of the OCM. A longshore pixel dimension of $2 \mathrm{~m}$ is too large for the OCM to function in a proper way.

\section{Accuracy}

Conclusions from Chapter 5:

- The accuracy of the velocity estimates from the OCM after calibration and validation is still rather weak, but a larger correlation between the velocity estimates from the OCM and the ground truth velocities has been achieved.

- The OCM estimates show a persistent off-set from the ground truth velocities. This is most probably caused by the difference between surface and interior current due to bottom and wind shear stresses.

- The OCM easily allows for the incorporation of additional quality indicators to judge on the accuracy of a velocity estimate.

\section{Utility}

Conclusions from Chapter 6:

- The accuracy of the OCM is not good enough to use it as an actual measuring device to measure current velocities for quantitative use, for instance to indicate if it is safe to swim in a certain part of the sea, for which an absolute velocity is needed. 
- Only a small part of the range of the Argus station can be used for collection of useful data for the OCM with the current Argus cameras available at the Holland coast because of the pixel dimension problem.

- Using the OCM to obtain a cross-shore distribution of the longshore current velocities yields a realistic cross-shore velocity profile.

- The weather conditions in The Netherlands are not ideal for everyday use of the OCM.

\subsection{Recommendations}

- Further research is needed to get better insight in the spatial range of the OCM and the corresponding longshore pixel dimensions.

- Research is needed to determine the offset factor to correct for the consistent offset of the OCM velocity estimates from the ground truth velocities.

- An inquiry should be made into the average length of foam patches along the Holland coast, to find the optimal value of $\mathrm{k}_{\mathrm{min}}$.

- Research should be done to find quality indicators, other than the parameters tested in this research, to improve the accuracy of the OCM along the Holland coast. For instance, a parameter can be developed, that gives information about the 'grassiness' of the $\mathrm{S}(\mathrm{v})$ spectrum.

- It would be wise to carry out another field experiment, to collect ground truth data with measuring devices that are not vandalized, to make sure that the relation between the OCM velocity estimates and the ground truth velocities is persistent.

- Research has to be done to investigate the possibility of integrating the OCM with a longshore current model.

- Research has to be done to investigate the accuracy of using the OCM in the estimation of a spatial distribution of the longshore current velocity. 


\section{References}

Aarninkhof, S. G. J., M. Caljouw \& M. J. F. Stive (2000). Video-based, quantitative assessment of intertidal beach variability. In: Proc. $27^{\text {th }}$ Int. Conf. on Coastal Engineering, New York: ASCE, pp. 3291-3304.

Aarninkhof, S. G. J. \& R. Holman (1999). Monitoring the Nearshore with Video. Backscatter May 1999, pp. 811.

Aarninkhof, S. G. J. \& K. Kingston (2002). The Argus Runtime Environment, guidelines on Installation and Use. manualArgusTools2002b.doc, Version dd. 27-08-02.

Bendat, J. S. \& A. G. Piersol (1971). Random data: analysis and measurement procedures. John Wiley \& Sons, New York, USA.

Chickadel, C. C. (2002). Measuring Surface Longshore Currents with an Optical Technique. MSc. Thesis Oregon State University.

Chickadel, C. C., Holman, R.A. and Freilich, M. (2003). An optical technique for the measurement of longshore currents. Submitted to Journal of Geophysical Research.

Feddersen, F.. Longshore Currents (1997). Scrips Institute of Oceanography. Lecture notes. wwwccs.ucsd.edu/ falk/lecture5.pdf.

Huntley, D. A., R. T. Guza \& E. B. Thornton (1981). Field Observations of Surf Beat. Journal of Geophysical Research 86, pp. 6451-6466.

Holland, K. T., R. A. Holman (1999). Wavenumber-frequency structure of infragravity swash motions. Journal of Geophysical Research 104, pp. 13,479-13,488.

Holland, K. T., R. A. Holman, T. C. Lippmann, J. Stanley \& N. Plant (1997). Practical Use of Video Imagery in Nearshore Oceanographic Field Studies. Journal of Oceanic Engineering 22, pp. 81-92.

Holman, R. A., A. H. Sallenger Jr, T. C. Lippmann \& J. W. Haines (1993). The Application of Video Image Processing to the Study of Nearshore Processes. Oceanography 6, pp. 78-85.

Jenkins, G. M. \& D. G. Watts (1968). Spectral analysis and its applications. Holden-Day, San Francisco, USA.

Knaus, J. A. (1978). Introduction to Physical Oceanography, Prentice Hall, Englewood Cliffs, USA.

Lippmann, T. C. \& R. A. Holman (1991). Phase speed and angle of breaking waves measured with video techniques. In: Proc. Coastal Sediments '91, New York: ASCE, pp. 542-556.

Lippman, T. C. and R. A. Holman. 1989. Quantification of sand bar morphology: A video technique based on wave dissipation. Journal of Geophysical Research, 94(C1):995-1101.

Reniers, A. J. H. M. (1999). Longshore current dynamics. PhD. Thesis Delft University of Technology, Department of Civil Engineering and Geosciences.

Stockdon, H. F. \& R. A. Holman (2000). Estimation of wave speed and nearshore bathymetry from video imagery. Journal of Geophysical Research 105, pp. 22,015-22,033. 
Van Enckevort, I. M. J. (2001). Daily to yearly nearshore bar behaviour PhD. Thesis Utrecht University.

Van Rijn, L. C., B. G. Ruessink \& J. P. M. Mulder (2002). Coast3D - Egmond. The behaviour of a straight sandy coast on the time scale of storms and seasons. EC MAST Project No. MAS3-CT97-0086. 


\section{A Argus stations worldwide}

\begin{tabular}{|l|l|l|}
\hline Site & Country & Time of placement \\
\hline Yaquina Head, Oregon & USA & 1992 \\
\hline Duck, North Carolina & USA & 1993 \\
\hline La Jolla Shores Beach, San Diego, California & USA & 1994 \\
\hline Waimea Bay, Hawaii & USA & 1995 \\
\hline Noordwijk & The Netherlands & 1995 \\
\hline Palm Beach & Australia & 1996 \\
\hline Perranporth, England & UK & 1996 \\
\hline Coast 3D, Egmond aan Zee & The Netherlands & 1997 \\
\hline Muriwai Beach & New Zealand & 1998 \\
\hline Teignmouth, England & UK & 1999 \\
\hline Jan van Speijk lighthouse, Egmond aan Zee & The Netherlands & 1999 \\
\hline Goldcoast & Australia & 1999 \\
\hline Miyazaki & Japan & 2001 \\
\hline Torre Mapfre, Barcelona & Spain & 2001 \\
\hline Lake Worth & USA & 2001 \\
\hline Tweed Project - Coolangatta & Australia & 2002 \\
\hline Tweed Project - Duranbah & Australia & 2002 \\
\hline Tweed Project - Kirra & Australia & 2002 \\
\hline Tweed Project - Rainbow & Australia & 2002 \\
\hline El Puntal, Santander & Spain & 2002 \\
\hline Carchuna, Andalucia & Spain & 2002 \\
\hline Wamberal lagoon & Australia & 2002 \\
\hline Lido di Dante & Italy & 2003 \\
\hline Homer, Alaska & USA & 2003 \\
\hline Trafalgar & Spain & 2003 \\
\hline & & \\
\hline
\end{tabular}




\section{B Geo-referencing of oblique video data}

Standard photogrammetric procedures (Holland et al., 1997) enable the transformation from real world coordinates $(x, y, z)$ to image coordinates $(u, v)$ on the basis of the collinearity equations (Eq. (1.1) and (1.2)) .The latter contain 11 coefficients $\mathrm{L}_{1}-\mathrm{L}_{11}$, which are described as

$$
\begin{aligned}
& L=-\left(x_{c} m_{31}+y_{c} m_{32}+z_{c} m_{33}\right) \\
& L_{1}=\frac{\left(u_{0} m_{31}+f m_{11}\right)}{\left(\lambda_{u} L\right)} \\
& L_{2}=\frac{\left(u_{0} m_{32}+f m_{12}\right)}{\left(\lambda_{u} L\right)} \\
& L_{3}=\frac{\left(u_{0} m_{33}+f m_{13}\right)}{\left(\lambda_{u} L\right)} \\
& L_{4}=-\left(L_{1} x_{c}+L_{2} y_{c}+L_{3} z_{c}\right) \\
& L_{5}=\frac{\left(v_{0} m_{31}+f m_{21}\right)}{\left(\lambda_{v} L\right)} \\
& L_{6}=\frac{\left(v_{0} m_{32}+f m_{22}\right)}{\left(\lambda_{v} L\right)} \\
& L_{7}=\frac{\left(v_{0} m_{33}+f m_{23}\right)}{\left(\lambda_{v} L\right)} \\
& L_{8}=-\left(L_{5} x_{c}+L_{6} y_{c}+L_{7} z_{c}\right) \\
& L_{9}=\frac{m_{31}}{L} \\
& L_{10}=\frac{m_{32}}{L} \\
& L_{11}=\frac{m_{33}}{L}
\end{aligned}
$$

In Eq. $(2.1),(x, y, z)$ are the camera $x y z$-coordinates, $\left(u_{0}, v_{0}\right)$ are the image center $u v$ coordinates, $f$ is the effective focal length and $\lambda_{\mathrm{u}}$ and $\lambda_{\mathrm{v}}$ are the horizontal and vertical scale factors. The $m$-coefficients describe the successive rotations around the azimuth $\phi$, tilt $\tau$ and roll $\sigma$, 


$$
\begin{aligned}
& m_{11}=\cos \phi \cos \sigma+\sin \phi \cos \tau \sin \sigma \\
& m_{12}=-\sin \phi \cos \sigma+\cos \phi \cos \tau \sin \sigma \\
& m_{13}=\sin \tau \sin \sigma \\
& m_{21}=-\cos \phi \sin \sigma+\sin \phi \cos \tau \cos \sigma \\
& m_{22}=\sin \phi \sin \sigma+\cos \phi \cos \tau \cos \sigma \\
& m_{23}=\sin \tau \cos \sigma \\
& m_{31}=\sin \phi \sin \tau \\
& m_{32}=\cos \phi \sin \tau \\
& m_{33}=-\cos \tau
\end{aligned}
$$

The theoretical formulations presented here are valid for use with distortion-free lenses. Owing to the incorporation of $\lambda_{u}$ and $\lambda_{v}$, the formulations embody a correction for the slightly non-squareness of individual pixels. The latter is induced by a minor difference in sampling frequency between the camera and image acquisition hardware, which causes a minor mismatch between the number of horizontal picture elements at the camera CCD and the number of columns at the image frame buffer, where the image processing system stores the video data. 


\section{Field measurement equipment}

\section{Design and collection of video data files}

Stanley and Holman of the Coastal Imaging Lab, Oregon State University, have developed a toolbox for the design of pixel arrays that works with Matlab. (see Attachment D) In the design file three different types of setup arrangements can be defined, i.e. an alpha setup used for wave period and direction analysis, a bathy setup used for the analysis of the bathymetry via wave celerity and a vbar setup used for the longshore or cross-shore surface currents analysis, depending on the positioning of the arrays. Each setup arrangement consists of one or more pixel arrays, although it is advisable to define only one array per setup arrangement, because this will be easier to process in the analysis. The arrays consist of points that are defined in xyz space. These co-ordinates are transformed to uv space by a tool from the pixel toolbox, so that they can be sent to the obliquely viewing cameras at the site for collection. The collection is scheduled with the PIXScheduleCollect command, which specifies the cameras that are used in the collection, the number of samples that are taken in time and the frequency of the collection. While a collection is scheduled, three files are generated, i.e. a .mat, a .pix and a .sched file. The two last files are used for the collection. The .mat file is used in the analysis of the data.

The collected data are transferred as a .Z file via phone line to a base computer, from which data can be collected for further analysis. The $\mathrm{Z}$ file first has to be extracted to a ras file to enable this. The data consist of pixel intensities for the specified pixel arrays collected with the specified frequency for the specified number of points in time. This is a very large collection of pixel intensities from which the desired data can be sampled by specifying the array of interest when loading the data. Using the command loadStack returns the data for the specified array, using the .mat file that was generated when the collection was scheduled. The pixels are collected in uv co-ordinates that are transformed to xyz coordinates using the pixel analysis tools. The result is a collection of pixel intensities in two dimensions, i.e. alongshore position along the array $(\mathrm{y})$ and time ( $\mathrm{t}$ ), which shows a time stack image when plotted. This collection of pixel intensities will be called $\mathrm{I}(\mathrm{y}, \mathrm{t} ; \mathrm{x})$ in the remainder of this study.

\section{Ground truth measurements}

In the field work carried out in the framework of the present study, stand alone frames are used when obtaining ground truth data. Tripod frames are used to which an electronics container is attached (see Figure 7-1). The measuring devices are fixed to the frames. Flow velocities are measured using an Electro Magnetic Flow meter (EMF), which is shown in Figure 7-2. The frames are made heavier with three peaces of lead, attached to each leg of the frame (see Figure 7-3). For safety, an anchor is fastened to the frames (see Figure 7-4), so that in case of extreme weather condtions, the frames will stay in place. Each day, the frame is taken from the water and the measured data are read out from the data logger using a portable computer. After reading out the data, a new measuring program is fed to the data 
logger. After that, the frame is put back at the waterline again, and the tide will come up to cover the frame with water, so that new measurements can be made.

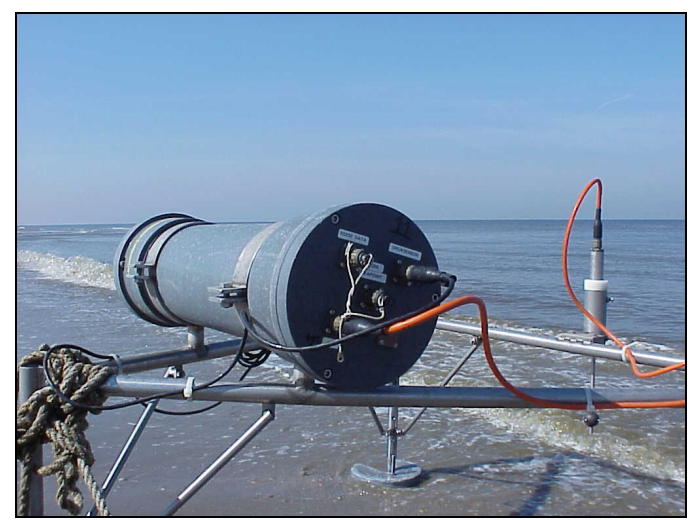

Figure 7-1 Electronics container on a measuring frame

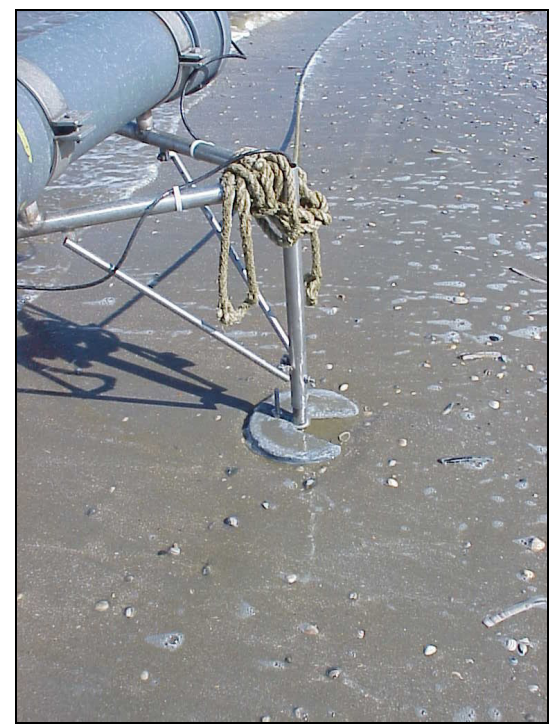

Figure 7-3 Leaden plate attached to the foot of a frameleg

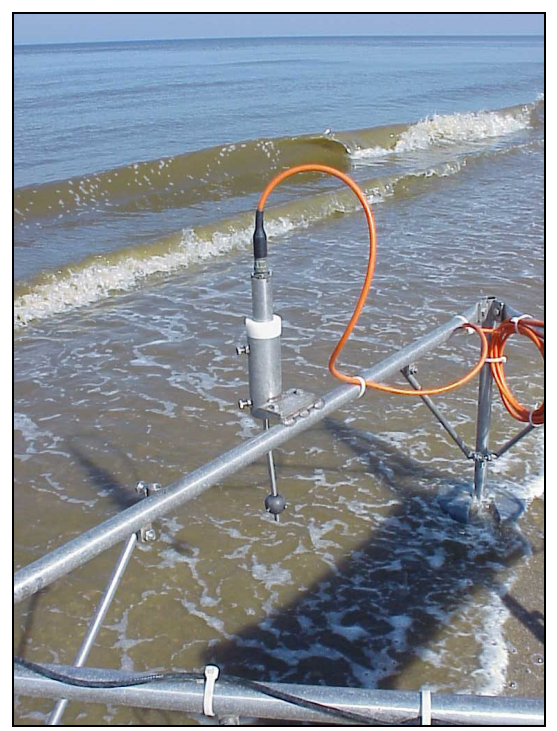

Figure 7-2 EMF current meter attached to the frame

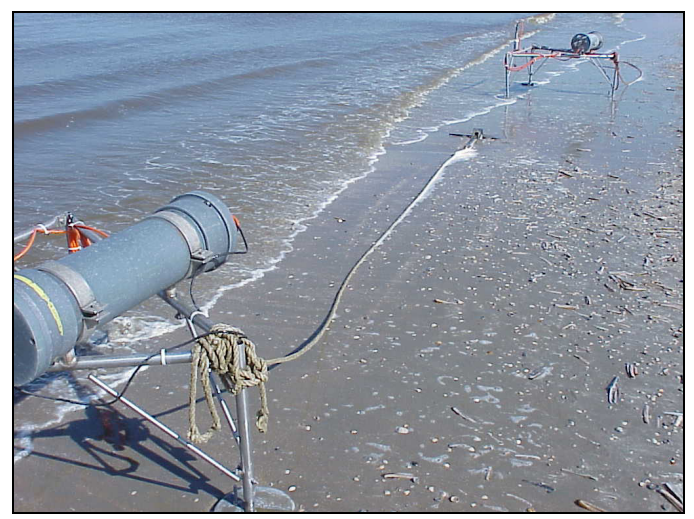

Figure 7-4 Anchor to secure the frame position 


\title{
Argus PIXel Toolbox
}

\author{
John Stanley, Rob Holman \\ Coastal Imaging Lab, Oregon State University
}

\section{Concept}

The Argus PIXel toolbox is based on the same concept that any field experiment is. Packages of instruments are deployed, where each instrument may have one or more sensors. The same ideas appear in this toolbox. Individual picp stanley/Xpdefaults . xel locations (sensors) are grouped into instruments, which are further grouped into packages. It is this package which is then deployed into the field for collection of data.

Functions are also included to provide the basis of an instrument database, although final details of this database have not been implemented.

\section{Usage}

The functions will be described in the same sequence as they might be used to create a package. Unlike normal Matlab help, the functions will be presented with correct capitalization.

\section{$2.1 \quad$ PIXForget}

PIXForget is used to clear any uncommitted instruments or packages from the workspace. This is accomplished by reloading the data for the station being worked on. If there is no stored data, this effectively removes all information about packages and instruments previously created. E.g.,

\section{PIXForget;}




\subsection{PIXSetStation}

PIXSetStation indicates which station these packages will be used at. This is the station name as found in the data files. This information is important so that the correct camera information can be retrieved later. E.g.,

PIXSetStation('tinmuth');

\subsection{PIXCreateInstrument}

While packages can be built from the top down (i.e, create the package, add instruments, and then add pixels to the instruments), it is easier to create them from the "middle out". By creating the instruments first, you can then use one call to create a package which uses them all.

The function to create an instrument is PIXCreateInstrument. Each instrument is given a name (which can be used when processing the produced stack data to retrieve the included pixels by name), a type, and optional flags that control the conversion of $\mathrm{x}, \mathrm{y}, \mathrm{z}$ real-world coordinates into $\mathrm{U}, \mathrm{V}$ image coordinates. These flags will be described later. The function returns an id, which is used with other functions to refer to this instrument. E.g.,

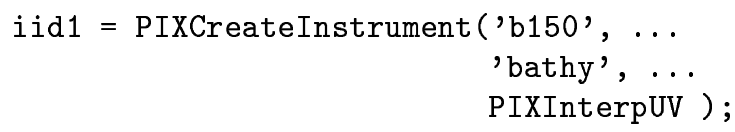

\subsubsection{Instrument Naming and Typing Convention}

The toolbox puts no limit on the names or types of instruments you may create. These strings are for your use. However, in the interests of global harmony and understanding, we will share the convention that is being adopted by the CIL for names and types.

\begin{tabular}{|c|c|c|}
\hline Type & Use & Name prefix \\
\hline \hline 'alpha' & wave period and direction array & $\mathrm{a}$ \\
\hline 'bathy' & bathymetry via wave celerity & $\mathrm{b}$ \\
\hline 'vbar' & surface currents & $\mathrm{v}$ \\
\hline 'runup' & wave runup transect & $\mathrm{r}$ \\
\hline
\end{tabular}

Names are based on the type of instrument, and include an indication of location. The names are created by appending the location to the prefix for the type. For example, a 'bathy' instrument that samples a line near $\mathrm{y}=150 \mathrm{~m}$ would be named 'b150'. A 'vbar' instrument sampling at $\mathrm{x}=300$ and $\mathrm{y}=-200$ would have a name 'v300.-200'. 
These names are being used in the CIL file naming system when results from stack processing are stored. The results from a bathy instrument at $y=304$ at the Scripps site would be stored in a file named:

1026948842.Wed.Jul.17_23_34_02.GMT.2002.argus04.b304.txt

and a plot of the data from this file would appear in:

1026948842.Wed.Jul.17_23_34_02.GMT.2002.argus04.b304.jpg

And it would look like this:

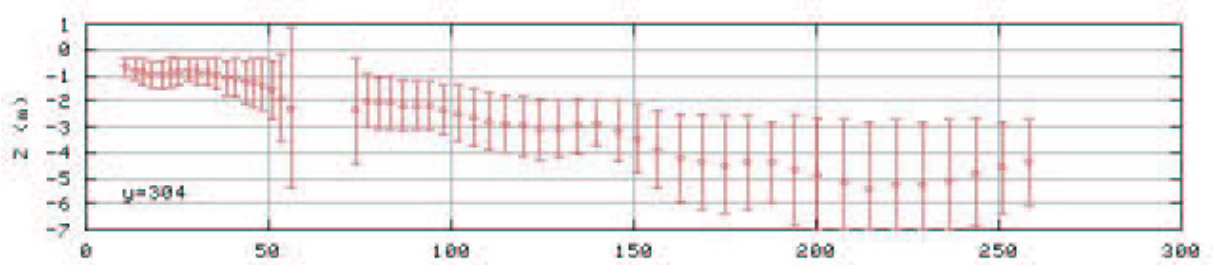

\subsection{Adding coordinates to an instrument}

There are three ways of adding real-world coordinates to an instrument. You can add individual $\mathrm{x}, \mathrm{y}, \mathrm{z}$ triples (or arrays of them), you can define a line in $\mathrm{x}, \mathrm{y}, \mathrm{z}$ space, or you can define a regularly-spaced two-dimensional set of $\mathrm{x}, \mathrm{y}, \mathrm{z}$ points (called a 'Matrix'). Notice that these are real-world coordinates, in meters, in the station coordinate system.

\subsubsection{PIXAddPoints}

To add individual points, or arrays of points, to an instrument, use the function PIXAddPoints. The parameters to this function are the instrument id, an array of $\mathrm{x}$, an array of $\mathrm{y}$, and an array of $\mathrm{z}$, and an optional array of names for each point. The arrays must be of the same length. The function returns an id for this set of coordinates, which you may typically ignore.

For example,

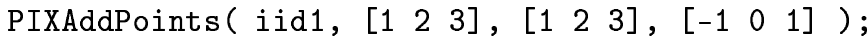

adds the three points $(1,2,-1),(2,2,0)$, and $(3,3,1)$ to the instrument created above. 


\subsubsection{PIXAddLine}

To add a line of regularly spaced coordinates to an instrument, use the function PIXAddLine. This function requires the instrument id, the $\mathrm{x}$ and $\mathrm{y}$ coordinates of the ends of the line, the $\mathrm{z}$ value for the line, the spacing, and an optional name for the line. E.g.,

PIXAddLine ( $\operatorname{idd} 1,0,0,100,0,-1,2$ );

creates a line of coordinates from $(0,0)$ to $(100,0)$ at an elevation of -1 , every 2 meters and adds it to the instrument created above.

\subsubsection{PIXAddMatrix}

A 'matrix' is a regularly-spaced two-dimensional set of coordinates. To add a matrix to an instrument, you need to provide the instrument id, the corners of the real-world rectangle, the elevation, and the $\mathrm{x}$ and $\mathrm{y}$ spacings. E.g.,

PIXAddMatrix ( iid1, 0, 0, 100, 100, -1, 1, 2);

creates a set of points from $(0,0)$ to $(100,100)$ with a point every $1 \mathrm{~m}$ in $\mathrm{x}$ and every $2 \mathrm{~m}$ in $\mathrm{y}$. The elevation of these points is set to -1 .

\subsection{PIXCreatePackage}

Once you have instruments with coordinates of pixel sensors, you can easily create a package. The function PIXCreatePackage takes a string which names the package, and an array of previously created instrument ids. You can also provide an array of names for the instruments if you wish to override the names previously given to them in the PIXCreateInstrument call. This function returns an id which is used in following functions to build and schedule the package. E.g.,

pid=PIXCreatePackage ( 'tinmuth', [iid1 iid2 iid3] );

creates a package consisting of the three instruments with the ids iid1, iid2, and iid3.

\subsection{Instrument Flags}

Now that we've described what the coordinates are, it is time to explain how they are used in creating the UV pixel coordinates when the data are actually collected. There are three flags that can be used in the PIXCreateInstrument call. These flags apply to all coordinates that make up the instrument. Note that you can create a package with instruments that have different flag values. 


\subsubsection{PIXFixedZ}

This flag instructs the toolbox to use the $\mathrm{z}$ value initially stored with the instrument instead of the tidal $\mathrm{z}$ value that will be provided when the package is scheduled. This is useful when you are defining an instrument that will sample a non-water-suface point. For example, if you are sampling the visual intensity of one of the ground-based GCPs, you do not want the $\mathrm{z}$ value to fluctuate with the incoming or outgoing tides.

\subsubsection{PIXFixedXY}

When an $\mathrm{x}, \mathrm{y}, \mathrm{z}$ coordinate is converted to an image UV coordinate, normally the UV value is determined from the $\mathrm{x}, \mathrm{y}$, and $\mathrm{z}$, then truncated to an integer, and the $\mathrm{x}, \mathrm{y}$, and $\mathrm{z}$ back-calculated for that UV integer value. (This is because the sample program at the remote end can only sample integer pixel locations.) This simplifies the process greatly if you don't care exactly where the pixels come from, as long as they come from somewhere close to the specified location. E.g., a wave directional array where the processing routine can use arbitrary $\mathrm{x}, \mathrm{y}$ locations.

When you do not want this conversion to take place, you can specify PIXFixedXY as one of the flags to the PIXCreateInstrument function. The collection routine will still convert the UV coordinate to integer prior to collection, but the back-calculation will not take place. This flag is not commonly used.

\subsubsection{PIXInterpUV}

As was previously noted, the collection routine that actually samples the video data works only with integer UV locations. This causes artifacts to appear in, e.g., linear arrays of pixels, as the line steps from one $U$ to the next (in a nearlyvertical line), or one $\mathrm{V}$ to the next in a nearly-horizontal line. This effect can be minimized by using the four surrounding pixels and doing a weighted-average to simulate non-integer pixel locations. That's what the PIXInterpUV flag tells the toolbox to do.

When you use the PIXInterpUV flag, the integer location of the four pixels surrounding the desired point will be collected, and the PIXInterp function can then be used on the returned data to do the weighted-average interpolation.

Because this flag allows non-integer UV pairs to be collected, the back-calculation of $\mathrm{x}$ and $\mathrm{y}$ are not performed. In effect, this flag supercedes the need for PIXFixedXY.

\subsubsection{Using Multiple Flags}

The flag values may either be added or 'or'd to create the final flag value. E.g., 


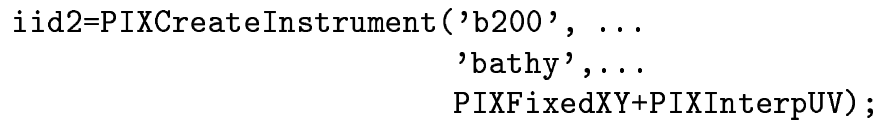

\subsection{PIXBuildCollect}

Now that you have created a package with instruments that have defined xyz coordinates, you can build the collection. This is the step that actually expands the coordinates you have defined, converts the real-world points to UV coordinates, tags those with the instrument names, and does almost all the other work required prior to actually collecting the data.

The function requires the package id, the epoch time intended for the collection, the tidal elevation, and a hint for the priority of cameras. It returns a structure that contains all the data about the package, the coordinates, the cameras, the pixels; in other words, just about everything. E.g.,

$$
\left.r=\text { PIXBuildCollect ( pid, epoch, tide, [ } \begin{array}{lllll}
2 & 3 & 1 & 4 & 5
\end{array}\right] \text { ); }
$$

This function will build the collection structure ' $r$ ' using the package with id 'pid', at epoch time 'epoch', with a tidal z of 'tide', with camera 2 given highest priority and camera 5 the lowest. Remember, however, that any instrument that has been created with the PIXFixedZ flag will not use the tidal $\mathrm{z}$ value here, but will use the $\mathrm{z}$ values of the points, lines or matrices that are part of that instrument.

\subsubsection{What is "priority"?}

Camera views almost always overlap. When they do, there will be two cameras that 'see' certain $\mathrm{x}, \mathrm{y}, \mathrm{z}$ locations. How should the software decide which camera to use to collect any points in those overlapping regions? It uses the priority that you give in the call to PIXBuildCollect.

In the example above, the cameras were listed explicitely. Any points that appear in both cameras 2 and 4 will be collected using camera 2, since it appears before camera 4 in the list. You can specify the string 'fov' to give cameras priority by field of view, which means that any zoomed-in camera has priority over one that is not zoomed-in. This is the default if only three parameters are passed to PIXBuildCollect.

If you provide an empty array (' [') for the priority, the priority will be camera number increasing. I.e., for a station with 5 cameras $1-5$, the priority [] will equate to $\left[\begin{array}{lllll}1 & 2 & 3 & 4 & 5\end{array}\right]$. 


\subsection{2 "I have points left after assigning them to all cameras."}

You may sometimes see this message appear when you build a collection. It means, simply, that some of the $\mathrm{x}, \mathrm{y}, \mathrm{z}$ coordinants you have defined in the instruments did not appear in any of the cameras available. This may happen for any number of reasons. Coordinates near the edges of an image may move outside camera range because the tidal elevation changes. You may have deliberately designed a transect (runup or bathymetry line, e.g.) so that it always extends off the edge of the image. Or you may have accidentally created such a line.

If you see this message and you don't think any of your points should be outside camera range, you should plot the UV points for each camera on an image from that camera to see what might be extending off the edge. See the section describing the 'r' structure for information on how to find the UV pixel coordinates for a camera.

\subsection{Scheduling the Collection}

Even though you have specified a time for the collection to take place in PIXBuildCollect, you have not yet scheduled the collection to take place. This has not yet happened for several reasons. First, PIXBuildCollect allows the user to experiment with different instruments and settings prior to actually causing any collection to take place. Second, it is possible to create packages that are timeindependent, so even though a time has been specified, it may not be the actual time the collection will be performed. And finally, it is possible to create a collection that has pixels in more than two cameras while the actual collection software at the Argus station can process only two at a time.

To actually schedule a collection that has been built, use the function PIXScheduleCollect. This function converts the 'r' collection structure into pixel lists and the commands needed by the remote station to actually do the collection. The only remaining bits of information required are the number of samples to be collected and the frequency.

$$
\text { PIXScheduleCollect ( [3 4], 1024, 2, r ); }
$$

This call schedules a collection using cameras 3 and 4 , for 1024 samples, at 2 Hertz, using the struct built by PIXBuildCollect. Keep in mind that the maximum frequency for two cameras is $2 \mathrm{~Hz}$, because the software does remember and will beep at you if you exceed that rate. It will also not allow more than 2 cameras to be collected at the same time.

The files produced by PIXScheduleCollect are created in a 'temp' directory underneath the current working directory. E.g., if you are in your home directory $(\sim)$, you will find three or four output files in $\sim /$ temp. These will all start with 
the epoch time for the collection. The pixel lists will have the extension '.pix'. The commands to the collection software are in the file with the extension '.sched'. The '.mat' file is a copy of the collection struct, which will be required for processing the stack data once it has been recovered from the station.

At this point, the .pix and .sched files need to be transferred to the remote station. An automated system has not yet been produced for this since almost every station requires a different means of transporting them. The .mat file does not need to be sent to the Argus station, but it does need to be copied to a location so that the stack processing routines know how to find it. Currently, this is the directory 'collects' located under the station data archive directory. E.g., '/ftp/pub/tinmuth/collects'.

\section{A Sample Program}

This is a sample program demonstrating the use of the PIXel toolbox.

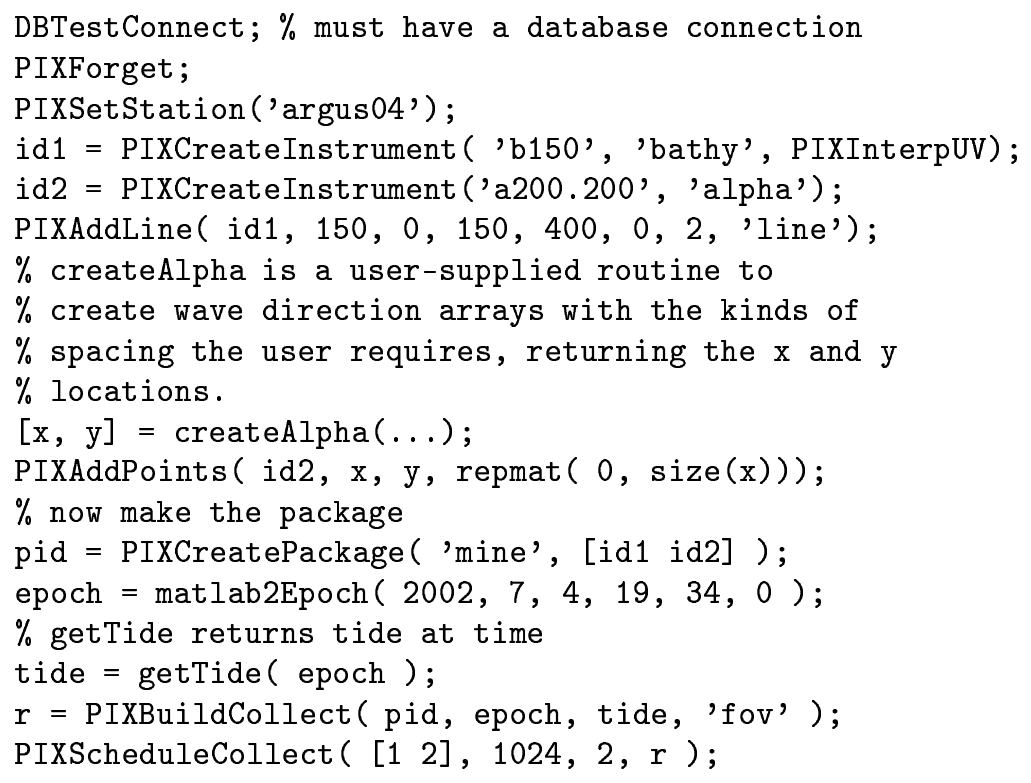

\subsection{What is this ' $r$ '?}

A good question. It is sometimes referred to as the "collection structure". Another good question is why it is called 'r'. It's called ' $r$ ' for the same reason that the SGI data collection program is called ' $m$ '. ${ }^{1}$

\footnotetext{
${ }^{1}$ Because John wanted a short name to use while debugging the code during initial testing and never changed it.
} 
What is it? It is a structure of structures that contains almost everything about the stack collection. This is an example from the Scripps station:

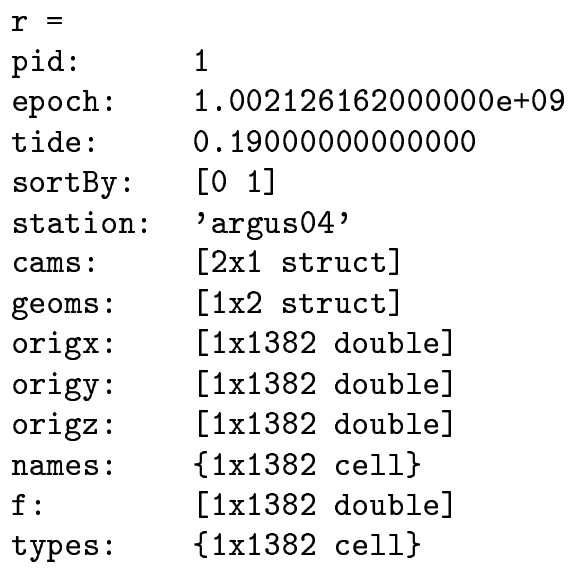

Most of the entries are self-explanatory. The 'cams' array is an array of camera structures (as found in the database) with data specific to the camera added. The raw $\mathrm{U}, \mathrm{V}$, name and flag data are included, as are the final U, V, names and types for each UV pair collected for that camera. The cams struct also contains the $\mathrm{X}, \mathrm{Y}$, and $\mathrm{Z}$ data for the raw UV coordinates. If these arrays are empty, then there were no UV pairs calculated for this camera. Note carefully that the cameras are NOT guaranteed to be in camera number order. I.e., r.cams(1) is not guaranteed to be camera 1 .

\section{Processing stack data}

Because the PIXel toolbox has used a standard set of names for the pixel list files and matlab collection struct storage, it can extract the pixel list name from the stack datafile itself and locate the information about the instruments contained therein. This makes pixel data processing much simpler.

\subsection{Showing PIXel instruments}

A convenient routine for showing the location of pixel instruments on a camera image has been created. showPIXInstruments will take a stack file name and produce an image from the corresponding snapshot with the instrument locations plotted. This image will be in oblique mode. E.g.,

showPIXInstruments ('1000000000.xxx') ; 


\section{2 loadStack}

"loadStack" is the Matlab interface into the Sun rasterfile format stack data. That function knows the encoding for the data contained in the rasterfile, which is much more than just the pixel values. Also included in the stack file are the UV coordinates themselves, the sample rate, the name of the pixel file, and time information about each sample.

There are several functional forms of loadStack. The simplest form is:

$$
\text { params = loadStack ( FILENAME, 'info' ); }
$$

This call returns the parameter structure for the requested stack. This structure contains information about how the stack was collected, with the pixel file (also known as the "Area Of Interest" or AOI file). This filename is used to find the collection structure file.

Here is the parameter struct from a typical stack:

$\begin{array}{ll}\mathrm{p}= & \\ \text { magic: } & 1.5041 \mathrm{e}+09 \\ \text { width: } & 1534 \\ \text { height: } & 2056 \\ \text { pixels : } & 1526 \\ \text { when: } & 1.0263 \mathrm{e}+09 \\ \text { camera: } & 1 \\ \text { version: } & 4 \\ \text { increment: } & 15 \\ \text { isColor: } & 0 \\ \text { lines: } & 2048 \\ \text { where: } & \text { 'argus04, } \\ \text { ust: } & 6.4871 \mathrm{e}+06 \\ \text { aoifile: } & , 1003508562 . \mathrm{c} 1 . \mathrm{pix}, \\ \text { syncTime: } & 1.0263 \mathrm{e}+09 \\ \text { U: } & {[1526 \times 1 \text { double] }} \\ \text { V: } & {[1526 \times 1 \text { double] }} \\ \text { order: } & {[1526 \times 1 \text { double] }}\end{array}$

The important values in that struct are the 'pixels' (number of UV's per line), the 'lines' (number of images sampled), the 'increment' ( $30 /$ increment is the sample frequency), and the aoifile.

The second most useful version of loadStack is:

$[\mathrm{p}$, epoch, msc, data $]=$ loadStack ( filename, UV ); 
This returns the parameter struct 'p', an array of epoch times (one per stack line), an array of "media system counters" (basically, a frame counter), and a 2-D array of pixel intensities. each column in "data" corresponds to one UV coordinate as listed in the $\mathrm{Nx} 2 \mathrm{UV}$ array.

Further information on loadStack can be found by using "help loadStack".

\subsection{PIXGetRFromAOIName}

This function takes the station name (e.g., 'argus04') and the AOI filename (from loadStack), and finds the stored collection structure that produced that AOI file. Note that multiple stacks can be produced from the same AOI file.

Once you have retrieved the 'r' structure for a stack by getting the AOI filename from the stack and then the $\mathrm{R}$ for that AOI file, you can use the names in that struct to find UV coordinates, and pass those UV values to loadStack to retrieve your data.

While creating stack lists is now much simpler, there is a tradeoff. ${ }^{2}$ You must be careful to note that the order of the pixels in the pixel list may no longer match the order you expect them to appear in. It is quite likely that a runup transect you have defined as one instrument will be interspersed in the stack with pixels from other instruments. This is a side-effect of collecting only the unique UV pixels in the full list, which is a side-effect of allowing people to define a line of pixels with a spacing of $1 \mathrm{~cm}$. The goal is to reduce the size of the stack by eliminating duplicate columns. The result is that you must keep track of the actual order of the UV pixels. Once you have selected all the UV coordinates that coorespond to a runup transect ('r123', for example), a simple sort based on $\mathrm{V}$ will most likely return the array of pixel values to the sequence you would expect.

The function 'showPIXInstruments' contains an example of the use of loadStack and PIXGetRFromAOIName.

\subsection{PIXFindUVByName}

If you know the name of the instrument you are seeking, this function will retrieve the $\mathrm{U}$ and $\mathrm{V}$ coordinates for those instruments. It will also optionally retrieve the raw $\mathrm{U}$ and $\mathrm{V}$ and the $\mathrm{x}, \mathrm{y}, \mathrm{z}$ coordinates. The $\mathrm{U}$ and $\mathrm{V}$ coordinates can then be passed to loadStack to retrieve the intensity values.

What is the difference between the $\mathrm{U}$ and $\mathrm{V}$, and the raw $\mathrm{U}$ and $\mathrm{V}$, you ask? The raw version is the double value, while the $\mathrm{U}$ and $\mathrm{V}$ are the integers. The integer values are what the remote system actually collects. The raw values are the idea coordinates.

\footnotetext{
${ }^{2}$ There is always a tradeoff.
} 


\subsection{PIXInterp}

"PIXInterp" performs a weighted average of the sampled pixel intensity to reduce the aliasing ('stairstep') problem mentioned earlier. It takes the intensity data produced by loadStack and the rawUV information from PIXFindUVByName.

\subsection{A Special Note on Interpolation}

You probably recall the previous discussion of the PIXInterpUV collection flag. This flag tells the PIXel toolbox to sample the four pixels surrounding the actual physical location. Thus, when you retrieve the UV coordinates for an instrument that has this flag set, you will get four times as many UV coordinates as you would expect. The function PIXInterp takes the quadruplicate data set and reduces it to the interpolated values. In order to do this, it requires the floating point UV coordinates (rawUV mentioned above).

This function requires that NO sorting of the coordinants take place prior to interpolation. It expects the intensity data it works with to be in a specific order, and any sorting of the data by UV or xyz location will destroy this order. Sort the resulting data only after PIXInterp.

\section{$5 \quad$ Further Routines}

This toolbox is in the user-driven stage. The functions necessary to create pixel lists have been written, and basic data access routines are available. Now I need users to determine what else they need; what other functions should be and would be useful. Those will be added. There are other routines in the toolbox, most of which are used internally. The function of the remainder may be ascertained from the souce. 


\section{E Data selection Egmond and Noordwijk experiment}

\section{Egmond (video and ground truth file, gray=not useful)}

\begin{tabular}{|c|c|c|}
\hline & & \\
\hline D:ICoastview2002legmond_noQCVInst811035354721.Wed.Oct.23_06_32_01.GMT.2002.egmond.c1v10.-380.mat & CD & \\
\hline D:ICoastview2002legmond_noQCVInst8\1035358322.Wed.Oct.23_07_32_02.GMT.2002.egmond.c1v10.-380.mat & $C D$ & 7089 \\
\hline D:ICoastview2002legmond_noQCl|nst8\1035361921.Wed.Oct.23 08 32_01.GMT.2002.egmond.c1v10.-380.mat & $C D$ & 7090 \\
\hline D:ICoastview2002legmond_noQCVInst811035365521.Wed.Oct.23_09_32_01.GMT.2002.egmond.c1v10.-380.mat & $C D$ & 7091 \\
\hline D:ICoastview2002legmond_noQCVInst8\1035369122.Wed.Oct.23_10_32_02.GMT.2002.egmond.c1v10.-380.mat & $C D$ & 7092 \\
\hline D:ICoastview2002legmond_noQCVInst811035372722.Wed.Oct.23_11_32_02.GMT.2002.egmond.c1v10.-380.mat & $\mathrm{CD}$ & 7093 \\
\hline D:ICoastview2002legmond_noQCVInst811035376321.Wed.Oct.23_12_32_01.GMT.2002.egmond.c1v10.-380.mat & $\mathrm{CD}$ & 7094 \\
\hline D:ICoastview2002legmond_noQCVInst811035379922.Wed.Oct.23_13_32_02.GMT.2002.egmond.c1v10.-380.mat & $\mathrm{CD}$ & 7095 \\
\hline D:ICoastview2002legmond_noQCVInst8\1035383522.Wed.Oct.23_14_32_02.GMT.2002.egmond.c1v10.-380.mat & $C D$ & 7096 \\
\hline D:ICoastview2002legmond_noQCVInst811035387122.Wed.Oct.23_15_32_02.GMT.2002.egmond.c1v10.-380.mat & $\mathrm{CD}$ & 7097 \\
\hline D:ICoastview2002legmond_noQCVInst811035390722.Wed.Oct.23_16_32_02.GMT.2002.egmond.c1v10.-380.mat & $\mathrm{CD}$ & 7098 \\
\hline D:ICoastview2002legmond_noQCVInst8|1035441122.Thu.Oct.24_06_32_02.GMT.2002.egmond.c1v10.-380.mat & $\overline{C D}$ & 7112 \\
\hline D:ICoastview2002legmond_noQCVInst8\1035444721.Thu.Oct.24_07_32_01.GMT.2002.egmond.c1v10.-380.mat & $\mathrm{CD}$ & 7113 \\
\hline D:ICoastview2002legmond_noQCVInst8l1035448322.Thu.Oct.24_08_32_02.GMT.2002.egmond.c1v10.-380.mat & $C D$ & 7114 \\
\hline D:ICoastview2002legmond_noQC $\mid$ nst8|1035451921.Thu.Oct.24_09_32_01.GMT.2002.egmond.c1v10.-380.mat & $C D$ & 7115 \\
\hline D:ICoastview2002legmond_noQC $\mid$ nst8\1035455522.Thu.Oct.24_10_32_02.GMT.2002.egmond.c1v10.-380.mat & $C D$ & 7116 \\
\hline D:ICoastview2002legmond_noQC $\mid$ nst8\1035459122.Thu.Oct.24_11_32_02.GMT.2002.egmond.c1v10.-380.mat & $\mathrm{CD}$ & 7117 \\
\hline D:ICoastview2002legmond_noQCVInst8\1035462722.Thu.Oct.24_12_32_02.GMT.2002.egmond.c1v10.-380.mat & $C D$ & 7118 \\
\hline D:ICoastview2002legmond_noQCVInst8l1035466321.Thu.Oct.24_13_32_01.GMT.2002.egmond.c1v10.-380.mat & $C D$ & 7119 \\
\hline D:ICoastview2002legmond_noQCVInst8l1035469922.Thu.Oct.24_14_32_02.GMT.2002.egmond.c1v10.-380.mat & $C D$ & 7120 \\
\hline D:ICoastview2002legmond_noQCVInst8\1035473522.Thu.Oct.24_15_32_02.GMT.2002.egmond.c1v10.-380.mat & $C D$ & 7121 \\
\hline D:ICoastview2002legmond_noQCVInst8\1035477122.Thu.Oct.24_16_32_02.GMT.2002.egmond.c1v10.-380.mat & CD & 7122 \\
\hline D:ICoastview2002legmond_noQC\Inst8|1035527522.Fri.Oct.25_06_32_02.GMT.2002.egmond.c1v10.-380.mat & $\mathrm{CD}$ & 7136 \\
\hline D:ICoastview2002legmond_noQCVInst8\1035531121.Fri.Oct.25_07_32_01.GMT.2002.egmond.c1v10.-380.mat & $C D$ & 7137 \\
\hline D:ICoastview2002legmond_noQCVInst811035534721.Fri.Oct.25_08_32_01.GMT.2002.egmond.c1v10.-380.mat & $C D$ & 7138 \\
\hline D:ICoastview2002legmond_noQCVInst811035538321.Fri.Oct.25_09_32_01.GMT.2002.egmond.c1v10.-380.mat & $C D$ & 7139 \\
\hline D:ICoastview2002legmond_noQCVInst8\1035541921.Fri.Oct.25_10_32_01.GMT.2002.egmond.c1v10.-380.mat & $C D$ & 7140 \\
\hline D:ICoastview2002legmond_noQCVInst811035545521.Fri.Oct.25_11_32_01.GMT.2002.egmond.c1v10.-380.mat & $C D$ & 7141 \\
\hline D:ICoastview2002legmond_noQCVInst811035549122.Fri.Oct.25_12_32_02.GMT.2002.egmond.c1v10.-380.mat & $C D$ & 7142 \\
\hline D:ICoastview2002legmond_noQCVInst811035552722.Fri.Oct.25_13_32_02.GMT.2002.egmond.c1v10.-380.mat & $C D$ & 7143 \\
\hline D:ICoastview2002legmond_noQCVInst8\1035556322.Fri.Oct.25_14_32_02.GMT.2002.egmond.c1v10.-380.mat & $\mathrm{CD}$ & 7144 \\
\hline D:ICoastview2002legmond_noQCVInst8\1035559922.Fri.Oct.25_15_32_02.GMT.2002.egmond.c1v10.-380.mat & $\mathrm{CD}$ & 7145 \\
\hline D:ICoastview2002legmond_noQCVInst8\1035563522.Fri.Oct.25_16_32_02.GMT.2002.egmond.c1v10.-380.mat & $\mathrm{CD}$ & 7146 \\
\hline D:ICoastview2002legmond_noQCVInst811035613922.Sat.Oct.26_06_32_02.GMT.2002.egmond.c1v10.-380.mat & $C D$ & 7160 \\
\hline D:ICoastview2002legmond_noQCVInst811035617522.Sat.Oct.26_07_32_02.GMT.2002.egmond.c1v10.-380.mat & $C D$ & 7161 \\
\hline D:ICoastview2002legmond_noQCVInst811035621122.Sat.OCt.26_08_32_02.GMT.2002.egmond.c1v10.-380.mat & $\mathrm{CD}$ & 7162 \\
\hline D:ICoastview2002legmond_noQCVInst8\1035624722.Sat.Oct.26_09_32_02.GMT.2002.egmond.c1v10.-380.mat & $C D$ & 7163 \\
\hline D:ICoastview2002legmond_noQC\Inst8\1035628321.Sat.Oct.26_10_32_01.GMT.2002.egmond.c1v10.-380.mat & $C D$ & 7164 \\
\hline D:ICoastview2002legmond_noQCVInst8\1035631922.Sat.Oct.26_11_32_02.GMT.2002.egmond.c1v10.-380.mat & $C D$ & 7165 \\
\hline D:ICoastview2002legmond_noQCVInst811035635524.Sat.OCt.26_12_32_04.GMT.2002.egmond.c1v10.-380.mat & $C D$ & 7166 \\
\hline D:ICoastview2002legmond_noQC\Inst8\1035639121.Sat.Oct.26_13_32_01.GMT.2002.egmond.c1v10.-380.mat & $C D$ & 7167 \\
\hline D:ICoastview2002legmond_noQCVInst8\1035642722.Sat.Oct.26_14_32_02.GMT.2002.egmond.c1v10.-380.mat & $C D$ & 7168 \\
\hline D:ICoastview2002legmond_noQCVInst8\1035646321.Sat.Oct.26_15_32_01.GMT.2002.egmond.c1v10.-380.mat & $C D$ & 7169 \\
\hline D:ICoastview2002legmond_noQCVInst8\1035649921.Sat.Oct.26_16_32_01.GMT.2002.egmond.c1v10.-380.mat & CD & 7170 \\
\hline D:ICoastview2002legmond_noQCVInst8l1035700322.Sun.0ct.27_06_32_02.GMT.2002.egmond.c1v10.-380.mat & $\mathrm{CD}$ & 7184 \\
\hline D:ICoastview2002legmond_noQCVInst8\1035703922.Sun.Oct.27_07_32_02.GMT.2002.egmond.c1v10.-380.mat & $\mathrm{CD}$ & 7185 \\
\hline D:ICoastview2002legmond_noQCVInst8\1035707522.Sun.Oct.27_08_32_02.GMT.2002.egmond.c1v10.-380.mat & $C D$ & 7186 \\
\hline D:ICoastview2002legmond_noQCVInst811035711122.Sun.Oct.27_09_32_02.GMT.2002.egmond.c1v10.-380.mat & $C D$ & 7187 \\
\hline D:ICoastview2002legmond_noQCVInst8|1035714721.Sun.Oct.27_10_32_01.GMT.2002.egmond.c1v10.-380.mat & $C D$ & 7188 \\
\hline D:ICoastview2002legmond_noQCVInst8l1035718322.Sun.Oct.27_11_32_02.GMT.2002.egmond.c1v10.-380.mat & $\mathrm{CD}$ & 7189 \\
\hline D:ICoastview2002legmond_noQCVInst8l1035721922.Sun.Oct.27_12_32_02.GMT.2002.egmond.c1v10.-380.mat & $\mathrm{CD}$ & 7190 \\
\hline D:ICoastview2002legmond_noQCVInst811035725522.Sun.Oct.27_13_32_02.GMT.2002.egmond.c1v10.-380.mat & $\mathrm{CD}$ & 7191 \\
\hline D:ICoastview2002legmond_noQCVInst8\1035729122.Sun.Oct.27_14_32_02.GMT.2002.egmond.c1v10.-380.mat & $\mathrm{CD}$ & 7192 \\
\hline D:ICoastview2002legmond_noQCVInst8l1035732721.Sun.Oct.27_15_32_01.GMT.2002.egmond.c1v10.-380.mat & $\mathrm{CD}$ & 7193 \\
\hline D:ICoastview2002legmond_noQCVInst811035736322.Sun.Oct.27_16_32_02.GMT.2002.egmond.c1v10.-380.mat & $\mathrm{CD}$ & 7194 \\
\hline D:ICoastview2002legmond_noQCVInst811035786722.Mon.0ct.28_06_32_02.GMT.2002.egmond.c1v10.-380.mat & CB & 7208 \\
\hline D:ICoastview2002legmond_noQCVInst8l1035790321.Mon.Oct.28_07_32_01.GMT.2002.egmond.c1v10.-380.mat & CB & 7209 \\
\hline D:ICoastview2002legmond_noQCVInst811035793921.Mon.Oct.28_08_32_01.GMT.2002.egmond.c1v10.-380.mat & $\mathrm{CB}$ & 7210 \\
\hline D:ICoastview2002legmond_noQCVInst811035797522.Mon.Oct.28_09_32_02.GMT.2002.egmond.c1v10.-380.mat & $\mathrm{CB}$ & 7211 \\
\hline D:ICoastview2002legmond_noQCVInst811035801122.Mon.Oct.28_10_32_02.GMT.2002.egmond.c1v10.-380.mat & $\mathrm{CB}$ & 7212 \\
\hline D:ICoastview2002legmond_noQCVInst8|1035804722.Mon.Oct.28_11_32_02.GMT.2002.egmond.c1v10.-380.mat & $\mathrm{CB}$ & 7213 \\
\hline D:ICoastview2002legmond_noQCVInst8|1035808321.Mon.Oct.28_12_32_01.GMT.2002.egmond.c1v10.-380.mat & $\mathrm{CB}$ & 7214 \\
\hline D:ICoastview2002legmond_noQCVnst811035811922.Mon.Oct.28_13_32_02.GMT.2002.egmond.c1v10.-380.mat & CB & 7215 \\
\hline D:ICoastview2002legmond_noQCVInst811035815522.Mon.Oct.28_14_32_02.GMT.2002.egmond.c1v10.-380.mat & CB & 7216 \\
\hline D:ICoastview2002legmond_noQCVInst8|1035819121.Mon.Oct.28_15_32_01.GMT.2002.egmond.c1v10.-380.mat & $\mathrm{CB}$ & 7217 \\
\hline D:ICoastview2002legmond_noQCVInst811035822722.Mon.Oct.28_16_32_02.GMT.2002.egmond.c1v10.-380.mat & CB & 7218 \\
\hline D:ICoastview2002legmond_noQCVInst811035873122.Tue.Oct.29_06_32_02.GMT.2002.egmond.c1v10.-380.mat & $\mathrm{CB}$ & 7232 \\
\hline D:ICoastview2002legmond_noQCVInst8\1035876722.Tue.Oct.29_07_32_02.GMT.2002.egmond.c1v10.-380.mat & $\mathrm{CB}$ & 7233 \\
\hline D:ICoastview2002legmond_noQCVInst811035880322.Tue.Oct.29_08_32_02.GMT.2002.egmond.c1v10.-380.mat & $\mathrm{CB}$ & 7234 \\
\hline D:ICoastview2002legmond_noQCVInst8\1035883921.Tue.Oct.29_09_32_01.GMT.2002.egmond.c1v10.-380.mat & CB & 7235 \\
\hline D:ICoastview2002legmond_noQC \nst8\1035887522.Tue.Oct.29_10_32_02.GMT.2002.egmond.c1v10.-380.mat & CB & 7236 \\
\hline D:ICoastview2002legmond_noQCVInst8l1035891122.Tue.Oct.29_11_32_02.GMT.2002.egmond.c1v10.-380.mat & $\mathrm{CB}$ & 7237 \\
\hline D:ICoastview2002legmond_noQCVInst8\1035894721.Tue.Oct.29_12_32_01.GMT.2002.egmond.c1v10.-380.mat & CB & 7238 \\
\hline
\end{tabular}




\begin{tabular}{|c|c|c|}
\hline D:ICoastview2002legmond_noQC\Inst8l1035898321.Tue.Oct.29_13_32_01.GMT.2002.egmond.c1v10.-380.mat & CB & 7239 \\
\hline D:ICoastview2002legmond_noQC/Inst8l1035901922.Tue.Oct.29_14_32_02.GMT.2002.egmond.c1v10.-380.mat & $\mathrm{CB}$ & 7240 \\
\hline D:ICoastview2002legmond_noQCVInst8l1035905522.Tue.Oct.29_15_32_02.GMT.2002.egmond.c1v10.-380.mat & $\mathrm{CB}$ & 7241 \\
\hline D:ICoastview2002legmond_noQC/Inst8l1035909122.Tue.Oct.29_16_32_02.GMT.2002.egmond.c1v10.-380.mat & CB & 7242 \\
\hline D:ICoastview2002legmond_noQC|Inst811035959522.Wed.Oct.30_06_32_02.GMT.2002.egmond.c1v10.-380.mat & $\mathrm{CC}$ & 7256 \\
\hline D:ICoastview2002legmond_noQC|Inst8|1035963121.Wed.Oct.30_07_32_01.GMT.2002.egmond.c1v10.-380.mat & $\mathrm{CC}$ & 7257 \\
\hline D:ICoastview2002legmond_noQClInst811035966721.Wed.Oct.30_08_32_01.GMT.2002.egmond.c1v10.-380.mat & $\mathrm{CC}$ & 7258 \\
\hline D:ICoastview2002legmond_noQCVInst811035970323.Wed.Oct.30_09_32_03.GMT.2002.egmond.c1v10.-380.mat & $\mathrm{CC}$ & 7259 \\
\hline D:ICoastview2002legmond_noQClInst811035973921.Wed.Oct.30_10_32_01.GMT.2002.egmond.c1v10.-380.mat & $\mathrm{CC}$ & 7260 \\
\hline D:ICoastview2002legmond_noQClInst811035977521.Wed.Oct.30_11_32_01.GMT.2002.egmond.c1v10.-380.mat & $\mathrm{CC}$ & 7261 \\
\hline D:ICoastview2002legmond_noQCVInst8|1035981121.Wed.Oct.30_12_32_01.GMT.2002.egmond.c1v10.-380.mat & $\mathrm{CC}$ & 7262 \\
\hline D:ICoastview2002legmond_noQCVInst8|1035984721.Wed.Oct.30_13_32_01.GMT.2002.egmond.c1v10.-380.mat & $\mathrm{CC}$ & 7263 \\
\hline D:ICoastview2002legmond_noQCVInst8|1035988321.Wed.Oct.30_14_32_01.GMT.2002.egmond.c1v10.-380.mat & $\mathrm{CC}$ & 7264 \\
\hline D:ICoastview2002legmond_noQClInst811035991921.Wed.Oct.30_15_32_01.GMT.2002.egmond.c1v10.-380.mat & $\mathrm{CC}$ & 7265 \\
\hline D:ICoastview2002legmond_noQC|Inst811035995522.Wed.Oct.30_16_32_02.GMT.2002.egmond.c1v10.-380.mat & $\mathrm{CC}$ & 7266 \\
\hline D:ICoastview2002legmond_noQC|Inst811036045922.Thu.Oct.31_06_32_02.GMT.2002.egmond.c1v10.-380.mat & CC & 7280 \\
\hline D:ICoastview2002legmond_noQCVInst8l1036049522.Thu.Oct.31_07_32_02.GMT.2002.egmond.c1v10.-380.mat & $\mathrm{CC}$ & 7281 \\
\hline D:ICoastview2002legmond_noQCVInst8l1036053122.Thu.Oct.31_08_32_02.GMT.2002.egmond.c1v10.-380.mat & $\mathrm{CC}$ & 7282 \\
\hline D:ICoastview2002legmond_noQCVInst8l1036056722.Thu.Oct.31_09_32_02.GMT.2002.egmond.c1v10.-380.mat & $\mathrm{CC}$ & 7283 \\
\hline D:ICoastview2002legmond_noQC/Inst8l1036060321.Thu.Oct.31_10_32_01.GMT.2002.egmond.c1v10.-380.mat & $\mathrm{CC}$ & 7284 \\
\hline D:ICoastview2002legmond_noQCVInst8l1036063921.Thu.Oct.31_11_32_01.GMT.2002.egmond.c1v10.-380.mat & CC & 7285 \\
\hline D:ICoastview2002legmond_noQCVInst8l1036067522.Thu.Oct.31_12_32_02.GMT.2002.egmond.c1v10.-380.mat & $\mathrm{CC}$ & 7286 \\
\hline D:ICoastview2002legmond_noQC/Inst8l1036071122.Thu.Oct.31_13_32_02.GMT.2002.egmond.c1v10.-380.mat & $\mathrm{CC}$ & 7287 \\
\hline D:ICoastview2002legmond_noQCVInst8l1036074721.Thu.Oct.31_14_32_01.GMT.2002.egmond.c1v10.-380.mat & $\mathrm{CC}$ & 7288 \\
\hline D:ICoastview2002legmond_noQC/Inst8l1036078322.Thu.Oct.31_15_32_02.GMT.2002.egmond.c1v10.-380.mat & $\mathrm{CC}$ & 7289 \\
\hline D:ICoastview2002legmond_noQCVInst8l1036081922.Thu.Oct.31_16_32_02.GMT.2002.egmond.c1v10.-380.mat & $\mathrm{CC}$ & 7290 \\
\hline \multicolumn{3}{|l|}{ Instrument 10} \\
\hline D:ICoastview2002legmond_noQC|Inst1011035354721.Wed.Oct.23_06_32_01.GMT.2002.egmond.c1v20.-380.mat & $\mathrm{CC}$ & 7088 \\
\hline D:ICoastview2002legmond_noQCVInst10l1035358322.Wed.Oct.23_07_32_02.GMT.2002.egmond.c1v20.-380.mat & $\mathrm{CC}$ & 7089 \\
\hline D:ICoastview2002legmond_noQCVInst1011035361921.Wed.Oct.23_08_32_01.GMT.2002.egmond.c1v20.-380.mat & $\mathrm{CC}$ & 7090 \\
\hline D:ICoastview2002legmond_noQCVInst1011035365521.Wed.Oct.23_09_32_01.GMT.2002.egmond.c1v20.-380.mat & $\mathrm{CC}$ & 7091 \\
\hline D:ICoastview2002legmond_noQC|Inst1011035369122.Wed.Oct.23_10_32_02.GMT.2002.egmond.c1v20.-380.mat & $\mathrm{CC}$ & 7092 \\
\hline D:ICoastview2002legmond_noQC|Inst1011035372722.Wed.Oct.23_11_32_02.GMT.2002.egmond.c1v20.-380.mat & $\mathrm{CC}$ & 7093 \\
\hline D:ICoastview2002legmond_noQC|Inst10l1035376321.Wed.Oct.23_12_32_01.GMT.2002.egmond.c1v20.-380.mat & $\mathrm{CC}$ & 7094 \\
\hline D:ICoastview2002legmond_noQC|Inst10l1035379922.Wed.Oct.23_13_32_02.GMT.2002.egmond.c1v20.-380.mat & $\mathrm{CC}$ & 7095 \\
\hline D:ICoastview2002legmond_noQC|Inst1011035383522.Wed.Oct.23_14_32_02.GMT.2002.egmond.c1v20.-380.mat & $\mathrm{CC}$ & 7096 \\
\hline D:ICoastview2002legmond_noQClInst1011035387122.Wed.Oct.23_15_32_02.GMT.2002.egmond.c1v20.-380.mat & $\mathrm{CC}$ & 7097 \\
\hline D:ICoastview2002legmond_noQC|Inst1011035390722.Wed.Oct.23_16_32_02.GMT.2002.egmond.c1v20.-380.mat & $\mathrm{CC}$ & 7098 \\
\hline D:ICoastview2002legmond_noQC|Inst10l1035441122.Thu.Oct.24_06_32_02.GMT.2002.egmond.c1v20.-380.mat & $\mathrm{CC}$ & 7112 \\
\hline D:ICoastview2002legmond_noQCVInst1011035444721.Thu.Oct.24_07_32_01.GMT.2002.egmond.c1v20.-380.mat & $\mathrm{CC}$ & 7113 \\
\hline D:ICoastview2002legmond_noQC\Inst1011035448322.Thu.Oct.24_08_32_02.GMT.2002.egmond.c1v20.-380.mat & $\mathrm{CC}$ & 7114 \\
\hline D:ICoastview2002legmond_noQC\Inst1011035451921.Thu.Oct.24_09_32_01.GMT.2002.egmond.c1v20.-380.mat & $\mathrm{CC}$ & 7115 \\
\hline D:ICoastview2002legmond_noQC\Inst1011035455522.Thu.Oct.24_10_32_02.GMT.2002.egmond.c1v20.-380.mat & $\mathrm{CC}$ & 7116 \\
\hline D:ICoastview2002legmond_noQC\Inst10l1035459122.Thu.Oct.24_11_32_02.GMT.2002.egmond.c1v20.-380.mat & $\mathrm{CC}$ & 7117 \\
\hline D:ICoastview2002legmond_noQCVInst1011035462722.Thu.Oct.24_12_32_02.GMT.2002.egmond.c1v20.-380.mat & $\mathrm{CC}$ & 7118 \\
\hline D:ICoastview2002legmond_noQC\Inst1011035466321.Thu.Oct.24_13_32_01.GMT.2002.egmond.c1v20.-380.mat & $\mathrm{CC}$ & 7119 \\
\hline D:ICoastview2002legmond_noQC\Inst1010355469922.Thu.Oct.24_14_32_02.GMT.2002.egmond.c1v20.-380.mat & CC & 7120 \\
\hline D:ICoastview2002legmond_noQC\Inst1011035473522.Thu.Oct.24_15_32_02.GMT.2002.egmond.c1v20.-380.mat & $\mathrm{CC}$ & 7121 \\
\hline D:ICoastview2002legmond_noQC\Inst1011035477122.Thu.Oct.24_16_32_02.GMT.2002.egmond.c1v20.-380.mat & $\mathrm{CC}$ & 7122 \\
\hline D:ICoastview2002legmond_noQCVInst1011035527522.Fri.Oct.25_06_32_02.GMT.2002.egmond.c1v20.-380.mat & $\mathrm{CC}$ & 7136 \\
\hline D:ICoastview2002legmond_noQCVInst10l1035531121.Fri.Oct.25_07_32_01.GMT.2002.egmond.c1v20.-380.mat & $\mathrm{CC}$ & 7137 \\
\hline D:ICoastview2002legmond_noQC Inst10l1035534721.Fri.Oct.25_08_32_01.GMT.2002.egmond.c1v20.-380.mat & $\mathrm{CC}$ & 7138 \\
\hline D:ICoastview2002legmond_noQCVInst10l1035538321.Fri.Oct.25_09_32_01.GMT.2002.egmond.c1v20.-380.mat & $\mathrm{CC}$ & 7139 \\
\hline D:ICoastview2002legmond_noQC/Inst10l1035541921.Fri.Oct.25_10_32_01.GMT.2002.egmond.c1v20.-380.mat & $\mathrm{CC}$ & 7140 \\
\hline D:ICoastview2002legmond_noQCVInst10l1035545521.Fri.Oct.25_11_32_01.GMT.2002.egmond.c1v20.-380.mat & $\mathrm{CC}$ & 7141 \\
\hline D:ICoastview2002legmond_noQCVInst10l1035549122.Fri.Oct.25_12_32_02.GMT.2002.egmond.c1v20.-380.mat & $\mathrm{CC}$ & 7142 \\
\hline D:ICoastview2002legmond_noQCVInst10l1035552722.Fri.Oct.25_13_32_02.GMT.2002.egmond.c1v20.-380.mat & CC & 7143 \\
\hline D:ICoastview2002legmond_noQCVInst10l1035556322.Fri.Oct.25_14_32_02.GMT.2002.egmond.c1v20.-380.mat & $\mathrm{CC}$ & 7144 \\
\hline 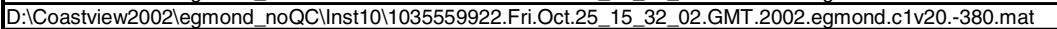 & $\mathrm{CC}$ & 7145 \\
\hline D:ICoastview2002legmond_noQC\Inst10l1035563522.Fri.Oct.25_16_32_02.GMT.2002.egmond.c1v20.-380.mat & $\mathrm{CC}$ & 7146 \\
\hline D:ICoastview2002legmond_noQC Inst1011035613922.Sat.Oct.26_06_32_02.GMT.2002.egmond.c1v20.-380.mat & $\mathrm{CC}$ & 7160 \\
\hline D:ICoastview2002legmond_noQClInst1011035617522.Sat.Oct.26_07_32_02.GMT.2002.egmond.c1v20.-380.mat & $\mathrm{CC}$ & 7161 \\
\hline D:ICoastview2002legmond_noQC|Inst1011035621122.Sat.Oct.26_08_32_02.GMT.2002.egmond.c1v20.-380.mat & $\mathrm{CC}$ & 7162 \\
\hline D:ICoastview2002legmond_noQCl|nst1011035624722.Sat.Oct.26_09_32_02.GMT.2002.egmond.c1v20.-380.mat & $\mathrm{CC}$ & 7163 \\
\hline D:ICoastview2002legmond_noQClInst1011035628321.Sat.Oct.26_10_32_01.GMT.2002.egmond.c1v20.-380.mat & $\mathrm{CC}$ & 7164 \\
\hline D:ICoastview2002legmond_noQC/Inst1011035631922.Sat.Oct.26_11_32_02.GMT.2002.egmond.c1v20.-380.mat & CC & 7165 \\
\hline D:ICoastview2002legmond_noQClInst1011035635524.Sat.Oct.26_12_32_04.GMT.2002.egmond.c1v20.-380.mat & $\mathrm{CC}$ & 7166 \\
\hline D:ICoastview2002legmond_noQClInst1011035639121.Sat.Oct.26_13_32_01.GMT.2002.egmond.c1v20.-380.mat & $\mathrm{CC}$ & 7167 \\
\hline D:ICoastview2002legmond_noQClInst1011035642722.Sat.Oct.26_14_32_02.GMT.2002.egmond.c1v20.-380.mat & $\mathrm{CC}$ & 7168 \\
\hline D:ICoastview2002legmond_noQClInst10l1035646321.Sat.Oct.26_15_32_01.GMT.2002.egmond.c1v20.-380.mat & $\mathrm{CC}$ & 7169 \\
\hline 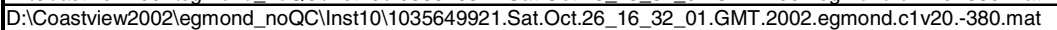 & $\mathrm{CC}$ & 7170 \\
\hline D:ICoastview2002legmond_noQCVInst1011035700322.Sun.0ct.27_06_32_02.GMT.2002.egmond.c1v20.-380.mat & $\mathrm{CC}$ & 7184 \\
\hline D:ICoastview2002legmond_noQCVInst1011035703922.Sun.Oct.27_07_32_02.GMT.2002.egmond.c1v20.-380.mat & $\mathrm{CC}$ & 7185 \\
\hline D:ICoastview2002legmond_noQClInst1011035707522.Sun.Oct.27_08_32_02.GMT.2002.egmond.c1v20.-380.mat & 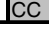 & 7186 \\
\hline
\end{tabular}




\begin{tabular}{|c|c|c|}
\hline D:ICoastview2002legmond_noQC\Inst1011035711122.Sun.Oct.27_09_32_02.GMT.2002.egmond.c1v2C & $\mathrm{CC}$ & 7187 \\
\hline D:ICoastview2002legmond_noQC\Inst1011035714721.Sun.Oct.27_10_32_01.GMT.2002.egmond.c1v20.-380.mat & CC & 7188 \\
\hline D:ICoastview2002legmond_noQClInst1011035718322.Sun.Oct.27 11 32_02.GMT.2002.egmond.c1v20.-380.mat & $\mathrm{CC}$ & 7189 \\
\hline D:ICoastview2002legmond_noQCVInst1011035721922.Sun.Oct.27_12_32_02.GMT.2002.egmond.c1v20.-380.mat & $\mathrm{CC}$ & 7190 \\
\hline D:ICoastview2002legmond_noQC\Inst1011035725522.Sun.Oct.27_13_32_02.GMT.2002.egmond.c1v20.-380.mat & $\mathrm{CC}$ & 7191 \\
\hline D:ICoastview2002legmond_noQCVInst1011035729122.Sun.Oct.27_14_32_02.GMT.2002.egmond.c1v20.-380.mat & $\mathrm{CC}$ & 7192 \\
\hline D:ICoastview2002legmond_noQC\Inst101035732721.Sun.Oct.27_15_32_01.GMT.2002.egmond.c1v20.-380.mat & $\mathrm{CC}$ & 7193 \\
\hline D:ICoastview2002legmond_noQCVInst1011035736322.Sun.Oct.27_16_32_02.GMT.2002.egmond.c1v20.-380.mat & $\mathrm{CC}$ & 7194 \\
\hline D:ICoastview2002legmond_noQC|Inst1011035786722.Mon.Oct.28_06_32_02.GMT.2002.egmond.c1v20.-380.mat & CA & 7208 \\
\hline D:ICoastview2002legmond_noQClInst1011035790321.Mon.Oct.28_07_32_01.GMT.2002.egmond.c1v20.-380.mat & CA & 7209 \\
\hline D:ICoastview2002legmond_noQC|Inst1011035793921.Mon.Oct.28_08_32_01.GMT.2002.egmond.c1v20.-380.mat & CA & 7210 \\
\hline D:ICoastview2002legmond_noQClInst1011035797522.Mon.Oct.28_09_32_02.GMT.2002.egmond.c1v20.-380.mat & CA & 7211 \\
\hline D:ICoastview2002legmond_noQCVInst10l1035801122.Mon.Oct.28_10_32_02.GMT.2002.egmond.c1v20.-380.mat & $\mathrm{CA}$ & 7212 \\
\hline D:ICoastview2002legmond_noQClInst1011035804722.Mon.Oct.28_11_32_02.GMT.2002.egmond.c1v20.-380.mat & CA & 7213 \\
\hline D:ICoastview2002legmond_noQClInst1011035808321.Mon.Oct.28_12_32_01.GMT.2002.egmond.c1v20.-380.mat & $\mathrm{CA}$ & 7214 \\
\hline D:ICoastview2002legmond_noQCVInst1011035811922.Mon.Oct.28_13 32_02.GMT.2002.egmond.c1v20.-380.mat & $\mathrm{CA}$ & 7215 \\
\hline D:ICoastview2002legmond_noQC|Inst1011035815522.Mon.Oct.28_14_32_02.GMT.2002.egmond.c1v20.-380.mat & $\mathrm{CA}$ & 7216 \\
\hline D:ICoastview2002legmond_noQC|Inst10l1035819121.Mon.Oct.28_15_32_01.GMT.2002.egmond.c1v20.-380.mat & $\mathrm{CA}$ & 7217 \\
\hline D:ICoastview2002legmond_noQC|Inst1011035822722.Mon.Oct.28_16_32_02.GMT.2002.egmond.c1v20.-380.mat & $\mathrm{CA}$ & 7218 \\
\hline D:ICoastview2002legmond_noQCVInst1011035873122.Tue.Oct.29_06_32_02.GMT.2002.egmond.c1v20.-380.mat & CB & 7232 \\
\hline D:ICoastview2002legmond_noQC\Inst1011035876722.Tue.Oct.29 07 32_02.GMT.2002.egmond.c1v20.-380.mat & $\mathrm{CB}$ & 7233 \\
\hline D:ICoastview2002legmond_noQC\Inst101035880322.Tue.Oct.29_08_32_02.GMT.2002.egmond.c1v20.-380.mat & CB & 7234 \\
\hline D:ICoastview2002legmond_noQCVInst1011035883921.Tue.Oct.29_09_32_01.GMT.2002.egmond.c1v20.-380.mat & CB & 7235 \\
\hline D:ICoastview2002legmond_noQCVInst1011035887522.Tue.Oct.29_10_32_02.GMT.2002.egmond.c1v20.-380.mat & CB & 7236 \\
\hline D:ICoastview2002legmond_noQCVInst1011035891122.Tue.Oct.29_11_32_02.GMT.2002.egmond.c1v20.-380.mat & $\mathrm{CB}$ & 7237 \\
\hline D:ICoastview2002legmond_noQCVInst1011035894721.Tue.Oct.29_12_32_01.GMT.2002.egmond.c1v20.-380.mat & CB & 7238 \\
\hline D:ICoastview2002legmond_noQClInst1011035898321.Tue.Oct.29_13_32_01.GMT.2002.egmond.c1v20.-380.mat & $\mathrm{CB}$ & 7239 \\
\hline D:ICoastview2002legmond_noQC\Inst101035901922.Tue.Oct.29_14_32_02.GMT.2002.egmond.c1v20.-380.mat & CB & 7240 \\
\hline D:ICoastview2002legmond_noQC\Inst101035905522.Tue.Oct.29_15_32_02.GMT.2002.egmond.c1v20.-380.mat & $\mathrm{CB}$ & 7241 \\
\hline D:ICoastview2002legmond_noQC\Inst1011035909122.Tue.Oct.29_16_32_02.GMT.2002.egmond.c1v20.-380.mat & $\mathrm{CB}$ & 7242 \\
\hline D:ICoastview2002legmond_noQC|Inst1011035959522.Wed.Oct.30_06_32_02.GMT.2002.egmond.c1v20.-380.mat & CB & 7256 \\
\hline D:ICoastview2002legmond_noQC|Inst1011035963121.Wed.Oct.30_07_32_01.GMT.2002.egmond.c1v20.-380.mat & CB & 7257 \\
\hline D:ICoastview2002legmond_noQC|Inst1011035966721.Wed.Oct.30_08_32_01.GMT.2002.egmond.c1v20.-380.mat & $\mathrm{CB}$ & 7258 \\
\hline D:ICoastview2002legmond_noQCl|nst1011035970323.Wed.Oct.30_09_32_03.GMT.2002.egmond.c1v20.-380.mat & CB & 7259 \\
\hline D:ICoastview2002legmond_noQClInst1011035973921.Wed.Oct.30_10_32_01.GMT.2002.egmond.c1v20.-380.mat & CB & 7260 \\
\hline D:ICoastview2002legmond_noQCVInst10l1035977521.Wed.Oct.30_11_32_01.GMT.2002.egmond.c1v20.-380.mat & $\mathrm{CB}$ & 7261 \\
\hline D:ICoastview2002legmond_noQC|Inst1011035981121.Wed.Oct.30_12_32_01.GMT.2002.egmond.c1v20.-380.mat & $\mathrm{CB}$ & 7262 \\
\hline D:ICoastview2002legmond_noQC|Inst1011035984721.Wed.Oct.30_13_32_01.GMT.2002.egmond.c1v20.-380.mat & CB & 7263 \\
\hline D:ICoastview2002legmond_noQC|Inst1011035988321.Wed.Oct.30_14_32_01.GMT.2002.egmond.c1v20.-380.mat & $\mathrm{CB}$ & 7264 \\
\hline D:ICoastview2002legmond_noQC|Inst1011035991921.Wed.Oct.30_15_32_01.GMT.2002.egmond.c1v20.-380.mat & $\mathrm{CB}$ & 7265 \\
\hline D:ICoastview2002legmond_noQC|Inst1011035995522.Wed.Oct.30_16_32_02.GMT.2002.egmond.c1v20.-380.mat & CB & 7266 \\
\hline D:ICoastview2002legmond_noQC|Inst1011036045922.Thu.Oct.31_06_32_02.GMT.2002.egmond.c1v20.-380.mat & CB & 7280 \\
\hline D:ICoastview2002legmond_noQCVInst1011036049522.Thu.Oct.31_07_32_02.GMT.2002.egmond.c1v20.-380.mat & CB & 7281 \\
\hline D:ICoastview2002legmond_noQCVInst101036053122.Thu.Oct.31_08_32_02.GMT.2002.egmond.c1v20.-380.mat & CB & 7282 \\
\hline D:ICoastview2002legmond_noQCVInst101036056722.Thu.Oct.31_09_32_02.GMT.2002.egmond.c1v20.-380.mat & CB & 7283 \\
\hline D:ICoastview2002legmond_noQCVInst101036060321.Thu.Oct.31_10_32_01.GMT.2002.egmond.c1v20.-380.mat & CB & 7284 \\
\hline D:ICoastview2002legmond_noQCVInst1011036063921.Thu.Oct.31_11_32_01.GMT.2002.egmond.c1v20.-380.mat & CB & 7285 \\
\hline D:ICoastview2002legmond_noQC\Inst1011036067522.Thu.Oct.31_12_32_02.GMT.2002.egmond.c1v20.-380.mat & CB & 7286 \\
\hline D:ICoastview2002legmond_noQC\Inst101036071122.Thu.Oct.31_13_32_02.GMT.2002.egmond.c1v20.-380.mat & CB & 7287 \\
\hline D:ICoastview2002legmond_noQC Inst1011036074721.Thu.Oct.31_14_32_01.GMT.2002.egmond.c1v20.-380.mat & CB & 7288 \\
\hline D:ICoastview2002legmond_noQC \Inst1011036078322.Thu.Oct.31_15_32_02.GMT.2002.egmond.c1v20.-380.mat & CB & 7289 \\
\hline D:ICoastview2002legmond_noQCIInst1011036081922.Thu.Oct.31_16_32_02.GMT.2002.egmond.c1v20.-380.mat & СB & 7290 \\
\hline \multicolumn{3}{|l|}{ Instrument 13 } \\
\hline D:ICoastview2002legmond_noQCVInst13\1035354721.Wed.Oct.23_06_32_01.GMT.2002.E & $\mathrm{CA}$ & 7088 \\
\hline D:ICoastview2002legmond_noQC|Inst13\1035358322.Wed.Oct.23_07_32_02.GMT.2002.egmond.c1v30.-380.mat & CA & 7089 \\
\hline D:ICoastview2002legmond_noQC|Inst13\1035361921.Wed.Oct.23_08_32_01.GMT.2002.egmond.c1v30.-380.mat & CA & 7090 \\
\hline D:ICoastview2002legmond noQCl|nst13\1035365521.Wed.Oct.23 09 32 01.GMT.2002.egmond.c1v30.-380.mat & CA & 7091 \\
\hline D:ICoastview2002legmond_noQC|Inst13\1035369122.Wed.Oct.23_10_32_02.GMT.2002.egmond.c1v30.-380.mat & CA & 7092 \\
\hline D:ICoastview2002legmond_noQC)Inst13\1035372722.Wed.Oct.23_11_32_02.GMT.2002.egmond.c1v30.-380.mat & CA & 7093 \\
\hline D:ICoastview2002legmond_noQC|Inst13\1035376321.Wed.Oct.23_12_32_01.GMT.2002.egmond.c1v30.-380.mat & CA & 7094 \\
\hline D:ICoastview2002legmond_noQCVInst1311035379922.Wed.Oct.23_13_32_02.GMT.2002.egmond.c1v30.-380.mat & CA & 7095 \\
\hline D:ICoastview2002legmond_noQC|Inst13\1035383522.Wed.Oct.23_14_32_02.GMT.2002.egmond.c1v30.-380.mat & CA & 7096 \\
\hline D:ICoastview2002legmond_noQC|Inst13\1035387122.Wed.Oct.23_15_32_02.GMT.2002.egmond.c1v30.-380.mat & CA & 7097 \\
\hline D:ICoastview2002legmond_noQC|Inst13\1035390722.Wed.Oct.23_16_32_02.GMT.2002.egmond.c1v30.-380.mat & CA & 7098 \\
\hline D:ICoastview2002legmond_noQC|Inst13/1035441122.Thu.Oct.24_06_32_02.GMT.2002.egmond.c1v30.-380.mat & CC & 7112 \\
\hline D:ICoastview2002legmond_noQCVInst13\1035444721.Thu.Oct.24_07_32_01.GMT.2002.egmond.c1v30.-380.mat & $\mathrm{CC}$ & 7113 \\
\hline D:ICoastview2002legmond_noQCVInst13|1035448322.Thu.Oct.24_08_32_02.GMT.2002.egmond.c1v30.-380.mat & CC & 7114 \\
\hline D:ICoastview2002legmond_noQCVInst13|1035451921.Thu.Oct.24_09_32_01.GMT.2002.egmond.c1v30.-380.mat & CC & 7115 \\
\hline D:ICoastview2002legmond_noQCVInst13/1035455522.Thu.Oct.24_10_32_02.GMT.2002.egmond.c1v30.-380.mat & CC & 7116 \\
\hline D:ICoastview2002legmond_noQClInst13/1035459122.Thu.Oct.24_11_32_02.GMT.2002.egmond.c1v30.-380.mat & CC & 7117 \\
\hline D:ICoastview2002legmond_noQC Inst13|1035462722.Thu.Oct.24_12_32_02.GMT.2002.egmond.c1v30.-380.mat & CC & 7118 \\
\hline D:ICoastview2002legmond_noQClInst13|1035466321.Thu.Oct.24_13_32_01.GMT.2002.egmond.c1v30.-380.mat & CC & 7119 \\
\hline D:ICoastview2002legmond_noQCVInst13/1035469922.Thu.Oct.24_14_32_02.GMT.2002.egmond.c1v30.-380.mat & CC & 7120 \\
\hline D:ICoastview2002legmond_noQClInst13\1035473522.Thu.Oct.24_15_32_02.GMT.2002.egmond.c1v30.-380.mat & ICC & 7121 \\
\hline
\end{tabular}


D:ICoastview2002legmond_noQC\Inst13\1035477122.Thu.Oct.24_16_32_02.GMT.2002.egmond.c1v30.-380.mat D:ICoastview2002legmond_noQCVInst13l1035527522.Fri.Oct.25_06_32_02.GMT.2002.egmond.c1v30.-380.mat D:ICoastview2002legmond_noQCVInst13\1035531121.Fri.Oct.25_07_32_01.GMT.2002.egmond.c1v30.-380.mat D:ICoastview2002legmond_noQC \Inst13\1035534721.Fri.Oct.25_08_32_01.GMT.2002.egmond.c1v30.-380.mat D:ICoastview2002legmond_noQClInst13\1035538321.Fri.Oct.25_09_32_01.GMT.2002.egmond.c1v30.-380.mat D:ICoastview2002legmond_noQClInst13|1035541921.Fri.Oct.25_10_32_01.GMT.2002.egmond.c1v30.-380.mat D:ICoastview2002legmond_noQCVInst13\1035545521.Fri.Oct.25_11_32_01.GMT.2002.egmond.c1v30.-380.mat D:ICoastview2002legmond_noQClInst13|1035549122.Fri.Oct.25_12_32_02.GMT.2002.egmond.c1v30.-380.mat D:ICoastview2002legmond_noQCVInst13\1035552722.Fri.Oct.25_13_32_02.GMT.2002.egmond.c1v30.-380.mat D:ICoastview2002legmond_noQCVInst13\1035556322.Fri.Oct.25_14_32_02.GMT.2002.egmond.c1v30.-380.mat D:ICoastview2002legmond_noQCVInst13l1035559922.Fri.Oct.25_15_32_02.GMT.2002.egmond.c1v30.-380.mat D:ICoastview2002legmond_noQCVInst13/1035563522.Fri.Oct.25_16_32_02.GMT.2002.egmond.c1v30.-380.mat D:ICoastview2002legmond_noQC $\mid$ nnst1311035613922.Sat.Oct.26_06_32_02.GMT.2002.egmond.c1v30.-380.mat D:ICoastview2002legmond noQClInst13/1035617522. Sat.Oct.26 0732 02.GMT.2002. egmond.c1v30-380 mat D.ICoastview2002legmond noOCVnst1311035621122 Sat.Oct 26 08 32 02 GMT 2002 egmond.c1v30 -380.mat

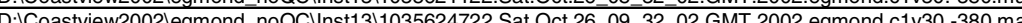

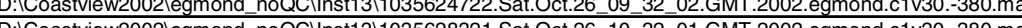
D.ICoasvien2002 D:ICoastview2002legmond_noQCVInst1311035631922.Sat.Oct.26_11_32_02.GMT.2002.egmond.c1v30.-380.mat D:ICoastview2002legmond_noQCVInst13\1035635524.Sat.Oct.26_12_32_04.GMT.2002.egmond.c1v30.-380.mat D:ICoastview2002legmond_noQClInst13/1035639121.Sat.Oct.26_13_32_01.GMT.2002.egmond.c1v30.-380.mat D:ICoastview2002legmond noQCVInst13\1035642722.Sat.Oct.26 14 32 $02 . G M T .2002$.egmond.c1v30.-380.mat D:ICoastview2002legmond noQCVInst13l1035646321. Sat.Oct.26 15 32 01.GMT.2002.egmond.c1v30 -380 ma D.ICoastview2002legmond noQCVInst1311035649921. Sat.Oct.26 16 32 01.GMT 2002 egmond.c1v30.-380.mat D:ICoastview2002legmond_noQCVInst13\1035700322.Sun.Oct.27_06_32_02.GMT.2002.egmond.c1v30.-380.mat D:ICoastview2002legmond_noQClInst13\1035703922.Sun.Oct.27_07_32_02.GMT.2002.egmond.c1v30.-380.mat D:ICoastview2002legmond_noQClInst1311035707522.Sun.Oct.27_08_32_02.GMT.2002.egmond.c1v30.-380.mat D:ICoastview2002legmond_noQCVInst1311035711122.Sun.Oct.27_09_32_02.GMT.2002.egmond.c1v30.-380.mat D:ICoastview2002legmond_noQClInst13\1035714721.Sun.Oct.27_10_32_01.GMT.2002.egmond.c1v30.-380.mat D:ICoastview2002legmond_noQCVInst1311035718322.Sun.Oct.27_11_32_02.GMT.2002.egmond.c1v30.-380.mat D:ICoastview2002legmond_noQCVInst1311035721922.Sun.Oct.27_12_32_02.GMT.2002.egmond.c1v30.-380.mat D:ICoastview2002legmond_noQCVInst13\1035725522.Sun.Oct.27_13_32_02.GMT.2002.egmond.c1v30.-380.mat D:ICoastview2002legmond noQCVInst13\1035729122.Sun.Oct.27_14_32_02.GMT.2002.egmond.c1v30.-380.mat D:ICoastview2002legmond_noQClInst13|1035732721.Sun.Oct.27_15_32_01.GMT.2002.egmond.c1v30.-380.mat D:ICoastview2002legmond noQCVInst1311035736322.Sun.0ct27_16 32 02.GMT 2002 egmond.c1v30.380.mat D:ICoastview2002legmond_noQClInst1311035786722.Mon.Oct.28_06_32_02.GMT.2002.egmond.c1v30.-380.mat D:ICoastview2002legmond_noQClInst13\1035790321.Mon.Oct.28_07_32_01.GMT.2002.egmond.c1v30.-380.mat D:ICoastview2002legmond_noQCVInst1311035793921.Mon.Oct.28_08_32_01.GMT.2002.egmond.c1v30.-380.mat D:ICoastview2002legmond noQCVInst1311035797522.Mon.Oct.28 09 32 02.GMT.2002.egmond.c1v30.-380.mat D:ICoastview2002legmond noQCVInst13/1035801122.Mon.Oct.28 10 32 02.GMT.2002.egmond.c1v30.-380.mat D:ICoastview2002legmond noQCVInst13\1035804722.Mon.Oct.28 11 32 02.GMT.2002.egmond.c1v30.-380.mat D:ICoastview2002legmond_noQClInst13\1035808321.Mon.Oct.28_12_32_01.GMT.2002.egmond.c1v30.-380.mat D:ICoastview2002legmond_noQC|Inst13\1035811922.Mon.Oct.28_13_32_02.GMT.2002.egmond.c1v30.-380.mat D:ICoastview2002legmond_noQCVInst13\1035815522.Mon.Oct.28_14_32_02.GMT.2002.egmond.c1v30.-380.mat D:ICoastview2002legmond_noQClInst13 11035819121.Mon.Oct.28_15_32_01.GMT.2002.egmond.c1v30.-380.mat D:ICoastview2002legmond_noQC \Inst13\1035822722.Mon.Oct.28_16_32_02.GMT.2002.egmond.c1v30.-380.mat D:ICoastview2002legmond_noQCVInst13\1035873122.Tue.Oct.29_06_32_02.GMT.2002.egmond.c1v30.-380.mat D:ICoastview2002legmond_noQClInst13\1035876722.Tue.Oct.29_07_32_02.GMT.2002.egmond.c1v30.-380.mat D:ICoastview2002legmond_noQClInst13\1035880322.Tue.Oct.29_08_32_02.GMT.2002.egmond.c1v30.-380.mat D:ICoastview2002legmond_noQC Inst1311035883921.Tue.Oct.29_09_32_01.GMT.2002.egmond.c1v30.-380.ma D:ICoastview2002legmond_noQClInst13\1035887522.Tue.Oct.29_10_32_02.GMT.2002.egmond.c1v30.-380.mat D:ICoastview2002legmond_noQClInst13\1035891122.Tue.Oct.29_11_32_02.GMT.2002.egmond.c1v30.-380.mat D:ICoastview2002legmond_noQCVInst13\1035894721.Tue.Oct.29_12_32_01.GMT.2002.egmond.c1v30.-380.mat D:ICoastview2002legmond_noQCVInst13\1035898321.Tue.Oct.29_13_32_01.GMT.2002.egmond.c1v30.-380.mat D:ICoastview2002legmond noQCl|nst13/1035901922.Tue.Oct.29 14 32 02.GMT.2002.egmond.c1v30.-380.mat DilCoastview2002legmond noQClnst1311035905522.Tue.Oct.29 15 32 02 .GMT.2002.egmond.c1v30.-380.mat D:ICoastview2002legmond_noQCVInst1311035905522.Tue.Oct.29_15_32_02.GMT.2002.egmond.c1v30.-380.mat D:ICoastview2002legmond_noQC|Inst13/1035959522.Wed.Oct.30_06_32_02.GMT.2002.egmond.c1v30.-380.mat D:ICoastview2002legmond_noQClInst13\1035963121.Wed.Oct.30_07_32_01.GMT.2002.egmond.c1v30.-380.mat D:ICoastview2002legmond_noQCVInst13|1035966721.Wed.Oct.30_08_32_01.GMT.2002.egmond.c1v30.-380.mat D:ICoastview2002legmond_noQCVInst1311035970323.Wed.Oct.30_09_32_03.GMT.2002.egmond.c1v30.-380.mat D:ICoastview2002legmond_noQClInst13\1035973921.Wed.Oct.30_10_32_01.GMT.2002.egmond.c1v30.-380.mat D:ICoastview2002legmond noQCVInst13\1035977521.Wed.Oct.30 1132 01.GMT.2002.egmond.c1v30.-380.mat D:ICoastview2002legmond noQClInst13/1035981121.Wed.Oct.30 1232 01.GMT.2002.egmond.c1v30.-380.mat D:ICoastview2002legmond_noQCVInst13\1035984721.Wed.Oct.30_13_32_01.GMT.2002.egmond.c1v30.-380.mat D:ICoastview2002legmond_noQCVInst13\1035988321.Wed.Oct.30_14_32_01.GMT.2002.egmond.c1v30.-380.mat D:ICoastview2002legmond_noQClInst13\1035991921.Wed.Oct.30_15_32_01.GMT.2002.egmond.c1v30.-380.mat D:ICoastview2002legmond_noQCVInst13\1035995522.Wed.Oct.30_16_32_02.GMT.2002.egmond.c1v30.-380.mat D:ICoastview2002legmond_noQClInst13\1036045922.Thu.Oct.31_06_32_02.GMT.2002.egmond.c1v30.-380.mat D:ICoastview2002legmond noQCVInst13\1036049522.Thu.Oct.31_07_32_02.GMT.2002.egmond.c1v30.-380.mat D:ICoastview2002legmond noQCVInst13\1036053122.Thu.Oct.31_08_32_02.GMT.2002.egmond.c1v30.-380.mat D:ICoastview2002legmond noQCVInst13\1036056722. Thu.Oct.31 09 32 02.GMT.2002.egmond.c1v30.-380.mat D:ICoastview2002legmond_noQC/|nst1311036056722. Thu.Oct.31_09_32_02.GMT.2002.egmond.c1v30.-380.mat D:ICoastview2002legmond_noQC \Inst13\1036060321. Thu.Oct.31_10_32_01.GMT.2002.egmond.c1v30.-380.mat D:ICoastview2002legmond_noQCVInst13/1036067522.Thu.Oct.31_12_32_02.GMT.2002.egmond.c1v30.-380.mat D:ICoastview2002legmond_noQCVInst13\1036071122.Thu.Oct.31_13_32_02.GMT.2002.egmond.c1v30.-380.mat D:ICoastview2002legmond noOClnst13\1036074721.Thu Oct.31 1432 01.GMT 2002.egmond.c1v30-380.ma D:ICoastview2002legmond noQCVInst1311036078322.Thu.Oct.31 15 32 02.GMT.2002.egmond.c1v30.-380.ma D:ICoastview2002legmond_noQCVInst13\1036081922.Thu.Oct.31_16_32_02.GMT.2002.egmond.c1v30.-380.mat

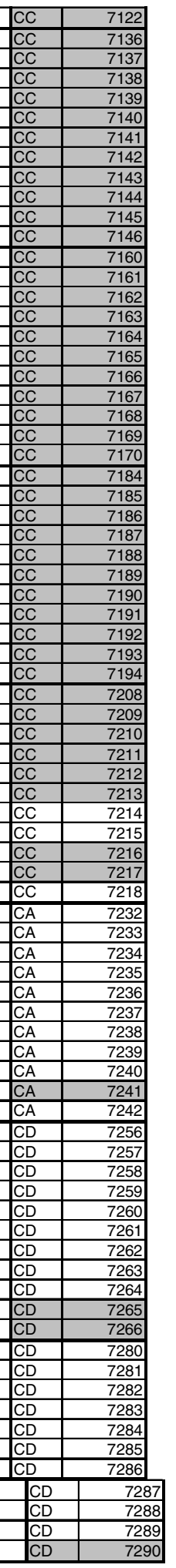




\section{Noordwijk (video and ground truth file, gray=not useful)}

\begin{tabular}{|c|c|c|}
\hline \multicolumn{3}{|l|}{ Instrument10 } \\
\hline D:Woordwijk 2003|nordzee1_noQCVInst1011049729435.Mon.Apr.07_15_30_35.GMT.2003.nordzee1.v150.170.mat & & 2320 \\
\hline D:Woordwijk 2003|nordzee1_noQCVInst10|1049733034.Mon.Apr.07_16_30_34.GMT.2003.nordzee1.v150.170.mat & & 2321 \\
\hline 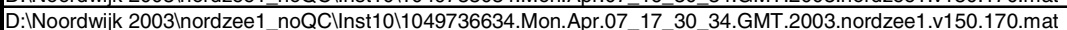 & & \\
\hline 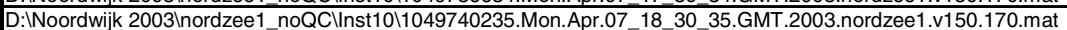 & & 2323 \\
\hline D:Woordwijk 2003ไnordzee1_noQCVInst1011049743834.Mon.Apr.07_19_30_34.GMT.2003.nordzee1.v150.170.mat & & 2324 \\
\hline D:Woordwijk 2003|nordzee1_noQCVInst1011049765437.Tue.Apr.08_01_30_37.GMT.2003.nordzee1.v150.170.mat & & 2330 \\
\hline 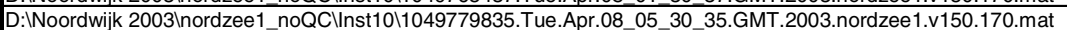 & & 2334 \\
\hline 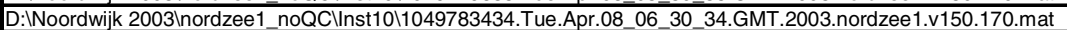 & & 2335 \\
\hline D:Woordwijk 2003lnordzee1 noQCVInst10\1049787035.Tue.Apr.08 07 30 35.GMT.2003.nordzee1.v150.170.mat & & 2336 \\
\hline D:INoordwijk 2003/nordzee1_noQC/Inst10l1049790634.Tue.Apr.08_08_30_34.GMT.2003.nordzee1.v150.170.mat & & 2337 \\
\hline D:Woordwijk 2003lnordzee1_noQClInst10l1049794235.Tue.Apr.08 09 30 35.GMT.2003.nordzee1.v150.170.mat & & \\
\hline D:Woordwijk 2003lnordzee1_noQCl|nst10l1049797835.Tue.Apr.08_10_30_35.GMT.2003.nordzee1.v150.170.mat & & 2339 \\
\hline D:Woordwijk 2003ไnordzee1_noQClInst1011049801435.Tue.Apr.08_11_30_35.GMT.2003.nordzee1.v150.170.mat & & 2340 \\
\hline D:Woordwijk 2003lnordzee1 noQCl|nst10l1049805035.Tue.Apr.08 12 30 35. GMT.2003.nordzee1.v150.170.mat & & 2341 \\
\hline 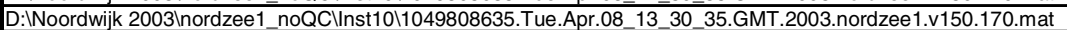 & & 2342 \\
\hline D:Woordwijk 2003lnordzee1_noQCl|nst10\1049812235.Tue.Apr.08 14 30 35.GMT.2003.nordzee1.v150.170.mat & & 2343 \\
\hline 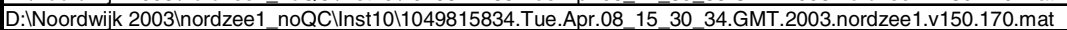 & & 2344 \\
\hline D:INoordwijk 2003|nordzee1_noQCl|nst10l1049819434.Tue.Apr.08_16_30_34.GMT.2003.nordzee1.v150.170.mat & & 2345 \\
\hline D:Woordwijk 2003ไnordzee1_noQCVInst1011049823035.Tue.Apr.08_17_30_35.GMT.2003.nordzee1.v150.170.mat & & 2346 \\
\hline D:Noordwijk 2003|nordzee1_noQC\Inst10\1049826635.Tue.Apr.08_18_30_35.GMT.2003.nordzee1.v150.170.mat & & 2347 \\
\hline D:INoordwijk 2003lnordzee1_noQC|Inst10l1049830234.Tue.Apr.08_19 30_34.GMT.2003.nordzee1.v150.170.mat & & 2348 \\
\hline D:WNoordwijk 2003lnordzee1_noQCVInst10\1049851837.Wed.Apr.09_01_30_37.GMT.2003.nordzee1.v150.170.mat & & 2354 \\
\hline D:INoordwijk 2003lnordzee1_noQCVInst10|1049866236.Wed.Apr.09_05_30_36.GMT.2003.nordzee1.v150.170.mat & & 2358 \\
\hline 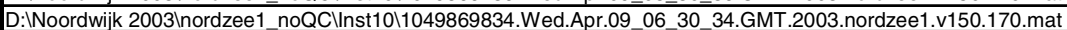 & & 2359 \\
\hline 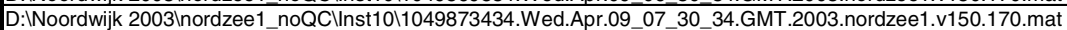 & & 2360 \\
\hline D:INoordwijk 2003lnordzee1_noQCVInst1011049877034.Wed.Apr.09_08_30_34.GMT.2003.nordzee1.v150.170.mat & & 2361 \\
\hline D:Woordwijk 2003/nordzee1_noQCVInst1011049880632.Wed.Apr.09_09_30_32.GMT.2003.nordzee1.v150.170.mat & & 2362 \\
\hline D:Woordwijk 2003lnordzee1_noQCVInst1011049884235.Wed.Apr.09_10_30_35.GMT.2003.nordzee1.v150.170.mat & & 2363 \\
\hline D:INoordwijk 2003lnordzee1_noQCVInst1011049887835.Wed.Apr.09_11_30_35.GMT.2003.nordzee1.v150.170.mat & & 2364 \\
\hline D:Woordwijk 2003lnordzee1_noQCVInst1011049891434.Wed.Apr.09_12_30_34.GMT.2003.nordzee1.v150.170.mat & & 2365 \\
\hline D:Woordwijk 2003|nordzee1_noQCVInst1011049895035.Wed.Apr.09_13_30_35.GMT.2003.nordzee1.v150.170.mat & & 2366 \\
\hline D:INoordwijk 2003lnordzee1_noQCVInst10\1049898634.Wed.Apr.09_14_30_34.GMT.2003.nordzee1.v150.170.mat & & 2367 \\
\hline D:INoordwijk 2003lnordzee1_noQCVInst10\1049902234.Wed.Apr.09_15_30_34.GMT.2003.nordzee1.v150.170.mat & & 2368 \\
\hline D:Woordwijk 2003lnordzee1 noQCVInst10\1049905834.Wed.Apr.09 16 30 34. GMT.2003.nordzee1.v150.170.mat & & 2369 \\
\hline D:Noordwijk 2003ไnordzee1_noQCVInst10\1049909434.Wed.Apr.09_17_30_34.GMT.2003.nordzee1.v150.170.mat & & 2370 \\
\hline D:INoordwijk 2003lnordzee1_noQCVInst10\1049913035.Wed.Apr.09_18 30 35. GMT.2003.nordzee1.v150.170.mat & & 2371 \\
\hline D:Woordwijk 2003lnordzee1_noQCVInst1011049916634.Wed.Apr.09_19_30_34.GMT.2003.nordzee1.v150.170.mat & & 2372 \\
\hline D:Woordwijk 2003!nordzee1_noQCVInst1011049938236.Thu.Apr.10_01_30_36.GMT.2003.nordzee1.v150.170.mat & & 2378 \\
\hline D:Woordwijk 2003lnordzee1 noQCVInst10\1049952636.Thu.Apr.10 05 30 36.GMT.2003.nordzee1.v150.170.mat & & 2382 \\
\hline 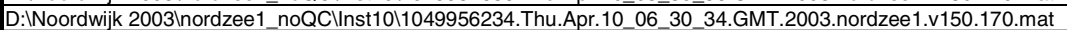 & & 2383 \\
\hline D:INoordwijk 2003|nordzee1_noQC\Inst10\1049959835.Thu.Apr.10_07_30_35.GMT.2003.nordzee1.v150.170.mat & & 2384 \\
\hline D:Woordwijk 2003ไnordzee1_noQClInst10l1049963435.Thu.Apr.10_08_30_35.GMT.2003.nordzee1.v150.170.mat & & 2385 \\
\hline D:Woordwijk 2003lnordzee1_noQCVInst1011049967034.Thu.Apr.10_09_30_34.GMT.2003.nordzee1.v150.170.mat & & 2386 \\
\hline D:Noordwijk 2003|nordzee1_noQCVInst1011049970635.Thu.Apr.10_10_30_35.GMT.2003.nordzee1.v150.170.mat & & 2387 \\
\hline D:Woordwijk 2003lnordzee1_noQCVInst10l1049974231.Thu.Apr.10 11 30 31.GMT.2003.nordzee1.v150.170.mat & & 2388 \\
\hline D:Woordwijk 2003lnordzee1_noQCVInst10\1049977835.Thu.Apr.10 12 30 35.GMT.2003.nordzee1.v150.170.mat & & 2389 \\
\hline D:Noordwijk 2003lnordzee1_noQCVInst10l1049981435.Thu.Apr.10_13_30_35.GMT.2003.nordzee1.v150.170.mat & & 2390 \\
\hline D:Woordwijk 2003|nordzee1_noQCVInst1011049985034.Thu.Apr.10_14_30_34.GMT.2003.nordzee1.v150.170.mat & & 2391 \\
\hline D:Noordwijk 2003ไnordzee1_noQCVInst10|1049988635.Thu.Apr.10_15_30_35.GMT.2003.nordz & CB & 2392 \\
\hline D:Woordwijk 2003ไnordzee1_noQCVnst1011049992234.Thu.Apr.10_16_30_34.GMT.2003.nordzee1.v150.170.mat & $\mathrm{CA} / \mathrm{CB}$ & 2393 \\
\hline D:Woordwijk 2003\nordzee1_noQCVInst1011049995835.Thu.Apr.10_17_30_35.GMT.2003.nordzee1.v150.170.mat & $\mathrm{CB}$ & 2394 \\
\hline 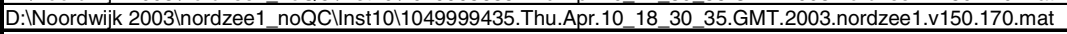 & $\mathrm{CA} / \mathrm{CB}$ & 2395 \\
\hline D:Woordwijk 2003/nordzee1_noQCVInst10\1050003034.Thu.Apr.10_19_30_34.GMT.2003.nordzee1.v150.170.mat & $\mathrm{CA} / \mathrm{CB}$ & 2396 \\
\hline D:INoordwijk 2003|nordzee1_noQCVInst1011050024636.Fri.Apr.11_01_30_36.GMT.2003.nordzee1.v150.170.mat & $\mathrm{CA} / \mathrm{CB}$ & 2402 \\
\hline D:INoordwijk 2003ไnordzee1_noQCVInst1011050039036.Fri.Apr.11_05_30_36.GMT.2003.nordzee1.v150.170.mat & $\mathrm{CA} / \mathrm{CB}$ & 2406 \\
\hline D:INoordwijk 2003/nordzee1_noQCVInst10|1050042635.Fri.Apr.11_06_30_35.GMT.2003.nordzee1.v150.170.mat & $\mathrm{CB}$ & 2407 \\
\hline D:INoordwijk 2003/nordzee1_noQCVInst1011050046234.Fri.Apr.11_07_30_34.GMT.2003.nordzee1.v150.170.mat & $\mathrm{CB}$ & 2408 \\
\hline D:INoordwijk 2003ไnordzee1_noQCVInst1011050049834.Fri.Apr.11_08_30_34.GMT.2003.nordzee1.v150.170.mat & $\mathrm{CB}$ & 2409 \\
\hline D:INoordwijk 2003ไnordzee1_noQCVInst1011050053434.Fri.Apr.11_09_30_34.GMT.2003.nordzee1.v150.170.mat & $\mathrm{CB}$ & 2410 \\
\hline D:INoordwijk 2003ไnordzee1_noQCVInst10|1050057035.Fri.Apr.11_10_30_35.GMT.2003.nordzee1.v150.170.mat & $\mathrm{CB}$ & 2411 \\
\hline D:INoordwijk 2003/nordzee1_noQCVInst10|1050060634.Fri.Apr.11_11_30 34.GMT.2003.nordzee1.v150.170.mat & $\mathrm{CB}$ & 2412 \\
\hline D:INoordwijk 2003/nordzee1_noQCVInst10|1050064231.Fri.Apr.11_12_30_31.GMT.2003.nordzee1.v150.170.mat & $\mathrm{CB}$ & 2413 \\
\hline D:INoordwijk 2003ไnordzee1_noQCVInst10|1050067835.Fri.Apr.11_13_30_35.GMT.2003.nordzee1.v150.170.mat & $\mathrm{CB}$ & 2414 \\
\hline D:INoordwijk 2003lnordzee1_noQCl|nst1011050071435.Fri.Apr.11_14_30_35.GMT.2003.nordzee1.v150.170.mat & $\mathrm{CB}$ & 2415 \\
\hline D:INoordwijk 2003\nordzee1_noQCVInst1011050075034.Fri.Apr.11_15_30_34.GMT.2003.nordzee1.v150.170.mat & $\mathrm{CB}$ & 2416 \\
\hline D:INoordwijk 2003ไnordzee1_noQCVInst10|1050078635.Fri.Apr.11_16_30_35.GMT.2003.nordzee1.v150.170.mat & $\mathrm{CA} / \mathrm{C}$ & 2417 \\
\hline D:INoordwijk 2003ไnordzee1_noQCVInst10|1050082234.Fri.Apr.11_17_30_34.GMT.2003.nordzee1.v150.170.mat & $\mathrm{CA} / \mathrm{C}$ & 2418 \\
\hline D:INoordwijk 2003lnordzee1_noQCVInst1011050085835.Fri.Apr.11_18_30_35.GMT.2003.nordzee1.v150.170.mat & $\mathrm{CA} / \mathrm{CB}$ & 2419 \\
\hline D:INoordwijk 2003ไnordzee1_noQCVInst10|1050089434.Fri.Apr.11_19_30_34.GMT.2003.nordzee1.v150.170.mat & $\mathrm{CA} / \mathrm{CB}$ & 2420 \\
\hline D:INoordwijk 2003ไnordzee1_noQCVInst10l1050111036.Sat.Apr.12_01_30_36.GMT.2003.nordzee1.v150.170.mat & $\mathrm{CA} / \mathrm{CB}$ & 2426 \\
\hline D:Noordwijk 2003ไnordzee1_noQCVInst1011050125436.Sat.Apr.12_05_30_36.GMT.2003.nordzee1.v150.170.mat & $\mathrm{CA} / \mathrm{CB}$ & 2430 \\
\hline D:INoordwijk 2003/nordzee1_noQCVInst1011050129035.Sat.Apr.12_06_30_35.GMT.2003.nordzee1.v150.170.mat & CB & 2431 \\
\hline D:Noordwijk 2003/nordzee1_noQCVInst1011050132634.Sat.Apr.12 07 30 34.GMT.2003.nordzee1.v150.170.mat & CB & \\
\hline
\end{tabular}


D:INoordwijk 2003lnordzee1_noQCIInst1011050136235.Sat.Apr.12_08_30_35.GMT.2003.nordzee1.v150.170.mat D:INoordwijk 2003Inordzee1 noQCVInst1011050139835.Sat.Apr.12 09 30 35.GMT.2003.nordzee1.v150.170.mat D:Noordwijk 2003lnordzee1_noQCVInst1011050143435.Sat.Apr.12_10_30_35.GMT.2003.nordzee1.v150.170.mat D:Noordwijk 2003lnordzee1_noQCVInst10l1050147034.Sat.Apr.12_11_30_34.GMT.2003.nordzee1.v150.170.mat D:Woordwijk 2003lnordzee1_noQCVInst10 1050150634.Sat.Apr.12_12_30_34.GMT.2003.nordzee1.v150.170.mat D:Noordwijk 2003lnordzee1_noQCVInst10\1050154235.Sat.Apr.12_13_30_35.GMT.2003.nordzee1.v150.170.mat D:INoordwijk 2003/nordzee1_noQCVInst10l1050157835.Sat.Apr.12_14_30_35.GMT.2003.nordzee1.v150.170.mat D:Woordwijk 2003ไnordzee1 noQCVInst10l1050161434.Sat.Apr.12 15 30 34.GMT.2003.nordzee1.v150.170.mat D:INoordwijk 2003ไnordzee1_noQCVInst10\1050165034.Sat.Apr.12_16_30_34.GMT.2003.nordzee1.v150.170.mat D:Woordwijk 2003lnordzee1_noQClInst10l1050168634.Sat.Apr.12_17_30_34.GMT.2003.nordzee1.v150.170.mat D:INoordwijk 2003lnordzee1_noQCVInst10l1050172234.Sat.Apr.12_18_30_34.GMT.2003.nordzee1.v150.170.mat D:INoordwijk 2003ไnordzee1_noQCVInst10\1050175835.Sat.Apr.12_19_30_35.GMT.2003.nordzee1.v150.170.mat D:INoordwijk 2003lnordzee1_noQCVInst10l1050197437.Sun.Apr.13_01_30_37.GMT.2003.nordzee1.v150.170.mat D:INoordwijk 2003lnordzee1_noQCVInst1011050211836.Sun.Apr.13_05_30_36.GMT.2003.nordzee1.v150.170.mat D:INoordwijk 2003lnordzee1 noQCVInst1011050215434.Sun.Apr.13 06 30 34.GMT.2003.nordzee1.v150.170.mat D:Woordwijk 2003lnordzee1 noQCVInst1011050219035.Sun.Apr.13_07_30_35.GMT.2003.nordzee1.v150.170.mat D:INoordwijk 2003lnordzee1 noQCVInst10l1050222634.Sun.Apr.13 08 30 34.GMT.2003.nordzee1.v150.170.mat D:Woordwijk 2003lnordzee1_noQCVInst10\1050226235.Sun.Apr.13_09_30_35.GMT.2003.nordzee1.v150.170.mat D:INoordwijk 2003lnordzee1_noQClInst1011050229835.Sun.Apr.13_10_30_35.GMT.2003.nordzee1.v150.170.mat D:Woordwijk 2003lnordzee1_noQCVInst1011050233432.Sun.Apr.13_11_30_32.GMT.2003.nordzee1.v150.170.mat D:Woordwijk 2003lnordzee1 noQCVInst10l1050237034.Sun.Apr.13 12 30 34.GMT.2003.nordzee1.v150.170.mat D:INoordwijk 2003/nordzee1_noQCVInst10l1050240634.Sun.Apr.13_13_30_34.GMT.2003.nordzee1.v150.170.mat D:INoordwijk 2003ไnordzee1 noQCVInst10 1050244234.Sun.Apr.13 14 30 34.GMT.2003.nordzee1.v150.170.mat D:INoordwijk 2003lnordzee1 noQCVInst10l1050247834.Sun.Apr.13_15 30_34.GMT.2003.nordzee1.v150.170.mat D:INoordwijk 2003lnordzee1 noQClInst1011050251435.Sun.Apr.13 $16 \quad 30$ 35.GMT.2003.nordzee1.v150.170.mat D:Woordwijk 2003lnordzee1_noQClnst10l1050255035.Sun.Apr.13 17 30_35.GMT.2003.nordzee1.v150.170.mat D:Woordwijk 2003lnordzee1_noQCVInst1011050258634.Sun.Apr.13_18_30_34.GMT.2003.nordzee1.v150.170.mat D:INoordwijk 2003\nordzee1_noQCVInst10\1050262234.Sun.Apr.13_19_30_34.GMT.2003.nordzee1.v150.170.mat D:Woordwijk 2003lnordzee1_noQCVInst10l1050283837.Mon.Apr.14_01_30_37.GMT.2003.nordzee1.v150.170.mat D:INoordwijk 2003/nordzee1 noQCVnst1011050298236.Mon.Apr.14 05 30 36.GMT.2003.nordzee1.v150.170.mat D:Noordwijk 2003lnordzee1 noQCVInst10ไ1050301835.Mon.Apr.14 06 30 35.GMT.2003.nordzee1.v150.170.mat D.Nordwik 2003 hnordzee1 no0CInst10 1050305434.Mon.Apr.14 0730 34.GMT.2003.nordzee1.v150.170.ma D:INoordwijk 2003/nordzee1_noQCVInst10l1050305434.Mon.Apr.14_07_30_34.GMT.2003.nordzee1.v150.170.mat D:INoordwijk 2003ไnordzee1_noQCVInst10|1050309034.Mon.Apr.14_08_30_34.GMT.2003.nordzee1.v150.170.mat D:INoordwijk 2003lnordzee1 noQCVInst1011050316232.Mon.Apr.14 10 30 32.GMT.2003.nordzee1.v150.170.ma D:Noordwijk 2003lnordzee1_noQCl|nst10l1050319835.Mon.Apr.14_11_30_35.GMT.2003.nordzee1.v150.170.mat D:INoordwijk 2003/nordzee1_noQCVInst10l1050323434.Mon.Apr.14_12_30_34.GMT.2003.nordzee1.v150.170.mat D:INoordwijk 2003lnordzee1 noQCVnst10l1050327035.Mon.Apr.14 13 30 35.GMT.2003.nordzee1.v150.170.mat D:Noordwijk 2003lnordzee1 noQCVInst10 1050330634.Mon.Apr.14 14 30 34.GMT.2003.nordzee1.v150.170.mat D:Woordwijk 2003lnordzee1_noQCVInst10l1050334234.Mon.Apr.14_15_30_34.GMT.2003.nordzee1.v150.170.mat D:INoordwijk 2003lnordzee1_noQCVInst10|1050337834.Mon.Apr.14_16_30_34.GMT.2003.nordzee1.v150.170.ma D:Noordwijk 2003|nordzee1_noQCVInst10l1050341434.Mon.Apr.14_17_30_34.GMT.2003.nordzee1.v150.170.mat D:INoordwijk 2003/nordzee1_noQCVInst10l1050345034.Mon.Apr.14 18 30 34.GMT.2003.nordzee1.v150.170.mat D:Woordwijk 2003lnordzee1_noQCVInst10l1050348634.Mon.Apr.14_19_30_34.GMT.2003.nordzee1.v150.170.mat D:Noordwijk 2003lnordzee1_noQCVInst10l1050370237.Tue.Apr.15_01_30_37.GMT.2003.nordzee1.v150.170.mat D:Woordwijk 2003lnordzee1 noQCVnst1011050384636.Tue.Apr.15 $05 \quad 30$ 36.GMT.2003.nordzee1.v150.170.mat D:Woordwijk 2003lnordzee1_noQCVInst10\1050388234.Tue.Apr.15_06_30_34.GMT.2003.nordzee1.v150.170.mat D:INoordwijk 2003lnordzee1_noQCVInst10l1050391834.Tue.Apr.15_07_30_34.GMT.2003.nordzee1.v150.170.mat D:INoordwijk 2003ไnordzee1_noQCVInst10l1050395432.Tue.Apr.15_08_30_32.GMT.2003.nordzee1.v150.170.mat D:Noordwijk 2003ไnordzee1 noQCVInst10l1050399034.Tue.Apr.15 09 30 34.GMT.2003.nordzee1.v150.170.mat D:Woordwijk 2003/nordzee1_noQCVInst10\1050402634.Tue.Apr.15_10_30_34.GMT.2003.nordzee1.v150.170.mat D:INoordwijk 2003lnordzee1_noQCVInst10l1050406234.Tue.Apr.15_11_30_34.GMT.2003.nordzee1.v150.170.mat D:Noordwijk 2003/nordzee1 noQCVnst1011050409834.Tue.Apr.15 1230 34.GMT.2003.nordzee1.v150.170.mat D.Woordwijk 2003lnordzee 1 noOCVnst1011050413434.Tue.Apr 15 13 30 34.GMT 2003 nordzee1 v150.170 mat D:INoordwijk 2003/nordzee1_noQCVInst10l1050413434.Tue.Apr.15_13_30_34.GMT.2003.nordzee 1.v150.170.mat D:INoordwijk 2003/nordzee1_noQC/nst10l1050417034.Tue.Apr.15_14_30_34.GMT.2003.nordzee1.v150.170.mat D:INoordwijk 2003lnordzee1_noQCVInst10l1050424235.Tue.Apr.15_16_30_35.GMT.2003.nordzee1.v150.170.mat D:INoordwijk 2003|nordzee1_noQCVInst10\1050427834.Tue.Apr.15 17 30 34.GMT.2003.nordzee1.v150.170.mat D:Noordwijk 2003/nordzee1 noQCVInst10l1050431434.Tue.Apr.15 18 30 34.GMT.2003.nordzee1.v150.170.mat D:Noordwijk 2003lnordzee1 noQCVInst10l1050435032. Tue.Apr. 15 19 30 32.GMT 2003.nordzee1 v150.170.mat D:Woordwijk 2003lnordzee1 noQCVInst1011050456636.Wed.Apr.16_01 30 36.GMT.2003.nordzee1.v150.170.ma D:INoordwijk 2003lnordzee1 noQCVInst1011050471036.Wed.Apr.16 05 30 36.GMT.2003.nordzee1.v150.170.ma D:Woordwijk 2003lnordzee1_noQClInst10 1050474635.Wed.Apr.16_06_30_35.GMT.2003.nordzee1.v150.170.ma D:Woordwijk 2003ไnordzee1_noQCl|nst10l1050481837.Wed.Apr.16_08_30_37.GMT.2003.nordzee1.v150.170.ma D:INoordwijk 2003lnordzee1_noQClInst10l1050485435.Wed.Apr.16_09_30_35.GMT.2003.nordzee1.v150.170.mat Instrument12

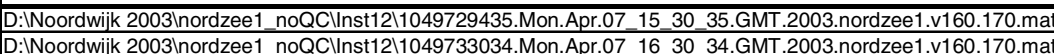
D:Woordwijk 2003lnordzee1_noQC/Inst1211049733034.Mon.Apr.07_16_30_34.GMT.2003.nordzee1.v160.170.mat D:Noordwijk 2003/nordzee1_noQCVnst12l1049736634.Mon.Apr.07_17_30_34.GMT.2003.nordzee1.v160.170.mat \begin{tabular}{l} 
D:INoordwijk 2003lnordzee1_noQCVInst12\1049740235.Mon.Apr.07_18_30_35.GMT.2003.nordzee1.v160.170.mat \\
\hline D:Noordwijk 2003lnordzee1_noQCVnst12\1049743834.Mon.Apr.07 19 30 34.GMT.2003.nordzee1.v160.170.mat
\end{tabular} D:Woordwijk 2003lnordzee1 noQCVInst12l1049765437.Tue.Apr.08 01 30 37.GMT.2003.nordzee1.v160.170.mat D:INoordwijk 2003lnordzee1_noQClInst12\1049779835.Tue.Apr.08_05_30_35.GMT.2003.nordzee1.v160.170.mat D:WNoordwijk 2003ไnordzee1_noQCVInst12\1049783434.Tue.Apr.08_06_30_34.GMT.2003.nordzee1.v160.170.mat

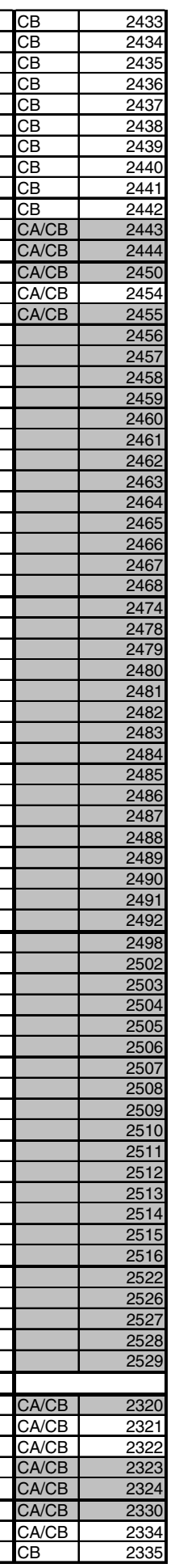


D:INoordwijk 2003/nordzee1_noQCVInst1211049787035.Tue.Apr.08_07_30_35.GMT.2003.nordzee1.v160.170.mat D:INoordwijk 2003Inordzee1_noQCVInst1211049790634.Tue.Apr.08_08 30_34.GMT.2003.nordzee1.v160.170.mat D:INoordwijk 2003\nordzee1_noQCVInst12\1049794235.Tue.Apr.08_09_30_35.GMT.2003.nordzee1.v160.170.mat D:INoordwijk 2003lnordzee1_noQCVInst12\1049797835.Tue.Apr.08_10_30_35.GMT.2003.nordzee1.v160.170.mat D:INoordwijk 2003/nordzee1_noQCVInst12\1049801435.Tue.Apr.08_11_30_35.GMT.2003.nordzee1.v160.170.mat D:INoordwijk 2003\nordzee1_noQCVInst12\1049805035.Tue.Apr.08_12_30_35.GMT.2003.nordzee1.v160.170.mat D:INoordwijk 2003ไnordzee1_noQCVInst12\1049808635.Tue.Apr.08_13_30_35.GMT.2003.nordzee1.v160.170.mat D:Noordwijk 2003/nordzee1 noQCVInst1211049812235.Tue.Apr.08 1430 35.GMT.2003.nordzee1.v160.170.mat D:INoordwijk 2003lnordzee1_noQCl|nst12\1049815834.Tue.Apr.08 15 30 34.GMT.2003.nordzee1.v160.170.mat D:INoordwijk 2003\nordzee1_noQCVInst12\1049819434.Tue.Apr.08_16_30_34.GMT.2003.nordzee1.v160.170.mat D:INoordwijk 2003lnordzee1_noQCVnst1211049823035.Tue.Apr.08_17_30_35.GMT.2003.nordzee1.v160.170.mat D:INoordwijk 2003lnordzee1_noQCVInst1211049826635.Tue.Apr.08_18_30_35.GMT.2003.nordzee1.v160.170.mat D:INoordwijk 2003ไnordzee1_noQCVInst12\1049830234.Tue.Apr.08_19_30_34.GMT.2003.nordzee1.v160.170.mat D:INoordwijk 2003ไnordzee1_noQC|Inst12\1049851837.Wed.Apr.09_01_30_37.GMT.2003.nordzee1.v160.170.mat D:INoordwijk 2003lnordzee1 noQCVInst12l1049866236.Wed.Apr.09 05 30 36.GMT.2003.nordzee1.v160.170.mat D:INoordwijk 2003lnordzee1 noQCVInst12\1049869834.Wed.Apr.09 $06 \quad 30$ 34.GMT.2003.nordzee1.v160.170.mat D:WNoordwijk 2003ไnordzee1_noQCVInst12\1049873434.Wed.Apr.09_07_30_34.GMT.2003.nordzee1.v160.170.mat D:Noordwijk 2003lnordzee1_noQClnst12\1049877034.Wed.Apr.09_08_30_34.GMT.2003.nordzee1.v160.170.mat D:INoordwijk 2003\nordzee1_noQCVInst12l1049880632.Wed.Apr.09_09_30_32.GMT.2003.nordzee1.v160.170.mat D:INoordwijk 2003lnordzee1_noQClInst12l1049884235.Wed.Apr.09_10_30_35.GMT.2003.nordzee1.v160.170.mat D:Noordwijk 2003\nordzee1_noQCVInst12\1049887835.Wed.Apr.09 11 30 35.GMT.2003.nordzee1.v160.170.mat D:INoordwijk 2003\nordzee1_noQCVInst12\1049891434.Wed.Apr.09_12_30_34.GMT.2003.nordzee1.v160.170.mat D:INoordwijk 2003\nordzee1 noQCVInst12l1049895035.Wed.Apr.09 13 30 35.GMT.2003.nordzee1.v160.170.mat D:Noordwijk 2003lnordzee1 noOClnst12l1049898634.Wed Apr.09 $14 \quad 30$ 34.GMT.2003.nordzee1.v160.170.mat D:WNoordwijk 2003 Inordzee1 noQCVInst12\1049902234.Wed.Apr.09 15 30 34.GMT.2003.nordzee1.v160.170.mat D:INoordwijk 2003lnordzee1_noQClInst12l1049905834.Wed.Apr.09_16_30_34.GMT.2003.nordzee1.v160.170.ma D:WNoordwijk 2003lnordzee1_noQC \Inst1211049909434.Wed.Apr.09_17_30_34.GMT.2003.nordzee1.v160.170.mat D:INoordwijk 2003\nordzee1_noQCVInst12\1049913035.Wed.Apr.09_18_30_35.GMT.2003.nordzee1.v160.170.mat D:INoordwijk 2003/nordzee1_noQCVInst12\1049916634.Wed.Apr.09_19_30 34.GMT.2003.nordzee1.v160.170.mat D:WNoordwijk 2003|nordzee1_noQCVInst12〈1049938236.Thu.Apr.10_01_30_36.GMT.2003.nordzee1.v160.170.mat D:INoordwijk 2003ไnordzee1 noQCVInst12\1049952636.Thu.Apr.10 $05 \quad 30 \quad 36$. GMT.2003.nordzee1.v160.170.mat D:INoordwijk 2003/nordzee1_noQCVInst12\1049956234.Thu.Apr.10_06 30_34.GMT.2003.nordzee1.v160.170.mat D:INoordwijk 2003\nordzee1_noQCVInst12〈1049956234.Thu.Apr.10_06_30_34.GMT.2003.nordzee1.v160.170.mat D:INoordwijk 2003\nordzee1_noQCVnst12\1049959835.Thu.Apr.10_07_30_35.GMT.2003.nordzee1.v160.170.mat D:INoordwijk 2003\nordzee1_noQCVInst12\1049963435.Thu.Apr.10_08_30_35.GMT.2003.nordzee1.v160.170.mat D:INoordwijk 2003\nordzee1_noQCVInst12\1049970635.Thu.Apr.10_10_30_35.GMT.2003.nordzee1.v160.170.mat D:INoordwijk 2003\nordzee1_noQCVInst12\1049974231.Thu.Apr.10_11_30_31.GMT.2003.nordzee1.v160.170.mat D:INoordwijk 2003ไnordzee1 noQCVnst12\1049977835.Thu.Apr.10 12 30 35.GMT.2003.nordzee1.v160.170.mat D:INoordwijk 2003/nordzee1_noQCVInst12l1049981435.Thu.Apr.10_13_30_35.GMT.2003.nordzee1.v160.170.mat D:INoordwijk 2003\nordzee1_noQCVInst12\1049985034.Thu.Apr.10_14_30_34.GMT.2003.nordzee1.v160.170.mat D:INoordwijk 2003\nordzee1_noQCl|nst12\1049988635.Thu.Apr.10_15_30_35.GMT.2003.nordzee1.v160.170.mat D:INoordwijk 2003/nordzee1_noQCVInst12\1049992234.Thu.Apr.10_16_30_34.GMT.2003.nordzee1.v160.170.mat D:INoordwijk 2003\nordzee1_noQCVInst12\1049995835.Thu.Apr.10_17_30_35.GMT.2003.nordzee1.v160.170.mat D:INoordwijk 2003\nordzee1_noQCVInst12\1049999435.Thu.Apr.10_18_30_35.GMT.2003.nordzee1.v160.170.mat D:INoordwijk 2003lnordzee1 noQCVInst12 1050003034.Thu.Apr.10 19 30 34.GMT.2003.nordzee1.v160.170.mat D:INoordwijk 2003ไnordzee1_noQCVInst12\1050024636.Fri.Apr.11_01_30_36.GMT.2003.nordzee1.v160.170.mat D:INoordwijk 2003/nordzee1_noQCVInst121050039036.Fri.Apr.11_05 30 36.GMT.2003.nordzee1.v160.170.mat D:INoordwijk 2003lnordzee1_noQCVInst12l1050042635.Fri.Apr.11_06_30_35.GMT.2003.nordzee1.v160.170.ma D:INoordwijk 2003\nordzee1_noQCVInst12\1050046234.Fri.Apr.11_07_30_34.GMT.2003.nordzee1.v160.170.ma D:WNoordwijk 2003lnordzee1_noQCVInst12\1050049834.Fri.Apr.11_08_30_34.GMT.2003.nordzee1.v160.170.mat D:INoordwijk 2003ไnordzee1_noQCVInst12\1050053434.Fri.Apr.11_09_30_34.GMT.2003.nordzee1.v160.170.mat D:INoordwijk 2003lnordzee1_noQCVInst1211050057035.Fri.Apr.11_10_30_35.GMT.2003.nordzee1.v160.170.mat D:INoordwijk 2003lnordzee1 noQCVnst12l1050060634.Fri.Apr.11 11 30 34.GMT.2003.nordzee1.v160.170.mat D:INoordwijk 2003ไnordzee1 noQCVnst12\1050064231.Fri.Apr.11 1230 31.GMT.2003.nordzee1.v160.170.mat

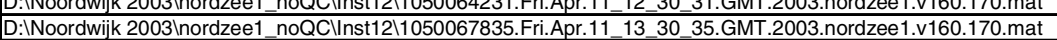
D:INoordwijk 2003\nordzee1_noQCVInst12\1050071435.Fri.Apr.11_14_30_35.GMT.2003.nordzee1.v160.170.mat D:INoordwijk 2003/nordzee1_noQCVnst12\1050075034.Fri.Apr.11_15_30_34.GMT.2003.nordzee1.v160.170.mat D:INoordwijk 2003\nordzee1_noQCVnst12\1050078635.Fri.Apr.11_16_30_35.GMT.2003.nordzee1.v160.170.mat D:INoordwijk 2003lnordzee1_noQCVInst12l1050082234.Fri.Apr.11_17_30_34.GMT.2003.nordzee1.v160.170.mat D:INoordwijk 2003ไnordzee1_noQCVnst12\1050085835.Fri.Apr.11_18_30_35.GMT.2003.nordzee1.v160.170.mat D:INoordwijk 2003ไnordzee1_noQCVInst12l1050089434.Fri.Apr.11_19_30_34.GMT.2003.nordzee1.v160.170.mat D:WNoordwijk 2003lnordzee1_noQCVInst12\1050111036.Sat.Apr.12_01_30_36.GMT.2003.nordzee1.v160.170.mat D:INoordwijk 2003\nordzee1_noQCVInst12\1050125436.Sat.Apr.12_05_30_36.GMT.2003.nordzee1.v160.170.mat D:INoordwijk 2003\nordzee1_noQClnnst12\1050129035.Sat.Apr.12_06_30_35.GMT.2003.nordzee1.v160.170.mat D:INoordwijk 2003lnordzee1_noQCVInst12\1050132634.Sat.Apr.12_07_30_34.GMT.2003.nordzee1.v160.170.mat D:INoordwijk 2003lnordzee1_noQCVInst12\1050136235.Sat.Apr.12_08_30_35.GMT.2003.nordzee1.v160.170.mat D:INoordwijk 2003\nordzee1_noQCVInst12\1050139835.Sat.Apr.12_09_30_35.GMT.2003.nordzee1.v160.170.mat D:INoordwijk 2003ไnordzee1 noQCVInst12\1050143435.Sat.Apr.12 10 30 35.GMT.2003.nordzee1.v160.170.mat D:INoordwijk 2003lnordzee1_noQCVInst12\1050147034.Sat.Apr.12_11_30_34.GMT.2003.nordzee1.v160.170.mat D:INoordwijk 2003ไnordzee1_noQCVnst12\1050150634.Sat.Apr.12_12 30 34.GMT.2003.nordzee1.v160.170.mat D:INoordwijk 2003\nordzee1_noQCVInst12\1050154235.Sat.Apr.12_13_30_35.GMT.2003.nordzee1.v160.170.mat D:INoordwijk 2003ไnordzee1_noQCVInst12\1050157835.Sat.Apr.12_14_30_35.GMT.2003.nordzee1.v160.170.mat D:INoordwijk 2003lnordzee1_noQCl|nst12\1050161434.Sat.Apr.12_15_30_34.GMT.2003.nordzee1.v160.170.mat D:INoordwijk 2003ไnordzee1_noQCVInst12\1050165034.Sat.Apr.12_16_30_34.GMT.2003.nordzee1.v160.170.mat

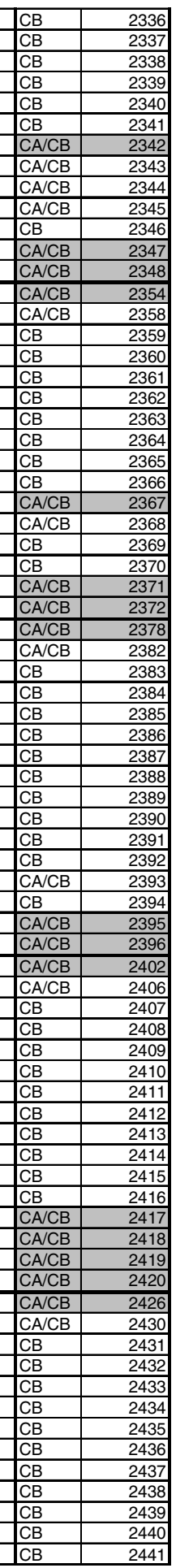


D:INoordwijk 2003Inordzee1_noQCVInst1211050168634.Sat.Apr.12_17_30_34.GMT.2003.nordzee1.v160.170.mat D:Woordwijk 2003/nordzee1 noOCVnst12l1050172234.Sat.Apr.12 1830 34.GMT 2003 nordzee1 v160.170.mat D:INoordwijk 2003ไnordzee1_noQCVInst12\1050175835.Sat.Apr.12_19_30_35.GMT.2003.nordzee1.v160.170.mat D:Woordwijk 2003lnordzee1_noQCVInst12〈1050197437.Sun.Apr.13_01_30_37.GMT.2003.nordzee1.v160.170.mat D:INoordwijk 2003lnordzee1_noQCVInst12\1050211836.Sun.Apr.13_05_30_36.GMT.2003.nordzee1.v160.170.mat D:INoordwijk 2003\nordzee1_noQCVInst12\1050215434.Sun.Apr.13_06_30_34.GMT.2003.nordzee1.v160.170.mat D:Woordwijk 2003ไnordzee1 noQCVInst12\1050219035.Sun.Apr.13 07 30 35.GMT.2003.nordzee1.v160.170.mat D:INoordwijk 2003lnordzee1_noQCVInst12\1050222634.Sun.Apr.13 08 30 34.GMT.2003.nordzee1.v160.170.mat D:Noordwijk 2003lnordzee1_noQCVInst12l1050226235.Sun.Apr.13 09 30 35.GMT.2003.nordzee1.v160.170.mat D::Noordwijk 2003/nordzee1_noQC/nst12l1050226235.Sun.Apr.13_09_30_35.GMT.2003.nordzee1.v160.170.mat D:INoordwijk 2003ไnordzee1_noQCVInst12\1050229835.Sun.Apr.13_10_30_35.GMT.2003.nordzee1.v160.170.mat D:Woordwijk 2003lnordzee1_noQCVInst12\1050237034.Sun.Apr.13_12_30_34.GMT.2003.nordzee1.v160.170.mat D:INoordwijk 2003lnordzee1_noQCVInst12\1050240634.Sun.Apr.13_13_30_34.GMT.2003.nordzee1.v160.170.mat D:Woordwijk 2003lnordzee1_noQCVInst12\1050244234.Sun.Apr.13_14_30_34.GMT.2003.nordzee1.v160.170.mat D:INoordwijk 2003lnordzee1 noQCVInst12l1050247834.Sun.Apr.13 15 30 34.GMT.2003.nordzee1.v160.170.mat D:INoordwijk 2003!nordzee1 noQCVInst12\1050251435.Sun.Apr.13 16 30 35.GMT.2003.nordzee1.v160.170.mat D:Woordwijk 2003/nordzee1 noQCVInst12\1050255035.Sun.Apr.13 17 30 35.GMT.2003.nordzee1.v160.170.mat D:Woordwijk 2003lnordzee1_noQClnst1211050258634.Sun.Apr.13_18_30_34.GMT.2003.nordzee1.v160.170.mat D:Noordwijk 2003lnordzee1_noQClInst12\1050262234.Sun.Apr.13_19_30_34.GMT.2003.nordzee1.v160.170.mat D:INoordwijk 2003/nordzee1_noQCVInst1211050283837.Mon.Apr.14_01_30_37.GMT.2003.nordzee1.v160.170.mat D:Woordwijk 2003/nordzee1_noQCVInst12\1050298236.Mon.Apr.14_05_30_36.GMT.2003.nordzee1.v160.170.mat D:INoordwijk 2003lnordzee1_noQCVInst12l1050301835.Mon.Apr.14_06_30_35.GMT.2003.nordzee1.v160.170.mat D:Noordwijk 2003lnordzee1_noQCVInst12l1050305434.Mon.Apr.14 07 30 34.GMT.2003.nordzee1.v160.170.mat D:INoordwijk 2003lnordzee1_noQCVInst12\1050309034.Mon.Apr.14_08_30_34.GMT.2003.nordzee1.v160.170.mat D:Noordwijk 2003/nordzee1_noQC Inst12l1050309034.Mon.Apr.14_08_30_34.GMT.2003.nordzee1.v160.170.mat

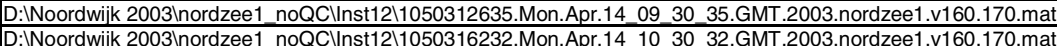
D:INoordwijk 2003lnordzee1_noQCVInst12\1050319835.Mon.Apr.14_11_30_35.GMT.2003.nordzee1.v160.170.mat D:Woordwijk 2003lnordzee1_noQCVInst12l1050323434.Mon.Apr.14_12_30_34.GMT.2003.nordzee1.v160.170.mat D:INoordwijk 2003lnordzee1_noQCVInst12\1050327035.Mon.Apr.14_13_30_35.GMT.2003.nordzee1.v160.170.mat D:INoordwijk 2003ไnordzee1 noQCVInst12l1050330634.Mon.Apr.14 14 30 34.GMT.2003.nordzee1.v160.170.mat D:Woordwijk 2003lnordzee1_noQCVInst12l1050334234.Mon.Apr.14_15_30_34.GMT.2003.nordzee1.v160.170.ma D:INoordwijk 2003lnordzee1_noQClInst12\1050337834.Mon.Apr.14_16_30_34.GMT.2003.nordzee1.v160.170.mat D:Noordwijk 2003lnordzee1_noQCVInst12\1050341434.Mon.Apr.14_17_30_34.GMT.2003.nordzee1.v160.170.ma D:Noordwijk 2003lnordzee1_noQCVInst12\1050345034.Mon.Apr.14_18_30_34.GMT.2003.nordzee1.v160.170.ma D:INoordwijk 2003/nordzee1_noQCVInst12\1050348634.Mon.Apr.14_19_30_34.GMT.2003.nordzee1.v160.170.mat D:INoordwijk 2003lnordzee1_noQCVInst12\1050370237.Tue.Apr.15_01_30_37.GMT.2003.nordzee1.v160.170.mat D:INoordwijk 2003lnordzee1_noQCVInst12\1050384636.Tue.Apr.15_05_30_36.GMT.2003.nordzee1.v160.170.mat D:INoordwijk 2003lnordzee1_noQCVInst12\1050388234.Tue.Apr.15_06_30_34.GMT.2003.nordzee1.v160.170.mat D:INoordwijk 2003ไnordzee1_noQClInst12\1050391834.Tue.Apr.15_07_30_34.GMT.2003.nordzee1.v160.170.mat D:INoordwijk 2003/nordzee1_noQCVInst12l1050395432.Tue.Apr.15_08_30_32.GMT.2003.nordzee1.v160.170.mat D:INoordwijk 2003ไnordzee1_noQCVInst12l1050399034.Tue.Apr.15_09_30_34.GMT.2003.nordzee1.v160.170.mat D:INoordwijk 2003lnordzee1_noQCVInst12\1050402634.Tue.Apr.15_10_30_34.GMT.2003.nordzee1.v160.170.mat D:INoordwijk 2003lnordzee1_noQCVInst12\1050406234.Tue.Apr.15_11_30_34.GMT.2003.nordzee1.v160.170.mat D:WNoordwijk 2003lnordzee1_noQCVInst12\1050409834.Tue.Apr.15_12_30_34.GMT.2003.nordzee1.v160.170.mat D:Noordwijk 2003lnordzee1 noQCVnst12l1050413434.Tue.Apr.15 13 30 34.GMT.2003.nordzee1.v160.170.mat D:Woordwijk 2003lnordzee1 noQCVInst12l1050417034.Tue.Apr.15 14 30 34.GMT.2003.nordzee1.v160.170.mat D:Noordwijk 2003lnordzee1 noQCVInst12\1050420634.Tue.Apr.15 15 30 34.GMT.2003.nordzee1.v160.170.mat D:INoordwijk 2003ไnordzee1_noQCVInst12\1050424235.Tue.Apr.15_16_30_35.GMT.2003.nordzee1.v160.170.mat D:INoordwijk 2003lnordzee1_noQCVInst12l1050427834.Tue.Apr.15_17_30_34.GMT.2003.nordzee1.v160.170.mat D:Noordwijk 2003/nordzee1 noQCVInst1211050431434.Tue.Apr.15 1830 34.GMT.2003.nordzee1.v160.170.mat D:INoordwijk 2003ไnordzee1_noQCVInst12\1050435032.Tue.Apr.15_19_30_32.GMT.2003.nordzee1.v160.170.mat D:Woordwijk 2003lnordzee1 noQCVInst12l1050456636.Wed.Apr.16 01 30 36.GMT.2003.nordzee1.v160.170.mat D:Woordwijk 2003lnordzee1 noQCVInst1211050471036.Wed.Apr.16 05 30 36.GMT.2003.nordzee1.v160.170.ma D:INoordwijk 2003ไnordzee1_noQClInst12\1050474635.Wed.Apr.16_06_30_35.GMT.2003.nordzee1.v160.170.ma D:Woordwijk 2003lnordzee1_noQClInst12\1050481837.Wed.Apr.16_08_30_37.GMT.2003.nordzee1.v160.170.ma D:INoordwijk 2003lnordzee1_noQCVInst12\1050485435.Wed.Apr.16_09_30_35.GMT.2003.nordzee1.v160.170.mat Instrument13

D:Woordwijk 2003ไnordzee1 noQCVInst13\1049729435.Mon.Apr.07 15 30 35.GMT.2003.nordzee1.v170.170.mat D:Noordwijk 2003lnordzee1 noQCVInst13l1049733034.Mon.Apr.07 $16 \quad 30$ 34.GMT.2003.nordzee1.v170.170.mat D:INoordwijk 2003lnordzee1 noQCVInst13|1049736634.Mon.Apr.07 17 30 34.GMT.2003.nordzee1.v170.170.mat

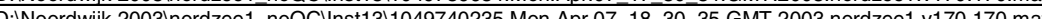
D:Woordwijk 2003lnordzee1_noQCVInst13\1049743834.Mon.Apr.07_19_30_34.GMT.2003.nordzee1.v170.170.mat D:Woordwijk 2003lnordzee1_noQCVnst13l1049765437.Tue.Apr.08_01_30_37.GMT.2003.nordzee1.v170.170.mat D:INoordwijk 2003|nordzee1_noQCVInst13\1049779835.Tue.Apr.08 05 30 35.GMT.2003.nordzee1.v170.170.mat D:INoordwijk 2003/nordzee1 noQCVInst1311049783434.Tue.Apr.08 $06 \quad 30$ 34.GMT 2003 nordzee1 v170.170 mat D:INoordwijk 2003/nordzee1 noQCVInst13\1049787035.Tue.Apr.08 07 30 35.GMT.2003.nordzee1.v170.170.mat D:INoordwijk 2003lnordzee1 noQCVInst13l1049790634.Tue.Apr.08 08 30 34.GMT.2003.nordzee1.v170.170.mat D.Noordwijk 2003lnordzee1 noQCVInst13\1049794235.Tue.Apr.08 09 30 35.GMT.2003.nordzee1.v170.170.mat D:INoordwijk 2003/nordzee1_noQCVInst13/1049794235.Tue.Apr.08_09_30_35.GMT.2003.nordzee1.v170.170.mat D:Woordwijk 2003/nordzee1_noQCVInst13l1049797835.Tue.Apr.08_10_30_35.GMT.2003.nordzee1.v170.170.mat D:INoordwijk 2003ไnordzee1_noQCVInst13\1049801435.Tue.Apr.08_11_30_35.GMT.2003.nordzee1.v170.170.mat D:INoordwijk 2003lnordzee1 noQCVInst13|1049808635.Tue.Apr.08_13 30 35.GMT.2003.nordzee1.v170.170.mat D:INoordwijk 2003|nordzee1_noQCVInst13\1049812235.Tue.Apr.08_14_30_35.GMT.2003.nordzee1.v170.170.mat D:Woordwijk 2003ไnordzee1_noQCVInst13\1049815834.Tue.Apr.08_15_30_34.GMT.2003.nordzee1.v170.170.mat

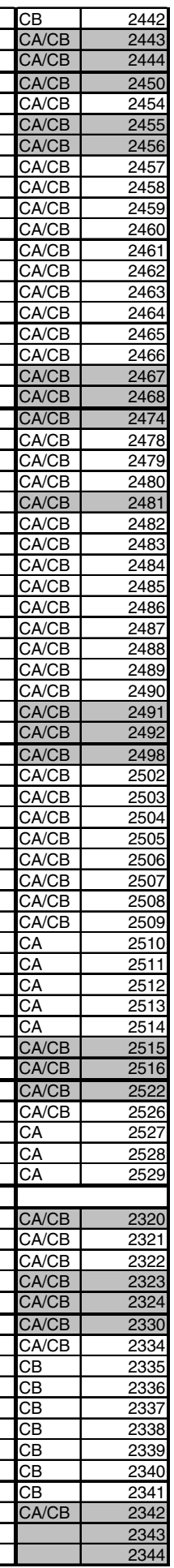




\begin{tabular}{|c|c|}
\hline D:INoordwijk 2003ไnordzee1_noQC\Inst13\1049819434.Tue.Apr.08_16_30_34.GMT.2003.nordzee1.v170.17 & 2345 \\
\hline D:Woordwijk 2003lnordzee1_noQClInst13\1049823035.Tue.Apr.08_17_30_35.GMT.2003.nordzee1.v170.170.mat & 2346 \\
\hline D:Woordwijk 2003ไnordzee1_noQCVnst13\1049826635.Tue.Apr.08_18_30_35.GMT.2003.nordzee1.v170.170.mat & 2347 \\
\hline D:Woordwijk 2003ไnordzee1_noQCVInst13\1049830234.Tue.Apr.08_19_30_34.GMT.2003.nordzee1.v170.170.mat & 2348 \\
\hline D:Woordwijk 2003ไnordzee1_noQC\Inst13\1049851837.Wed.Apr.09_01_30_37.GMT.2003.nordzee1.v170.170.mat & \\
\hline D:Woordwijk 2003ไnordzee1_noQCVInst13\1049866236.Wed.Apr.09_05_30_36.GMT.2003.nordzee1.v170.170.mat & 2358 \\
\hline D:Woordwijk 2003ไnordzee1_noQC\Inst13\1049869834.Wed.Apr.09_06 30 34.GMT.2003.nordzee1.v170.170.mat & 2359 \\
\hline D:Woordwijk 2003lnordzee1_noQCVInst13\1049873434.Wed.Apr.09_07_30_34.GMT.2003.nordzee1.v170.170.mat & 2360 \\
\hline D:Woordwijk 2003|nordzee1_noQCVInst1311049877034.Wed.Apr.09_08_30_34.GMT.2003.nordzee1.v170.170.mat & \\
\hline D:Woordwijk 2003ไnordzee1_noQC\Inst13\1049880632.Wed.Apr.09_09_30_32.GMT.2003.nordzee1.v170.170.mat & 2362 \\
\hline D:Woordwijk 2003ไnordzee1_noQC\Inst13\1049884235.Wed.Apr.09_10_30_35.GMT.2003.nordzee1.v170.170.mat & 2363 \\
\hline D:Woordwijk 2003ไnordzee1_noQC\Inst13\1049887835.Wed.Apr.09_11_30_35.GMT.2003.nordzee1.v170.170.mat & 2364 \\
\hline D:Woordwijk 2003ไnordzee1_noQC\Inst13\1049891434.Wed.Apr.09_12_30_34.GMT.2003.nordzee1.v170.170.mat & \\
\hline D:Woordwijk 2003ไnordzee1_noQC\Inst13\1049895035.Wed.Apr.09_13_30_35.GMT.2003.nordzee1.v170.170.mat & 2366 \\
\hline D:Noordwijk 2003\nordzee1_noQCVInst13|1049898634.Wed.Apr.09_14_30_34.GMT.2003.nordzee1.v170.170.mat & 2367 \\
\hline D:Woordwijk 2003ไnordzee1_noQCVInst13|1049902234.Wed.Apr.09_15_30_34.GMT.2003.nordzee1.v170.170.mat & \\
\hline D:Woordwijk 2003ไnordzee1_noQC\Inst13\1049905834.Wed.Apr.09_16_30_34.GMT.2003.nordzee1.v170.170.mat & 2369 \\
\hline D:Woordwijk 2003ไnordzee1_noQC\Inst13\1049909434.Wed.Apr.09_17_30_34.GMT.2003.nordzee1.v170.170.mat & 2370 \\
\hline D:INoordwijk 2003lnordzee1_noQCVInst13\1049913035.Wed.Apr.09_18_30_35.GMT.2003.nordzee1.v170.170.mat & 2371 \\
\hline D:Woordwijk 2003ไnordzee1_noQC\Inst13\1049916634.Wed.Apr.09_19_30_34.GMT.2003.nordzee1.v170.170.mat & \\
\hline D:Woordwijk 2003ไnordzee1_noQCVInst13|1049938236.Thu.Apr.10_01_30_36.GMT.2003.nordzee1.v170.170.mat & 2378 \\
\hline D:Woordwijk 2003ไnordzee1_noQCVInst13\1049952636.Thu.Apr.10_05_30_36.GMT.2003.nordzee1.v170.170.mat & 2382 \\
\hline D:Woordwijk 2003ไnordzee1_noQCVInst13\1049956234.Thu.Apr.10_06_30_34.GMT.2003.nordzee1.v170.170.mat & \\
\hline D:Woordwijk 2003ไnordzee1_noQCVInst13\1049959835.Thu.Apr.10_07_30_35.GMT.2003.nordzee1.v170.170.mat & \\
\hline D:Noordwijk 2003lnordzee1_noQCVInst13\1049963435.Thu.Apr.10_08_30_35.GMT.2003.nordzee1.v170.170.mat & 2385 \\
\hline D:Woordwijk 2003lnordzee1_noQCVInst13\1049967034.Thu.Apr.10_09_30_34.GMT.2003.nordzee1.v170.170.mat & 2386 \\
\hline D:Woordwijk 2003lnordzee1_noQCVInst13\1049970635.Thu.Apr.10_10_30_35.GMT.2003.nordzee1.v170.170.mat & 2387 \\
\hline D:Woordwijk 2003ไnordzee1_noQCVnst13\1049974231.Thu.Apr.10_11_30_31.GMT.2003.n & \\
\hline D:Woordwijk 2003ไnordzee1_noQCVInst13\1049977835.Thu.Apr.10_12_30_35.GMT.2003.nordzee1.v170.170.mat & 2389 \\
\hline D:Woordwijk 2003ไnordzee1_noQCVInst13\1049981435.Thu.Apr.10_13_30_35.GMT.2003.nordzee1.v170.170.mat & 2390 \\
\hline D:Woordwijk 2003lnordzee1_noQCVInst13\1049985034.Thu.Apr.10_14_30_34.GMT.2003.nordzee1.v170.170.mat & \\
\hline D:Woordwijk 2003lnordzee1_noQCVInst13\1049988635.Thu.Apr.10_15_30_35.GMT.2003.nordzee1.v170.170.mat & 2392 \\
\hline D:Noordwijk 2003lnordzee1_noQClinst13l1049992234.Thu.Apr.10_16_30_34.GMT.2003.nordzee1.v170.170.mat & 2393 \\
\hline D:INoordwijk 2003lnordzee1_noQCVInst13\1049995835.Thu.Apr.10_17_30_35.GMT.2003.nordzee1.v170.170.mat & 2394 \\
\hline D:INoordwijk 2003lnordzee1_noQCVInst13\1049999435.Thu.Apr.10_18_30_35.GMT.2003.nordzee1.v170.170.mat & 2395 \\
\hline D:WNoordwijk 2003ไnordzee1_noQC\Inst13\1050003034.Thu.Apr.10_19_30_34.GMT.2003.nordzee1.v170.170.mat & 2396 \\
\hline D:Woordwijk 2003lnordzee1_noQCVInst13\1050024636.Fri.Apr.11_01_30_36.GMT.2003.nordzee1.v170.170.mat & 2402 \\
\hline D:INoordwijk 2003lnordzee1_noQCVInst13\1050039036.Fri.Apr.11_05_30_36.GMT.2003.nordzee1.v170.170.mat & 2406 \\
\hline D:INoordwijk 2003\nordzee1_noQCVInst13\1050042635.Fri.Apr.11_06_30_35.GMT.2003.nordzee1.v170.170.mat & 2407 \\
\hline D:INoordwijk 2003ไnordzee1_noQCVInst13\1050046234.Fri.Apr.11_07_30_34.GMT.2003.nordzee1.v170.170.mat & 2408 \\
\hline D:INoordwijk 2003lnordzee1_noQCVInst1311050049834.Fri.Apr.11_08_30_34.GMT.2003.nordzee1.v170.170.mat & 2409 \\
\hline D:INoordwijk 2003ไnordzee1_noQCVInst13\1050053434.Fri.Apr.11_09_30_34.GMT.2003.nordzee1.v170.170.mat & 2410 \\
\hline D:INoordwijk 2003ไnordzee1_noQCVInst13\1050057035.Fri.Apr.11_10_30_35.GMT.2003.nordzee1.v170.170.mat & 2411 \\
\hline D:INoordwijk 2003\nordzee1_noQC|Inst13\1050060634.Fri.Apr.11_11_30_34.GMT.2003.nordzee1.v170.170.mat & 2412 \\
\hline D:INoordwijk 2003ไnordzee1_noQCVInst13\1050064231.Fri.Apr.11_12_30_31.GMT.2003.nordzee1.v170.170.mat & 2413 \\
\hline D:INoordwijk 2003ไnordzee1_noQCVInst13\1050067835.Fri.Apr.11_13_30_35.GMT.2003.nordzee1.v170.170.mat & 2414 \\
\hline D:INoordwijk 2003ไnordzee1_noQCVInst13\1050071435.Fri.Apr.11_14_30_35.GMT.2003.nordzee1.v170.170.mat & 2415 \\
\hline D:INoordwijk 2003lnordzee1_noQCVInst13\1050075034.Fri.Apr.11_15_30_34.GMT.2003.nordzee1.v170.170.mat & 2416 \\
\hline D:INoordwijk 2003|nordzee1_noQC|Inst13\1050078635.Fri.Apr.11_16_30_35.GMT.2003.nordzee1.v170.170.mat & 2417 \\
\hline D:INoordwijk 2003/nordzee1_noQCVInst13\1050082234.Fri.Apr.11_17_30_34.GMT.2003.nordzee1.v170.170.mat & 2418 \\
\hline D:INoordwijk 2003|nordzee1_noQC|Inst13\1050085835.Fri.Apr.11_18_30_35.GMT.2003.nordzee1.v170.170.mat & 2419 \\
\hline D:Noordwijk 2003ไnordzee1_noQCVInst13\1050089434.Fri.Apr.11_19_30_34.GMT.2003.nordzee1.v170.170.mat & 2420 \\
\hline D:INoordwijk 2003/nordzee1_noQCVInst1311050111036.Sat.Apr.12_01_30_36.GMT.2003.nordzee1.v170.170.mat & 2426 \\
\hline D:Woordwijk 2003lnordzee1_noQC\Inst13\1050125436.Sat.Apr.12_05_30_36.GMT.2003.nordzee1.v170.170.mat & 2430 \\
\hline D:INoordwijk 2003\nordzee1_noQCVInst13|1050129035.Sat.Apr.12_06_30_35.GMT.2003.nordzee1.v170.170.mat & 2431 \\
\hline D:INoordwijk 2003/nordzee1_noQCVInst13|1050132634.Sat.Apr.12_07_30_34.GMT.2003.nordzee1.v170.170.mat & \\
\hline D:INoordwijk 2003\nordzee1_noQCVInst13\1050136235.Sat.Apr.12_08_30_35.GMT.2003.nordzee1.v170.170.mat & 2433 \\
\hline D:Noordwijk 2003lnordzee1_noQCVInst13\1050139835.Sat.Apr.12_09_30_35.GMT.2003.nordzee1.v170.170.mat & 2434 \\
\hline D:INoordwijk 2003/nordzee1_noQCVInst13\1050143435.Sat.Apr.12_10_30_35.GMT.2003.nordzee1.v170.170.mat & 2435 \\
\hline D:INoordwijk 2003/nordzee1_noQCVInst13|1050147034.Sat.Apr.12_11_30_34.GMT.2003.nordzee1.v170.170.mat & 2436 \\
\hline D:Noordwijk 2003lnordzee1_noQC|Inst13\1050150634.Sat.Apr.12_12_30_34.GMT.2003.nordzee1.v170.170.mat & 2437 \\
\hline D:Noordwijk 2003lnordzee1_noQCVInst13\1050154235.Sat.Apr.12_13_30_35.GMT.2003.nordzee1.v170.170.mat & 2438 \\
\hline D:Woordwijk 2003lnordzee1_noQC\Inst13\1050157835.Sat.Apr.12_14_30_35.GMT.2003.nordzee1.v170.170.mat & 2439 \\
\hline D:Woordwijk 2003lnordzee1_noQC\Inst13\1050161434.Sat.Apr.12_15_30_34.GMT.2003.nordzee1.v170.170.mat & \\
\hline D:Woordwijk 2003lnordzee1_noQC|Inst13\1050165034.Sat.Apr.12_16_30_34.GMT.2003.nordzee1.v170.170.mat & 2441 \\
\hline D:INoordwijk 2003/nordzee1_noQCVInst13|1050168634.Sat.Apr.12_17_30_34.GMT.2003.nordzee1.v170.170.mat & 2442 \\
\hline D:INoordwijk 2003\nordzee1_noQCVInst13|1050172234.Sat.Apr.12_18_30_34.GMT.2003.nordzee1.v170.170.mat & \\
\hline D:Noordwijk 2003lnordzee1_noQC\Inst13\1050175835.Sat.Apr.12_19_30_35.GMT.2003.nordzee1.v170.170.mat & 2444 \\
\hline D:Woordwijk 2003lnordzee1_noQCVInst13\1050197437.Sun.Apr.13_01_30_37.GMT.2003.nordzee1.v170.170.mat & 2450 \\
\hline D:INoordwijk 2003|nordzee1_noQCVInst13|1050211836.Sun.Apr.13_05_30_36.GMT.2003.nordzee1.v170.170.mat & 2454 \\
\hline D:Woordwijk 2003lnordzee1_noQClInst13\1050215434.Sun.Apr.13_06_30_34.GMT.2003.nordzee1.v170.170.mat & 245 \\
\hline D:Noordwijk 2003|nordzee1_noQCl|nst13l1050219035.Sun.Apr.13_07_30_35.GMT.2003.nordzee1.v170.170.mat & 2456 \\
\hline D:Noordwijk 2003ไnordzee1_noQCVInst13ไ10 & \\
\hline D:INoordwijk 2003/nordzee1_noQCVInst13\1050226235.Sun.Apr.13_09_30_35.GMT.2003.nordzee1.v170.170.r & \\
\hline
\end{tabular}


D:INoordwijk 2003lnordzee1_noQCVInst1311050229835.Sun.Apr.13_10_30_35.GMT.2003.nordzee1.v170.170.mat D.Noordwijk 2003lnordzee1 noOCVnst13l1050233432 Sun Apr.13 11 30 32.GMT 2003.nordzee1.v170.170.mat D:INoordwijk 2003ไnordzee1_noQCVInst13\1050237034.Sun.Apr.13_12_30_34.GMT.2003.nordzee1.v170.170.mat D:INoordwijk 2003lnordzee1 noQCVnst13\1050240634.Sun.Apr.13 13 30 34.GMT.2003.nordzee1.v170.170.mat D:INoordwijk 2003\nordzee1_noQCVInst13\1050244234.Sun.Apr.13_14_30_34.GMT.2003.nordzee1.v170.170.mat D:INoordwijk 2003\nordzee1_noQCVInst13\1050247834.Sun.Apr.13_15_30_34.GMT.2003.nordzee1.v170.170.mat D:INoordwijk 2003ไnordzee1_noQCVInst13\1050251435.Sun.Apr.13_16_30_35.GMT.2003.nordzee1.v170.170.mat D:Noordwijk 2003ไnordzee1 noQCVInst13\1050255035.Sun.Apr.13 17 30 35.GMT.2003.nordzee1.v170.170.mat D.WNordwijk 2003 D:INoordwijk 2003/nordzee1_noQCVInst13\1050262234.Sun.Apr.13_19_30_34.GMT.2003.nordzee1.v170.170.mat D:INoordwijk 2003\nordzee1_noQC/nst13\1050262234.Sun.Apr.13_19_30_34.GMT.2003.nordzee1.v170.170.ma D:INoordwijk 2003lnordzee1_noQCไInst13l1050298236.Mon.Apr.14_05_30_36.GMT.2003.nordzee1.v170.170.ma D:INoordwiik 2003/nordzee1 noQCVnst13l1050301835.Mon.Apr.14 $06 \quad 30$ 35.GMT.2003.nordzee1.v170.170.ma D:INoordwijk 2003\nordzee1 noQCVInst13\1050305434.Mon.Apr.14 07 30 34.GMT.2003.nordzee1.v170.170.mat D:INoordwijk 2003ไnordzee1 noQCVnst13\1050309034.Mon.Apr.14 08 30 34.GMT.2003.nordzee1.v170.170.mat D:Noordwijk 2003 nordzee1 noOCInst13\1050312635.Mon Apr.140930 35.GMT.2003 nordzee1.v170.170.mat D:Noordwijk 2003lnordzee1 noQCVInst1311050316232.Mon.Apr.14 1030 32.GMT.2003.nordzee1.v170.170.mat D:INoordwijk 2003/nordzee1_noQC \nst13|1050316232.Mon.Apr.14_10_30_32.GMT.2003.nordzee1.v170.170.mat D:INoordwijk 2003\nordzee1_noQCVnst13\1050319835.Mon.Apr.14_11_30_35.GMT.2003.nordzee1.v170.170.mat D:INoordwijk 2003ไnordzee1_noQCVnst13ไ1050323434.Mon.Apr.14_12_30_34.GMT.2003.nordzee1.v170.170.ma D:INoordwijk 2003\nordzee1_noQCVInst13\1050327035.Mon.Apr.14_13_30_35.GMT.2003.nordzee1.v170.170.mat D:INoordwijk 2003ไnordzee1_noQCVInst13|1050330634.Mon.Apr.14_14_30_34.GMT.2003.nordzee1.v170.170.mat D:INoordwijk 2003ไnordzee1 noQCVnst13|1050334234.Mon.Apr.14 15 30 34.GMT.2003.nordzee1.v170.170.mat D:INoordwijk 2003ไnordzee1 noQCVnst1311050337834.Mon.Apr.14 163034 .GMT 2003 nordzee1 v170.170.mat D:INoordwijk 2003/nordzee1 noQCVInst13\1050341434.Mon.Apr.14 17 30 34.GMT.2003.nordzee1.v170.170.mat D:Noordwijk 2003inordzee1_noQCVInst1311050341434.Mon.Apr.14_17_30_34.GMT.2003.nordzee1.v170.170.mat D:INoordwijk 2003\nordzee1_noQCVnst13\1050345034.Mon.Apr.14_18_30_34.GMT.2003.nordzee1.v170.170.mat D:INoordwijk 2003ไnordzee1 noQCVInst13/1050370237.Tue.Apr.15 01 30 37.GMT.2003.nordzee1.v170.170.mat D:INoordwijk 2003\nordzee1_noQCVnst13\1050384636.Tue.Apr.15_05_30_36.GMT.2003.nordzee1.v170.170.mat D:INoordwijk 2003/nordzee1 noQCVInst13\1050388234.Tue.Apr.15 06 30 34.GMT.2003.nordzee1.v170.170.mat

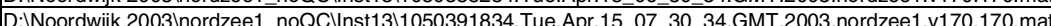

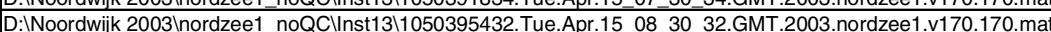
D:INoordwijk 2003/nordzee1_noQC Inst13|1050395432.Tue.Apr.15_08_30_32.GMT.2003.nordzee1.v170.170.mat D:INoordwijk 2003ไnordzee1_noQCVInst13\1050399034.Tue.Apr.15_09_30_34.GMT.2003.nordzee1.v170.170.mat D:INoordwijk 2003ไnordzee1_noQCVInst13\1050402634.Tue.Apr.15_10_30_34.GMT.2003.nordzee1.v170.170.mat D:INoordwijk 2003/nordzee1_noQCVnst13\1050406234.Tue.Apr.15_11_30_34.GMT.2003.nordzee1.v170.170.mat D:INoordwijk 2003ไnordzee1_noQCVInst13l1050409834.Tue.Apr.15_12_30_34.GMT.2003.nordzee1.v170.170.mat D:INoordwijk 2003\nordzee1_noQCVnst13\1050413434.Tue.Apr.15_13_30_34.GMT.2003.nordzee1.v170.170.mat D:INoordwijk 2003ไnordzee1_noQCVInst13\1050417034.Tue.Apr.15_14_30 34.GMT.2003.nordzee1.v170.170.mat D:INoordwijk 2003ไnordzee1 noQClInst13\1050420634.Tue.Apr.15 15 30 34.GMT.2003.nordzee1.v170.170.mat

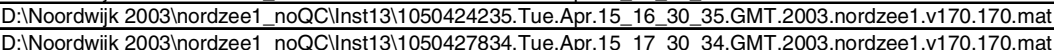
D:INoordwijk 2003ไnordzee1_noQCVnst13\1050427834.Tue.Apr.15_17_30_34.GMT.2003.nordzee1.v170.170.mat D:INoordwijk 2003\nordzee1_noQCVInst13\1050431434.Tue.Apr.15_18_30_34.GMT.2003.nordzee1.v170.170.mat D:INoordwijk 2003\nordzee1_noQCVInst13\1050435032.Tue.Apr.15_19_30_32.GMT.2003.nordzee1.v170.170.ma D:INoordwijk 2003lnordzee1_noQC/nst13/1050456636.Wed.Apr.16_01_30_36.GMT.2003.nordzee1.v170.170.mat D:INoordwijk 2003ไnordzee1_noQC/nst13l1050471036.Wed.Apr.16_05_30_36.GMT.2003.nordzee1.v170.170.m D:INoordwijk 2003\nordzee1_noQCVInst13\1050474635.Wed.Apr.16_06_30_35.GMT.2003.nordzee1.v170.170.ma D:INoordwijk 2003/nordzee1_noQC/nst13l1050481837.Wed.Apr.16_08_30_37.GMT.2003.nordzee1.v170.170.ma D:INoordwijk 2003\nordzee1_noQCVInst13\1050485435.Wed.Apr.16_09_30_35.GMT.2003.nordzee1.v170.170.ma

\begin{tabular}{|c|c|}
\hline & 2459 \\
\hline & 2460 \\
\hline & 2461 \\
\hline & 2462 \\
\hline & 2463 \\
\hline & 2464 \\
\hline & 2465 \\
\hline & 2466 \\
\hline & 2467 \\
\hline & 2468 \\
\hline & 2474 \\
\hline & 2478 \\
\hline & 2479 \\
\hline & 2480 \\
\hline & 2481 \\
\hline $\mathrm{CA} / \mathrm{CB}$ & 2482 \\
\hline $\mathrm{CA} / \mathrm{CB}$ & 2483 \\
\hline $\mathrm{CA} / \mathrm{CB}$ & 2484 \\
\hline $\mathrm{CA} / \mathrm{CB}$ & 2485 \\
\hline $\mathrm{CA} / \mathrm{CB}$ & 2486 \\
\hline$\overline{\mathrm{CA} / \mathrm{CB}}$ & 2487 \\
\hline $\mathrm{CA} / \mathrm{CB}$ & 2488 \\
\hline $\mathrm{CA} / \mathrm{CB}$ & 2489 \\
\hline $\mathrm{CA} / \mathrm{CB}$ & 2490 \\
\hline $\mathrm{CA} / \mathrm{CB}$ & 2491 \\
\hline $\mathrm{CA} / \mathrm{CB}$ & 2492 \\
\hline $\mathrm{CA} / \mathrm{CB}$ & 2498 \\
\hline $\mathrm{CA} / \mathrm{CB}$ & 2502 \\
\hline $\mathrm{CA} / \mathrm{CB}$ & 2503 \\
\hline $\mathrm{CA} / \mathrm{CB}$ & 2504 \\
\hline $\mathrm{CA} / \mathrm{CB}$ & 2505 \\
\hline $\mathrm{CA} / \mathrm{CB}$ & 2506 \\
\hline $\mathrm{CA} / \mathrm{CB}$ & 2507 \\
\hline $\mathrm{CA} / \mathrm{CB}$ & 2508 \\
\hline $\mathrm{CA} / \mathrm{CB}$ & 2509 \\
\hline CA & 2510 \\
\hline $\mathrm{CA}$ & 2511 \\
\hline$\overline{C A}$ & 2512 \\
\hline $\mathrm{CA}$ & 2513 \\
\hline $\mathrm{CA}$ & 2514 \\
\hline $\mathrm{CA} / \mathrm{CB}$ & 2515 \\
\hline $\mathrm{CA} / \mathrm{CB}$ & 2516 \\
\hline $\mathrm{CA} / \mathrm{CB}$ & 2522 \\
\hline $\mathrm{CA} / \mathrm{CB}$ & 2526 \\
\hline CA & 2527 \\
\hline$\overline{C A}$ & 2528 \\
\hline $\mathrm{CA}$ & 2529 \\
\hline
\end{tabular}

